\title{
NUCLEAR POWER \\ - SYSTEM SIMULATIONS \\ AND OPERATION
}

Edited by Pavel V. Tsvetkov 


\section{Nuclear Power - System Simulations and Operation}

Edited by Pavel V. Tsvetkov

\section{Published by InTech}

Janeza Trdine 9, 51000 Rijeka, Croatia

\section{Copyright @ 2011 InTech}

All chapters are Open Access articles distributed under the Creative Commons Non Commercial Share Alike Attribution 3.0 license, which permits to copy, distribute, transmit, and adapt the work in any medium, so long as the original work is properly cited. After this work has been published by InTech, authors have the right to republish it, in whole or part, in any publication of which they are the author, and to make other personal use of the work. Any republication, referencing or personal use of the work must explicitly identify the original source.

Statements and opinions expressed in the chapters are these of the individual contributors and not necessarily those of the editors or publisher. No responsibility is accepted for the accuracy of information contained in the published articles. The publisher assumes no responsibility for any damage or injury to persons or property arising out of the use of any materials, instructions, methods or ideas contained in the book.

Publishing Process Manager Petra Zobic

Technical Editor Teodora Smiljanic

Cover Designer Jan Hyrat

Image Copyright fuyu liu, 2010. Used under license from Shutterstock.com

First published July, 2011

Printed in Croatia

A free online edition of this book is available at www.intechopen.com

Additional hard copies can be obtained from orders@intechweb.org

Nuclear Power - System Simulations and Operation, Edited by Pavel V. Tsvetkov

p. $\mathrm{cm}$.

ISBN 978-953-307-506-8 


\section{INTECH INTECH open \\ free online editions of InTech Books and Journals can be found at www.intechopen.com}





\section{Contents}

Preface IX

Chapter 1 Simulation and Simulators for Nuclear Power Generation 1 Janos Sebestyen Janosy

Chapter 2 Safety Studies and General Simulations of Research Reactors Using Nuclear Codes 21

Antonella L. Costa, Patrícia A. L. Reis, Clarysson A. M. Silva, Claubia Pereira, Maria Auxiliadora F. Veloso, Bruno T. Guerra, Humberto V. Soares and Amir Z. Mesquita

Chapter 3 Development of an Appendix K Version of RELAP5-3D and Associated Deterministic-Realistic Hybrid Methodology for LOCA Licensing Analysis 43 Thomas K. S. Liang

Chapter 4 Analysis of Error Propagation Between Software Processes 69

Sizarta Sarshar

Chapter 5 Thermal-Hydraulic Analysis in Support of Plant Operation 87

Francesc Reventós

Chapter 6 A Literature Survey of Neutronics and Thermal-Hydraulics Codes for Investigating Reactor Core Parameters; Artificial Neural Networks as the VVER-1000 Core Predictor 103 Farshad Faghihi H. Khalafi and S. M. Mirvakili

Chapter 7 Recent Trends in Mathematical Modeling and Simulation of Fission Product Transport From Fuel to Primary Coolant of PWRs 123 Nasir M. Mirza, Sikander M. Mirza and Muhammad J. Iqbal

Chapter 8 Thermal-Hydraulic Simulation of Supercritical-Water-Cooled Reactors 139 Markku Hänninen and Joona Kurki 
VI Contents

Chapter 9 Non-Linear Design Evaluation of

Class 1-3 Nuclear Power Piping 153

Lingfu Zeng, Lennart G. Jansson and Lars Dahlström

Chapter 10 The Text-Mining Approach Towards

Risk Communication in Environmental Science 175

Akihide Kugo 




\section{Preface}

At the onset of the $21^{\text {st }}$ century, we are searching for reliable and sustainable energy sources that have a potential to support growing economies developing at accelerated growth rates, technology advances improving quality of life and becoming available to larger and larger populations. We have to make sure that this continuous quest for prosperity does not backfire via catastrophic irreversible climate changes, and depleted or limited resources that may challenge very existence of future generations. We are at the point in our history when we have to make sure that our growth is sustainable. New energy sources and systems must be inherently safe and environmentally benign.

The quest for robust sustainable energy supplies meeting the above constraints leads us to the nuclear power technology. Today's nuclear reactors are safe and highly efficient energy systems that offer electricity and a multitude of co-generation energy products ranging from potable water to heat for industrial applications. Although it is not inherently sustainable as solar power, nuclear technology is sustainable by design. Advanced nuclear energy systems are capable to breed new fuel, take care of nuclear waste and operate in an inherently safe way with minimized environmental effects. Catastrophic earthquake and tsunami events in Japan resulted in the nuclear accident that forced us to rethink our approach to nuclear safety, requirements and facilitated growing interests in designs, which can withstand natural disasters and avoid catastrophic consequences.

This book is one in a series of books on nuclear power published by InTech. It consists of ten chapters on system simulations and operational aspects:

- Simulation and Simulators used for Nuclear Power Generation,

- Safety Studies and General Simulations of Research Reactors Using Nuclear Codes,

- Development of an Appendix K Version of RELAP5-3D and Associated Deterministic-Realistic Hybrid Methodology for LOCA Licensing Analysis,

- Analysis of Error Rropagation Between Software Processes,

- Thermal-hydraulic Analysis in Support of Plant Operation,

- A Literature Survey of Neutronic and Thermal-Hydraulics Codes for Investigating Reactor Core Parameters; Artificial Neural Networks as the VVER1000 Core Predictor, 
- Recent Trends in Mathematical Modeling \& Simulation of Fission Product Transport from Fuel to Primary Coolant of PWRs,

- Thermal-hydraulic Simulations of Supercritical-water-cooled Reactors,

- Non-linear Design Evaluation of Class 1-3 Nuclear Power Piping,

- The Method of Text-mining Approach Towards Risk Communication in Environmental Science.

Our book does not aim at a complete coverage or a broad range. Instead, the included chapters shine light at existing challenges, solutions and approaches. Authors hope to share ideas and findings so that new ideas and directions can potentially be developed focusing on operational characteristics of nuclear power plants. The consistent thread throughout all chapters is the "system-thinking" approach synthesizing provided information and ideas.

The book targets everyone with interests in system simulations and nuclear power operational aspects as its potential readership groups - students, researchers and practitioners. The idea is to facilitate intellectual cross-fertilization between field experts and non-field experts taking advantage of methods and tools developed by both groups. The book will hopefully inspire future research and development efforts, innovation by stimulating ideas.

We hope our readers will enjoy the book and will find it both interesting and useful.

Pavel V. Tsvetkov

Department of Nuclear Engineering

Texas A\&M University

United States of America 




\title{
Simulation and Simulators for Nuclear Power Generation
}

\author{
Janos Sebestyen Janosy \\ MTA KFKI Atomic Energy Research Institute \\ Hungary
}

\section{Introduction}

This chapter deals with simulation, a very powerful tool in designing, constructing and operating nuclear power generating facilities. There are very different types of power plants, and the examples mentioned in this chapter originate from experience with water cooled and water moderated thermal reactors, based on fission of uranium-235. Nevertheless, the methodological achievements in simulation mentioned below can definitely be used not only for this particular type of nuclear power generating reactor.

Simulation means: investigation of processes in the time domain. We can calculate the characteristics and properties of different systems, e.g. we can design a bridge over a river, but if we calculate how it would respond to a thunderstorm with high winds, its movement can or can not evolve after a certain time into destructive oscillation - this type of calculations are called simulation.

For simple systems we probably can reach an analytical solution to show that a given system is damped enough to stay stable without oscillation even in very different circumstances. Simulation steps in when the systems are too sophisticated to reach any analytical solution. Unfortunately, if we want to reach correct and accurate results we usually end up with very sophisticated and non-linear system description. This unavoidable leads us to simulation.

According to some authors, probably the last engineering achievement made completely without simulation was the Empire State Building. The Boeing 777 was mentioned as the first construction the design of which was completely unthinkable without simulation. (Janosy, 2003)

We need simulation if:

- The processes are too sophisticated and they have too many physical states just to think about everything

- It is too expensive and/or dangerous to build a prototype just for testing - or even if we have a prototype, we are very limited in testing and checking it under very different circumstances due to the costs and unavoidable dangers

- We want to check properties and compare different solutions under extreme conditions. All these conditions are present in designing, constructing or operating a nuclear power generating system (Janosy, 2007 November). 
The process of simulation can be accomplished with or without human interaction. Earlier the common way of doing it was to write a simulation program, to prepare input data sets, run the program on a powerful computer system and wait for the results. Most of the analyses of accident scenarios are being done this way even nowadays.

We already know for long time that we can save significant time and effort if we can participate in the process of simulation. We should watch the results from the very beginning, and we should have means to interact with the process, to change inputs and influence this way the sequence of the events. If our computer is capable to do that, then we have a simulator.

\section{Modeling and simulation}

It is easy to understand that no simulation can be done without prior modeling. Modeling nowadays means exceptionally mathematical modeling. We have to study the processes in question, and try to find the proper formalism to describe them correctly with mathematical expressions and tools.

Even nowadays, in the era of cheap and abundant computational power it is essential to differentiate between dominant and unimportant processes. Even if we can afford extremely fast computers, not eliminating the unimportant processes and modeling everything we can think of, leads to enormous problems during verification and validation of our models.

\subsection{Types of mathematical models}

Continuous processes can be described by set of differential equations. If only the time dependence is important, we construct a set of ODEs (Ordinary Differential Equations), where all derivatives are taken only by time. Sometimes these models are called as 'point models' because they have no space dependence; they depend only upon the time. If all the derivatives can be described by separate functions, we get the following (rather simple) form:

$$
\begin{array}{rlrl}
\frac{d y_{i}}{d t} & =f_{i}\left(y_{1}, y_{2}, \ldots y_{n}, p_{1}, p_{2}, \ldots p_{k}, t\right) ; & & i=1, \ldots n \\
z_{j} & =g_{j}\left(y_{1}, y_{2}, \ldots y_{n}, p_{1}, p_{2}, \ldots p_{k}, t\right) ; & j=1, \ldots l
\end{array}
$$

where $y$ : the state variables; $p$ : the input; $z$ : the output variables

Sometimes these functions $f$ and $g$ cannot be separated so nicely and easily, sometimes we have to iterate, etc. Nevertheless, practically all numerical solution methods need to get the values of all derivatives explicitly.

If we have to take into account the space dependence as well, we get a set of PDEs, (Partial Differential Equations). Presuming again that we can define separate functions for each derivative, we get:

$$
\begin{array}{rlrl}
\frac{\partial y_{i}}{\partial x_{j}} & =f_{i, j}\left(y_{1}, y_{2}, \ldots y_{n}, x_{1}, x_{2}, \ldots x_{k}, p_{1}, p_{2}, \ldots p_{m}, t\right) ; & & i=1, \ldots n, j=1, \ldots k \\
\frac{\partial y_{i}}{\partial t} & =g_{i}\left(y_{1}, y_{2}, \ldots y_{n}, x_{1}, x_{2}, \ldots x_{k}, p_{1}, p_{2}, \ldots p_{m}, t\right) ; & & i=1, \ldots n \\
z_{j}=g_{j}\left(y_{1}, y_{2}, \ldots y_{n}, x_{1}, x_{2}, \ldots x_{k}, p_{1}, p_{2}, \ldots p_{m}, t\right) ; & j=1, \ldots l
\end{array}
$$

where $y$ : the state variables; $p$ : the input; $z$ : the output variables; $x$ : the space coordinates. 


\subsection{Discretisation in time and space}

If we want to solve our equations numerically, we have to discretise them by time and space. Discretisation in time means that instead of the continuous solution for each state variable and each output we get time series, e.g. discrete values valid only at given time instances. The time difference between two consecutive time values is called as 'time step of integration'. Instead of time derivative the differences of the consecutive values of the state variables are used, divided by the time step.

The same is true for space discretisation, frequently called as nodalisation. Instead of continuous functions we get discrete time series of state variables for each node, having finite volume and finite distance between them. (The same way, instead of the space derivatives this finite distance is used in the equations to divide the difference of the state variables in two neighboring nodes.)

The stability and accuracy of the numerical solution highly depends upon the time step of the integration and of the space distance of the nodalisation. It is quite obvious that the smaller is the time step, the smaller are the nodalisation distances and the sizes of the nodes, the better is the stability and accuracy of the solution. On the other hand, making the time step and the nodalisation grid smaller increases the number of the state variables and the necessary computer power.

Sometimes physical processes happening at the same time and space are divided and solved separately. Usually the neutron-physical processes of heat generation and thermo-hydraulic processes of the heat removal are solved by two separate programs. The first calculates the heat to be removed from the core of the reactor, the second the temperatures of coolant and fuel as result of the cooling process. The time step of the data exchange between these two simulation programs should be small enough not to generate remarkable errors as a consequence of this separation.

There are advanced mathematical methods to solve a system of differential equations. Remarkable computer resources can be spared using so called multistep methods, that means the next value of a variable is calculated not only using the previous one, but a sequence of previous values. Unfortunately these multistep methods cannot be used if discrete events happen between two acts of solution (e.g. rod drop or valve closure). These events are causing discontinuities in the high-order derivatives, which are usually not allowed if using multistep methods.

Logical functions and event sequences usually are not simulated by differential equations, but by separate programs dedicated to this purpose. Protections, interlocks and other similar functions of the process instrumentation are modeled this way.

\subsection{Model verification and validation}

After the modeling has been finished and before any simulation is started, we have to verify and validate our simulation system.

In our case verification means, that our model and the numerical solution system is working according our intentions. The model equations are correct and free from programming errors, and the same is true for the numerical solving programs. The solution is stable and accurate.

This can be verified using so called benchmark tests. These are well-known experimental results, measured on different experimental facilities. They are usually much smaller than a nuclear power generating unit, but specially tailored to demonstrate sophisticated physical phenomena which are not allowed to test on a real plant - e.g. pipe break causing the 
coolant to flow out. Usually we have a two-phase outflow (steam and water) coming with the speed of the sound.

Sometimes it is not necessary to develop sophisticated model programs with elaborated numerical solving schemas. For example, to simulate thermo-hydraulic processes inside the primary circuit of a pressurized water reactor (PWR) we can use the RELAP program (developed in the USA), the ATHLET code (developed in Germany) or the CATHARE code (developed in France). Several millions of dollars and hundreds of man-years have been spent to develop and validate them, against great many experiments.

Even using well-validated and certified codes we cannot omit the validation process. During validation we have to show that the input data made for these codes correctly describes the nuclear power generating unit in question. The nodalisation corresponds to the actual geometry of our plant and it is prepared according to the rules prescribed in the user manual to the given code. All masses, heat exchange surfaces, heat capacities, heat conductance etc. are calculated for each node correctly. Some simple transients which happens sometimes on the plant and are not regarded as accident (e.g. pump trips, turbine trips, network frequency control acts etc., usually called as AOO - anticipated operational occurrences) are calculated with the code and compared with the measurements in order to show, that the current and parameterized for the given plant model is valid.

\section{Classification of simulators}

The simulation of the desired process can go faster or slower than the real time, even both can happen during one act of simulation. The beginning of an accident or even a transient may require more computational power than the (asymptotic) end of it. If we do not care the relation between the simulated time and the real time too much, then we have an engineering simulator. The only thing what differs it from an off-line simulation program is the interactivity provided by the man-machine interface to the simulation code.

Sometimes it may happen that we want to test a ready-made controller hardware before putting it into operation on the real plant, or we want to teach people how different scenarios should be handled in the control room. In this case we should have a simulator which always runs in real time. (Controllers or people cannot tolerate a simulator running faster or slower than the real process.) This way we get a development simulator or training simulator.

The best way to teach people is to have a replica control room for interaction, and therefore a replica simulator. Only these simulators can provide the so called "hand-on" training, showing an environment very similar to that on the real nuclear power plant. Moreover, if the operations are not limited only to some panels in this control room but all switches, meters and annunciators of the real control room are handed correctly by the simulator, then we have the king of all simulators - the full-scope replica training simulator.

The simulation time step of the modern training simulators is around $0.1 \ldots 0.2$ seconds. For the processes shown in the control room practically we do not need smaller ones. Of course, there are some processes faster than that but usually they cannot be presented to the operators in the real control room, either. Several decades ago because of the slower computers the time step was chosen around 1 second, and this was not too bad, either.

However, the man-machine interface between the simulator and the operator is a different question. If an analogue meter moves only once per second it is very unrealistic and disturbing for the operator. The same is true for the actuator. Pushbuttons sensed only once per second are not very realistic but control actions performed in the control room are even 
worse. It is impossible to set a valve or control rod to an exact position if the time resolution of these inputs is only one second or even 0.2 second. It can happen that the new valve position is calculated only once per second but the time of action (how long a pushbutton has been pushed) must be measured and presented to the model programs with much greater accuracy.

Therefore the scanning frequency of the control room must be not slower than once per $50 \mathrm{msec}$ or once per $100 \mathrm{msec}$. Analogue values shown on the meters should be interpolated with this frequency and each operation in the control room - pushing or releasing switches, etc. - should be accomplished with a time stamp of this resolution.

\subsection{The absolute necessity of the training simulators}

It is quite understandable, that we need simulator training if the device to be learned

- $\quad$ is very expensive to build and operate

- $\quad$ can lead to dangerous and even lethal consequences if operated erroneously.

Everybody understands easily that e.g. jet pilots should be trained on simulators. In case of nuclear power we have two more reasons to do so:

- Jet pilots are taking off and landing daily. Probably they can maintain their knowledge having this kind of practice. On the other hand, nuclear power plants are started and shut down for re-fueling normally once per year. During the whole year practically they are operating on the maximal possible power. The knowledge and the preparedness of the operators to deal with any situation may be kept on necessary level only with regular simulator training.

- Since the Chernobyl accident emerged from a not-properly-designed-and-executed experiment, it is practically impossible to get authorization to make experiments on an existing nuclear power plant. New ideas, new control and protection systems, new types of technological units are required to be tested thoroughly on development simulators. If they have man-machine interface consequences, then the required simulators should be replica - even better: full-scope replica simulators.

These considerations increase significantly the importance of simulators in the nuclear power generating industries.

\subsection{Simulation in design and authorization}

Nowadays it is more difficult to get the approval of the authorities for constructing a new nuclear power plant than to accomplish the construction itself. The design and the authorization are "handshaking" processes with many stages. Not going into detail, during these processes the so called Safety Report has to be worked out, too. Part of this report deals with different possible scenarios. Some definitions of basic importance:

The design basis accident is defined as follows:

A postulated accident that a nuclear facility must be designed and built to withstand without loss to the systems, structures, and components necessary to ensure public health and safety.

The beyond design basis accident is defined as follows:

This term is used as a technical way to discuss accident sequences that are possible but were not fully considered in the design process because they were judged to be too unlikely. (In that sense, they are considered beyond the scope of design basis accident that a nuclear facility must be designed and built to withstand.) As the regulatory process strives to be as 
thorough as possible, "beyond design-basis" accident sequences are analyzed to fully understand the capability of a design.

Naturally, all these accidents, transients and scenarios are evaluated and studied by means of simulation programs. These programs are being developed by few nations only (USA, Russia, Germany, and France) because the development and the verification is a rather expensive and lengthy process: not every country can afford it and on the other hand, it is not necessary to do so, too. These programs are usually developed for a given reactor type and can be used for a certain family of nuclear power plants. Usually there are no restrictions in participating in the development and in the usage of these simulation programs. It is done on the basis of bilateral agreements.

Practically there are three phases of the usage of these programs:

- Development of the simulation package itself, verification and validation using different benchmark test results

- Model construction for the simulation package, using design data of the nuclear power plant in question; verification and validation of the constructed models using data available from similar nuclear power plants

- Generating different accident and transient scenarios for the safety report using the "worst

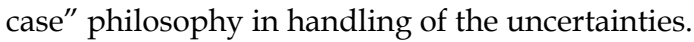

\section{Simulators for training and development}

It is common for these simulators that - dealing with people and real equipment under test

- $\quad$ they should be running in real time.

\subsection{Model programs and data storage requirements}

First it has to be defined, what are the basic requirements to the model programs and the data storage facilities of the training simulator.

The simulator programs are started together with the whole training simulator. During the initialization phase it is allowed to set up different data tables etc., even reading files. After initialization the programs of the mathematical models are waiting for the command of the main control program of the simulator, the real-time executive.

All state variables defining the current state of the model - and therefore the state of the simulated power plant - should be located in the real-time data base (see Fig. 1). This 'data base' is usually just a manageable piece of a shared memory. The exact (binary) copy of this piece of memory can be used as a fully defined snapshot of the state.

After getting the proper command from the real-time executable the model programs advance one time step and based on the previous state (the results of the previous step) they calculate the state of the plant in the next step. Meanwhile, the actions of the operators in the Control room are scanned by the man-machine interface (MMI programs) asynchronously with a much shorter time step. The actions are stored in the I/O data base and are used by the model programs.

If the time step is short enough (e.g. $0.2 \mathrm{sec}$ or less) then the model programs can use only one copy of the state variables. Before the actual step it contains data belonging to the last step. During the execution of the model programs the state variables are calculated for the next step one by one, and after the execution they all belong to the next step. Frankly speaking, it is not exactly correct that some model programs should use data form the 
previous and the next step simultaneously, but if the time step is small enough - and it is then this fact cannot cause big errors.

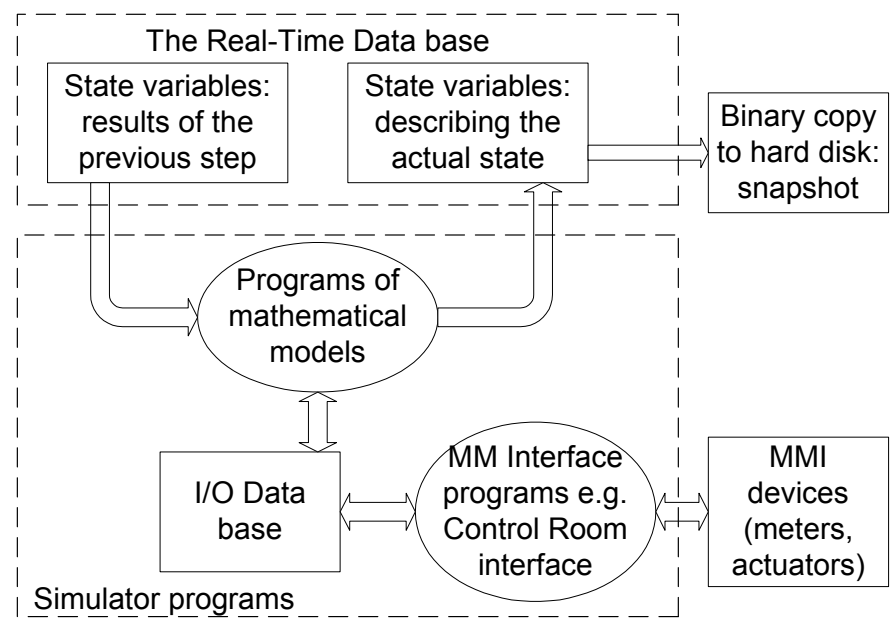

Fig. 1. Simulator programs and data storage

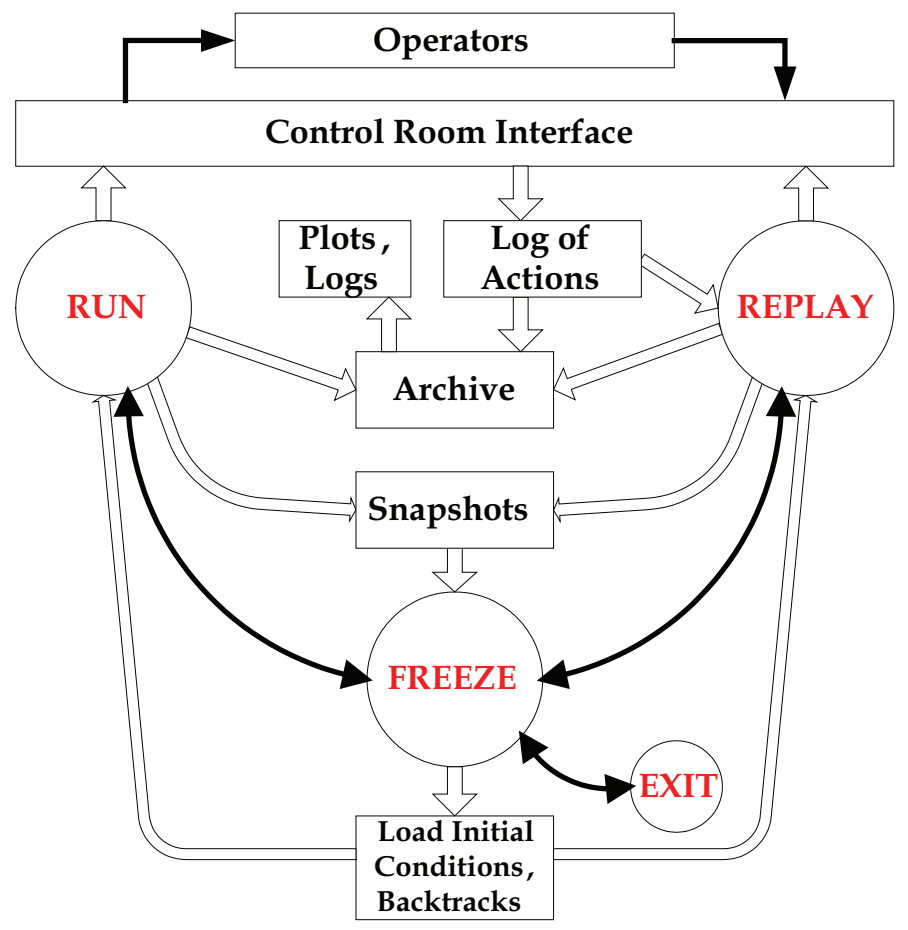

Fig. 2. States of the training simulator 
If we decide to use this approach - having only one copy of state variables in the memory; it is quite common in simulators - then we have to consider not to allow access to these state variables until the actual act of integration is not finished. Accessing the state variables any time means that other programs may fetch values not belonging to the same time instance. For analogue values it is not a big problem, but solving logical circuitry it can lead to confusion and incorrectness.

The different states of the simulator are presented on Fig. 2. Black arrows are state transitions, white arrows symbolize data flow.

After starting up the simulator and all related programs (from the EXIT state) we reach the FREEZE state. All programs are able to run, but practically neither of them is actually running.

During the FREEZE state we are able to initialize the simulator, either loading in a saved Initial Condition, or a previously saved snapshot (loading it is often called backtracking).

Adjusting switches in the Control room to the actual loaded state may become necessary; it is done using the Control room set-up report made by the CR I/O system.

From the FREEZE state we can move to the RUN state.

The model programs calculate cyclically the actual state every time step and all the analogue meters and annunciators of the Control room are driven accordingly. All the operations of the staff are scanned in and stored in the Log of actions, and are added to the Archive, together with the history of the most important parameters - there are several hundreds of them. After the simulation session is finished, different logs and plots can be generated for evaluation of the trainees.

During the simulation snapshots are taken regularly, or at any instant if the Instructor of the actual simulation session commands to do so. Any time the instructor can stop the simulation and return to the FREEZE state. During FREEZE state the parameters are displayed in the Control room, and the situation can be analyzed. No operations can be performed, though.

From the FREEZE state we can re-play how the operations happened earlier in the control room. Backtracking using a snapshot, made earlier, we can enter the REPLAY state. During the REPLAY state all the simulation is performed and the control room is driven as during the RUN state, with the exception that no actions are accepted from the operators. All operations are taken from the Log of actions, with their time stamps together, therefore the trainees are able to follow what and how it happened earlier in the Control room.

Any time the REPLAY can be stopped, the state turns to the FREEZE state, and real operations can commence entering the RUN state again. This is a very useful ability for the Instructor, to go back in time, to show when and how a mistake was done, what are the consequences and how it should be continued in a correct way.

\subsection{Architecture of the simulators}

Practically the full-scope replica simulator consists of the following parts:

- Computer system of the simulator. It incorporates the model programs, the simulation control programs, the loggers, plotters, the archive etc. necessary to conduct a simulation session.

- Control room and interface devices - a replica of the real control room equipped with all meters, switches, pushbuttons, screens, memo-schema etc. and the hardware/software devices (the MMI, the man-machine interface) enabling the computer system of the simulator to handle them quickly and correctly. 
- Instrumentation and control devices taken from the real power plant. It is very advantageous if we can take over the plant computer system, the core surveillance system and other systems directly from the plant.

- The Instructor's system, the basic tool of the Instructors to control the simulation session. The Instructor's system is usually hidden from the trainees, but sometimes, when the Instructor is present in the Control room, he/she can use a remote control unit to activate different pre-programmed events.

It is obvious that it is much better to use the real plant computer, the real core surveillance system instead of simulating them. The operators feel the real controls; the real functions of these units can be studied. The problem lies in the simulator functions to which these real instruments are poorly suited. No real plant computer etc. is prepared to the stopped and standing time, or even worse: to the backtracking, going back in time. It is difficult to accommodate the real equipment to the new initial condition loaded into the simulator (e.g. nominal state immediately after the cold shutdown state). It is obvious that all functions somehow connected to the time (logging, making archives, and plotting) should be excluded if possible. If they cannot be excluded: we have to refuse to integrate the real units, we have to model their functions.

That is the main reason that all time-related functions are incorporated to the Instructor's system: logging, plotting, making archives etc. etc. On the other hand, all simulator-specific functions are evaluated in the simulator.

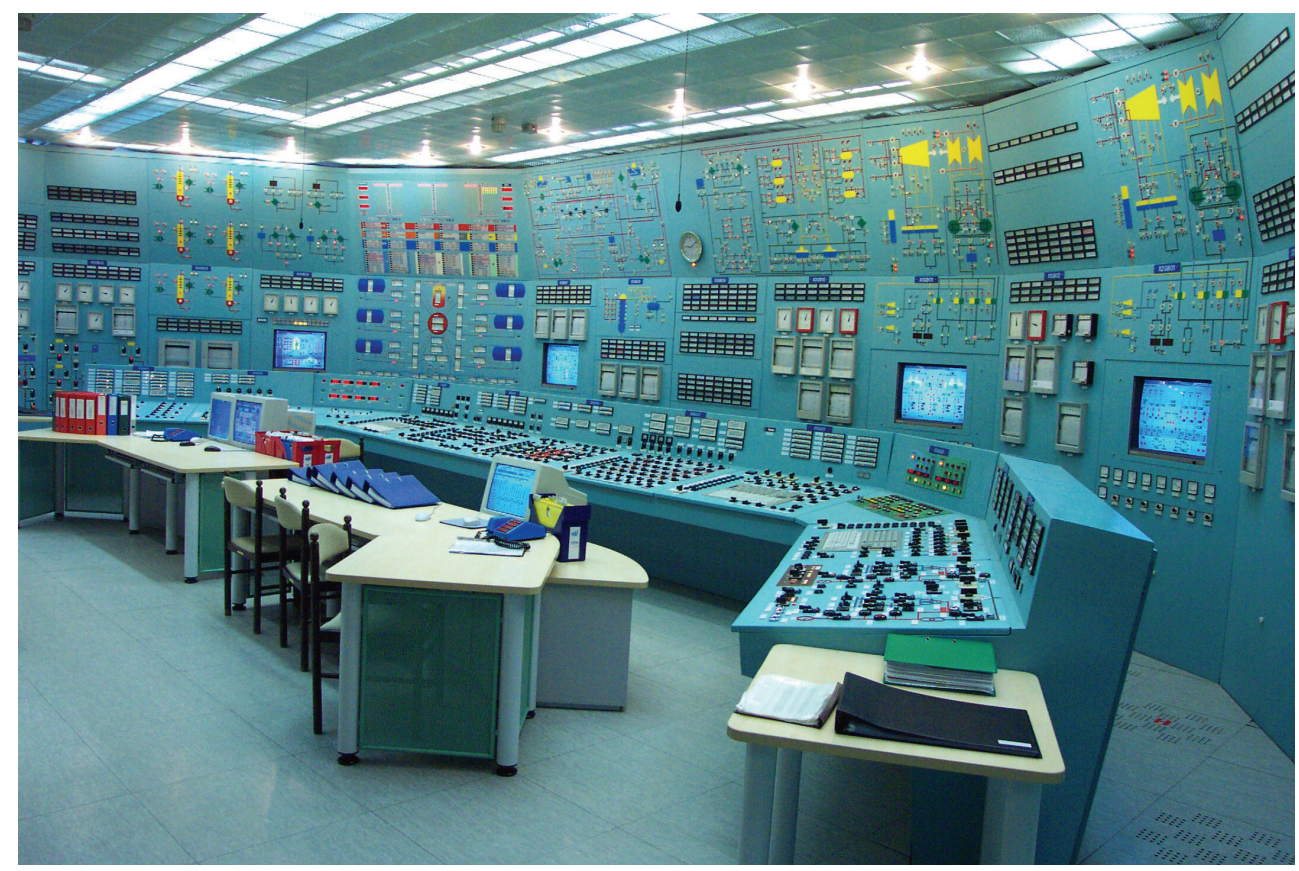

Fig. 3. The replica control room of the Paks NPPs training simulator

The most important function is the pre-programming of the malfunctions. All valves can leak, all pipes can break, all pumps can be tripped, and there are very many equipment- 
specific malfunctions. They can be activated promptly, or at a given time instance, and/or when a logical function becomes 'true' (e.g. IF the temperature is higher than ... AND the flow is less than ... etc.)

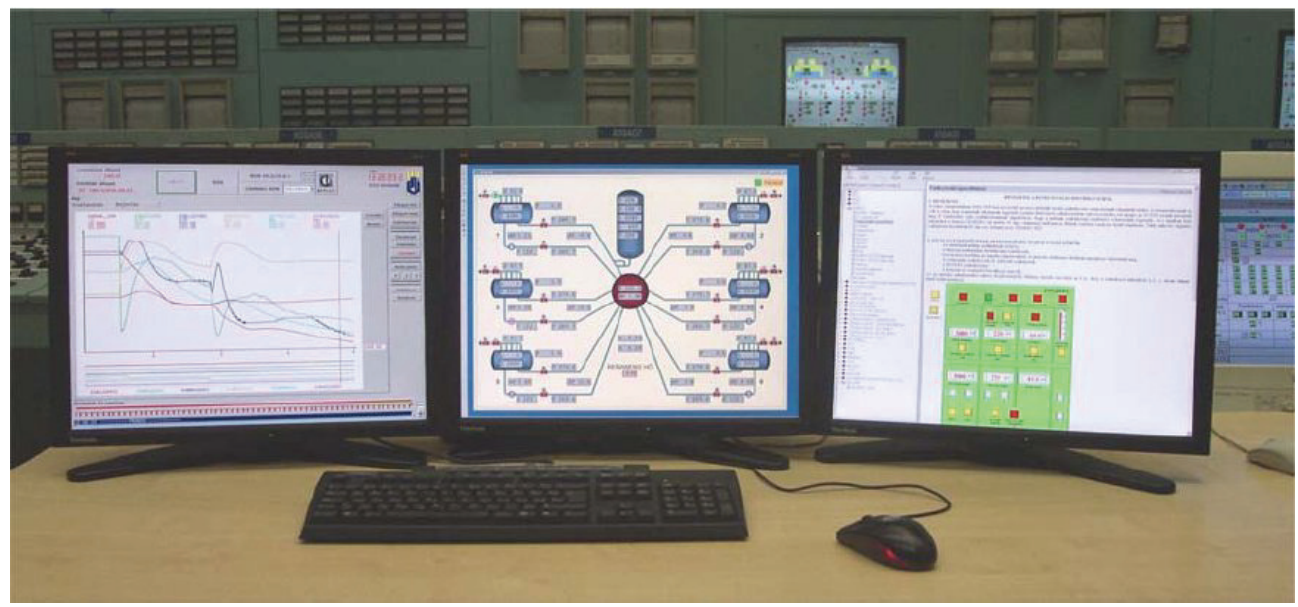

Fig. 4. The instructor's workstation of the Paks NPPs training simulator

\subsection{Protection system refurbishment using simulator}

The existing nuclear power plants were licensed earlier usually for 30 years; most of these licenses expire in the next decade. Nowadays it is a common practice to prolong the operation of the NPPs up to 50-60 years.

After the Chernobyl accident in 1986 requirements to the safety of nuclear power generation units has been changed dramatically. As a result, many enhancements have been introduced not only to the Instrumentation \& Control (I\&C) and Protections circuitry but to the technological systems as well. Practically all these changes have been introduced on the simulators first, in order to show the results of the forthcoming changes.

Even without that the "moral" and practical lifetime of the I\&C systems is much less than 50-60 years, let say only 8-10 years. If they contain computers (and nowadays they do) this becomes even shorter, about 5-7 years. The "aging" IT systems cannot be kept running for a longer time. Spare parts and even software drivers become obsolete.

Replacing protection and control systems is relatively easy if the functionality remains the same. Fig. 5. shows how it can be done.

First, while the old system is still in charge, the new system is placed parallel to it. Both controllers (or others, as protections, interlocks) get the same inputs. The new controller should be tuned until the response becomes the same in rather different situations, too. Then the old controller can be replaced. This method cannot be used when it is dangerous or just it is not allowed to test the equipment in extreme conditions. It is a rather new practice to use simulators for I\&C or other system's refurbishments (Janosy, 2007 March). First the simulators are used during the design of the new systems (Janosy, 2008). Integrating software models of the newly designed models into the simulator in an interchangeable way the proposed functionalities can be tested in normal, accidental and even extreme circumstances (software-in-the-loop tests). After approval of the demonstrated functions 


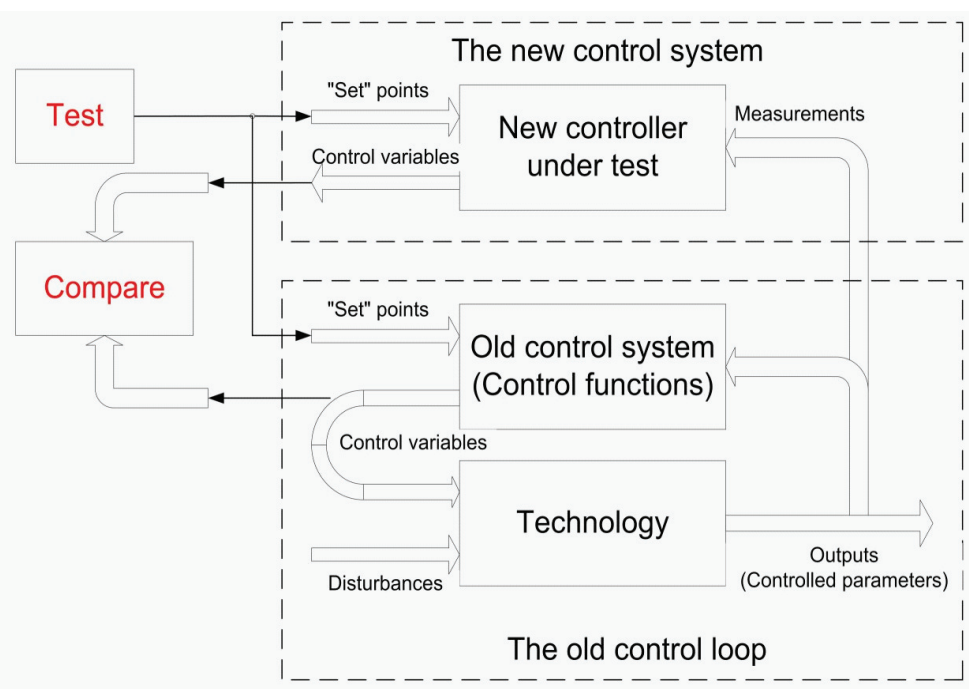

Fig. 5. Old and new controllers tested in parallel

and performance, the manufacturing of the new hardware can be authorized. The new hardware should be attached to the simulator, too, and the functionalities and the performance can be compared with its already existing software model (hardware-in-theloop tests).

As it was mentioned before, it is not very easy to integrate real I\&C hardware to the simulator because of the special simulator functions of FREEZE, BACKTRACK, REPLAY. This procedure had to be organized as it can be seen on Fig. 6 .

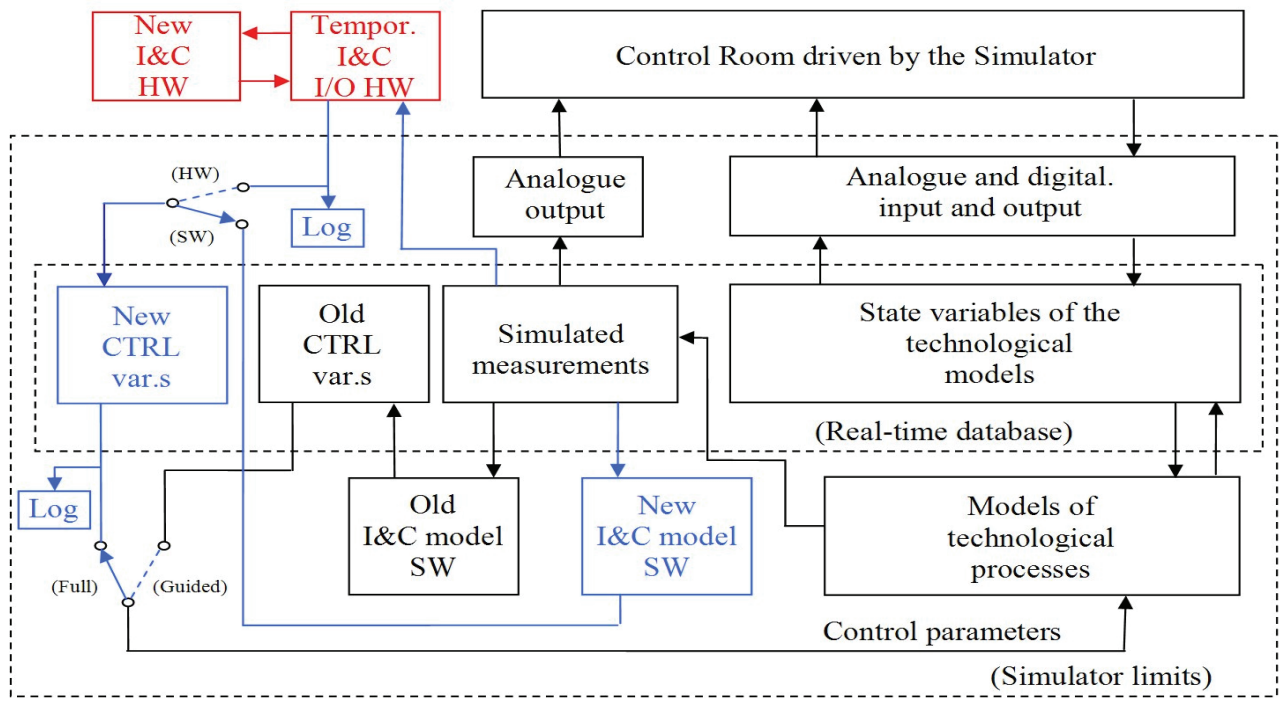

Fig. 6. Instrumentation and control system $(\mathrm{I} \& \mathrm{C})$ is tested on the training simulator 
The black color indicates the original functions of the simulator. The technological models advance in time using their state variables. The value of the measurements are calculated and the old I\&C models calculate the control parameters (e.g. control valve and rod positions) governing the technological models. The development of the new system is made in four consecutive steps.

1. The new controllers' mathematical models are constructed and their simulation models are placed parallel with the old one (blue boxes). On the basis of the same measurements the new model calculates the control parameters. In this phase the (software) switch is placed to the (Guided) position, that means that the control actions of the new controller are only logged, the old controller model is in charge.

2. If everything looks perfect, the switch is thrown into (Full) position, and the 'software in the loop' mode is achieved.

3. After thorough testing the new controller is manufactured and using some temporary $\mathrm{I} / \mathrm{O}$ hardware interface (red boxes) it is connected to the simulator. (Spare parts of the Control room I/O can be used). The new hardware is driven by the measurements, too, but the new software governs the simulator - (SW) and (Full) position of the switches.

4. If according to the logged response of the hardware is $\mathrm{OK}$, the upper switch can be thrown to (HW) position. This is the 'hardware in the loop' mode of operation.

Thanks to the simulator, the new I\&C equipment can be tested under extreme conditions, too, without the slightest economic and environmental risks.

Practically everything can be tested before the plant stops. During the refueling - which usually takes more than 20 but less than 30 days - the new equipment can be integrated to the real unit and in the same time the idling operators can study the behavior of the new I\&C on the simulator in 'software in the loop' mode.

\section{Nodalisation problems of the reactor models}

The most important and difficult part of the simulation programs and the simulators is the reactor model. Fuel elements, integrated into fuel assemblies produce heat in the nuclear reactors in rather difficult, harsh conditions. The pressure and temperature is high - up to $160 \mathrm{bar}$ and $320^{\circ} \mathrm{C}$ - and the power density in some reactors reaches $90 \mathrm{~kW} /$ liter and above. They are made from expensive metals using expensive technologies. They should not leak the cladding represents the first barrier between the radio-active materials and the environment (usually there are at least three barriers). If there is a remarkable leak, the reactor should be stopped and the leaking fuel assembly replaced - a procedure causing significant economic loss.

Nevertheless, some fuel assemblies are well made and they practically never leak. During the 20-year-history of the four-unit Paks NPP there was detectable leak only once or twice. The fuel elements originally spent three years in the core, nowadays they stay for four years - with slightly higher uranium content, of course. If they should stay for five years, the increasing of the enrichment is not enough - the control system of the reactor is not designed to cover the excessive reactivity of the core, produced by the higher enrichment of the fresh fuel.

The solution is the Gadolinium (Gd) which is a burnable neutron poison. In the first year or so - it helps to cover the excessive reactivity by absorption of neutrons, then it burns out and do not causes any problem in the upcoming years. Now we replace at the Paks NPP every year $1 / 4$ th of the fuel elements with fresh ones. If we start to replace them with the 
new types, supposed to stay for five years, it means that we are going to use mixed cores at least for four years. These cores need special treatment and the operators should be trained to it. The core surveillance system must be fitted to these mixed cores, too. To train the operators to their more sophisticated duties we had to replace the reactor model and the model of the primary circuit with more elaborated 3D spatial models.

We have 349 fuel assemblies in the core; each of them can be of different age and different composition. The core configuration is carefully optimized each year to ensure that the power distribution and burn-out corresponds to the maximal safety and to the best fuel economy. Careful design of the reactor loads results in negative temperature and volumetric coefficients that means that the reactor is capable to self-regulate its power - because making the coolant hotter and thinner means worse neutron balance and therefore it decreases nuclear power.

These effects make the neutron kinetic model of the reactor and the thermo-hydraulic model of the primary cooling circuit tightly coupled; therefore they mathematical models must be solved simultaneously. Describing very different physical phenomena we get very different equations - that leads to severe problems of the simultaneous numerical solution. (Hazi, Kereszturi et al., 2002)

The crucial point is: how to nodalise the nuclear reactor and the primary circuit in order to achieve high fidelity of simulation with reasonable computer loads - in other words achieving accurate simulation and still remaining in real-time. It looks easy to divide the equipment to very small parts, and solve the problem using them as coupled nodes. Decreasing the size of the individual nodes not only increases their number according to the third power, but in the same time it significantly decreases the necessary time step of the numerical integration.

\subsection{Nodalisation problems: Neutronics}

As it is shown on Fig. 7, we have in the core 349 hexagonal fuel assemblies (the numbers outside the core refer to the six cooling loops). The 37 numbered fuel assemblies are used to control the chain reaction. They are twice as long as a normal fuel assembly. The upper part is made from special steel designed do absorb the proper amount of neutrons in order to be able to control the chain reaction. The lower part is a usual fuel assembly containing usual amount of fuel. Pulling out this control assembly means that the lower part enters the core, lowering it causes this part to leave and to be replaced by the neutron absorber assembly.

The 37 control assemblies are organized into 6 groups, containing 6 assemblies except the 6th one, which contains 7 (this 7 th is the central one). The first five groups with 30 assemblies are used as the "safety rods", fully pulled out during normal operation and fully lowered during reactor shut-down. The 6th group is normally used as "control rods", during normal operation they are always in different intermediate positions according to the prescribed power of the reactor. In some very rare situations the 5th group is helping to the 6th one, sometimes staying in intermediate position, too.

That evidently means that the first four groups do not influence the spatial distribution of the neutrons, their absorbents are pulled out and their fuel assemblies are inserted. Lowering them the reactor is shut down and the spatial distribution is not interesting any more. In the same time, the last two groups - the 5th and the 6th - can seriously influence the $3 \mathrm{D}$ distribution of the neutrons, being in different intermediate positions according to the different operating conditions of the reactor and the primary circuit. 
The nodalisation of the core from the neutron kinetics point of view does not leave us too much freedom: each "neighbor" to each assembly can be of different "age" in the reactor (zero to four, later zero to five years), with or without Gadolinium content accordingly. Different "age" means different burn up, thus different stage of enrichment and different isotope content. That means that in horizontal plane each assembly should be a separate node. As to the vertical nodalisation, we must have not less than 8 or 10 planes to get enough resolution (8 to 10 points) to describe the axial neutron (and heat) distribution. We have chosen 10 planes vertically - that means, we have finally $349 \times 10$ nodes for the KIKO3D model (Kereszturi et al., 2003).

Real-time spatial (3D) simulation of 3490 nodes in several groups of neutrons according to their actual energy requires huge computer power. The only way to do it using several processors; it means to separate the time and space problem. The result can be written as a product of two functions: the amplitude function of time and the distribution function of space. Solving the equations in different processors means that these programs have access to the data of the other only after finishing the actual time step, and this means that delays are introduced.

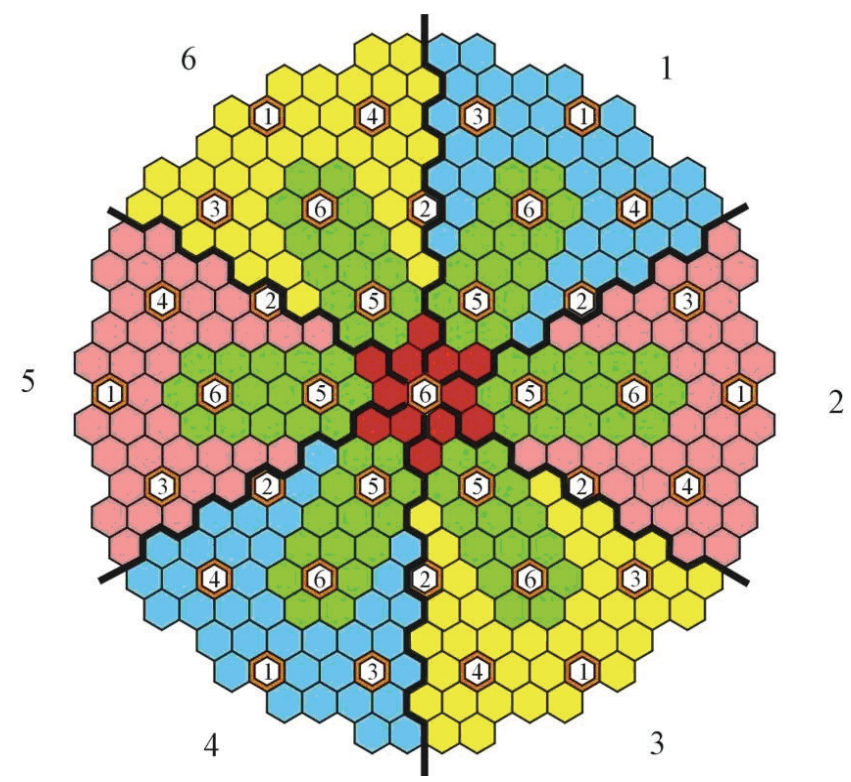

Fig. 7. The map of the core with the 349 fuel assemblies, including the 37 control ones

\subsection{Nodalisation problems: Thermo-hydraulics}

Thermo-hydraulic nodes should be much larger in space than the neutron-kinetic nodes. It is connected with the $0.2 \mathrm{sec}$. time step of the full scope replica simulator of the power plant. If we want to avoid large number of iterations, the amount of the steam/water leaving/entering the node each time step must be probably less than the full amount of the steam/water inside the node. It means that if we multiply the maximal feasible volumetric flow-rates with the $0.2 \mathrm{sec}$. integration time step, we get the minimal volumes for the nodes in question. 
Fig. 7. shows the thermo-hydraulic nodalisation of the reactor as well. According to the reasons explained above, we have much less thermo-hydraulic nodes radially in the core, and the number of the axial layers is only half that of the number of neutronic nodes: we are limited here to only five layers. The reactor core is divided radially to only six outer, six inner and one central node: altogether 13 nodes.

Each of the outer six nodes marked with different colors consists of 40 fuel assemblies. The inner six nodes - all are marked as green - contain 16 fuel assemblies each but one of them belongs to the $5^{\text {th }}$ control rod group, one of the to the $6^{\text {th }}$ control rod group. The $13^{\text {th }}$, the innermost small node contains 13 fuel assemblies, one of them - the central - contains the $7^{\text {th }}$ rod of the $6^{\text {th }}$ group.
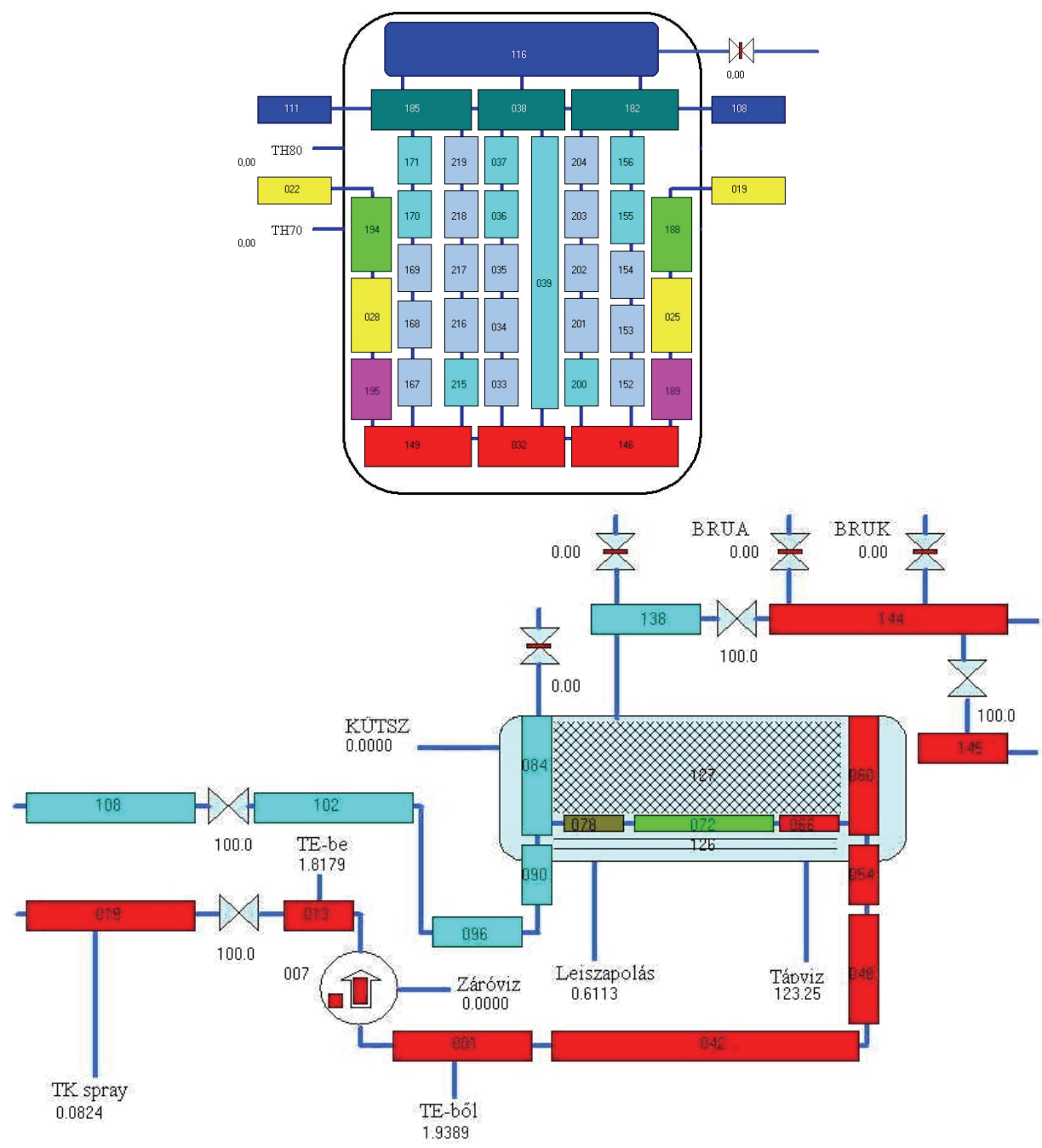

Fig. 8. Debugging tool for the core nodalisation and for the six cooling loops 
This kind of thermo-hydraulic nodalisation provides the following benefits:

- During operation on power, only control rods of the $5^{\text {th }}$ and $6^{\text {th }}$ control rod group may have intermediate positions, influencing the spatial distribution of the neutrons. The inner 6 nodes and the central node are responsible for the calculation of these effects.

- One or more cooling loops may fail, usually because of the tripped main circulating pumps (MCPs). The six outer large nodes can respond spatially to these effects.

The thermo-hydraulic model has to deal not only with the reactor vessel but the six cooling loops of the primary circuit.

The original instrumentation of the nuclear power plant can not show the power, the pressure, the temperature and the steam content of the water in each simulation node of our simulator. It is not necessary to measure these parameters in such detail for the operation of the plant. It means that the full-scope replica simulator has no tools to follow the actual values in these nodes. For development and debugging we had to develop special debugging tools (programs) to display these data on different windows on the screen in order to follow the performance of our model programs closely (see Fig. 8).

Thanks to the nodalisation scheme described above, different spatial effects in the core can be studied. As an example, the "rod drop" malfunction is presented.

If a control rod erroneously drops into the core, the negative reactivity caused by it can be compensated by the power controller, pulling all the other rods a little out from the core. However, the power locally will be less around the fallen neutron absorber.

All well-designed reactors are self-regulating, that means overheating causes negative reactivity thus decreases the heat power, and overcooling does the opposite - it leads to positive reactivity and the power increases a little. This effect compensates the locally introduced (by the fallen rod) negative reactivity, and that's why the distortion of the power field - and the resulting temperature field - is not so strong than it could have been expected, not taking into account the temperature effects and the resulting self-regulating features.

However, the resulting asymmetry of the temperature field is remarkable, as it can be seen on Fig. 9.

\subsection{Reactor thermo-hydraulics}

Normally the primary circuit of a pressurized water reactor is filled up with water and there is no boiling. Nevertheless, we have the pressurizer with the steam cushion, the secondary parts of the steam generators with boiling, the steam headers, therefore we have to construct a two-phase-flow (steam, water) model anyway.

Moreover, in case of LOCA (Loss of Coolant Accident) if the water flows out through the break of some pipelines, the emergency core cooling circuits step in, pump water into the primary circuit and the reactor vessel in order to keep the core covered with water and cooled. Air and other non-condensable gases may enter the primary circuit. During the startup of the plant the initial pressure is reached by nitrogen cushion in the pressurizer. Because these states the simulation model should handle not only water and steam, but noncondensable gases (third component.)

The best tool to simulate these states is the so called " 6 equation" model - energy, mass and momentum balance equations for steam and water separately, but with enabled state changes (boiling, condensation). (The non-condensable gases usually are added to the steam phase but state changes are disabled for them).

Solving 6-equation models in real time is an exceptionally demanding task, requiring very powerful computers. Things are getting much simpler using 5 equation models (common 


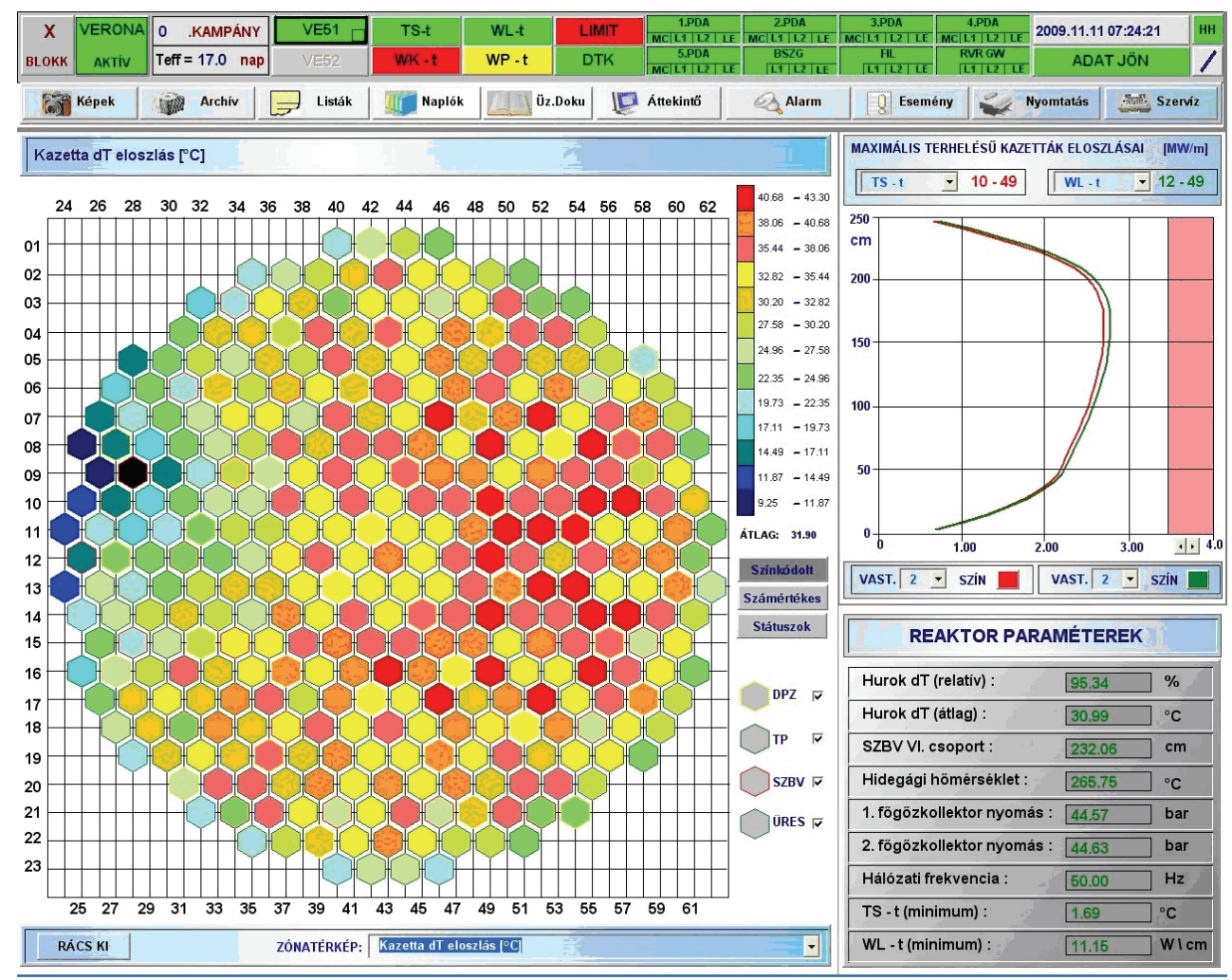

Fig. 9. Picture of the in-core surveillance system VERONA - driven by "rod drop" state data from the simulator

equation for the momentum of steam and water) but in this case the water and steam velocity should be the same - these models are accurate only in case of low steam content (emulsion flow). Higher steam content or stagnant flow causes phase separation and different speeds. The commonly used solution is the so called " $51 / 2$-equation" model - one momentum equation but so called "drift flux model" which allows different speeds for water and steam but handles them with algebraic approximations. The RETINA code (Reactor ThermoHydraulics Interactive) can solve both the 6 and the 51/2 equation systems (Hazi et al, 2001) The most demanding task during the tuning and $V \& V$ of the simulator is to tailor these drift-flux correlations to behave correctly in very different circumstances - stagnant, layered coolant in the pressurizer vessel, isolated loop, etc

We have very different situations - normal operating and transients, stopping, cooling down, heating up, reaching criticality of the reactor, loading up, and separating loops with main gate valves (a rare feature possible only for Soviet/Russian reactors). There real art during the $\mathrm{V} \& \mathrm{~V}$ of the models lies in handling and tuning of the drift flux correlations (nodes above each other, separating, nodes horizontally following each others, pipes and stagnant coolants in vessels, etc.)

As non-condensable gases are carried with steam, boron acid solvent (used to absorb neutrons) and radio-nuclides carried with water (in case of leakage) are simulated, too, but 
this task is much simpler than the correct simulation of the two-phase-flow. (However, "aging", decaying radio-nuclides continuously are changing the concentration of different isotopes and this has to be taken into account, too.)

The simulation of the normal operating modes of the power plant are the less demanding for the stability of the numerical integration of the models, and in the same time we have ample data and recordings to fit the parameters of our models. On the other hand, the training of the operators to anticipated (but rarely happening) transients and accidents is the most important and valuable, but these are difficult physical states with no data (so far, so good) to verify and validate the simulation.

The scope of simulation - by definition of the full-scope, replica simulators - covers anything that can happen to the filled-up primary circuit with the closed reactor vessel. (Up to now we do not simulate open reactor vessel with interrupted circulation, as it is usual during the refueling outage.)

Covering only the events happening to the hermetically closed primary circuit (with pipe breaks and loss of coolant accident of course) that means:

- normal operation with normal transients (load changes, frequency regulation)

- bringing to power (heating up, reaching criticality with the reactor, producing steam, speeding and heating up the turbines, reaching the nominal power

- stopping the reactor, cooling down, changing to natural circulation without the pumps, etc.

- malfunctions of any kind, pump trips, valve jams, valve leaks, short circuits, failures of electrical supplies, island mode of operation (separation from the electrical grid)

- $\quad$ accidents up to the design basis accident, which means a large-break LOCA - breaking of the pipe of the pipeline of the main cooling loop.

According to the integration of the models described above, all these transients can be studied in "4D" in the reactor - that means in time and space, too.

\section{Conclusions}

Simulation is not a profession: it is a way of life - I was told on my first international conference I participated - 'Simulation 1977' in Montreaux, Swiss. I can add to that after 34 years: it is a way of thinking, too. The first Reference shows the first book I had to study after I started with simulation in 1970 (Ralston, 1965).

My greatest mentor, Assoc. Prof. Dr. Richard Zobel retired, and went out to his garden with a pint of beer. The next day he started to model and simulate the sounds produced by the small plashing waterfall in the corner of his garden, with great success, resulting in excellent papers. After being invited later as Assoc. Professor in the Prince of Songkla University, Hat Yai and Phuket Campuses, Thailand, where he survived the big tsunami in the Indian Ocean on 2004, he became one of the leading experts in tsunami simulation. Dr. Zobel is going definitely to model and simulate things up to the last minute of his life.

At the beginning the simulation was very different from the simulation we are making today. We had no powerful digital computers available that time or they were used for different purposes secretly - ordinary scientists had no access to them. We used sometimes analogue computers with integrators made from operational amplifiers. Sometimes they were not electrical, but pneumatic 'operational amplifiers'. Modeling meant not always mathematical modeling - we had to use e.g. electrical analogy, representing long pipelines by inductance, vessels and tanks by electrical capacity; pressure meant voltage and flow was represented by electrical current. 
Numerical analysis was very different when the maximal calculating capacity was represented by desktop calculators, first mechanical and after a while electrical. Methods of Runge-Kutta, Fowler-Warten, Hindmarsch-Gear were studied and used widely, together with the flourishing predictor-corrector multistep methods. Everybody had his/her favorite numerical integrating algorithm and praised it to the others.

Nevertheless, even that time and ever since simulation is a great way of learning: Observing a natural phenomenon we gain an imagination how it works and try to build a model selecting the most dominant processes of it. Using powerful computers in a proper way we can learn whether our imagination was good or wrong, or just not enough: something is still missing. Finally, if the results of simulation are really very close, very similar to the real behavior of the studied phenomenon, we get the unforgettable feeling: we are able to understand and describe what Mother Nature had been doing and how!

Back to the nuclear industry, it is obvious that power generating nuclear power plants cannot be used as test facilities to check out different new ideas. (Some people do not like even the doctors "practicing" - they should not practice, they should already know what they are doing before treating a patient.) As matter of fact, simulation is taking all over - working on models is much safer and much cheaper than doing anything else.

The practice of modeling nuclear power plants show that the up-to-date and state-of-art modeling techniques are fully adequate to support all tasks of design, licensing, construction and operation of nuclear power generating plants or other nuclear facilities. Even the cause and the circumstances of different accidents can be determined the best and easiest way by simulation studies.

Simulation is widely used by students of the universities, by design institutes and companies, by the authorities, by research institutes, during the construction and start-up of new nuclear power plants, designing re-fueling, and keeping up the knowledge of experienced operators and for teaching the new ones. Normally, new plants already have the simulator before the real construction is going to be started. (They should be always slightly modified and adjusted to the local circumstances, anyhow. There are no units being exactly identical to each other.)

We are operating and continuously developing the Paks NPP's full-scope replica simulator already 23 years. We have been able to replace the Reactor Protection System, to develop different enhancements to the technology of the NPP and study spatial behavior of very different mixed cores using this simulator successfully. Originally the simulator was called as the '5th unit', because all changes of the four energy generating units had to be performed later to the model system of the simulator, too. Now the simulator became the '1st unit', because any enhancement, development or change has to be demonstrated on the simulator first before getting the approval to do so on the real units, too.

The simulator is busy working in two shifts to teach and keep up the knowledge of the operating personnel of the NPP. It is very difficult to obtain simulator time for other purposes. The most expensive part of the training simulator is the Control room and the corresponding real-time I/O interface to it. Replacing the Control room with a couple of high resolution touch-screens we will be able to reproduce it in several copies, that way to make it affordable for different studies and planned refurbishments, and for teaching students and non-operative personnel, too. Having multiple copies definitely increases the quality of service and support to the operation of our nuclear power plant, producing close to $40 \%$ of electricity of our country. 


\section{Acknowledgment}

I would like to explain my gratitude to my dear colleagues; the advice and help of them during the last 30 years was indispensable to achieve our goals. Special thanks to Laura Bürger, Endre Vegh (both already retired), to Katalin B. Szabó, Dr. Gábor Házi and József Páles for their permanent support. On the international scene I can thank a lot for the valuable support and encouragement of Prof. Dr. Richard Zobel (now retired) and Prof. Agostino Buzzone.

All of the works described above could not be successfully accomplished without the brilliant knowledge and grateful assistance of the instructors and other personnel of the Paks full-scope replica simulator. The help and cooperation of György Nagy, László Dercze, Sándor Borbély, Sándor Czekmeister, József Göttli and others was essential to achieve these results.

\section{References}

Anthony Ralston: A First Course in Numerical Analysis. Published by McGraw Hill Inc., 1965. Hungarian translation: Múszaki Könyvkiadó, 1969.

Gábor Házi, Gusztáv Mayer, István Farkas, Péter Makovi and A. A. El-Kafas: "Simulation of a small loss of coolant accident by using RETINA V1.0D code", Annals of Nuclear Energy, Volume 28, Issue 16, November 2001, Pages 1583-1594

István Farkas, Gábor Házi, Gusztáv Mayer, András Keresztúri, György Hegyi and István Panka, "First experience with a six-loop nodalisation of a VVER-440 using a new coupled neutronic-thermohydraulics system KIKO3D-RETINA V1.1D" Annals of Nuclear Energy, Volume 29, Issue 18, December 2002, Pages 2235-2242

Janos Sebestyen Janosy: Modeling and Simulation of Nuclear Energy in Eastern Europe. Business and Industry Simulation Symposium, 2003 Advanced Simulation Technologies Conference, Orlando, Florida, March 30 - April 03, 2003, ISBN 1565552636

A. Keresztúri, Gy. Hegyi, Cs. Maráczy, I. Panka, M. Telbisz, I. Trosztel and Cs. Hegedús, Development and validation of the three-dimensional dynamic code - KIKO3D, Annals of Nuclear Energy Volume 30 (2003) pp. 93-120.

Janos Sebestyen Janosy: Simulation Aided Instrumentation and Control System Refurbishment at Paks Nuclear Power Plant. First Asian International Conference on Modeling and Simulation, AMS 2007, 27-30 March 2007, Phuket, Thailand, ISBN 0769528457

Janos Sebestyen Janosy: Simulators and Simulation used in Nuclear Power Plant Related Projects. Keynote speech, CUTSE 2007 Curtin University of Sarawak Engineering Conference, 26-27 November, 2007, Miri, Sarawak, Malaysia.

Janos Sebestyen Janosy: Simulators are the key for large-scale Instrumentation and Control System Refurbishment Projects. Keynote speech, Second Asian International Conference on Modeling and Simulation, AMS 2008, May 12-15, 2008, Kuala Lumpur, Malaysia. 


\title{
Safety Studies and General Simulations of Research Reactors Using Nuclear Codes
}

\author{
Antonella L. Costa ${ }^{1}$, Patrícia A. L. Reis ${ }^{1}$, \\ Clarysson A. M. Silva1, Claubia Pereira ${ }^{1}$, Maria Auxiliadora F. Veloso', \\ Bruno T. Guerra ${ }^{1}$, Humberto V. Soares ${ }^{1}$ and Amir Z. Mesquita ${ }^{2}$ \\ Departamento de Engenharia Nuclear - Escola de Engenharia \\ Universidade Federal de Minas Gerais \\ Instituto Nacional de Ciências e Tecnologia de Reatores Nucleares Inovadores/CNPq \\ ${ }^{2}$ Centro de Desenvolvimento da Tecnologia Nuclear/Comissão Nacional de Energia \\ Nuclear - CDTN/CNEN \\ Brasil
}

\section{Introduction}

Interest in safety issues of nuclear research reactors is nowadays increasing due their enlarged commercial exploitation commonly directed at neutrons generation for several types of scientific and social purposes. Power generation is not the main activity of a nuclear research reactor reaching maximum power operation of about $100 \mathrm{MW}$. In spite of this, specific features are necessary to ensure safe utilization of such installations. Therefore, several codes have been used focusing special attention for research reactors safety analysis and valuation of specific perturbation plant processes. A combination of codes for thermal hydraulic analysis, for assessment of probabilistic risk, fuel investigation and reactor physics studies are fundamental tools for an appropriate reactor behaviour definition.

It is appropriate to use internationally recognized, accepted and validated best estimate codes. The continuous development and validation of the nuclear codes ensures the improvement of best estimate methods. Typically, thermal hydraulic system codes may need the most effort in terms of developing input models for system analyses in research reactors. The fuel codes can be used for analysis of design basis accident conditions and may be used to provide initial conditions for the system thermal hydraulic codes. Neutron kinetic codes can be coupled to thermal hydraulic system codes to provide a more realistic simulation of transients where there is a large reactivity variation. Reactor physics codes are typically used to support the performance of the core as well as to provide results used in the system thermal hydraulic codes for accident analysis. Containment codes may be necessary to estimate parameters as the time of failure of the containment, confinement or reactor building.

In this Chapter, the state-of-the-art related to nuclear codes applied to research reactors are being presented. Results of simulations performed with two specific codes, the thermal hydraulic RELAP5 code and the General Monte Carlo N-Particle Transport code (MCNP), for the TRIGA IPR-R1 research reactor in Brazil are also presented. 


\section{Nuclear research reactors in operation}

The main activity of the nuclear research reactors is not connected to power generation. However, they are widely used to several activities as to non-destructive materials testing, radioisotopes production, nuclear medicine, research, and many others fields.

The Research Reactor Database (RRDB) of the International Atomic Energy Agency (IAEA) contains administrative, technical and utilization information on over 670 research reactors including critical and sub-critical assemblies in 69 countries and the European Union. Second the RRDB data, nowadays there are 239 research reactors in operation around the world (see Table 1). Approximately, half of this total is now over 40 years old being necessary to address deficiencies and new requirements that evolve over time. In this way, reactor organizations undertake an array of work activities to either re-establish performance that has degraded over time, maintain performance in the face of changing conditions or adapt to new customer or regulatory demands (IAEA, 2009).

\begin{tabular}{|l|l|l|}
\hline Status & Developed Countries & Developing Countries \\
\hline Cancelled & 1 & 4 \\
\hline Operational & 148 & 91 \\
\hline Shut down & 183 & 21 \\
\hline Decommissioned & 194 & 16 \\
\hline Temporary shutdown & 8 & 5 \\
\hline Planned & 1 & 1 \\
\hline Under construction & 2 & 1 \\
\hline Unverified information & 0 & 1 \\
\hline Total & $\mathbf{5 3 7}$ & $\mathbf{1 4 0}$ \\
\hline
\end{tabular}

From IAEA (2011) http:/ / nucleus.iaea.org/RRDB

Table 1. Research reactors in the world

The operating mode as well as the design of research reactors can vary largely differently from the power reactors. The most common design of research reactors is the pool type, where the core is a cluster of fuel elements sitting in a large pool of water. The water in the pool has function of cooling, as well as moderation, neutron reflector and it is able to assure an adequate radioactive shielding. The reactor cooling occurs predominantly by natural convection, with the circulation forces governed by the water density differences. The heat generated from the nuclear fissions can be also removed pumping the pool water through a heat exchanger characterizing a forced cooling.

Some examples of different types of research reactors are listed in Table 2. The TRIGA reactor is the most common design having about 60-100 cylindrical fuel elements with metal cladding enclosing a mixture of uranium fuel and zirconium hydride. The main characteristic of this type of fuel is the prompt negative temperature coefficient that provides safety and automatically limiting the power when excess of reactivity is suddenly inserted. Fig. 1 shows a photography of an upper view of the research reactor type openpool IPR-R1 TRIGA. IPR-R1 is installed at Nuclear Energy Development Centre (CDTN) of Brazilian Nuclear Energy Commission (CNEN), in Belo Horizonte, Brazil. It works at 100 $\mathrm{kW}$ but will be briefly licensed to operate at $250 \mathrm{~kW}$. It presents low power, low pressure, for application in research, training and radioisotopes production. The reactor is housed in a 6.625 meters deep pool with 1.92 meters of internal diameter and filled with light water. 
Other research reactor designs are moderated using heavy water or graphite. The fast reactors are in a small number; they require no moderator and can use a mixture of uranium and plutonium as fuel. Homogenous type reactors have a core comprising a solution of uranium salts as a liquid, contained in a tank about $300 \mathrm{~mm}$ diameter. This type was popular in the past due to its simple design; however only a small number is nowadays in operation. High temperature research reactors, as that developed in Japan (the HTTR - High Temperature Test Reactor), have mainly the aim of to investigate the TRISO fuel designed for the Generation IV power reactors, as the HTGRs - High Temperature Gas Reactors Verfondern et al., 2007).

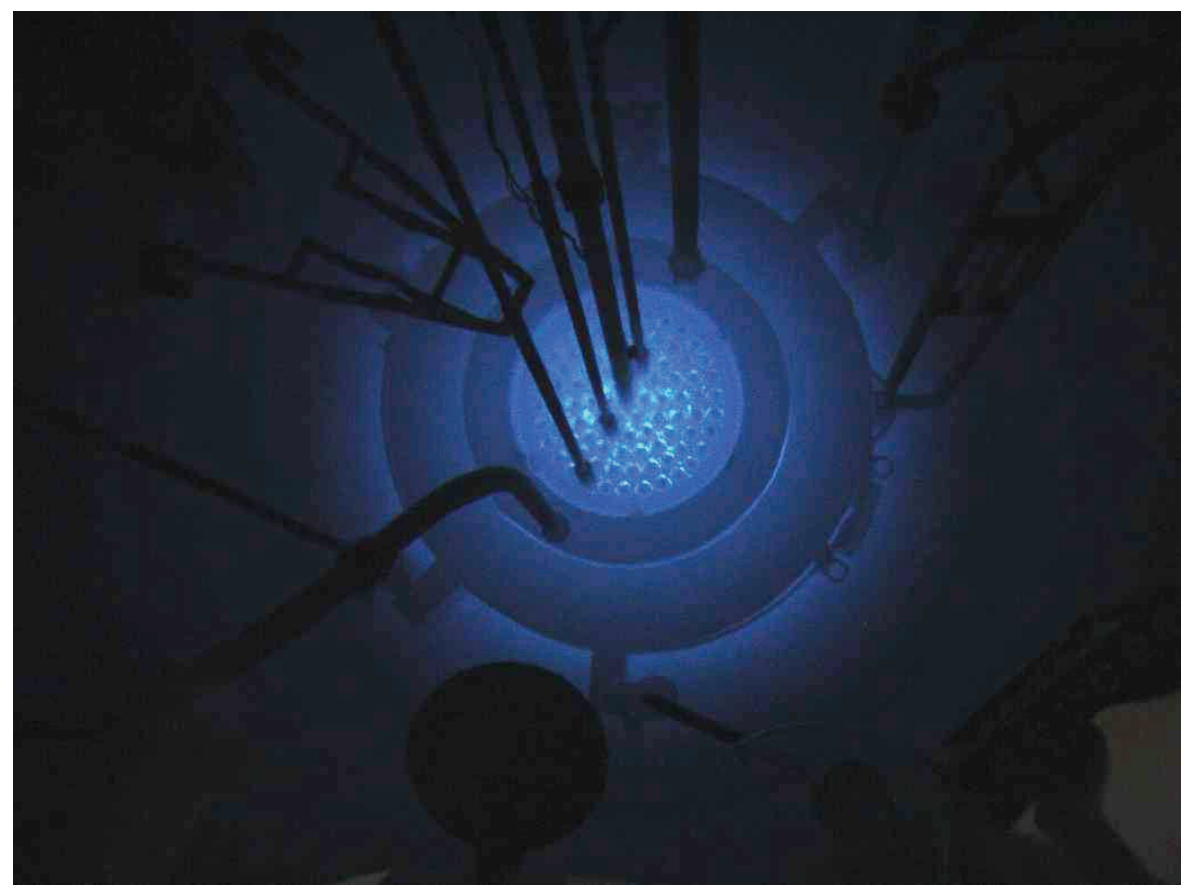

Fig. 1. Core upper view and pool of the IPR-R1 TRIGA

Graphite or beryllium is commonly used as the reflector in research reactors, although other materials may also be used. The fuel of research reactors can be of type HEU (highly enriched uranium) or LEU (low enriched uranium). However, because of the programmes of nuclear non-proliferation, there is a tendency of the countries to convert core reactor HEU to LEU.

The fuel assemblies of research reactors are typically made in plates or cylinders, as presented in the examples of Figure 2 and Figure 3, respectively. Figure 2 illustrates the MTR (material testing reactor) fuel assembly used in the IEA-R1. A typical IEA-R1 fuel element has 18 plane parallel fuel plates, mounted mechanically between two lateral aluminium holders with grooves, and its overall dimensions are $(7.6 \times 8.0) \mathrm{cm}$ and $88.0 \mathrm{~cm}$ high. Each fuel plate consists of an aluminium cladding and a meat where the nuclear fuel is located (Terremoto et al., 2000). 
Figure 3 presents the design of two types of cylindrical fuel elements used in the research reactor IPR-R1. This fuel contains high concentrations of hydrogen using a metal alloy of uranium and zirconium and its main characteristic is the prompt negative temperature coefficient.

\begin{tabular}{|c|c|c|c|c|c|c|}
\hline Type & Name & Country & $\begin{array}{c}\text { Power } \\
(\mathrm{kW})\end{array}$ & Criticality & $\begin{array}{c}\text { Thermal Flux } \\
\left(\mathrm{n} / \mathrm{cm}^{2} \mathrm{~s}\right)\end{array}$ & $\begin{array}{c}\text { Fuel and } \\
\text { Enrichment }\end{array}$ \\
\hline TRIGA & IPR-R1 & Brazil & 100 & 1960 & $4.3 \times 10^{12}$ & $\begin{array}{c}\mathrm{U}-\mathrm{Zr}-\mathrm{H} \\
20 \%\end{array}$ \\
\hline Pool & IEA-R1 & Brazil & 5000 & 1957 & $4.6 \times 10^{13}$ & $\begin{array}{c}\mathrm{U}_{3} \mathrm{O}_{8}-\mathrm{Al} \text { and } \\
\mathrm{U}_{3} \mathrm{Si}_{2}-\mathrm{Al} \\
20 \% \\
\end{array}$ \\
\hline Pool MTR & MNR & Canada & 5000 & 1959 & $1.0 \times 10^{14}$ & $\begin{array}{c}\mathrm{U}_{3} \mathrm{Si}_{2}-\mathrm{Al} \\
19.75 \%\end{array}$ \\
\hline Fast Source & TAPIRO & Italy & 5 & 1971 & $\begin{array}{c}\text { (Fast Flux) } 4.0 \\
\text { x } 10^{12}\end{array}$ & $\begin{array}{c}\text { U-Mo alloy } \\
93.5 \%\end{array}$ \\
\hline $\begin{array}{l}\text { High Tem- } \\
\text { perature Gas }\end{array}$ & HTTR & Japan & 30000 & 1998 & $7.5 \times 10^{13}$ & $\begin{array}{l}\mathrm{UO}_{2} \\
6 \%\end{array}$ \\
\hline Argonaut & UFTR & USA & 100 & 1959 & $2.0 \times 10^{12}$ & $\begin{array}{c}\mathrm{U}_{3} \mathrm{Si}_{2}-\mathrm{Al} \\
19.75 \%\end{array}$ \\
\hline Heavy Water & NBSR & USA & 20000 & 1967 & $4.0 \times 10^{14}$ & $\begin{array}{c}\mathrm{U}_{3} \mathrm{O}_{8}-\mathrm{Al} \\
93 \%\end{array}$ \\
\hline
\end{tabular}

From IAEA (2011) http:/ / nucleus.iaea.org/RRDB

Table 2. Examples of nuclear research reactors

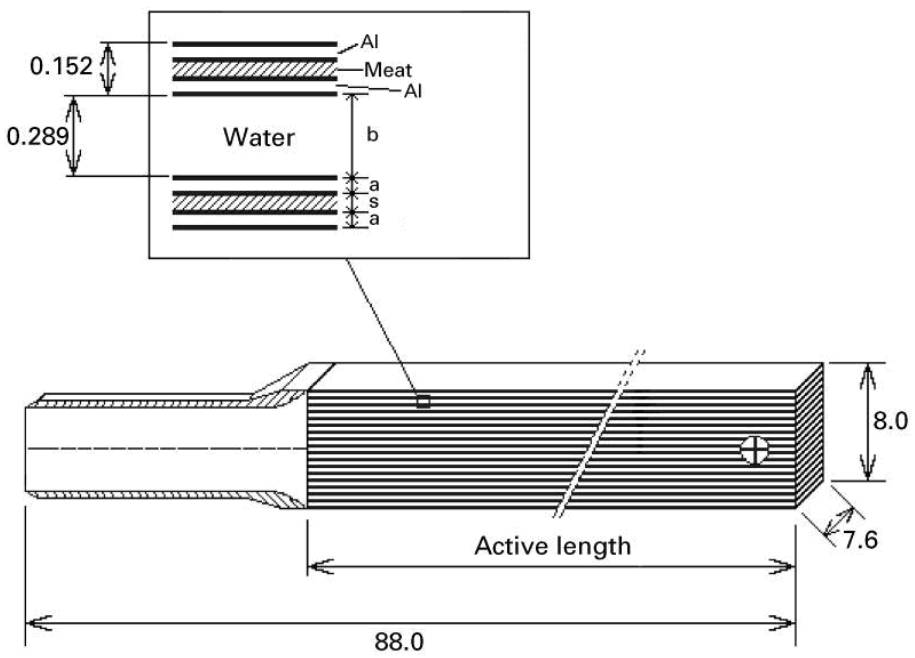

Fig. 2. Cross-sectional diagram of a standard MTR fuel element irradiated in the IEA-R1 research reactor, showing in detail the structure of two successive fuel plates (measure in $\mathrm{cm})$. Adapted from (Terremoto et al., 2000) 

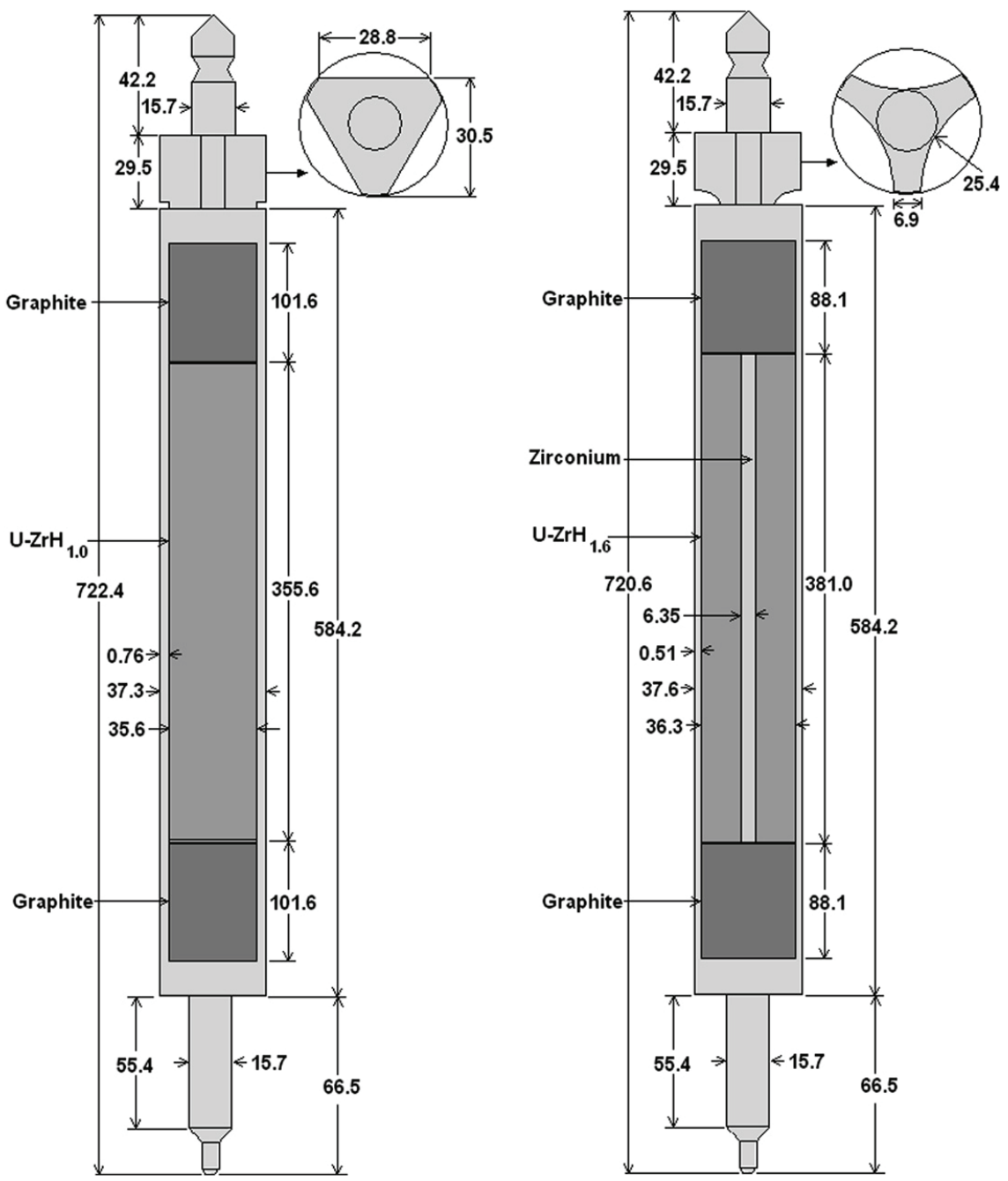

Fig. 3. IPR-R1 TRIGA - design of two types of cylindrical fuel elements (measure in mm)

\section{Application of nuclear codes in research reactor analysis}

In general, the codes used for research reactors analysis are also used in the nuclear power plant (NPP) having both the same basis of development and utilization. The differences on validation and application for each case appear due the complexity of the different classes of reactors. Particularly, the codes available internationally for safety analysis of research reactors can be classified in different issues according with their application including reactor physics, fuel behaviour, thermal hydraulic processing, computational fluid dynamics (CFD) and structural analysis (IAEA, 2008). Each of these topics are being explained with some more details and exemplified next. 


\subsection{Neutron kinetic modeling}

Reactor physics codes are capable to model the 2D or 3D core neutron kinetics for analysing local or asymmetrical effects in the reactor core that is possible to occur as in steady state as in transient operation. Examples of reactor physics codes are WIMS-D, DYN3D, CITATION, PARET and NESTLE. WIMS-D, a deterministic code system for reactor lattice calculation, can be used to calculate group constants dividing the core into several identical unit cells. The calculated cross-sections can then be used as input to another type of code as, for example, the code CITATION for global core calculations (Khana et al., 2000; Dalle et al., 2002).

The computer code DYN3D was developed for safety analyses of nuclear reactors after reactivity perturbations of the system, but it can be used also for fuel management calculations.

PARET code has been used extensively for research reactor analysis which iteratively solves for the neutronic-hydrodynamic-heat transfer aspect of the reactor under steady state and transient behaviour. It can be used to investigate core reactivity insertions being a coupled kinetics and thermal hydraulics code for predicting the course of non-destructive transients in research reactors (Woodruff et al., 1996; Housiadas, 2002; Velit and Primm, 2008).

Other example is the NESTLE code that solves the two or four group neutron diffusion equations in either Cartesian or hexagonal geometry using the Nodal Expansion Method (NEM) and the non-linear iteration technique. NESTLE was embedded in the thermal hydraulic code RELAP5 obtaining the multi-dimensional neutron kinetics model RELAP53D. Steady-state eigenvalue and time dependent neutron flux problems can be solved by the NESTLE code as implemented in RELAP5-3D. In spite of RELAP5-3D to be developed for power reactors applications, it has been successfully used for research reactors analyses (Costa et al., 2011; Marcum et al., 2010).

Therefore, as can be verified from some before examples, generally two or more codes are used directly or indirectly connected for a more detailed and realistic simulation exploring the main capability of each one.

Calculations using discrete ordinate diffusion and transport theory have been used extensively for reactor simulation purposes. However, the Monte Carlo technique offers significant advantages, since the complex geometrical configuration of the reactor core can be modelled in detail. Therefore, the Monte Carlo code (MCNP) has been applied to research reactor simulations mainly for neutron flux calculations (Fernandes et al., 2010; Shoushtari et al., 2009; Stamatelatos et al., 2007; Huda, 2006). A more detailed example of the MCNP code application to simulations of neutron flux value on the irradiation channels of the IPR-R1 TRIGA research reactor, adapted from (Guerra et al., 2011), has been presented in the Annex A.

\subsection{Fuel analysis}

Researchers in several countries have worked with the aiming to develop codes that predict the behaviour of a fuel assembly during extreme transients as, for example, a LOCA (loss of coolant accident). Such codes attempt to predict the deformation of a fuel rod, the termination of deformation by rupture, the temperature reached by the cladding, oxidation of cladding, and in some codes, the interaction between neighbouring rods. Codes which calculate fuel rod behaviour in whole assemblies including rod-to-rod interactions are relatively rare. One example of such code is the Japanese FRETA-B specialised in twodimensional analysis in the transverse direction (NEA, 2009). DRACCAR is other example 
of fuel code that is currently under development at IRSN (Institute for Radiological Protection and Nuclear Safety) with the purpose of to simulate the thermal mechanical behaviour of a rod bundle under LOCA with a 3D multi-rod description (Papin et al., 2006).

\subsection{Thermal hydraulic modeling}

Thermal hydraulic system codes are applicable to a wide variety of reactor designs and conditions. Such system codes allow simulating the complete primary and secondary circuits and the interactions between them. Examples of system thermal hydraulic codes are RELAP5, TRAC, CATHARE, ATHELET, DINAMIKA and CATHENA. They are generally classified as best estimate codes. The term "best estimate code" means that the code is free of deliberate pessimism and contains sufficiently detailed models to describe the relevant processes of the transients that the code is designed to model (IAEA, 2008).

Models for two fluid, non-equilibrium hydrodynamics, point and multidimensional reactor kinetics, control systems, and special system components make these thermal hydraulic codes very attractive. However, the use of these codes for research reactors must be careful in order to ensure that the models included in such codes are valid for the operating regimes of the research reactors. The validity of the models and correlations should be verified.

As an example, the ATHLET thermal hydraulic code developed at the GRS, Society for Plant and Reactor Safety, was planed to analyse leaks and transients for power reactors. However, to extend the applicability of the code to the safety analysis of research reactors, a model was implemented permitting a description of the thermal-dynamic non-equilibrium effects in the subcooled boiling regime (Hainoun et al., 1996).

In the same way, the RELAP5 code has been modified to better simulate the research reactors operation conditions (low pressure, low mass flow rate, low power). For example, a subcooled boiling model of upward vertical flow consistent with phenomenological observations of the subcooled flow boiling mechanisms was proposed to extend the range of applicability of the RELAP5 code to low pressures (Končar and Mavko, 2003). Therefore, recent works as, for example (Antariksawan et al., 2005; Khedr et al., 2005; Marcum et al., 2010; Reis et al., 2010), have been performed to investigate the applicability of the RELAP5 code to research reactors operating conditions (TRIGA 2000, MTR, Oregon State TRIGA, IPR-R1 TRIGA), respectively. Application of a model for the IPR-R1 TRIGA using the RELAP5 code is detailed in the Annex B.

The user of a thermal hydraulic system code has a very large number of available basic elements (single volumes, pipes, branches, junctions, heat structures, pumps, etc) to develop a detailed reactor nodalization. The model can reproduce a specific part or the whole system to be simulated. However as there is not a fixed rule to perform the nodalization, a large responsibility is passed to the user of the code in order to develop an adequate model scheme which makes best use of the various modules and the prediction capabilities of the specific code (Petruzzi and D'Auria, 2008; D'Auria and Galassi, 1998).

Subchannel codes are used to analyse specific processes within the core of the reactor, such as localized flow and heat transfer variables in representative fuel assemblies. Examples are PARET and COBRA codes.

Computational Fluid Dynamics (CFD) is increasingly being used in the nuclear community to model safety relevant phenomena occurring in the reactor coolant system and for the analysis of localized phenomena such as the flow pattern in complex geometries. However, CFD is a relatively recent development and their qualification status for application in transient flow analysis for research reactor licensing should be verified. 


\subsection{Structural codes}

Structural analysis codes are used to describe the behaviour of mechanical components such as core support and pool structures, in the case of a pool type reactor, under various accident conditions. These codes are commercially available and have generally been developed for non-nuclear applications. They utilize boundary conditions supplied, for example, by thermal hydraulic codes. Examples of structural analysis codes are NASTRAN and ANSYS. NASTRAN, the NASA Structural Analysis System, is a powerful general purpose finite element analysis program and it is a standard in the structural analysis field, providing the engineer with a wide range of modelling and analysis capabilities. The computational programme ANSYS is a multipurpose finite element code that can perform a variety of calculations, including stress analysis, temperature distributions, and thermal expansions in solid materials.

\section{Verification and validation of codes}

The applicability of a code to reactor safety analysis, mainly for licensing, is directly related with its qualification which must be rigorously documented. It is not possible to provide a detailed list of the key phenomena and code features necessary for each type of code. However, the IAEA proposes basically three criteria to verify the adequacy of the codes for treating important phenomena (IAEA, 2008):

a. The use of internationally recognized and accepted codes provides some assurance that the codes are adequate for their intended application.

b. Individual codes need to be evaluated on a systematic basis, comparing the intended application of the code with the actual conditions for which the code is applied.

c. Lists of important phenomena expected during the transients that constitute the target of the investigation must be established. In many cases, documentation is available on an individual code basis that describes the relative importance of the different phenomena.

Code verification is defined as the review of the source coding against its description in the documentation. The line by line verification of large codes is a time consuming and expensive process. Therefore, this process is limited to only some codes. However, many industry sponsored codes have been subjected to stringent verification procedures as a consequence of the regulatory licensing process (IAEA, 2008).

Extensive code validation requires efforts at the international level, involving validation projects, usually managed by the code developers and carried out, under cooperation and exchange agreements, by user groups worldwide with access to experimental facilities designed to provide data on behaviour and phenomena of importance. Several international standard problems provide comparison between codes.

The validation of a code modelling for determined system implicates that the model reproduces the measured steady-state conditions of the system with acceptable margins. The nodalization may be considered qualified when it has a geometric fidelity with the system, it reproduces the measured steady-state condition of the system, and it demonstrates satisfactory time evolution conditions ( $\mathrm{D}^{\prime}$ Auria et al., 1999). However, sometimes a nodalization qualified to simulate determined condition may not be suitable to simulate other type of situation being necessary modifications and re-qualification.

Sensitivity analysis including systematic variations in code input variables or modelling parameters, must be used to help identify the important parameters necessary for an accident analysis by ranking the influence of accident phenomena or to bound the overall 
results of the analysis. Results of experiments can also be used to identify important parameters (Reis et al., 2011).

\section{Safety analysis criteria}

The acceptance criteria are essential to classify the results obtained from a safety analysis and they may be specified as basic and specific. Basic acceptance criteria are usually defined as limits set by a regulatory body. The specific acceptance criteria are used to include additional margins beyond the basic acceptance criteria to allow for uncertainties and to provide additional defence in depth. The margin between results predicted by the analysis and the acceptance criterion is related to the uncertainties. If a result has low uncertainty, a small margin to the acceptance criteria may be acceptable. In general, the adequacy of the margin with the acceptance criterion is demonstrated by using a conservative analysis to meet the acceptance criterion (IAEA, 2008).

Deterministic techniques are the main tools used in the analyses of research reactors. These techniques are often related with the conservatism, commonly knew as conservative approach. On the other hand, best estimate method provides a realistic simulation of a physical process to a level commensurate with the currently known data and knowledge of the phenomena concerned. A best estimate analysis must be supplemented by an uncertainty analysis. Application of best-estimate (realistic) computer codes to the safety analysis of nuclear plants implies the evaluation of uncertainties. This is connected with the (imperfect) nature of the codes and of the process of codes application. The source of uncertainties affects the predictions by best-estimate codes and must be taken into account (D'Auria, 2004).

\section{Reactor parameters in the safety analysis}

The safety analyses are used in several areas including design, licensing, support for accident management and emergency planning. Reactor parameters and some operating conditions considered in the safety analysis can be summarized in: state of the reactor operation, core power, core inlet temperature, fuel element cladding temperature; system pressure, core flow, axial and radial power distribution and hot channel factor; reactor kinetics parameters, fuel and moderator temperature reactivity coefficients, void reactivity coefficient, available shutdown reactivity worth and insertion characteristics of reactivity control and safety devices.

The evaluation of the safety of research reactors includes firstly the determination of the reactor response to a range of postulated initiating events (PIEs) covering all supposed possible types of events. Several selected PIEs for research reactors have been classified and summarized as follows (IAEA, 2005):

a. Loss of electric power supplies;

b. Insertion of excess reactivity;

c. Loss of flow;

d. Loss of coolant;

e. Erroneous handling or failure of equipment;

f. Special internal events;

g. External events;

h. Human errors. 
The PIEs in each group should be evaluated to identify the events that would be limiting, and from there the events selected for further analysis should be indicated. Such events would include those having potential consequences that bound all other PIEs in the group. For example, to TRIGA reactors, due the passive nature of the reactivity feedback during a temperature excursion, few PIEs would be applied since any increase in core temperature has a negative reactivity effect, causing a passive reduction in reactor power to limit a temperature excursion reactor. In spite of this, some perturbation situation may occur disturbing the normal reactor operation, as a condition of forced recirculation off. The event can be caused by the recirculation pump failure e can be classified inside the event number 3 described before. Annex B presents an example of simulation of this event using a TRIGA model in the RELAP5 code with results very approximate from the experimental data.

\section{Conclusion}

This Chapter has drawn the attention to specific features related to the safe utilization of research reactors. A summarized state-of-the-art about research reactors was presented.

As it was illustrated, the rising interest in the commercial exploitation of these types of nuclear reactors is justified by the several applications using mainly their neutrons generation. Due the considerable age of the majority of research reactors in operation around the world, it is necessary to address deficiencies and new requirements that evolve over time. Works have been performed with the aim of either re-establish performance that has degraded over time, maintain performance in the face of changing conditions or adapt to new customer or regulatory demands. As part of these efforts, several codes have been used focusing special attention for the research reactors safety analysis including thermal hydraulic analysis, fuel investigation, reactor physics and structural studies. Codes internationally recognized, accepted and validated are essential in reactor safety analysis that are used in several areas including design, licensing, support for accident management and emergency planning.

An important aspect in the applicability of a code to reactor safety analysis is its direct relation with its qualification which must be rigorously documented. Moreover aspects connected to the validation, uncertainty analysis and sensitivity analysis must be carefully considered for a correct application of a nuclear code for the simulation of reactor model.

Examples of application of two types of codes widely used for research reactors analysis are being presented in the Annex A and B (MCNP and RELAP5 codes, respectively). These Annexes present results of simulations performed at the Nuclear Engineering Department of the Federal University of Minas Gerais for the TRIGA IPR-R1 research reactor in Brazil.

\section{Acknowledgment}

The authors would like to thank to Nuclear Technology Development Centre/Brazilian Nuclear Energy Commission (Centro de Desenvolvimento da Tecnologia Nuclear/Comissão Nacional de Energia Nuclear - CDTN/CNEN), Research Support Foundation of the State of Minas Gerais (Fundação de Amparto à Pesquisa de Minas Gerais - FAPEMIG), Brazilian Council for Scientific and Technological Development (Conselho Nacional de Desenvolvimento Científico e Tecnológico - CNPq) and the Coordination for the Improvement of Higher Education Personnel (Coordenação de Aperfeiçoamento de Pessoal de Nível Superior - CAPES) for the support. 


\section{ANNEX A. Example of MCNP code application to IPR-R1 research reactor}

The Annex A and also the Annex B are based in the IPR-R1 research reactor and they are a summary of the works (Guerra et al., 2011) and (Reis et al., 2010), respectively.

\section{Al. Introduction}

The IPR-R1 is a reactor type TRIGA, Mark-I model that is installed at Nuclear Technology Development Centre (CDTN) of Brazilian Nuclear Energy Commission (CNEN), in Belo Horizonte, Brazil. It is a light water moderated and cooled, graphite-reflected, open-pool type research reactor. IPR-R1 works at $100 \mathrm{~kW}$ but it will be briefly licensed to operate at 250 $\mathrm{kW}$. It presents low power, low pressure, for application in research, training and radioisotopes production. The fuel is an alloy of zirconium hydride and uranium enriched at $20 \%$ in $235 \mathrm{U}$.

The IPR-R1 reactor has a Rotary Specimen Rack, RSR, outside the reactor, and it is composed by forty irradiation channels in a cylindrical geometry. Moreover, tangent to annular reflector, there is a Pneumatic Tube where the samples also can be inserted to irradiation. Therefore, the IPR-R1 has three facilities for sample irradiation: the Central Thimble, the Rotary Specimen Rack and the Pneumatic Tube. Figure A1 shows the radial and axial core configuration. The IPR-R1 has the main nuclear applications related with neutron activation analysis (NAA), training of operators for nuclear power plants and experiments in neutronic and thermal hydraulic.
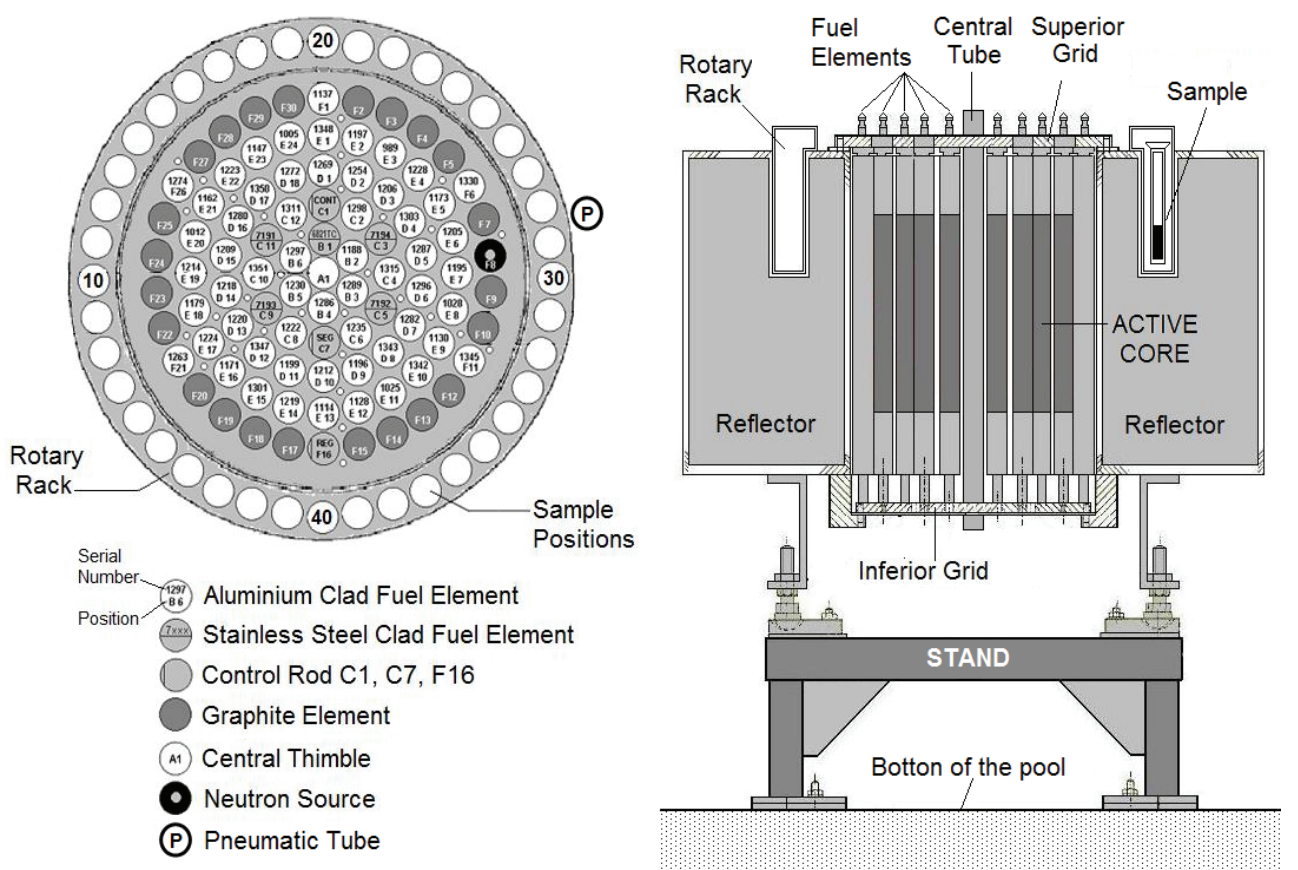

Fig. A1. Core upper view and pool of the IPR-R1 TRIGA 
The IPR-R1 has been simulated using the code MCNPX2.6.0 (Monte Carlo N-Particle Transport eXtend). The goal was to evaluate the neutronic flux in a sample inserted in the RSR channels. In each simulation the sample was placed in a different position, totalling forty positions around the RSR. The results obtained from the calculation show good agreement in relation to experimental data.

\section{All. Modelling}

The reactor model was developed using MCNP5 and MCNPX codes where 500 active cycles were calculated with 1000 neutrons per cycle. The simulations executed in MCNPX code, considers 1.0 hour of irradiation (time of sample irradiation) with $0.1 \mathrm{MW}$ (currently TRIGA reactor power). The simulated model was based on previous studies where the reactor core has the same features of the IPR-R1 geometry described before. The configured geometry is the same to MCNP5 and the MCNPX 2.6.0. The core was configured considering a cylinder containing water, fuel elements, radial reflectors, central tube (or central thimble), control rods and neutron source. Each rod has a coordinate value. They were filled according to their individual characteristics. Around the core there is the RSR which has groove to insert the samples to irradiation. The configured core is inside the pool where water surrounds the core and the RSR. However, the model of this work presents some improvements.

Table A1 presents the cylinders dimensions which are inside of RSR. In addition, Figure A2 illustrates the axial and radial view of the simulated model.

\begin{tabular}{|l|l|l|l|}
\hline Cylinder Material & Inner Radius $(\mathrm{cm})$ & Radial Thickness $(\mathrm{cm})$ & Height $(\mathrm{cm})$ \\
\hline Aluminum & 1.50 & 0.10 & 20.0 \\
\hline Polystyrene & 1.10 & 0.30 & 7.90 \\
\hline Polyethylene & 0.48 & 0.07 & 0.55 \\
\hline
\end{tabular}

Table A1. Dimensions of the simulated cylinders inside Rotary Specimen Rack
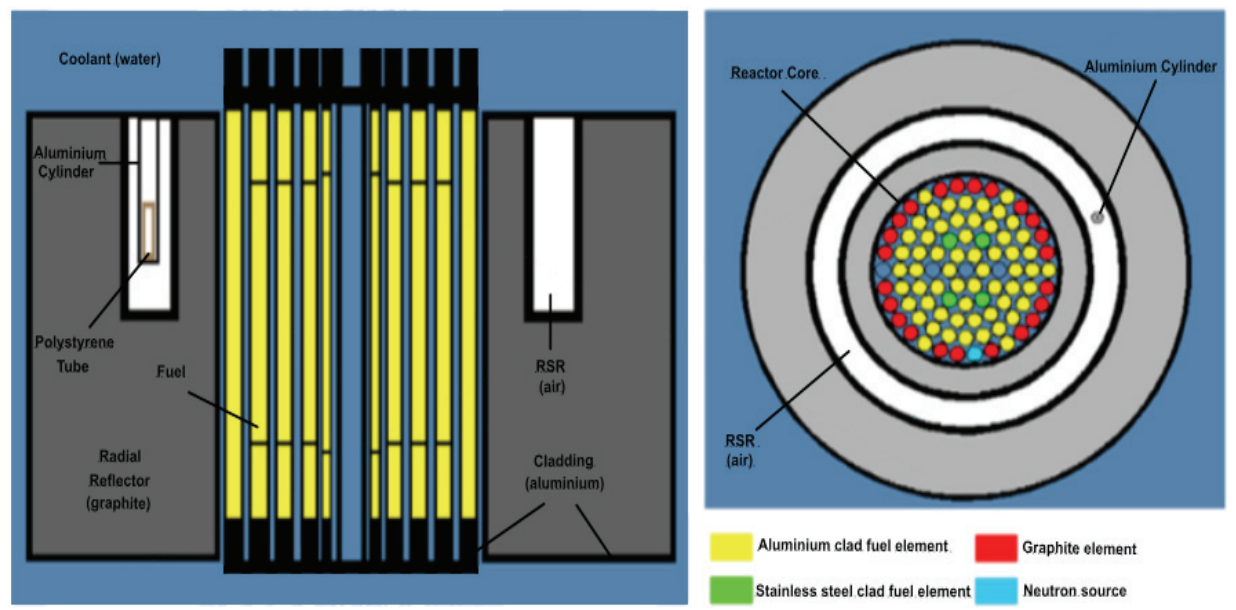

Fig. A2. Axial and radial view of the reactor TRIGA IPR-R1 simulated in MCNP5 and MCNPX codes 


\section{Alll. Results and conclusions}

The assembly of the three cylinders was positioned in 40 different positions of the RSR to calculate the thermal neutron flux (energies of $0.5 \mathrm{eV}$ or smaller) inside of the polyethylene cylinder that contains the sample. The Figure A3 shows the thermal neutron flux simulated by MCNP5 and MCNPX codes. It is possible to see clearly the behaviour of the thermal neutron flux around the core reactor. The neutron fluxes vary at each position and changes over the RSR. In spite of the good agreement of the most of the calculated points some differences were observed. These differences must be justified by a theoretical analysis and using a statistical program.

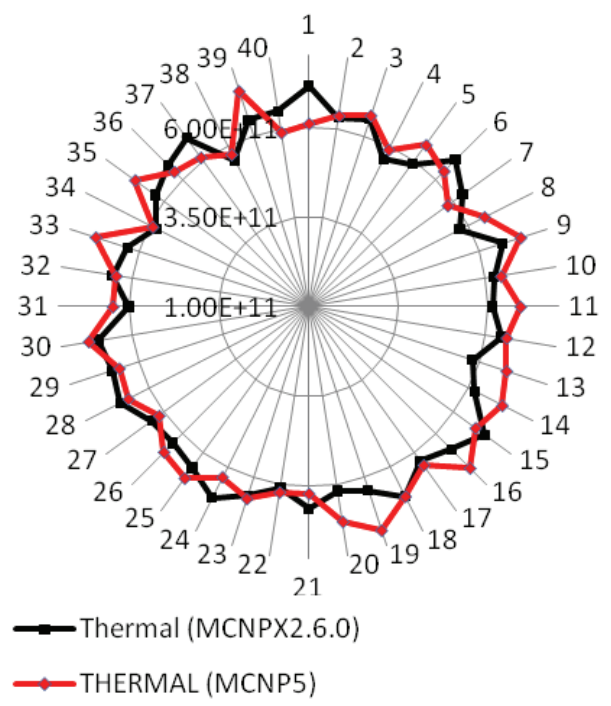

Fig. A3. Axial and radial view of the reactor TRIGA IPR-R1 simulated in MCNP5 and MCNPX codes

Although the neutron flux value was calculated in 40 positions in the RSR, the reference work presents only 11 experimental data. Table A2 presents the values of thermal neutron flux calculated by the codes MCNP-4B, MCNP5 and MCNPX 2.6.0. The results are being compared with experimental data and the error is presented in percentage.

Eight positions present differences smaller than $10 \%$ and three positions present differences smaller than $21 \%$. In this way, it is possible to conclude that the most of the calculated results are in good agreement with the experimental measurements.

The results showed that there is good agreement between the experimental data and the values calculated by the used codes. The errors presented by the most of estimated flux are smaller than $10 \%$ and three positions presented differences between 10 and $21 \%$. The tool employed by MCNP5 and MCNPX 2.6.0 estimate the flux average inside of the cylinder which contains the sample. However, the used codes have other method to calculate the neutron flux as Forced Collision, Point Detectors, Spherical Detector, etc. These tools may improve the results decreasing the differences between experimental data and the calculated values. For more details of this work see (Guerra et al., 2011). 
Future woks will simulate the IPR-R1 employing other method to flux calculate. The information about neutron flux predicted by MNCP5 and MCNPX 2.6.0 can improve NAA where the sample activity can be estimated knowing neutron flux. Furthermore, these codes can characterize the neutron flux in other parts of the reactor where experimental measuring is difficult to be obtained.

\begin{tabular}{|l|l|l|l|l|l|l|l|}
\hline \multirow{3}{*}{$\begin{array}{l}\text { Position } \\
\text { RSR }\end{array}$} & \multicolumn{3}{|l|}{ Previous Studies } & \multicolumn{3}{l}{ Present Study } \\
\cline { 2 - 8 } & \multirow{2}{*}{$\begin{array}{l}\text { Experi- } \\
\text { mental } \\
\text { Value }\end{array}$} & \multicolumn{2}{l}{$\begin{array}{l}\text { Model 1 } \\
\text { (MCNP 4B) }\end{array}$} & \multicolumn{2}{l|}{$\begin{array}{l}\text { Model 2 } \\
\text { (MCNP5) }\end{array}$} & \multicolumn{2}{l|}{$\begin{array}{l}\text { Model 3 } \\
\text { (MCNPX) }\end{array}$} \\
\cline { 3 - 9 } & Value & Error & Value & Error & Value & Error \\
\hline 1 & 6.69 & 6.77 & 1.18 & 6.11 & 8.67 & 7.14 & 6.30 \\
\hline 3 & 6.55 & 6.65 & 1.50 & 6.60 & 0.76 & 6.50 & 0.76 \\
\hline 7 & 6.35 & 6.67 & 4.80 & 5.79 & 8.82 & 6.32 & 0.47 \\
\hline 10 & 5.99 & 6.90 & 13.19 & 6.44 & 6.99 & 6.24 & 4.01 \\
\hline 24 & 6.94 & 6.98 & 0.57 & 6.33 & 8.79 & 6.97 & 0.43 \\
\hline 25 & 6.45 & 6.86 & 5.98 & 6.91 & 6.66 & 6.54 & 1.38 \\
\hline 29 & 7.32 & 6.86 & 6.28 & 6.57 & 10.25 & 6.77 & 7.51 \\
\hline 34 & 7.30 & 6.73 & 7.81 & 5.90 & 19.18 & 5.77 & 20.96 \\
\hline 35 & 7.18 & 6.72 & 6.41 & 7.00 & 2.51 & 6.29 & 12.40 \\
\hline 38 & 6.58 & 6.80 & 3.24 & 5.76 & 12.46 & 5.58 & 15.20 \\
\hline 40 & 6.16 & 6.73 & 8.47 & 5.91 & 4.06 & 6.51 & 5.38 \\
\hline
\end{tabular}

Table A2. Thermal neutron flux $\left(\times 10^{11} \mathrm{n} / \mathrm{cm}^{-2} \mathrm{~s}^{-1}\right)$

\section{ANNEX B. Example of RELAP5 code application to IPR-R1 research reactor}

\section{BI. Introduction}

The RELAP5 system code was developed to simulate transient scenarios in power reactors such as PWR and BWR but recent works have been performed to investigate the applicability of the code to research reactors operating conditions with good results.

Specifically, the TRIGA reactors are constructed in a variety of configurations and capabilities, with steady-state power levels ranging from 20 kilowatts to 16 megawatts offering true "inherent safety". TRIGA is a pool-type reactor that can be installed without a containment building being designed for use by scientific institutions and universities for purposes such as graduate education, private commercial research, non-destructive testing and isotope production.

In the present work, the IPR-R1 TRIGA reactor, Mark-I model, installed in Brazil, in operation since 1960, has been modeled for RELAP5 code with the aim of to reproduce the measured steady-state as well as transient conditions. The development and the calculation for the validation of a RELAP5 model for the IPR-R1 TRIGA research reactor have been presented. The version MOD3.3 was used to perform the simulations. The current results obtained with the developed nodalization demonstrate that the IPR-R1 TRIGA model is representative of the reactor behaviour considering steady-state and transient operation conditions as it is being described in the next sections. 
IPR-R1 presents low power, low pressure, for application in research, training and radioisotopes production. The reactor is housed in a 6.625 meters deep pool with 1.92 meters of internal diameter and filled with light water. A schematic reactor diagram is illustrated in the Figure B1.

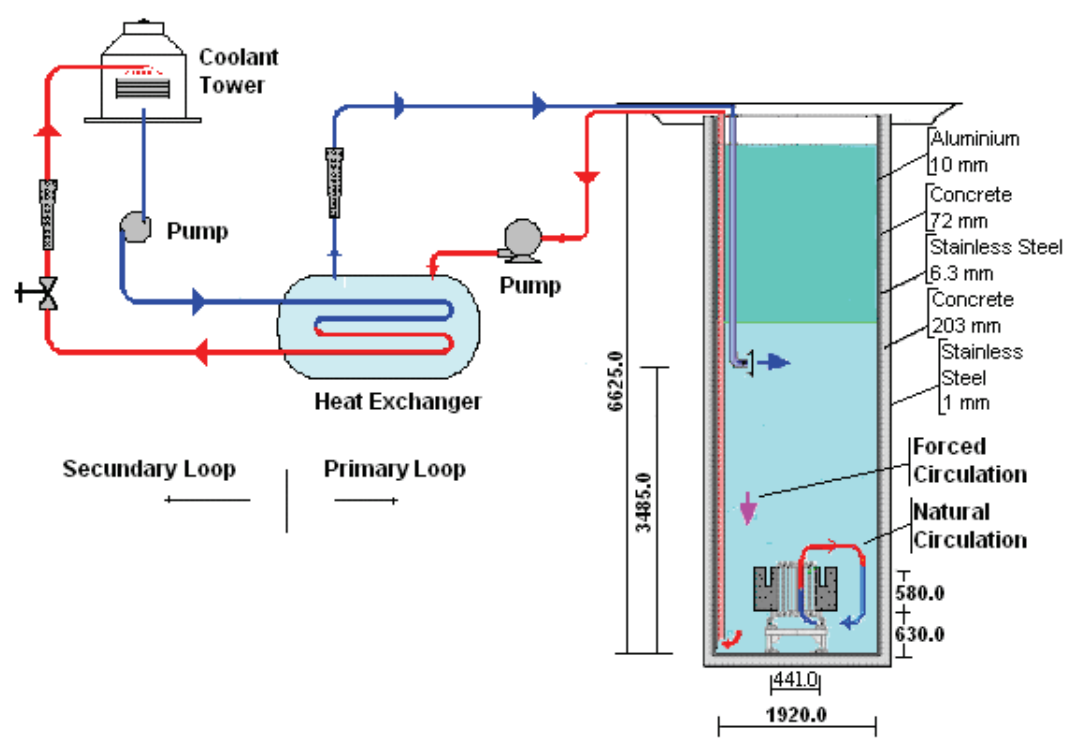

Fig. B1. Schematic representation of the IPR-R1 (out of scale, measure in $\mathrm{mm}$ )

The main aim of the water in the pool is for cooling, as well as moderator, neutron reflector and it is able to assure an adequate radioactive shielding. The reactor cooling occurs predominantly by natural convection, with the circulation forces governed by the water density differences. The heat removal generated from the nuclear fissions is performed pumping the pool water through a heat exchanger. The core has a radial cylindrical configuration with six concentric rings (A, B, C, D, E, F) with 91 channels able to host either fuel rods or other components like control rods, reflectors and irradiator channels. There are in the core 63 fuel elements constituted by a cylindrical metal cladding filled with a homogeneous mixture of zirconium hydride and Uranium $20 \%$ enriched in $235 \mathrm{U}$ isotope. There are 59 fuel elements covered with aluminium and 4 fuel elements with stainless steel.

\section{BII. Modelling}

Each of the 63 fuel elements was modelled separately and 63 heat structure (HS) components were associated with 13 corresponding hydrodynamic pipe components constituting 13 hydrodynamic channels (201 - 213), as can be verified in Figure B2.

Figure B3 shows the RELAP5 general nodalization developed to simulate the IPR-R1. The reactor pool was modelled using two pipe components, each one composed by ten volumes. As it can be verified by the Figure B3, both components (020 and 050$)$ have their volumes connected by single junctions to characterize a cross flow model. This model improves transient predictions as it will be clearly demonstrated in the transient results. A time 
dependent volume was used to simulate the atmospheric pressure on the pool surface. The natural convection system and the primary loop circulation have been modelled. The secondary loop, composed mainly by the external cooling tower was not modelled in the present nodalization because the primary circuit was sufficient to guaranty the heat removal of the coolant.

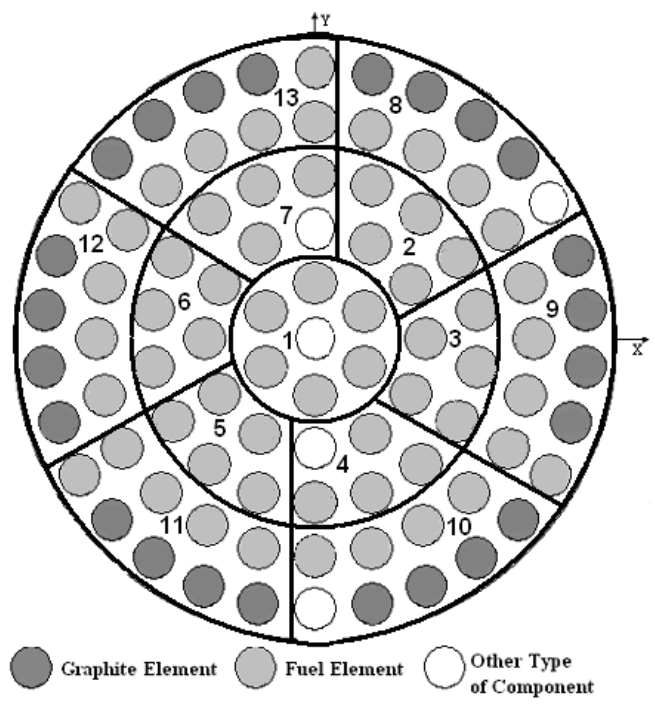

Fig. B2. Representation of the $13 \mathrm{TH}$ channels in RELAP5 model

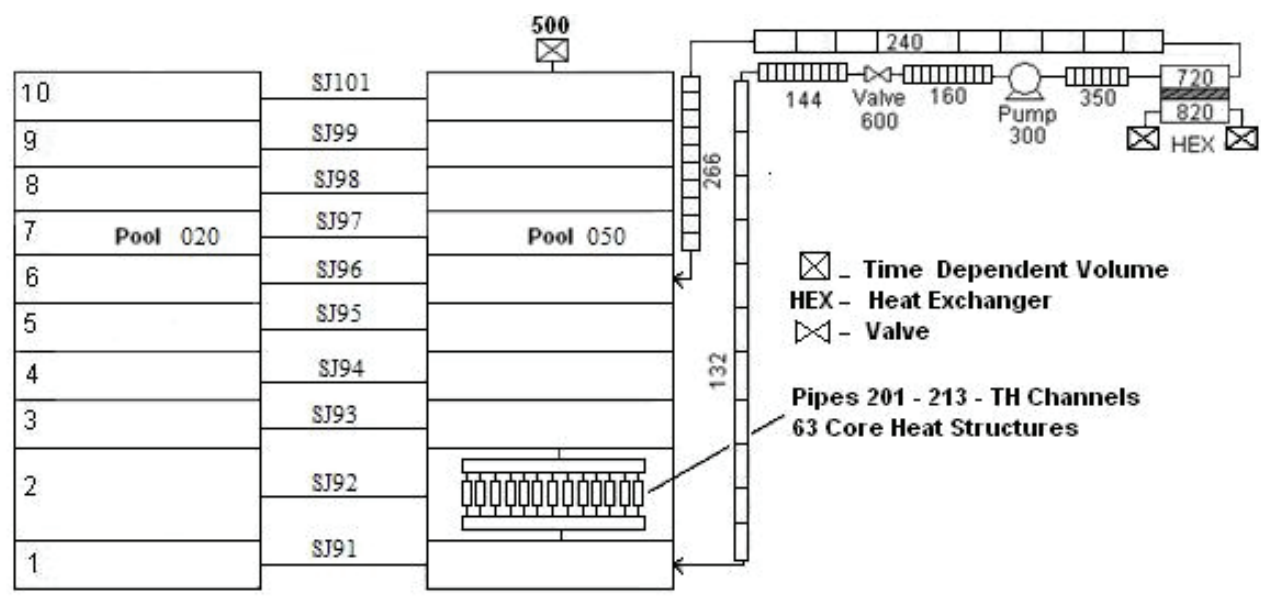

Fig. B3. IPR-R1 TRIGA nodalization in the RELAP5 model

The point kinetics model was used in the current model. A detailed representation of each element is, however, essential to properly take into account the radial power distribution associated with the position of the fuel elements. The axial power distribution was 
calculated considering a cosine profile and taking into account also that the power is cut off in the extremes of the element due the presence of the graphite as it is sketched in the Figure B4. Although the above modelling procedure is approximated, it is used here to maintain the actual axial and radial power distribution fixed.

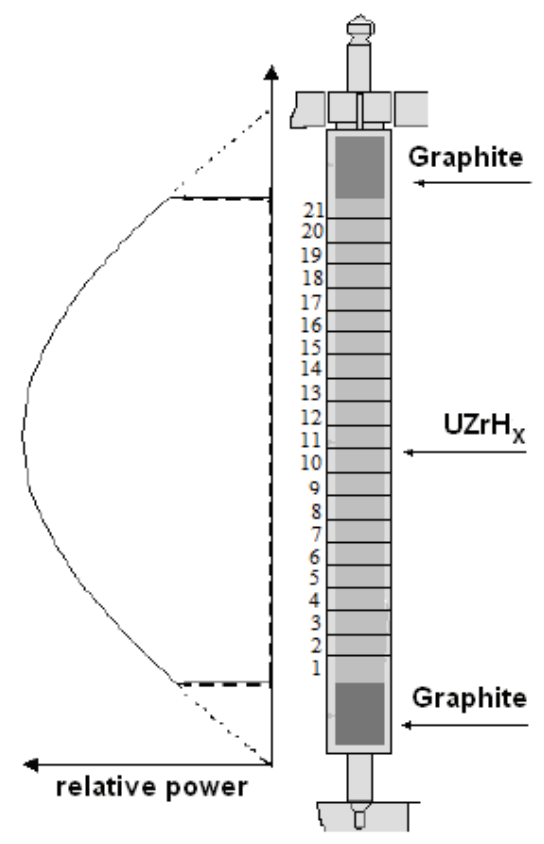

Fig. B4. Prediction of the axial power distribution function in a TRIGA fuel element

\section{BIII. Steady state results}

The validation of a RELAP5 nodalization implicates that the model reproduces the measured steady-state conditions of the system with acceptable margins. The nodalization may be considered qualified when it has a geometric fidelity with the system, it reproduces the measured steady-state condition of the system, and it demonstrates satisfactory time evolution conditions. The RELAP5 steady state calculation has been performed at 50 and 100 $\mathrm{kW}$. The temperature values at the inlet and outlet of the thermal hydraulic channels 3,8 and 13 calculated using RELAP5 can be verified in the Tables B1 and B2, for 50 e $100 \mathrm{~kW}$, respectively. The calculated values were compared with the available experimental data (inlet and outlet channel temperature). Chromel-alumel calibrated thermocouples were used to collect the coolant temperature data and the measured values have a maximum error of $\pm 1^{\circ} \mathrm{C}$.

As it can be verified in the Table B1, considering operation at $50 \mathrm{~kW}$, the results of the RELAP5 code are in good agreement with the experimental data. The error obtained using the RELAP5 calculation is into the range of the maximum acceptable error suggested for coolant temperature $(0.5 \%)$ by the RELAP5 users. 


\begin{tabular}{|c|c|c|c|c|c|c|}
\hline \multirow{2}{*}{$\begin{array}{c}\text { TH } \\
\text { Channel }\end{array}$} & \multicolumn{2}{|c|}{ Outlet Channel Temperature (K) } & \multicolumn{3}{|c|}{ Inlet Temperature (K) } \\
\cline { 2 - 7 } & $\begin{array}{c}\text { Experi- } \\
\text { mental }\end{array}$ & RELAP5 & Error (\%) & $\begin{array}{c}\text { Experi- } \\
\text { mental }\end{array}$ & RELAP5 & Error (\%)* \\
\hline 3 & 300.0 & 298.4 & 0.5 & 294.1 & 294.7 & 0.1 \\
\hline 8 & 298.0 & 296.4 & 0.5 & 296.1 & 294.7 & 0.5 \\
\hline 13 & 298.0 & 296.4 & 0.5 & 0.4 & 294.7 & 0.5 \\
\hline
\end{tabular}

* error $=100 \times($ Calculation - Experimental $) /$ Experimental

Table B1. Experimental and calculated results at $50 \mathrm{~kW}$ of power operation

Results performed at $100 \mathrm{~kW}$ of power operation are shown in Table B2. The error found for RELAP5 calculation is a few overestimated in comparison with the error suggested for coolant temperature $(0.5 \%)$ by the RELAP5 users. However, considering the error from the experimental data $\left( \pm 1^{\circ} \mathrm{C}\right)$ the values predicted using RELAP5 are perfectly acceptable for the present model validation process for operation power up to $100 \mathrm{~kW}$.

\begin{tabular}{|c|c|c|c|c|c|c|}
\hline \multirow{2}{*}{$\begin{array}{c}\text { TH } \\
\text { Channel }\end{array}$} & \multicolumn{2}{|c|}{ Outlet Channel Temperature (K) } & \multicolumn{3}{|c|}{ Inlet Temperature (K) } \\
\cline { 2 - 7 } & $\begin{array}{c}\text { Experi- } \\
\text { mental }\end{array}$ & RELAP5 & Error (\%)* & $\begin{array}{c}\text { Experi- } \\
\text { mental }\end{array}$ & RELAP5 & Error (\%)* \\
\hline 3 & 304.0 & 301.3 & 0.9 & 294.0 & 295.7 & 0.6 \\
\hline 8 & 300.5 & 298.8 & 0.8 & 295.5 & 295.7 & 0.1 \\
\hline 13 & 301.5 & 298.8 & 1.1 & 296.5 & 295.7 & 0.3 \\
\hline
\end{tabular}

* error $=100 \times($ Calculation - Experimental $) /$ Experimental

Table B2. Experimental and calculated results at $100 \mathrm{~kW}$ of power operation

Figures B5 and B6 show the RELAP5 calculation for the inlet and outlet temperature for the TH channel 1, at 50 and $100 \mathrm{~kW}$ of power, respectively. Such channel was chosen because it concentrates the HS with higher values of radial power. As it can be verified, after about $2500 \mathrm{~s}$ of calculation, the temperatures reach steady-state condition. The temperature stable values are in good agreement with the experimental available data.

\section{BIV. Transient results}

In spite of the IPR-R1 to be inherently safe, situations that can disturb the normal reactor operation are possible to occur. The RELAP5 model presented in this work has demonstrated to reproduce very well the steady-state conditions. Therefore, in addition to the validation of the modelling process, a transient event was investigated using the code and the results has been compared with available experimental data. The investigated event is the forced recirculation off and may be caused by the recirculation pump failure, bringing the reactor to operate in natural circulation conditions.

In the experiment, the reactor operated during about 2.5 hours with the forced cooling system switched off and with an indication of $100 \mathrm{~kW}$ at the linear neutronic channel (Mesquita et al., 2009). The measurements have demonstrated an average temperature-rise rate of about $4.8^{\circ} \mathrm{C} / \mathrm{h}$. At inlet and outlet of a thermal hydraulic channel the temperature values were verified to increase about $5.3^{\circ} \mathrm{C} / \mathrm{h}$ in both cases. 


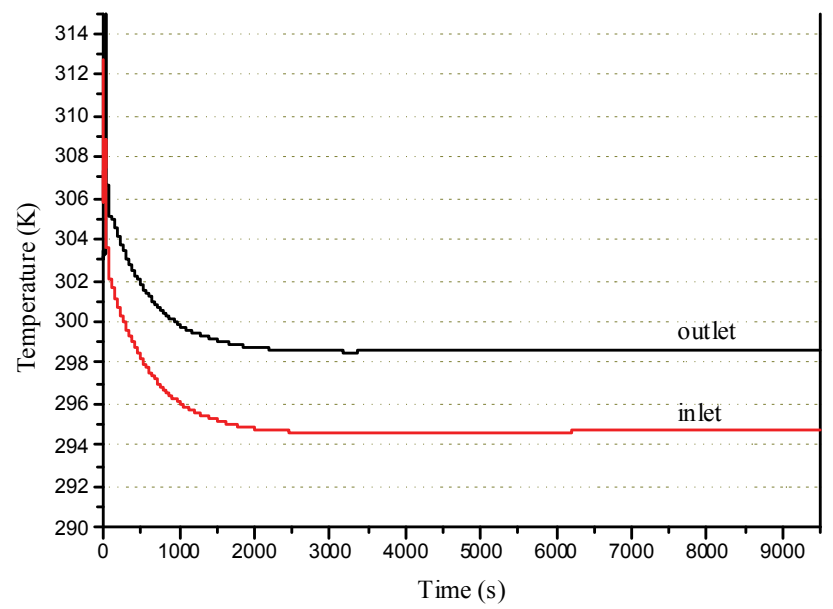

Fig. B5. Inlet and outlet coolant temperature for the channel 1 at $50 \mathrm{~kW}$ predicted by the RELAP5

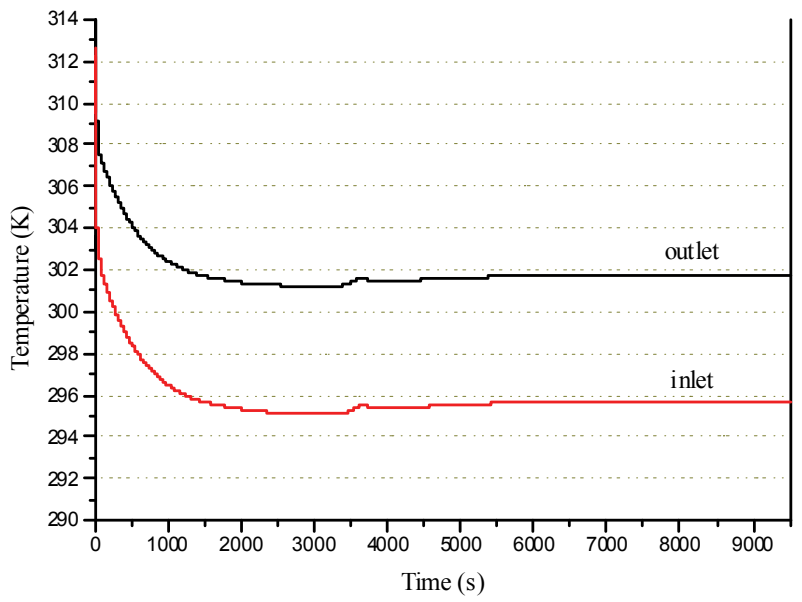

Fig. B6. Inlet and outlet coolant temperature for the channel 1 at $100 \mathrm{~kW}$ predicted by the RELAP5

To perform the simulation using the RELAP5, the valve in the primary system (number 600 in the nodalization) has been closed at $3000 \mathrm{~s}$ of calculation after the system to reach steadystate condition. After the beginning of the transient, the temperatures increase as consequence of no energy removal from the pool since the primary was off (see Figure B7). After the beginning of the transient, the coolant temperature at inlet and outlet TH channel 1 increased gradually with rates of about $4.9^{\circ} \mathrm{C} / \mathrm{h}$ and $4.6^{\circ} \mathrm{C} / \mathrm{h}$, respectively, demonstrating very good agreement with the experimental available data.

The insertion of the cross flow model in the pool nodalization makes possible better removal of heat from the core during natural circulation condition due improvement on the coolant 


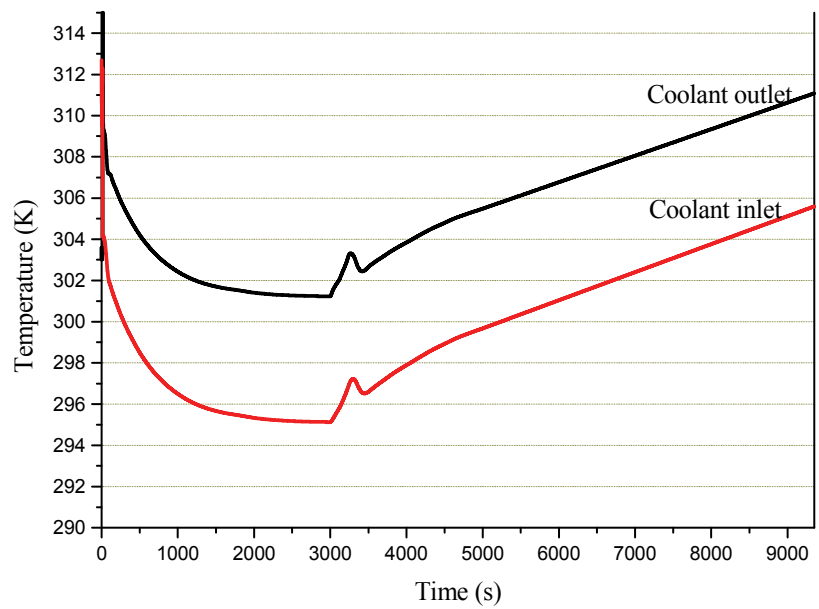

Fig. B7. Inlet and outlet coolant temperature for the channel 1 at $100 \mathrm{~kW}$ predicted by the RELAP5 after forced recirculation off at $3000 \mathrm{~s}$

flow between the pool pipe volumes. Figure B8 illustrates the coolant temperature code prediction considering the nodalization presented in this paper and that in the nodalization without cross flow model, both at $100 \mathrm{~kW}$ of power operation. The curves show clearly that the model using cross flow presents a temperature-rise rate $\left(4.9^{\circ} \mathrm{C} / \mathrm{h}\right)$ much more approximated to the experimental $\left(4.8^{\circ} \mathrm{C} / \mathrm{h}\right)$ than that without cross flow model $\left(30.0^{\circ} \mathrm{C} / \mathrm{h}\right)$.

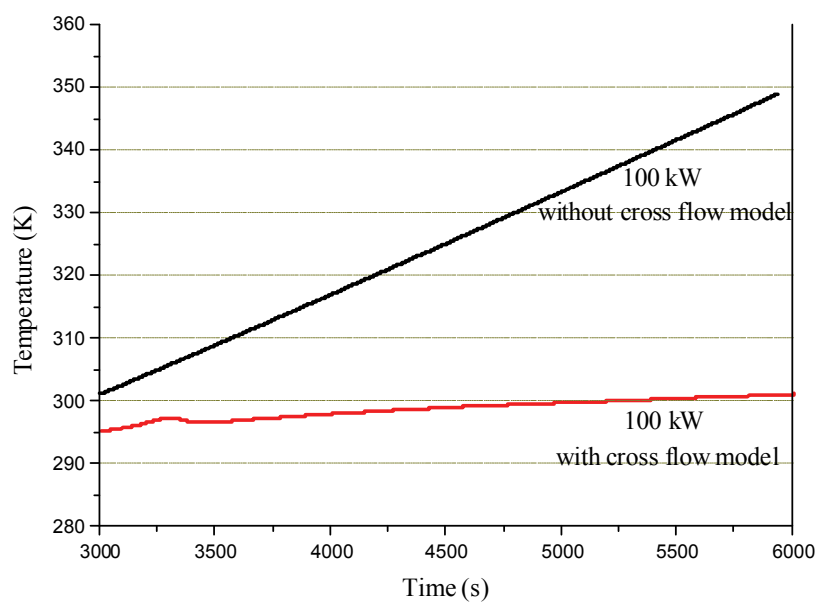

Fig. B8. Forced recirculation off transient prediction using two types of pool nodalization

\section{BV. Conclusion}

Considering the three basic aspects necessary to qualify a nodalization for a system (geometric fidelity, reproduction of the measured steady-state conditions and satisfactory time evolution conditions), it is possible to conclude that the RELAP5 model presented in 
this work was qualified to represent adequately the IPR-R1 TRIGA research reactor in steady-state as well as in transient situations.

\section{References}

Antariksawan, A. R., Huda, M. Q., Liu, T., Zmitkova, J., Allison C. M., Hohorst, J. K. (2005). Validation of RELAP/SCAPSIM/MOD3.4 for research reactor applications, In: 13th International Conference on Nuclear Engineering, pp. 1-8, Beijing, China, May 16-20, 2005.

Costa, A. L., Reis, P. A. L., Pereira, C., Silva, C. A. M., Veloso, M. A. F., Mesquita, A. Z. (2011). Simulation of the TRIGA IPR-R1 research reactor using the RELAP5-3D, Proceedings of the European Research Reactor Conference 2011, pp. 1-5, Rome, Italy, March 20-24, 2011.

Costa, A. L., Reis, P. A. L., Pereira, C., Veloso, M .A. F., Mesquita, A. Z., Soares, H. V. (2010). Thermal hydraulic analysis of the IPR-R1 research reactor using a RELAP5 model. Nuclear Engineering and Design, Vol. 240, pp. 1487-1494.

Dalle, H. M., Pereira, C., Souza, R. G. P. (2002). Neutronic calculation to the TRIGA Ipr-R1 reactor using the WIMSD4 and CITATION codes. Vol. 29, Annals of Nuclear Energy, pp. 901-912.

D'Auria, F. and Galassi, G. M. (1998). Code validation and uncertainties in system thermalhydraulics. Progress in Nuclear Energy, Vol. 33, pp.175-216.

D'Auria, F., Frogheri, M. and Giannoti, W. (1999). RELAP5/MOD3.2 Post test analysis and accuracy quantification of lobi test BL-44. International Agreement Report, NUREG/IA-0153.

D'Auria, F. (2004). Approach and methods to evaluate the uncertainty in system thermalhydraulic calculations. In: Mecánica Computacional, G. Buscaglia, E. Dari, O. Zamonsky (Eds.), Vol. XXIII, pp. 1411-1425, Bariloche, Argentina.

Fernandes, A. C., Santos, J. P., Marques, J. G., Kling, A., Ramos, A. R., Barradas, N. P. (2010). Validation of the Monte Carlo model supporting core conversion of the Portuguese Research Reactor (RPI) for neutron fluence rate determinations. Annals of Nuclear Energy, Vol. 37, pp. 1139-1145.

Guerra, B. T., Silva, C. A. M., Oliveira, A. H., Pereira, C., Costa, A. L. (2011). Simulation of the thermal neutron fluxes characterization in the irradiation channels of the IPRR1 TRIGA research reactor using Monte Carlo method, Proceedings of the European Research Reactor Conference 2011, pp. 1-5, Rome, Italy, March 20-24, 2011.

Hainoun, A., Hicken, E., Wolters, J. (1996). Modelling of void formation in the subcooled boiling regime in the ATHLET code to simulate flow instability or research reactors. Nuclear Engineering and Design, Vol. 167, pp. 175-191.

Housiadas, C. (2002). Lumped parameters analysis of coupled kinetics and thermalhydraulics for small reactors. Annals of Nuclear Energy, Vol. 29, pp. 1315-1325.

Huda, M. Q. (2006). Computational analysis of Bangladesh 3 MW TRIGA research reactor using MCNP4C, JENDL-3.3 and ENDF/B-Vl data libraries. Annals of Nuclear Energy, Vol. 33, pp. 1072-1078.

IAEA (2009). Research Reactor Modernization and Refurbishment, IAEA-TECDOC-1625, Vienna, Austria

IAEA (2008). Safety Analysis for Research Reactors. IAEA Safety Standards Series, No 55, IAEA, Vienna, Austria.

IAEA (2005). Safety of Research Reactors, Safety Requirements. IAEA Safety Standards Series, $\mathrm{N}^{\mathrm{o}}$ NS-R-4. IAEA, Vienna, Austria. 
Khan, L. A., Ahmad, N., Zafar, M. S., Ahmad, A. (2000). Reactor physics calculations and their experimental validation for conversion and upgrading of a typical swimming pool type research reactor. Vol. 27, Annals of Nuclear Energy, pp. $873-885$.

Khedr, A., Adorni, M., D'Auria, F. (2005). The effect of code user and boundary conditions on RELAP calculations of MTR research reactor transient scenarios. Nuclear Technology \& Radiation Protection, Vol. 1, pp. 16-22.

Marcum, W. R., Woods, B. G., Reese, S. R. (2010). Experimental and theoretical comparison of fuel temperature and bulk coolant characteristics in the Oregon State TRIGA ${ }^{\circledR}$ reactor during steady state operation. Nuclear Engineering and Design, Vol. 240, pp. 151-159.

Mesquita, A. Z., Rezende, H. C., Souza, R. M. G. P., 2009. Thermal power calibrations of the IPR-R1 TRIGA nuclear reactor. Proceedings of the 20th International Congress of Mechanical Engineering, COBEM 2009, November 15-20, Gramado, Brazil.

NEA - Nuclear Energy Agency (2009). Nuclear Fuel Behaviour in Loss-of-coolant Accident (LOCA) Conditions, State-of-the-art Report, ISBN 978-92-64-99091-3, OECD 2009.

Papin, J., Petit, M., Grandjean, C., Georgenthum, V. (2006). IRSN R\&D studies on high burnup fuel behaviour under RIA and LOCA conditions. Proceedings of Top Fuel 2006, pp. 274-278, Salamanca, Spain, October 22-26, 2006.

Petruzzi, A. and D'Auria, F. (2008) Thermal-hydraulic system codes in nuclear reactor safety and qualification procedures. Science and Technology of Nuclear Installations, Vol. 2008, doi:10.1155/2008/460795, pp. 1-16.

Reis, P. A. L., Costa, A. L., Pereira, C., Silva, C. A. M., Veloso, M. A. F., Mesquita, A. Z. (2011). Sensitivity analysis of the RELAP5 nodalization to IPR-R1 TRIGA research reactor, In: International Conference on Mathematics and Computational Methods Applied to Nuclear Science and Engineering (MEC 2011), Rio de Janeiro, Brazil, May 8-12, 2011, ISBN 978-85-63688-00-2.

Reis, P. A. L., Costa, A. L., Pereira, C., Veloso, M. A. F., Mesquita, A. Z., Soares, H. V., Barros, G. P., (2010). Assessment of a RELAP5 model for the IPR-R1 TRIGA research reactor. Annals of Nuclear Energy, Vol. 37, pp. 1341-1350.

Shoushtari, M. K., Kakavand, T., Ghaforian, H., Sadat Kiai, S. M. (2009). Preliminary scoping study of some neutronic aspects of new shim safety rods for a typical 5MW research reactor by Monte Carlo simulation. Nuclear Engineering and Design, Vol. 239, pp. 239-243.

Stamatelatos, I. E., Varvayanni, M., Tzika, F., Ale, A. B. F. Catsaros, N. (2007). Monte Carlo simulation of the Greek Research Reactor neutron irradiation facilities. Nuclear Instruments and Methods in Physics Research, Vol. 263, pp. 136-139.

Terremoto, L. A. A., Zeituni, C. A., Perrotta, J. A., da Silva J. E. R. (2000). Gamma-ray spectroscopy on irradiated MTR fuel elements. Nuclear Instruments and Methods in Physics Research A, Vol. 450, pp. 495-514.

Velit, C. G. and Primm, R. T. (2008). Partial safety analysis for a reduced uranium enrichment core for the high flux isotope reactor, Joint International Workshop: Nuclear Technology and Society - Needs for Next Generation, pp. 1-6, Berkeley, California, January 6-8, 2008.

Verfondern, K., Nabielek, H., Kendall, J. M. (2007). Coated particle fuel for high temperature gas cooled reactors. Nuclear Engineering and Technology, Vol. 39, pp. 603 - 616.

Woodruff, W. L., Hanan, N. A., Smith, R. S., Matos, J. E. (1996). A Comparison of the PARET/ANL and RELAP5/MOD3 codes for the analysis of IAEA Benchmark transients, Proceedings of the International Meeting on Reduced Enrichment for Research and Test Reactors, pp. 1-11, Seoul, Republic of Korea, October 7-10, 1996. 


\title{
Development of an Appendix K Version of RELAP5-3D and Associated Deterministic-Realistic Hybrid Methodology for LOCA Licensing Analysis
}

\author{
Thomas K. S. Liang \\ Shanghai Jiao Tong University \\ China
}

\section{Introduction}

The Loss of Coolant Accident (LOCA) is one of the most important design basis accidents (DBA). In light water reactors, particularly the pressurized water reactor (PWR), the severity of a LOCA will limit how high the reactor power can operate. In the regulatory analysis (USNRC, 1987), it was estimated that if the peak cladding temperature (PCT) during a LOCA decreases by $100^{\circ} \mathrm{F}$, it would be possible to raise the plant power by $10 \%$. The revision of 10 CFR50.46 in 1988 stated that two kinds of LOCA licensing approaches can be accepted, namely the realistic and Appendix $\mathrm{K}$ methodologies. The realistic licensing technique describes the behavior of the reactor system during a LOCA with best estimate (BE) codes. However, the uncertainties of BELOCA analysis must be identified and assessed so that the uncertainties in the calculated results can be estimated to a high confidence level. Alternatively, the Appendix $\mathrm{K}$ approach will guarantee the conservatism of the calculation results, instead of answering the analytical uncertainty. It is widely believed that the realistic approach can more precisely calculate the sequences of a LOCA accident, and therefore provides a greater margin for the PCT evaluation. The associated margin can be more than 200K (Westinghouse, 2009). However, the development of a realistic LOCA methodology is long and costly, and the safety authority is highly demanding in their approach to evaluate uncertainties. Instead, implementation of evaluation models required by Appendix $\mathrm{K}$ of 10 CFR 50 (USNRC, 1988) upon an advanced thermal-hydraulic platform, such as RELAP5-3D (RELAP5-3D Code development Team, 1998), TRAC (Liles et al., 1981), CATHARE (Bestion, 1990) et al., also can gain significant margin in the PCT calculation. For instance, the PCT of Taiwan's Maanshan Nuclear Power Plant calculated by the latest Westinghouse Appendix K Evaluation Model BASH (Westinghouse, 1987) is $445^{\circ} \mathrm{F}\left(2170^{\circ} \mathrm{F} \rightarrow 1725^{\circ} \mathrm{F}\right)$ lower than that of 1981's calculation (Taipower Company, 1982).

To develop a new Appendix K LOCA licensing tool using the most advanced version of RELAP5, namely RELAP5-3D, the compliance of the advanced RELAP5-3D code with Appendix $\mathrm{K}$ of 10 CFR 50 has been evaluated, and it was found that there are nine areas where code assessment and/or further modifications were required to satisfy the requirements set forth in Appendix K of 10 CFR 50. All of the ten areas have been evaluated 
and the RELAP5-3D has been successfully modified to fulfill the associated requirements. It was also demonstrated that all the phases of both LBLOCA and SBLOCA can be covered in RELAP5-3D/K.

To quantify uncertainty in BELOCA analysis, generally there are two categories of uncertainties required to be identified and quantified, which involve model uncertainties and plant status uncertainties. Particularly, it will take huge effort to systematically quantify individual model uncertainty of a best estimate LOCA code. Instead of applying a full ranged BELOCA methodology to cover both model and plant status uncertainties, a deterministicrealistic hybrid methodology (DRHM) was also developed to support LOCA licensing analysis with RELAP5-3D/K. Regarding the DRHM methodology, Appendix K deterministic evaluation models are adopted to ensure model conservatism, while CSAU methodology (Boyack, B., et al., 1989) is applied to quantify the effect of plant status uncertainty on PCT calculation. Generally, DRHM methodology can generate about 80-100K (Liang, et al., 2011) margin on PCT as compared to traditional Appendix K bounding state LOCA analysis.

\section{Development and assessment of RELAP5-3D/K}

To develop an Appendix K version of RELAP5-3D, the best-estimate version of RELAP5-3D was modified and assessed (Liang et al., 2002) to fulfill requirements set forth in Appendix K of 10CFR50. Nine build-in models in RELAP5-3D need to be modified and assessed (Schultz et al., 1999), which include (1) Metal-Water Reaction Rate; (2) Discharge Model; (3) ECC Bypass Model; (4) Critical Heat Flux During Blowdown; (5) Post-CHF Heat Transfer During Blowdown; (6) Prevention from Returning to Nucleate Boiling and Transition Boiling Heat Transfer Prior to Reflood; (7) Core Flow Distribution During Blowdown; (8) Reflood rate for PWR; and (9) Refill and Reflood Heat Transfer for PWRs. Separate-effects experiments were applied to assess specific code models and ensure that each modification can function properly. The separate effects assessment cases for each modification are summarized in Table 1.

\begin{tabular}{|c|c|c|c|}
\hline No. & Case & Phenom enon/Model & $\begin{array}{c}\text { Applicable Appendix K } \\
\text { Section }\end{array}$ \\
\hline 1 & Cathcart oxidation data & Metal-water reaction & I.A. 5 \\
\hline 2 & Marviken Test 22 & Critic al flow & I.C.1.ab \\
\hline 3 & 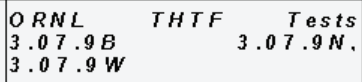 & Critic al he at flu $x$ & 1.C. 4 \\
\hline 4 & $\begin{array}{lrr}\text { ORNL THTF } & \text { Tests } \\
3.07 .9 \mathrm{~B} & & 3.07 .9 \mathrm{~N} \\
3.07 .9 \mathrm{~W} & & \end{array}$ & Film bo iling & I.C. 5 \\
\hline 5 & $\begin{array}{l}\text { ORNL THTF Test } \\
3.03 .6 \text { AR }\end{array}$ & Transition Boiling & I.C. 5 \\
\hline 6 & UPTF Test 6 & End of bypass & I.C.1.C \\
\hline 7 & $\begin{array}{l}\text { EPRI flow blockage } R u n 4 \\
\text { and } R \text { un } 8\end{array}$ & $\begin{array}{l}\text { Core blockaae and cross } \\
\text { flow }\end{array}$ & 1.C.7.a \\
\hline 8 & $\begin{array}{l}\text { FLECHT-SEASET, Tests } \\
31504, \quad 31203, \quad \text { and } \\
33338\end{array}$ & Refill and Reflood rates & I.D. 3 \\
\hline 9 & $\begin{array}{l}\text { FLECHT-SEASET } \\
31504, \quad 31203, \\
33338\end{array}$ & $\begin{array}{l}\text { Refilled and Reflond heat } \\
\text { transfer }\end{array}$ & 1.0 .5 \\
\hline
\end{tabular}

Table 1. Matrix of Separate-effect assessments 


\subsection{Code modification and Separate-effect assessments \\ 2.1.1 Metal-water reaction rate}

Since melting of fuel cladding is not the applicable domain, the parabolic rate low from the Baker-Just model (Baker et al., 1962) would be applied to calculate the fuel oxidation from zirconium-water reaction. Once the oxidation thickness has been evaluated, the associated amount of reaction heat added to the cladding and hydrogen generation also would be calculated. The Cathcart data (Cathcart, 1977) was used to assess the implementation of the Baker-Just models into RELAP5-3D. Cathcart measured the isothermal reaction rates of Zircaloy-4 tubes in steam at elevated temperatures. After the specified oxidation time, the tube was removed and the oxide thickness was measured using standard metallographic techniques. Typical assessment calculation is shown in Figure 1. It can be seen that at a higher bath temperature $\left(1500^{\circ} \mathrm{C}\right)$, the conservatism of the Baker-Just model is very clear.

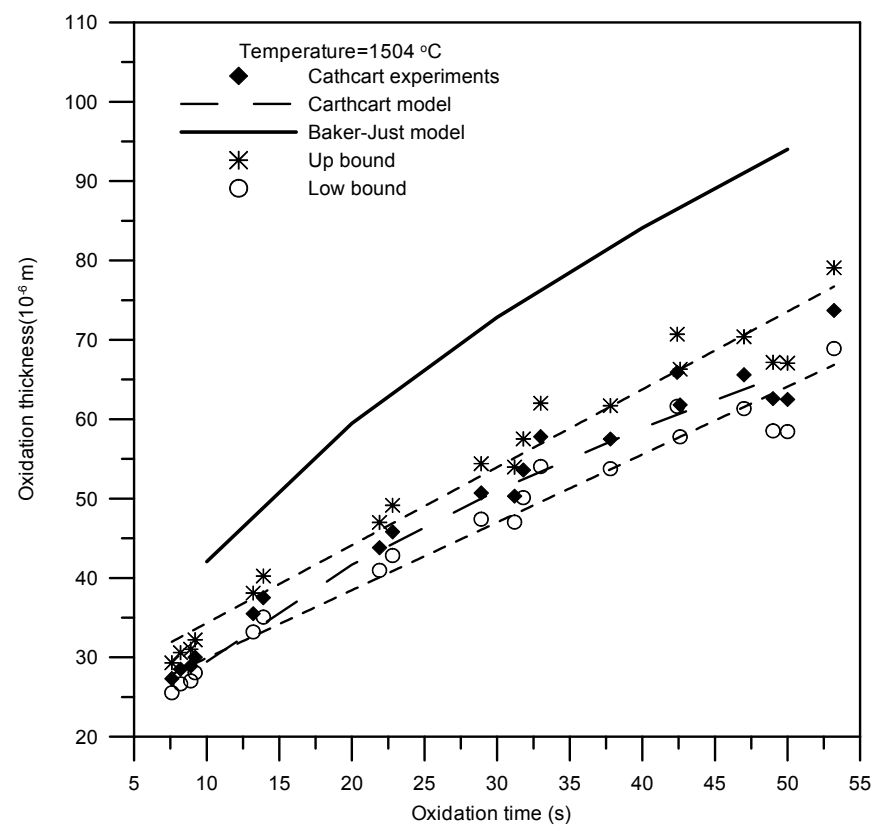

Fig. 1. Oxidation thickness of zirconium 4 (temperature $1504^{\circ} \mathrm{C}$ )

\subsubsection{Discharge model}

The Moody model (Moody, 1965) for the calculation of two phase choked flow and the Henry Fauske model (Fauske et al., 1971)for the single phase liquid choked flow were added to RELAP5-3D to make a break flow evaluation model. Regarding applying the Moody model, the stagnation conditions $\left(\mathrm{p}_{\mathrm{o}}, \mathrm{h}_{\mathrm{o}}\right)$ need to be derived from the cell center immediately upstream of the exit plane. The stagnation enthalpy can be calculated from the cell center properties as:

$$
h_{0}=\left(h_{f}+\frac{v_{f}^{2}}{2}\right)(1-x)+\left(h_{g}+\frac{v_{g}^{2}}{2}\right) x
$$


where the local enthalpies, fluid velocities and flow quality are evaluated at the equilibrium condition at the cell center. By assuming an isentropic process, the stagnation pressure can then be obtained from the local entropy defined by the cell center properties and the stagnation enthalpy through steam table iteration:

$$
P_{o}=P_{o}\left(h_{o}, s(h, P)\right)
$$

Data from Marviken Test 22 (Erickson et al., 1977) was used to assess the implementation of the Moody model. Marviken Test 22 was a full-scale critical flow test. The break was connected to the bottom of a large pressure vessel. The pressure vessel, which was originally part of the Marviken Nuclear Power Station in Sweden, was 5.2 meters in diameter and 24.6 meters tall. The vessel initially contained regions of subcooled liquid, saturated liquid and a steam dome. The assessment calculations against measured break flow are shown in Figure 2. The conservatism of the Moody model in two-phase choked flow was demonstrated.

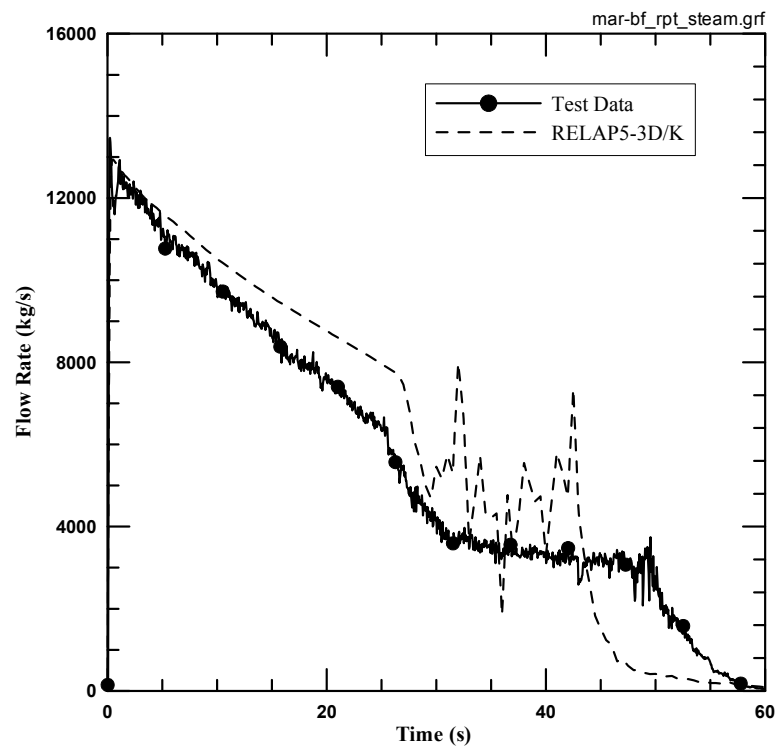

Fig. 2. Comparison of measured and calculated break flow

\subsubsection{ECC bypass model}

During the ECC bypass period, the emergency coolant would be held in the upper downcomer region. Those ECC water would accumulate in the inlet lines, and then leave RCS through the break without taking decay heat from the reactor core, until the vapor flow from the core can no longer sustain the water in the downcomer. The downcomer flooding model derived from the UPTF full-scale test (Siemens, 1988) was applied to determine when the ECC water could penetrate the downcomer through the RELAP5-3D regular CCFL input process. The UPTF downcomer flooding model is:

$$
j_{g}^{* 1 / 2}+2.193 j_{f}^{* 1 / 2}=0.6208
$$


According to the requirement, before the end of the bypass period all the injected ECC water needs to be removed from the system. To fulfill the ECC subtraction requirement, a set of time dependent junction and volume (TMDPJUN, TMDPVOL) would be connected to the cold leg of the broken loop close to the downcomer. Equal amount of injected ECC water will be forced to be on-line removed from the reactor system by this artificial set of TMDPJUN and TMDPVOL before the end of ECC bypass. The boron transport calculation of RELAP5-3D can indicate when the end of ECC bypass takes place. This boron model will trace the transport of the borated ECC water. Once the borated ECC water penetrates the downcomer and reaches the lower plenum, a signal of the end of ECC bypass will be generated and the ECC subtraction scheme via the TMDPJUN and TMDPVOL will be automatically terminated. The comparison of actual injected ECC water in the LOFT L2-5 (Davis, 1998) and the one calculated by the Appendix K model is shown in Figure 3.

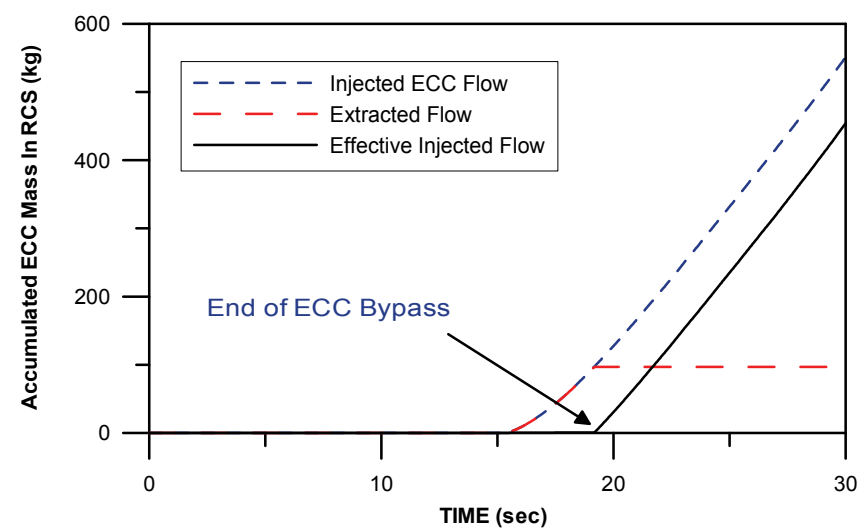

Fig. 3. Comparison of measured and calculated ECC water

\subsubsection{Critical heat flux during blowdown}

The set of three Appendix K CHF correlations used in RELAP4/MOD7 (Behling et al., 1981) would be adopted, which includes B\&W-2, Barnett and Hughes (modified Barnett) correlations, to cover the pressure range of interest. For the high-pressure range $(\mathrm{P}>10.34$ $\mathrm{MPa}), \mathrm{B} \& \mathrm{~W}-2$ was applied; for the medium pressure range $(8.96 \mathrm{MPa}>\mathrm{P}>6.89 \mathrm{MPa})$, Barnett correlation was applied; for the low-pressure range $(\mathrm{P}<5 \mathrm{MPa})$, the modified Branett was adopted. For pressures between ranges, interpolation by pressure was applied to calculate the correspond CHF:

$$
q_{C H F}=\frac{\left(P_{H}-P\right) q_{C_{H F}}+\left(P-P_{L}\right) q_{C H F_{H}}}{P_{H}-P_{L}}
$$

where index $\mathrm{H}$ and $\mathrm{L}$ represent the high and low ends of the interpolation range. Rod bundle heat transfer tests (Yoder et al., 1982) performed in the Thermal-Hydraulic Test Facility (THTF) at Oak Ridge National Laboratory (ORNL) were used to assess the CHF model and film boiling heat transfer. These tests were performed using an $8 \times 8$ fuel bundle. The rod geometry was representative of $17 \times 17$ fuel bundles, and the full-length bundle was electrically heated and had uniform axial and radial profiles. Three tests were used for 
assessment the $\mathrm{CHF}$ calculation, which include tests $3.07 .9 \mathrm{~B}, 3.07 .9 \mathrm{~N}$ and $3.07 .9 \mathrm{~W}$. The range of conditions during this test was representative of those expected during a large break LOCA. A typical comparison of the location first experiencing CHF is shown in Figure 4. It can be seen that the CHF location predicted by the EM models was conservatively lower.

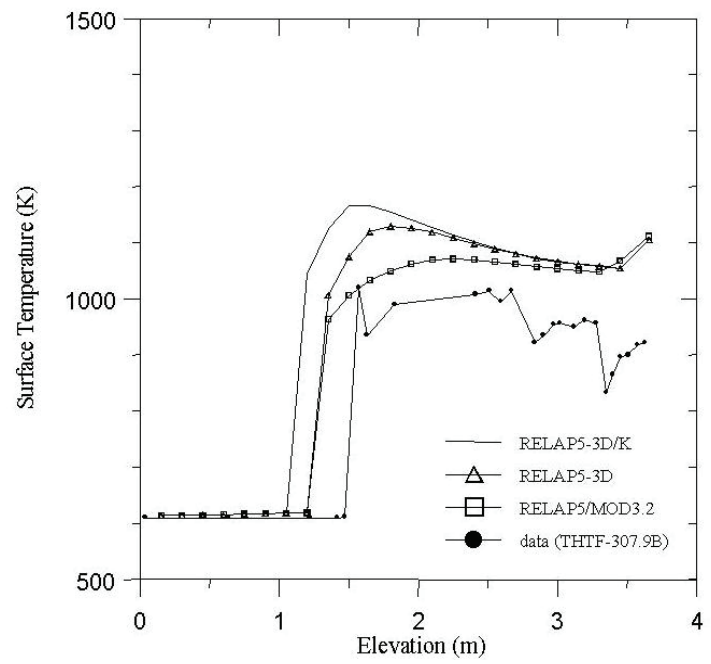

Fig. 4. Comparison of measured and calculated surface temperatures for THTF-307.9B test

\subsubsection{Post-CHF heat transfer during blowdown}

Two correlations suggested by Appendix K of 10 CFR 50 were adopted to calculate film boiling and transition boiling heat transfer. For the stable film boiling, Groeneveld 5.7 was applied, while the McDonough-Milich-King correlation was used for transition boiling heat transfer. Once CHF has occurred, the greater heat flux would be applied which were calculated by either the film boiling or transition boiling correlations. As stated in Appendix $\mathrm{K}$, the Groeneveld correlation shall not be used in the region near its low-pressure singularity. As suggested by INEEL (Schultz et al., 1999), for high flow $\left(j_{g}^{* 1 / 2}+j_{f}^{* 1 / 2}>1.36\right.$ for up flow, $j_{g}^{* 1 / 2}+j_{f}^{* 1 / 2}>3.5$ for downflow) if pressure is less than $1.38 \mathrm{MPa}$, the modified Dittus-Boelter correlation can be used to replace the Groeneveld correlation. If the core flow is not high, the modified Bromley correlation by Hsu with convection can be used to correct the low-pressure singularity. Typical assessments against THTF tests for film boiling heat transfer of the EM model are shown in Figure 5. As for the assessment of transition boiling heat transfer, THTF transition test with power ramping (THTF-303.6AR) was adopted. A typical comparison is shown in Figure 6.

\subsubsection{Prevention from returning to nucleate boiling and transition boiling}

As required by Appendix $\mathrm{K}$, during the blowdown phase once CHF occurs, transition boiling and nucleate boiling heat transfer shall not be reapplied for the remainder of the LOCA blowdown, unless the reflood phase of the transition has been entered. Assessment of the artificial prevention algorithm is shown in Figure 7. This figure depicts the mode 
change with and without the prevention algorithm. It can be seen that nucleate boiling heat transfer was successfully prevented by the algorithm which modifies the existing heat transfer logic.

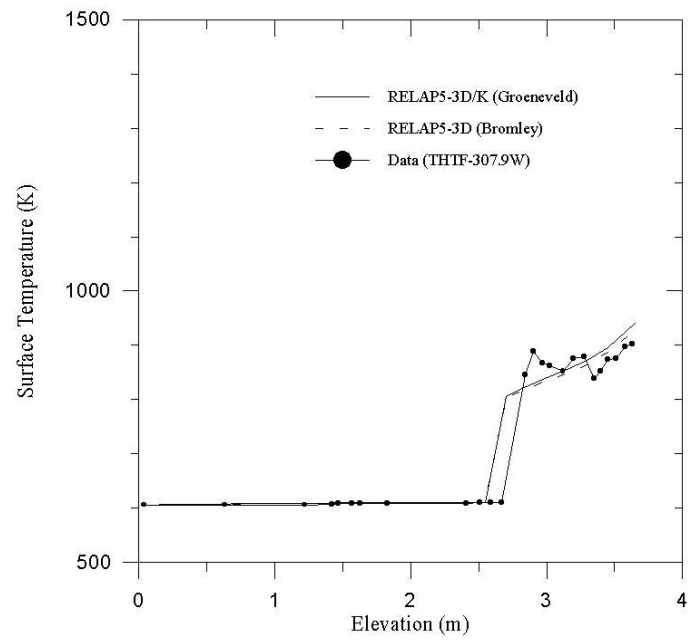

Fig. 5. Comparison of measured and calculated temperature changes for film boiling assessment

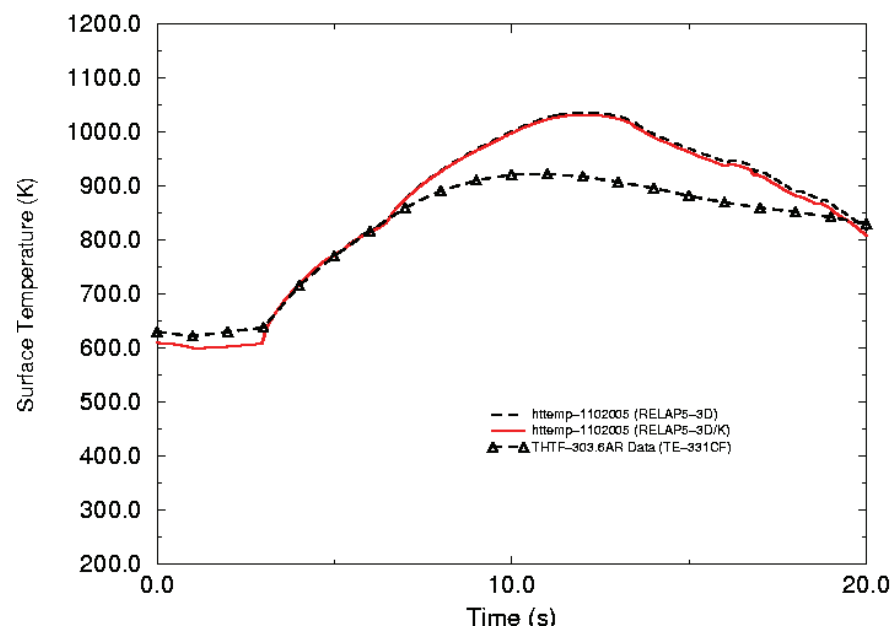

Fig. 6. Comparison of measured and for transition boiling assessment

\subsubsection{Core flow distribution during blowdown}

To fulfill the requirement of taking into account cross flow between regions and any flow blockage calculated to occur during blowdown as a result of cladding swelling or rupture, the feature of the cross flow junction of the RELAP5-3D would be applied. In cross flow 
junctions, the transverse momentum convection terms are neglected. Therefore, there is no transport of $\mathrm{x}$-direction momentum due to the flow in the transverse direction. To assess the calculation of core flow distribution under flow partial blockage, two EPRI flow blockage tests (Tapucu et al., 1984) were adopted in which single-phase liquid and two-phase air/water were used for a range of blockages and flow conditions. The comparisons of the calculated channel pressure distribution for the unblocked channel of the two-phase test against measurements is shown in Figure 8.

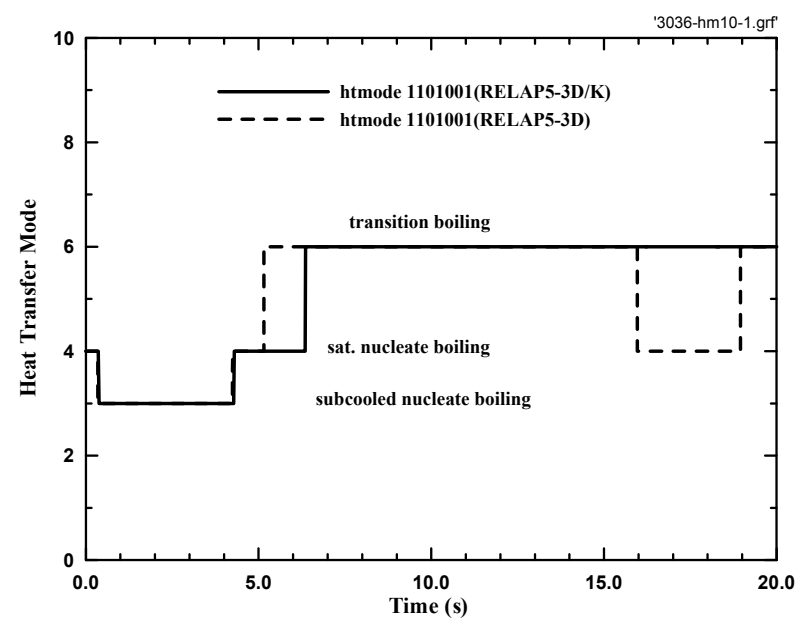

Fig. 7. Heat transfer mode calculated by the modified RELAP5-3D with \& w/o nucleate boiling lock-out

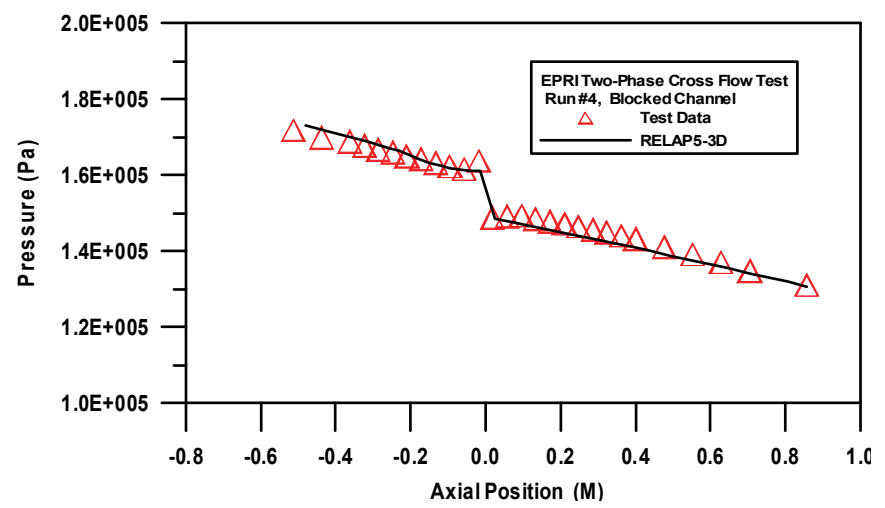

Fig. 8. Comparison of measured and calculated pressure distributions of the blocked channel

\subsubsection{Reflood rate for PWRs}

According to Appendix K of 10 CFR 50, the calculated carryover fraction and mass in bundle needs to be verified against applicable experimental data. In the existing PSI reflood 
model (Analytis, 1996) of RELAP5-3D, the modified Bestion correlation was used for interfacial drag in vertical bubbly-slug flow at pressures below 10 bars to replace the EPRI correlation. Above 20 bars the EPRI correlation was used. Between 10 and 20 bars the interfacial drag was interpolated. To assess the performance of the PSI model in the best estimate version of the RELAP5-3D, five FLECHT-SEASET tests $(31504,31203,31302,31805$ and 33338) (Loftus et al., 1980) were adopted. For the first four forced reflood tests, the flooding rates ranged from $0.81 \mathrm{inch} / \mathrm{s}$ to $3.01 \mathrm{inch} / \mathrm{s}$. As for the last gravity-driven reflood test, the flooding rate was up to $11.8 \mathrm{inch} / \mathrm{s}$ during the accumulator injection period. Typical assessments were shown in Figures 9 and 10. Through the assessments against five reflood tests, it was found that the PSI model could predict the flooding rate reasonable well but with enough conservatism.

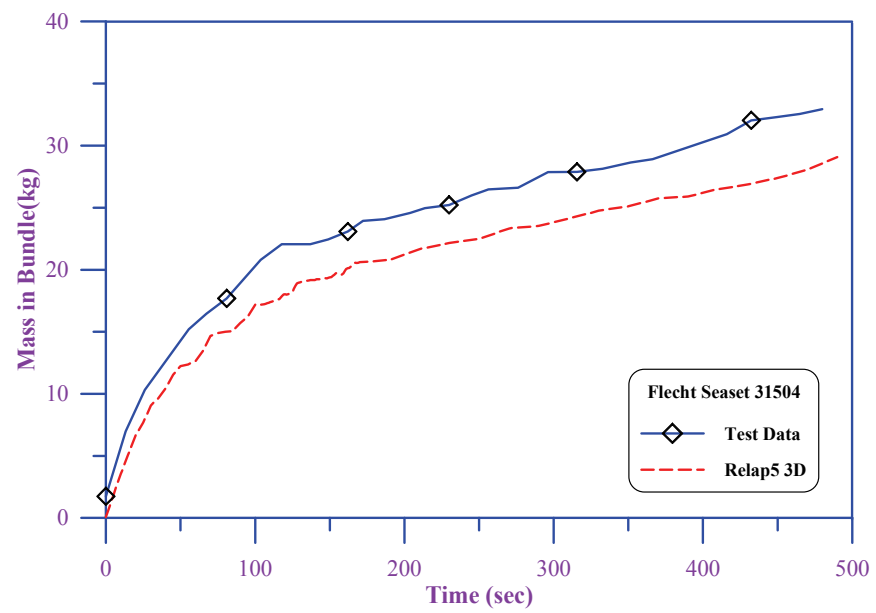

Fig. 9. Comparison of measured and calculated carryover fractions

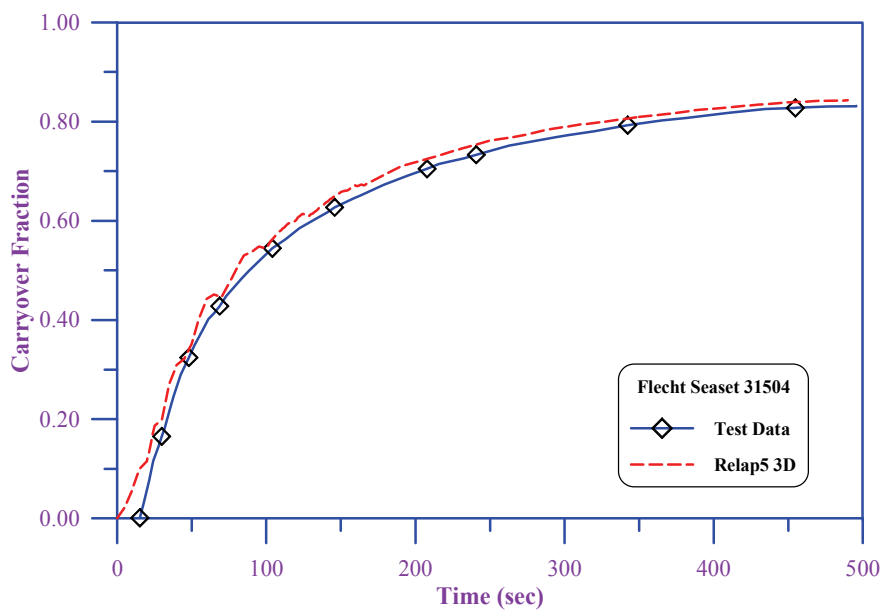

Fig. 10. Comparison of measured and calculated bundle masses 


\subsubsection{Refill and reflood heat transfer for PWRs}

During reflood phase, the RELAP5-3D PSI model was adopted to fulfill the Appendix K requirement for a flooding rate greater than $1 \mathrm{inch} / \mathrm{sec}$ with necessary modifications. In the PSI model, a modified Weisman correlation calculating the heat transfer to liquid and a modified Dittus-Boelter correlation calculating the heat transfer to vapor replace the Chen transition boiling correlation. As for film boiling, heat transfer to liquid uses the maximum of a film coefficient contributed by the modified Bromley correlation, and a ForslundRohsenow coefficient. In addition, radiation to droplets is added to the final film-boiling coefficient to liquid. The heat transfer to vapor for film boiling is the same as the one for transition boiling, which was calculated by the modified Dittus-Boelter. As required by the Appendix K of 10 CFR 50, when the flooding rate is less than 1 inch/s, only steam cooling in the PSI model was allowed. Assessment calculations were performed to against the five FLECHT SEASET tests discussed above. To bind the peak cladding temperature (PCT) span on each measured fuel rods at the same elevation, the calculated heat transfer coefficient calculated by the original PSI model was reduced by a factor of 0.6 for the flooding rate greater than $1 \mathrm{inch} / \mathrm{sec}$ to ensure reasonable conservatism. Typical comparison of the PCTs is shown in Figures 11. While the comparison of heat transfer coefficients is shown in Figures 12.

FLECHT SEASET Reflood Experiment:Test 31203

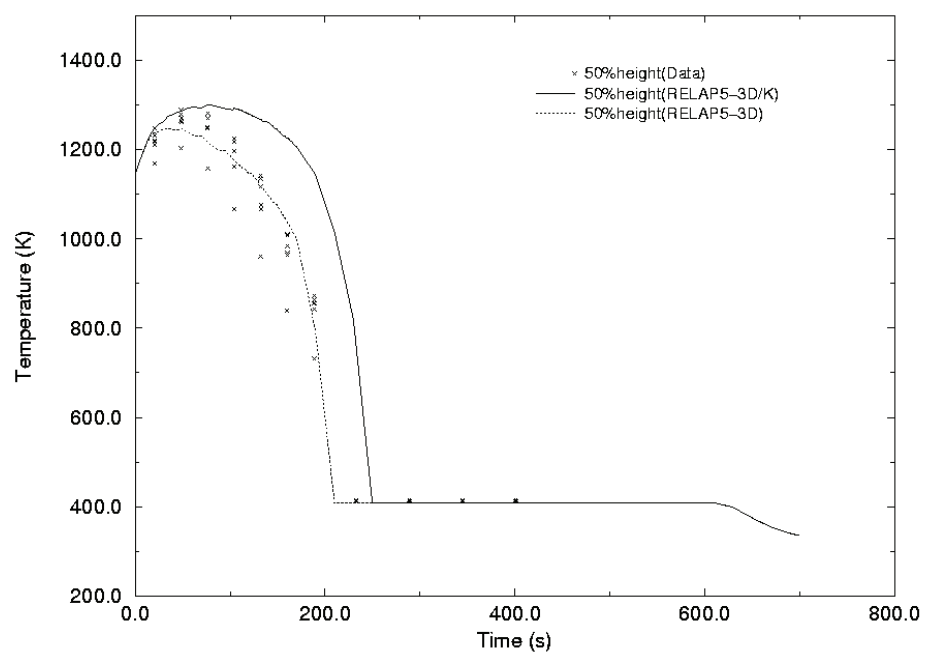

Fig. 11. Comparison of measured and calculated peak cladding temperatures

\subsection{RELAP5-3D/K integral-effect assessments}

To verify the overall conservatism of the newly developed Appendix K version of RELAP53D, 11 sets of integral LOCA experimental data covering SBLOCA and LBLOCA for both PWR and BWR, were applied, as listed in Table 2 and Table 3 for both PWR and BWR respectively. In this paper, only integral assessments LOFT LBLOCA experiment L2-5 (Anklam et al., 1982) and SBLOCA S-LH-1 (Grush et al., 1981) were summarized. 
FLECHT SEASET Reflood Experiment:Test 31203

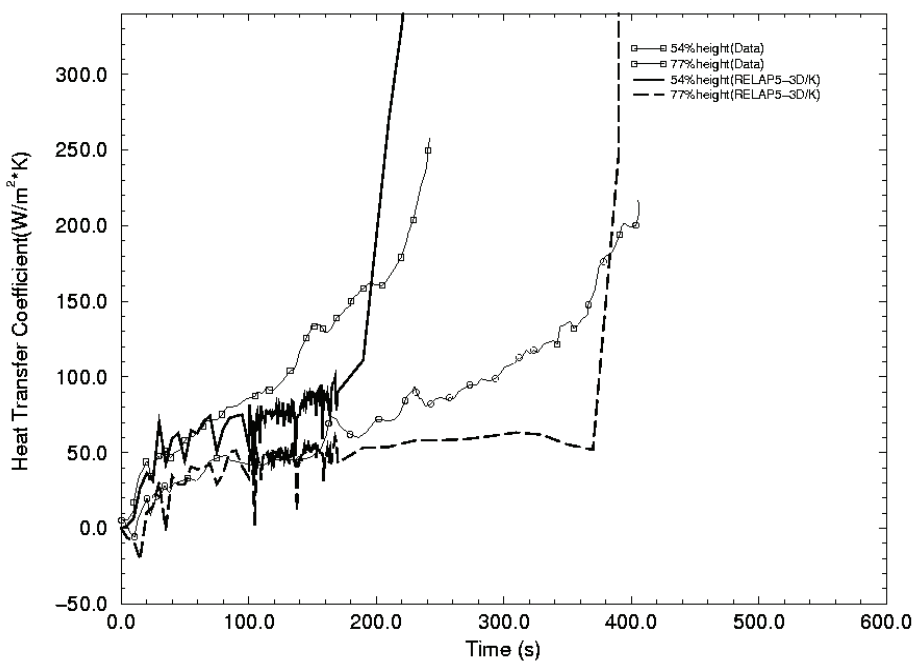

Fig. 12. Comparisons of measured and calculated heat transfer coefficients

\begin{tabular}{|c|c|c|c|c|c|c|c|}
\hline Cases & L2-3 & L2-5 & Lp-Lb-1 & S-06-3 & L3-7 & S-LH-1 & IIST \\
\hline Break Size & $200 \%$ & $200 \%$ & $200 \%$ & $200 \%$ & $0.1 \%$ & $5 \%$ & $2 \%$ \\
\hline $\begin{array}{c}\text { Break } \\
\text { Location }\end{array}$ & Cold Leg & Cold Leg & Cold Leg & Cold Leg & Cold Leg & Cold Leg & Cold Leg \\
\hline Notes & $\begin{array}{c}\text { RCP } \\
\text { Running }\end{array}$ & $\begin{array}{c}\text { RCP } \\
\text { Tripped }\end{array}$ & $\begin{array}{c}\text { Higher } \\
\text { Power }\end{array}$ & $\begin{array}{c}\text { RCP } \\
\text { Running }\end{array}$ & $\begin{array}{c}\text { Without } \\
\text { Core } \\
\text { Heatup }\end{array}$ & $\begin{array}{c}\text { With Core } \\
\text { Heatup }\end{array}$ & $\begin{array}{c}\text { With } \\
\text { Core } \\
\text { Heatup }\end{array}$ \\
\hline
\end{tabular}

Table 2. Matrix of PWR LOCA integral effect assessments

\begin{tabular}{|c|c|c|c|c|}
\hline Cases & TLTA 6425 & FIST 6DBA1B & FIST 6LB1A & FIST 6SB2C \\
\hline Break Size & $200 \%$ & $200 \%$ & $100 \%$ & $2 \%$ \\
\hline Break Location & Recir. Line Break & Recir. Line Break & LPCI Line Break & Recir. Line Break \\
\hline Notes & & & ADS Actuation & HPCS Unavailable \\
\hline
\end{tabular}

Table 3. Matrix of BWR LOCA integral effect assessments

\subsubsection{LBLOCA assessment}

In the assessment of LOFT L2-5, important parameters including break flow, downcomer water level and hot spot heat transfer coefficient calculated from both evaluation model (EM) and best estimate (BE) model were shown in Figures 13,14 and 15 respectively. It can be seen that results from EM model are relatively conservative. The comparison of peak cladding temperature (PCT) against measurement was shown in Figure 16. The calculated PCT from EM model clearly bounds not only the BE PCT but also all the measurement scatterings. The conservative PCT calculated by RELAP5-3D/K against LBLOCA experiments 
from both LOFT and Semi-scale was summarized in Table 4 and the conservative trend is shown in Figure 17. It can be seen that RELAP5-3D/K can conservatively predict PCT by 60$260 \mathrm{~K}$.

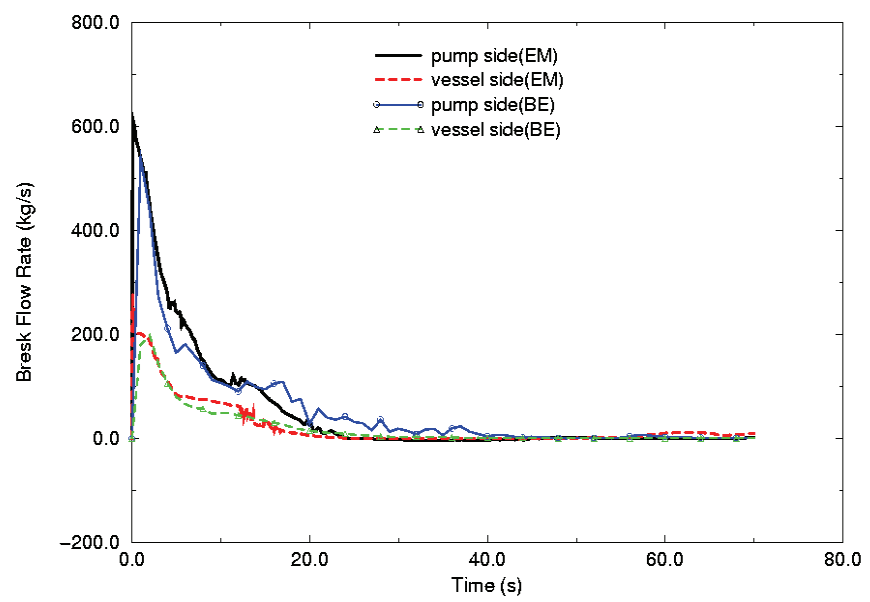

Fig. 13. Comparison of break flow of LOFT LBLOCA L2-5

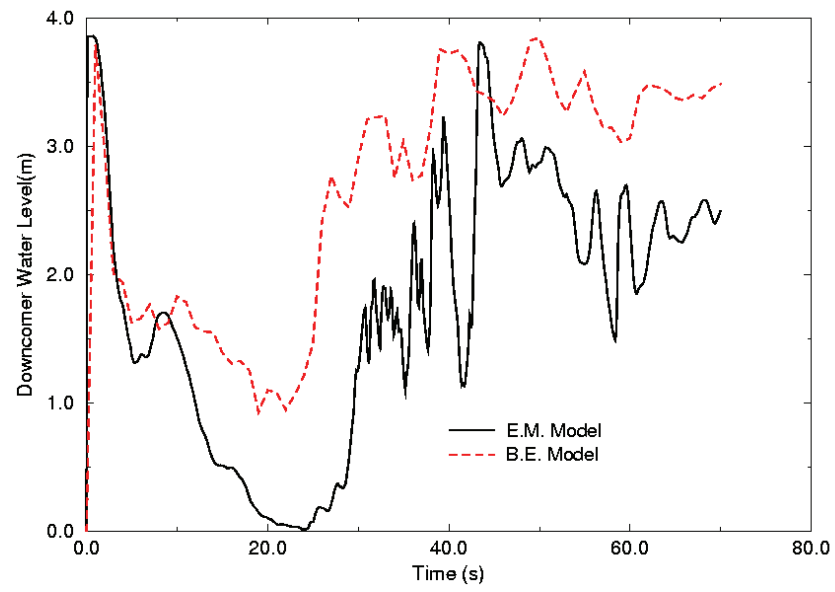

Fig. 14. Comparison of downcomer water level of LOFT LBLOCA L2-5

\subsubsection{SBLOCA assessment}

SBLOCA experiment Semi-Scale S-LH-1 is a typical 5\% cold break. Most important SBLOCA phenomena were involved in S-LH-1 experiment, which includes early core uncover caused by the core level depression, loop seal clearance and later core heat up caused by core boiled off. The calculated break flow, core water level and PCT against S-LH-1 (5\% SBLOCA) were shown in Figures 18, 19 and 20 respectively. The conservatism of RELAP5-3D/K in SBLOCA analysis generally can be observed. 


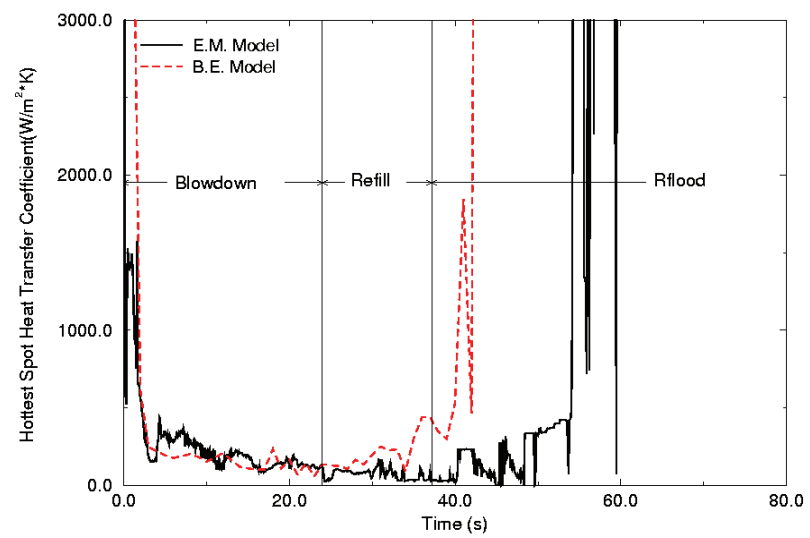

Fig. 15. Comparison of core heat transfer coefficient of LOFT LBLOCA L2-5

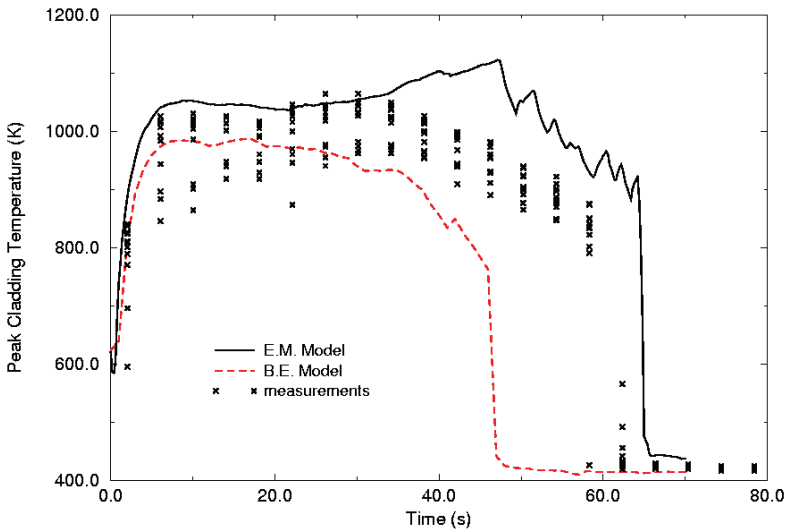

Fig. 16. Comparison of peak cladding temperature of LOFT LBLOCA L2-5

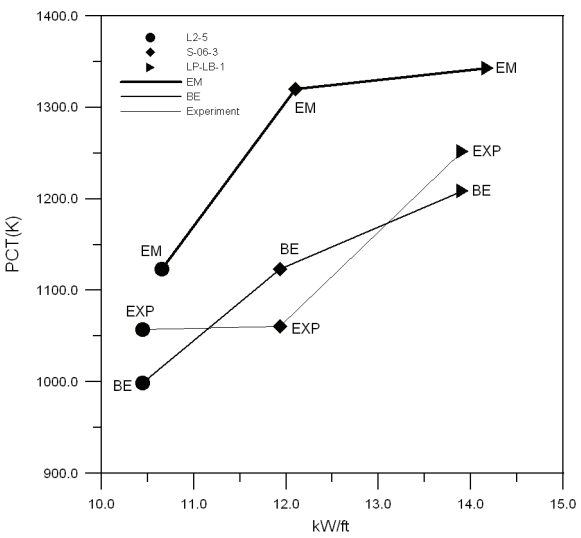

Fig. 17. Conservative trend of PCT calculated by RELAP5-3D/K 


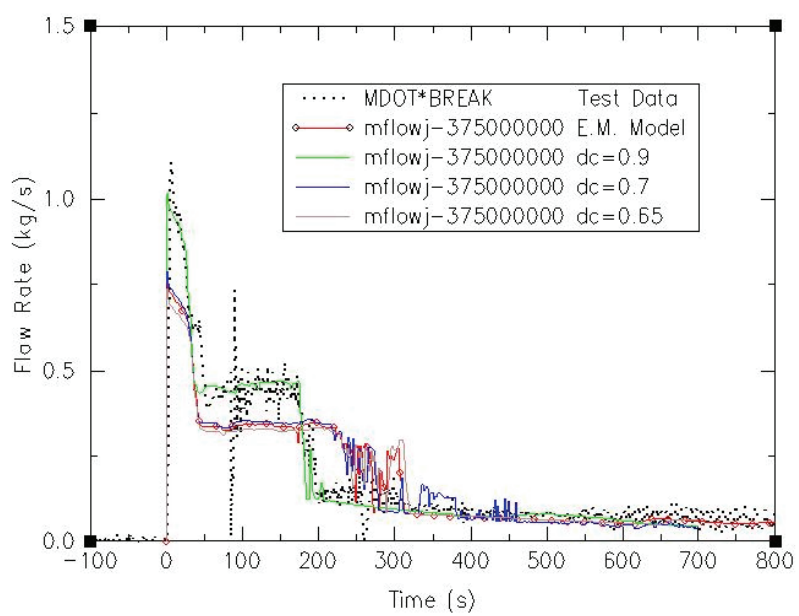

Fig. 18. Comparison of breaks flow of semiscale SBLOCA S-LH-1

\begin{tabular}{|c|c|c|c|c|}
\hline Cases & $\begin{array}{c}\text { Measured } \\
\mathrm{PCTs}\left({ }^{\circ} \mathrm{K}\right)\end{array}$ & $\begin{array}{c}\text { PCTs by BE } \\
\text { Model }\left({ }^{\circ} \mathrm{K}\right)\end{array}$ & $\begin{array}{c}\text { PCTs by EM } \\
\text { Model }\left({ }^{\circ} \mathrm{K}\right)\end{array}$ & $\begin{array}{c}\text { PCT }\left({ }^{\circ} \mathrm{K}\right) \\
\left(\mathrm{PCT}_{\text {EM }}-\mathrm{PCT}_{\text {exp }}\right)\end{array}$ \\
\hline L2-5 & 1057.2 & 998.6 & 1123.1 & 65.9 \\
\hline L2-3 & 898.3 & 938.1 & 1094.6 & 196.3 \\
\hline LP-LB-1 & 1252.4 & 1290.5 & 1343.4 & 91.0 \\
\hline S-06-3 & 1061.2 & 1123.7 & $1320.5\left(1271.2^{*}\right)$ & $259.3\left(210.0^{*}\right)$ \\
\hline TLTA6425 & 608.9 & 599.7 & 745.0 & 136.1 \\
\hline FIST 6DBA1B & 646.9 & 691.3 & 714.9 & 68.0 \\
\hline
\end{tabular}

Table 4. Summary of LBLOCA assessments

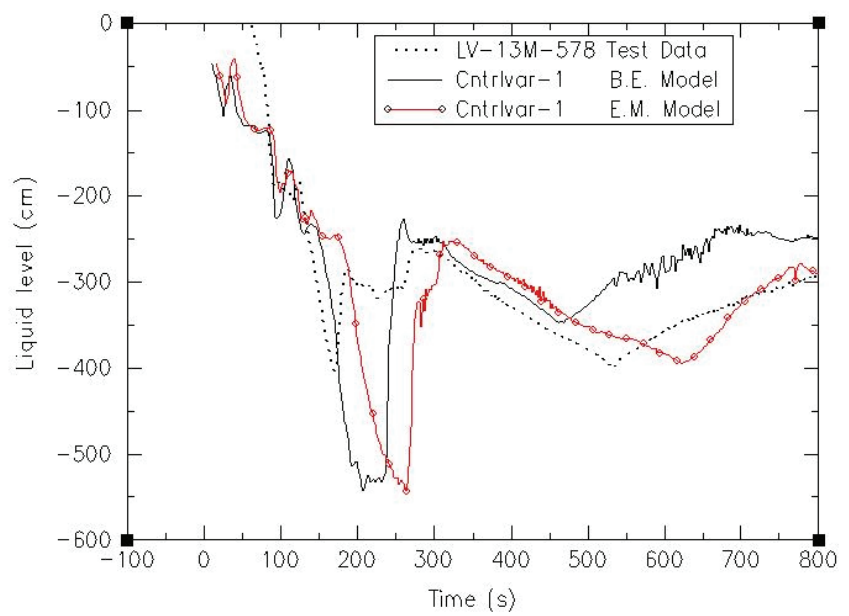

Fig. 19. Comparison of core water level of semiscale SBLOCA S-LH-1 


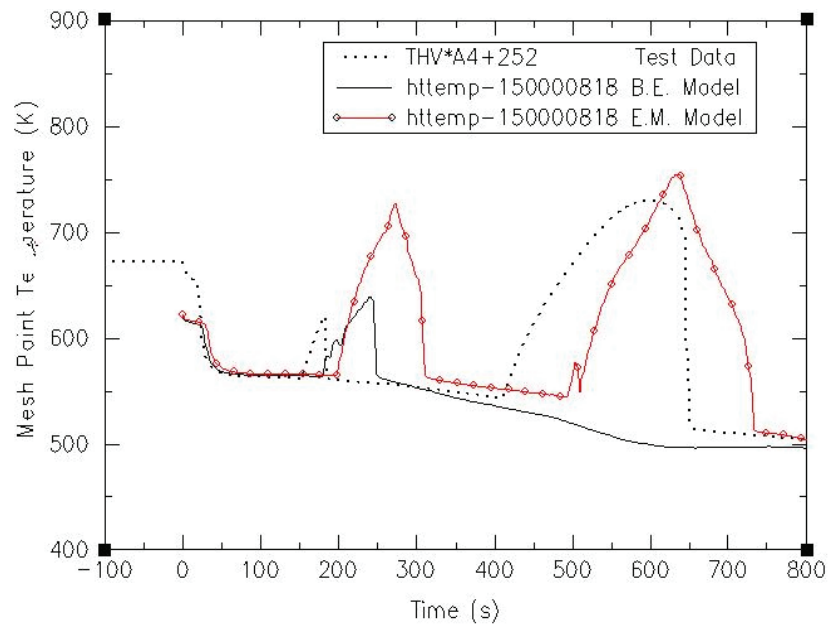

Fig. 20. Comparison of peak cladding temperature of semiscale SBLOCA S-LH-1

\section{Deterministic-realistic hybrid methodology for LOCA licensing analysis}

Instead of applying a full ranged BELOCA methodology to cover both model and plant status uncertainties, a deterministic-realistic hybrid methodology (DRHM) was developed to support LOCA licensing analysis with RELAP5-3D/K. In the DRHM methodology, Appendix K evaluation models are still adopted to ensure conservatism of physical model, while CSAU methodology is applied to quantify the effect of plant status uncertainty on PCT calculation. To ensure the model conservatism, not only physical model should satisfy requirements set forth in the Appendix K of 10 CFR 50, sensitivity studies also need to be performed to ensure a conservative plant modeling.

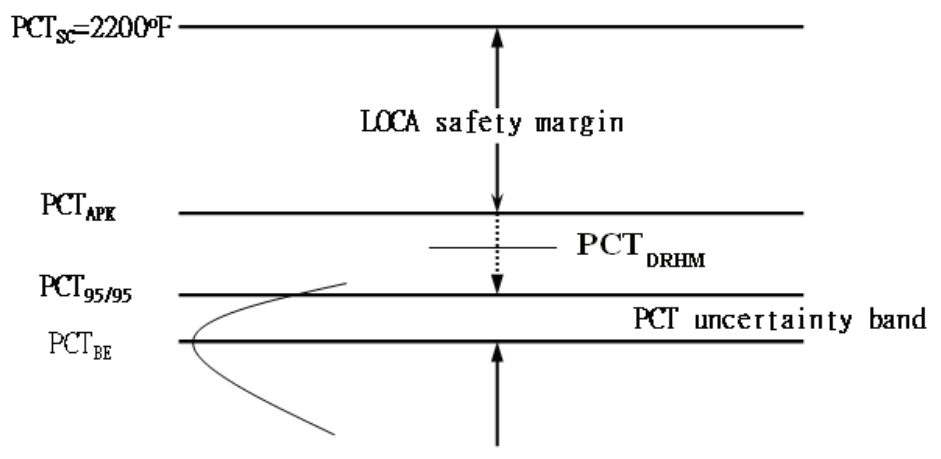

Limi ting LOCA events

IPCT

Fig. 21. PCT safety margins calculated by BE and appendix K LOCA methodologies 
To statistically consider the plant status uncertainties, which involve uncertainties of plant initial condition, accident boundary condition and plant system settings, the NRC endorsed CSAU methodology is applied. Three major elements are involved in the CSAU methodology, which are (I) requirements and capabilities, (II) assessment and ranging of parameters and (III) sensitivity and uncertainty analysis. Since Appendix K conservative models will be adopted to cover physical model uncertainties, model assessments stated in element II are not related. Instead, ranking and ranging of plant status uncertainty would be the major focus. The resulting PCT by using DRHM method theoretically can be lower than the $\mathrm{PCT}_{\mathrm{APK}}$ but higher

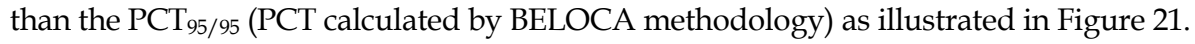

In DRHM methodology, six sequential steps are included, which are (1) ranking of plant status parameters, (2) ranging of plant status uncertainties, (3) development of a run matrix by random sampling, (4) using conservative E.M. model to perform LOCA analysis of each trial, (5) statistical analysis of calculated figure of merit (PCTs) and (6) determine licensing value of PCT. The procedure of DRHM is shown in Figure 22 and each step will be elaborated as following:

\begin{tabular}{|c|c|}
\hline Item Number & Uncertainty Attributes Plant Parameters \\
\hline 1 & Break Type \\
\hline 2 & Break Area \\
\hline 3 & Core Average Linear Heat Rate \\
\hline 4 & Initial Average Fluid Temperature \\
\hline 5 & Pressurizer Pressure \\
\hline 6 & Accumulator Liquid Volume \\
\hline 7 & Accumulator Pressure \\
\hline 8 & Accumulator Temperature \\
\hline 9 & Safety Injection Temperature \\
\hline 10 & Peak Heat Flux Hot Channel Factor (FQ) \\
\hline 11 & Peak Hot Rod Enthalpy Rise Hot Channel Factor (FDH) \\
\hline 12 & Axial Power Distribution (PBOT) \\
\hline 13 & Axial Power Distribution (PMID) \\
\hline 14 & Off-Site Power \\
\hline 15 & ECCS Capacity \\
\hline
\end{tabular}

Table 5. Major plant status parameters

\section{(1) Ranking of plant status parameters}

Plant parameters which will affect LOCA analysis can be generally divided into three groups, namely plant initial conditions, accident boundary conditions and plant system settings. Essential plant parameters need to be identified and ranked to limit the scope of uncertainty analysis. Typical PWR important plant status parameters are listed in Table 5. Major plant status parameters generally involve system initial conditions, core initial conditions, ECCS initial conditions, boundary conditions and system settings.

\section{(2) Ranging of plant status uncertainties}

To define the uncertainty of a plant parameter, not only the uncertainty range needs to be quantified, but also the distribution function needs to be specified. Three different kinds of 


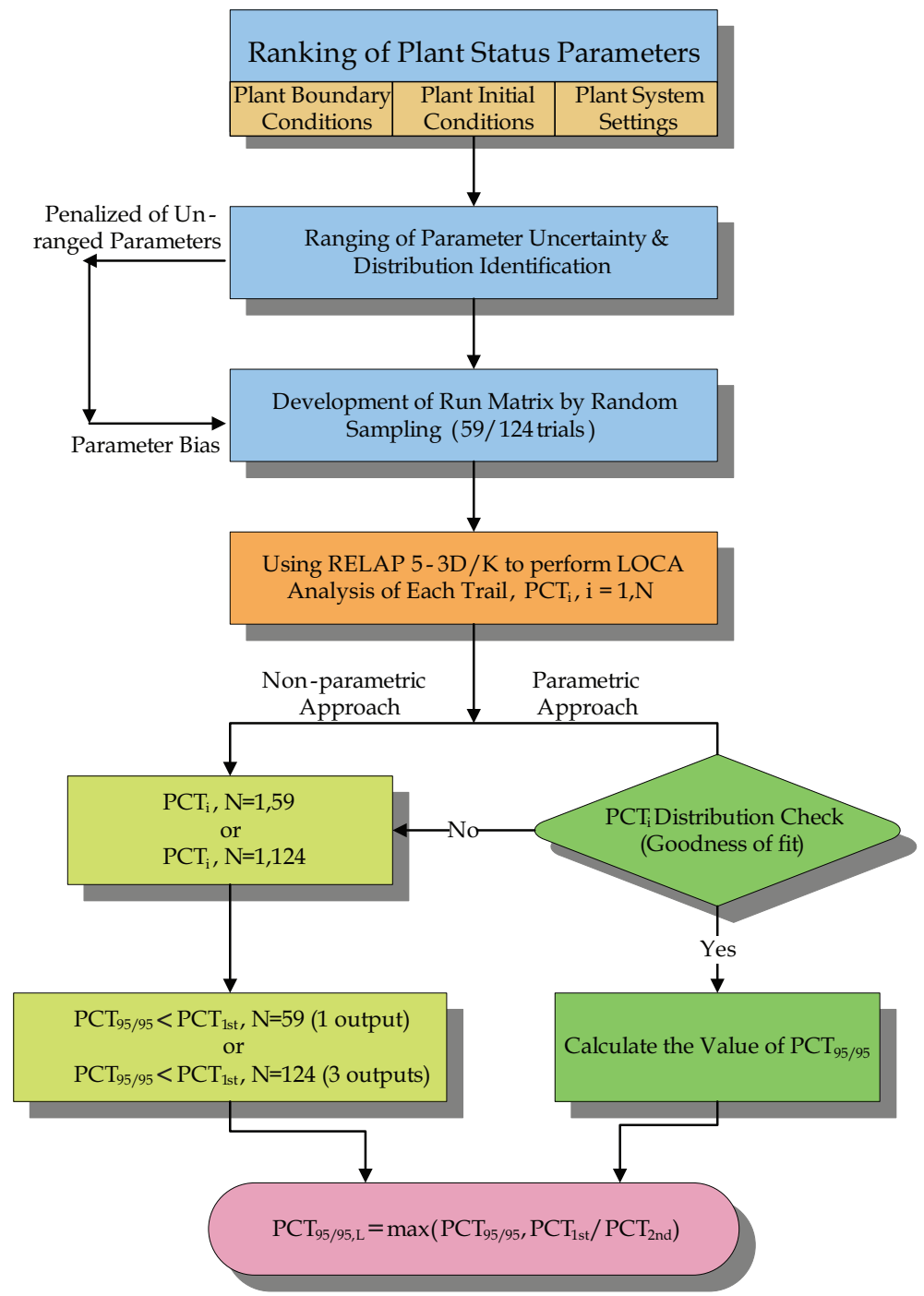

Fig. 22. Procedures of DRHM methodology

elements contribute the total uncertainty of a particular plant status parameter, which involve measurement uncertainty, fabrication uncertainty and normal operational range. For instance, the uncertainties of system pressure and coolant average temperature $\left(\mathrm{T}_{\text {avg }}\right)$ are majorly contributed by measurement uncertainty. While for the uncertainty of the total peaking factor $\left(\mathrm{F}_{\mathrm{Q}}\right)$, measurement uncertainty, fabrication uncertainty and operational uncertainty are all involved. The associated range of operational uncertainty of $F_{Q}$ can be determined by the nominal technical specification value (typically 2.274) and statistical upper bounding operating value (typically 2.000). As for the determination of power shape, the traditional bounding shape will be relaxed by sampling realistic operating shapes. Each 
operating power shape can be divided into three segments, $\mathrm{P}_{\text {mid }}, \mathrm{P}_{\text {bot }}$ and $\left(1-\mathrm{P}_{\text {mid }}-\mathrm{P}_{\text {bot }}\right)$. With the sampling values of $\mathrm{F}_{\Delta \mathrm{H}}, \mathrm{F}_{\mathrm{Q}}, \mathrm{P}_{\text {mid }}$ and $\mathrm{P}_{\text {bot, }}$ a unique power shape can be defined as shown in Figure 23.
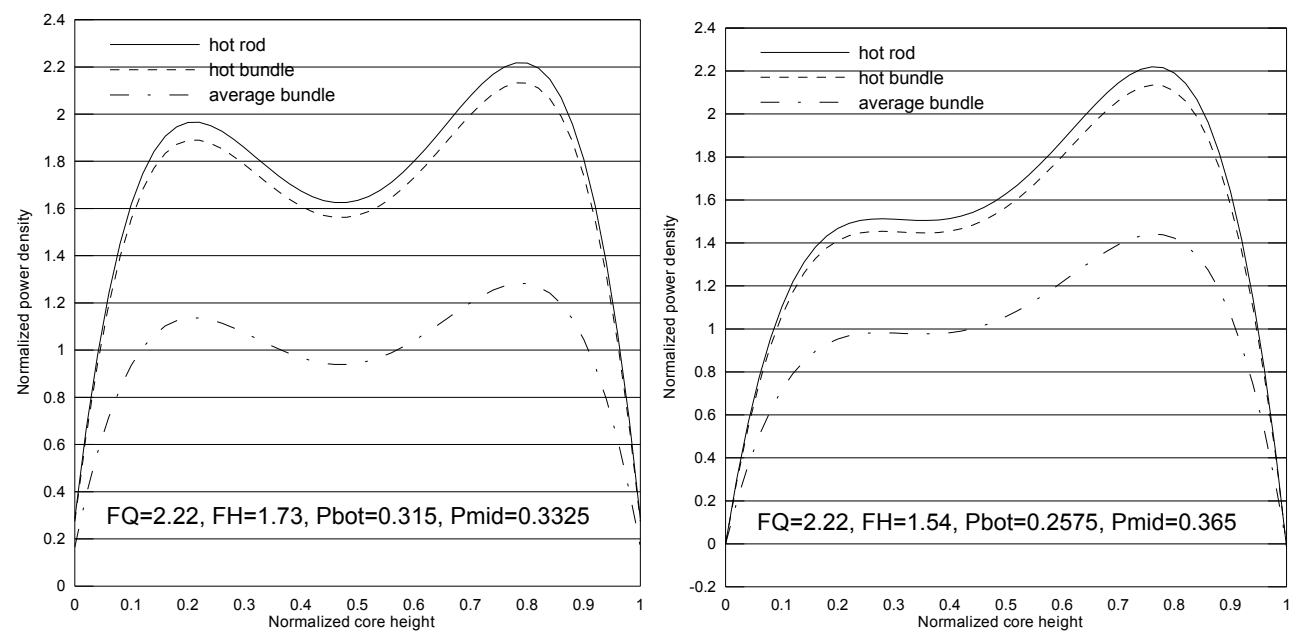

Fig. 23. Sampling of power shapes

\section{(3) Development of a run matrix by random sampling}

Once the major system parameters have been identified and ranged, random sampling of each parameter needs to be performed to generate a run matrix. Typical parameter samplings of $F_{Q}, P_{r c s}, T_{\text {avg }}$ and $P_{\text {acc }}$ are shown in Figure 24. The run matrix needs to consist of trials of 59 sets, 93 sets or 124 sets according to the order statistic method (David and Nagaraja, 1980).
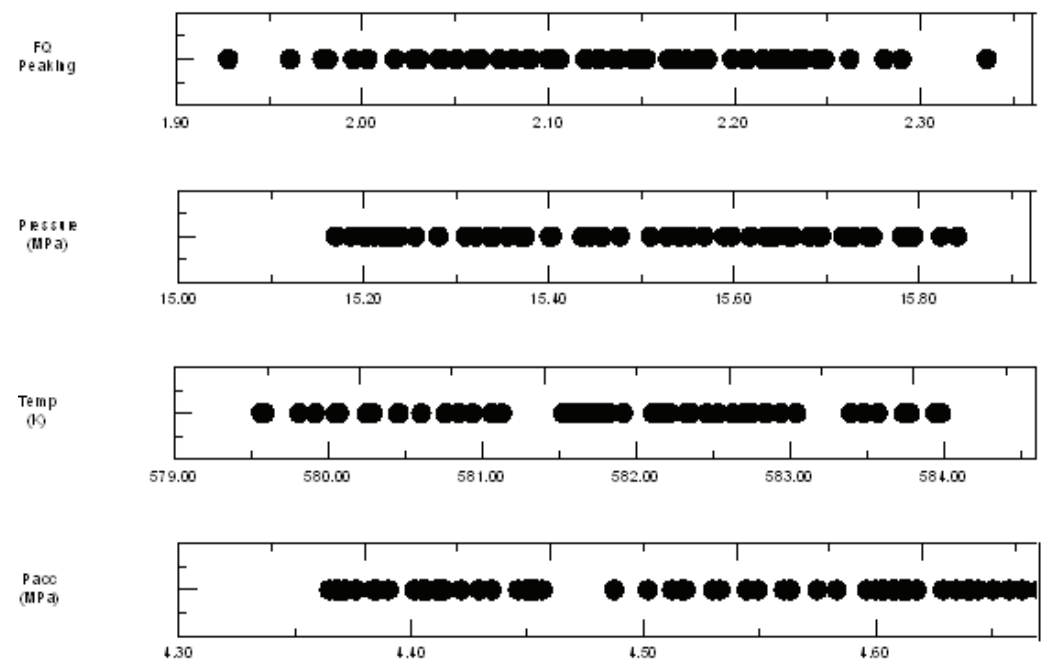

Fig. 24. Typical parameter sampling 


\section{(4) Using conservative plant E.M. model to perform LOCA analysis of each trial}

Conservative plant E.M. model will be applied to analyze each trial to calculate the PCT of each LOCA event. Regarding the conservative plant E.M. model, requirements of Appendix $\mathrm{K}$ for physical models will be satisfied, and a conservative plant specific model will be implemented based on sensitivity studies. Since RELAP5-3D/K is an Appendix K version of RELAP5-3D, it will be adopted to build a plant specific model.

\section{(5) Statistical analysis of calculated figure of merit (PCTs)}

Once the PCT of each trial can be calculated, both parametric (Devore, 2004) and nonparametric statistical approaches (David and Nagaraja, 1980) can be applied to determine the statistical upper tolerance limit. The parametric approach can directly calculate the $\mathrm{PCT}_{95 / 95}$ while the non-parametric approach can conservatively estimate of value of $\mathrm{PCT}_{95 / 95}$.

\section{Non-parametric approach}

In this approach, it is not necessary to identify the distribution of PCT outcomes. If only one outcome is cited from each trail, the Wilk's formula (David \& Nagaraja, 1980) can be applied to calculate the estimator the 95/95 upper tolerance limit.

$$
\beta=1-\gamma^{N}
$$

where $\beta$ is the confidence level, $\gamma$ is the tolerance interval and $N$ is the required number of samples. According to the Wilk's formula, the 95/95 value can be conservatively estimated by either the greatest PCT from 59 trials, the 2nd highest value of PCT from 93 trials or the 3rd highest value of PCT from 124 trials. That is:

$$
Y_{95 / 95} \approx Y_{1 s t}(59) \text { or } Y_{95 / 95} \approx Y_{2 n d}(93) \text { or } Y_{95 / 95} \approx Y_{3 r d}(124)
$$

If more than one outcome needs to be cited from each trial, the Guba's formula (Guba and Makai, 2003) can be used:

$$
\beta=\sum_{j=0}^{N-P} \frac{N !}{(N-j) ! j !} \gamma^{j}(1-\gamma)^{N-j}
$$

where $\mathrm{N}$ is the sample size and $\mathrm{P}$ is the number of output variables. If output variable is only one, the Guba formula will reduce to Wilk' formula.

\section{Parametric approach}

In this approach, the distribution of outcome needs to be identified by using fitting test, such as goodness-of-fitting test. If a certain distribution can be identified, such as normal distribution or uniform distribution, the population mean $\left(\mu_{\mathrm{p}}\right)$ and population standard deviation $\left(\sigma_{\mathrm{p}}\right)$ can be projected by sample mean $\left(\mu_{\mathrm{s}}\right)$ and sample standard deviation $\left(\sigma_{\mathrm{s}}\right)$ under a certain confidence level, such as $95 \%$. The sample mean $\left(\mu_{\mathrm{s}}\right)$ and sample standard deviation $\left(\sigma_{\mathrm{s}}\right)$ are:

$$
\mu_{s}=\sum_{i=1}^{n} x_{i} / n, \quad \sigma_{s}=\sqrt{\left[\frac{\sum_{i}^{n} x_{i}^{2}}{n-1}-\left(\frac{n}{n-1}\right) * \mu_{s}^{2}\right]}
$$


If normal distribution can be assumed by goodness-of-fitting test, the $\mu_{\mathrm{p}}$ and $\sigma_{\mathrm{p}}$ under a given confidence level can be expressed as:

$$
\begin{gathered}
\mu_{p} \leq\left[\mu_{s}+t_{\alpha}(n-1) * \sigma_{s} / \sqrt{n}\right] \\
\sigma_{p}^{2} \leq \frac{\sigma_{s}^{2}(n-1)}{\chi_{1-\alpha}^{2}(n-1)}
\end{gathered}
$$

where $t_{\alpha}(n-1)$ is the student $t$ variable at (1- $\left.\alpha\right)$ confidence level under $(n-1)$ degree of freedom, $\chi_{1-\alpha}^{2}(n-1)$ is $\chi^{2}$ variable at $(1-\alpha)$ confidence level under $(n-1)$ degree of freedom. Once $\mu_{\mathrm{p}}$ and $\sigma_{\mathrm{p}}$ are projected at $95 \%$ confidence level $\left(\mu_{\mathrm{p}, 95 \%}, \sigma_{\mathrm{p}, 95 \%}\right)$, the $95 / 95$ coverage can be directly expressed as:

$$
\Upsilon_{95 / 95}=\mu_{p, 95 \%}+1.645 \sigma_{p, 95 \%}
$$

\section{(6) Determine licensing value of PCT}

If both parametric and nonparametric approaches and be applied to calculate the 95/95 upper tolerance limit, then the maximum value of these two calculations will be defined as the licensing value of PCT. That is:

$$
P C T_{\text {Licen sing }}=\max \left(P C T_{95 / 95}, P C T_{\text {order }}\right)
$$

where $\mathrm{PCT}_{95 / 95}$ is the PCT statistical upper bounding value determined by the parametric approach, and $\mathrm{PCT}_{\text {order }}$ is the PCT statistical upper bounding value determined by nonparametric order statistic method.

\section{Application of DRHM on PWR LBLOCA analysis with RELAP5-3D/K}

To demonstrate the benefit of DRHM method for LOCA analysis, uncertainty ranges and distributions of each essential plant parameter identified by Westinghouse (Westinghouse, 2009) are applied to analyze LBLOCA using DRHM method for the Taiwan Maanshan 3loop PWR plant. The resulting PCT by DRHM method will be compared with the PCT calculated by traditional Appendix $\mathrm{K}$ bounding parameter analysis.

In Maanshan DRHM LBLOCA analysis, 59 trails are generated by random sampling of major plant parameters listed in Table 5. The resulting PCT of each trail are shown in Figure 25 and the greatest PCT among 59 sets is $1284.6 \mathrm{~K}$. Therefore, the $\mathrm{PCT}_{95 / 95}$ estimated by order statistic method is:

$$
P C T_{95 / 95} \approx P C T_{\text {order }}=\operatorname{Max}\left[P C T_{i}, i=1,59\right]=1284.6 \mathrm{~K}
$$

Furthermore, the 59 sets of PCT were also arranged into six groups in sequential order for goodness of fitting test by using the Pearson Chi-squares test statistic (Devore, 2004):

$$
\chi^{2}=\sum_{i=1}^{k} \frac{\left(n_{i}-n \hat{p}_{i}\right)^{2}}{n \hat{p}_{i}}
$$




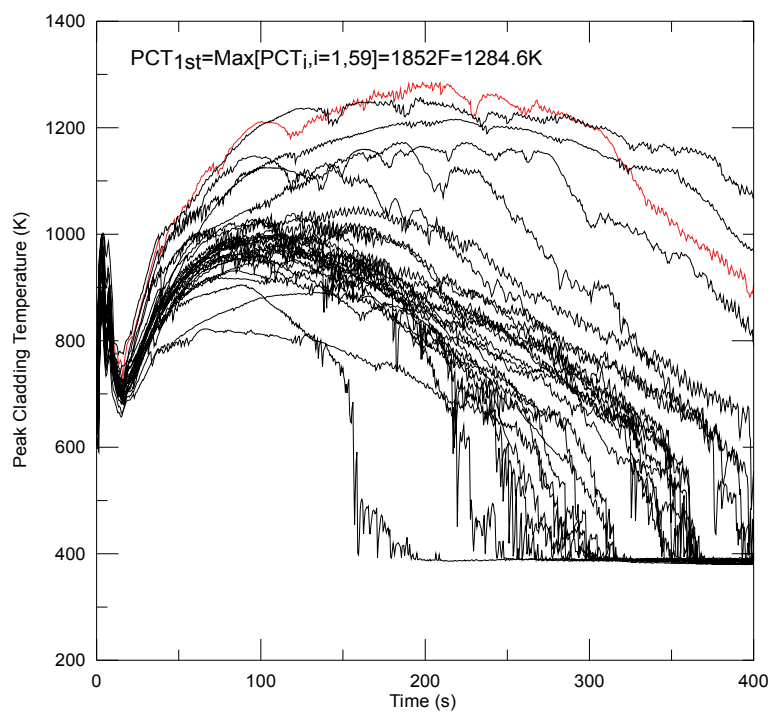

Fig. 25. Calculated PCT of each trial figure

where $\mathrm{n}$ is the total number of samples, $\mathrm{n}_{\mathrm{i}}$ is the number of samples in group $\mathrm{i}$ and $\hat{p}$ is the probability estimated by integration over group i with standard normal distribution function. The Pearson Chi-squares test statistic will be checked with the Chi-squares critical value, $\chi_{\alpha}^{2}(k-r-1)$ where $\mathrm{k}$ is the number of group $(\mathrm{k}=6)$ and $\mathrm{r}$ is the number of unknowns $(\mathrm{r}=2)$. A rejection region at $(1-\alpha)$ confidence level will be defined by $\chi_{\alpha}^{2}(k-r-1)$ as :

$$
\chi^{2} \geq \chi_{\alpha}^{2}(k-r-1)
$$

Therefore, the successful condition of goodness-of-fit test at 95\% confidence level will be:

$$
\chi^{2}<\chi_{\alpha}^{2}(k-r-1)=\chi_{0.05}^{2}(3)=7.815
$$

Since $\chi^{2}$ is 4.376 and it is less than the Chi-squares critical value $\left(\chi_{0.05}^{2}(3)=7.815\right)$, therefore the distribution normality can be accepted and the classical parametric approach can be applied to project the $\mu_{\mathrm{p}}$ and $\sigma_{\mathrm{p}}$ base on the $\mu_{\mathrm{s}}$ and $\sigma_{\mathrm{s}}$ under a giver confidence level. Under $95 \%$ confidence level the population mean value of PCT can be no greater than:

$$
\mu_{p, 95 \%} \leq\left[\mu_{s}+t_{\alpha}(n-1) * \sigma_{s} / \sqrt{n}\right]=967.6 \mathrm{~K}
$$

and the population standard deviation of PCT can be no greater than:

$$
\sigma_{p, 95 \%}^{2} \leq \frac{\sigma_{s}^{2}(n-1)}{\chi_{1-\alpha}^{2}(n-1)}=(185.6 K)^{2}
$$

As a result, the $\mathrm{PCT}_{95 / 95}$ calculated by parametric approach is:

$$
P C T_{95 / 95}=\mu_{p, 95 \%}+1.645 * \sigma_{p, 95 \%}=1272.9 \mathrm{~K}
$$


Comparing $\mathrm{PCT}_{95 / 95}(1272.9 \mathrm{~K})$ and $\mathrm{PCT}_{\text {order }}(1284.6 \mathrm{~K})$, it can be seen that statistical upper bounding values of PCT calculated by both parametric and nonparametric approaches are quite close.

To further demonstrate the benefit of DRHM method, sensitivity studies of major plant parameters were performed to identify the bounding state covering associated parameter uncertainties. In the bounding state analysis, the worse combination of either lower bounds or upper bounds of parameters are investigated. The bounding state was identified to be the upper bounding values of reactor power, $\mathrm{F}_{\mathrm{Q}}, \mathrm{F}_{\Delta \mathrm{H}}, \mathrm{T}_{\mathrm{avg}}$, and accumulator temperature and pressure, as well as the lower bounding values of system pressure, ECC temperature and accumulator water volume (Liang, 2010). Results of bounding state analysis were shown in Figure 26, and the PCT of bounding states was identified to be $1385.2 \mathrm{~K}$. Resulting PCTs from DRHM method and bounding state analysis were shown in Figure 27. It can be seen that the additional PCT margin generated by statistically combining plant status uncertainty, compared to traditional bounding state analysis, can be as great as $100 \mathrm{~K}$. A similar application of DRHM on the LOFT L2-5 based on the same plant status uncertainty was also performed (Zhang et al., 2010), and the resulting PCT analysis is shown in Figure 28. It can be observed that a comparable margin of PCT also was indicated. Furthermore, the standardized regression coefficient (SRC) method was also applied to analyze the importance of each parameter uncertainty of Maanshan plant, and the result is shown in Figure 29. It can be seen that parameter uncertainties of accumulator settings (pressure, liquid volume and temperature), ECC injection temperature, $\mathrm{T}_{\mathrm{avg}}$ and power shape are relatively important.

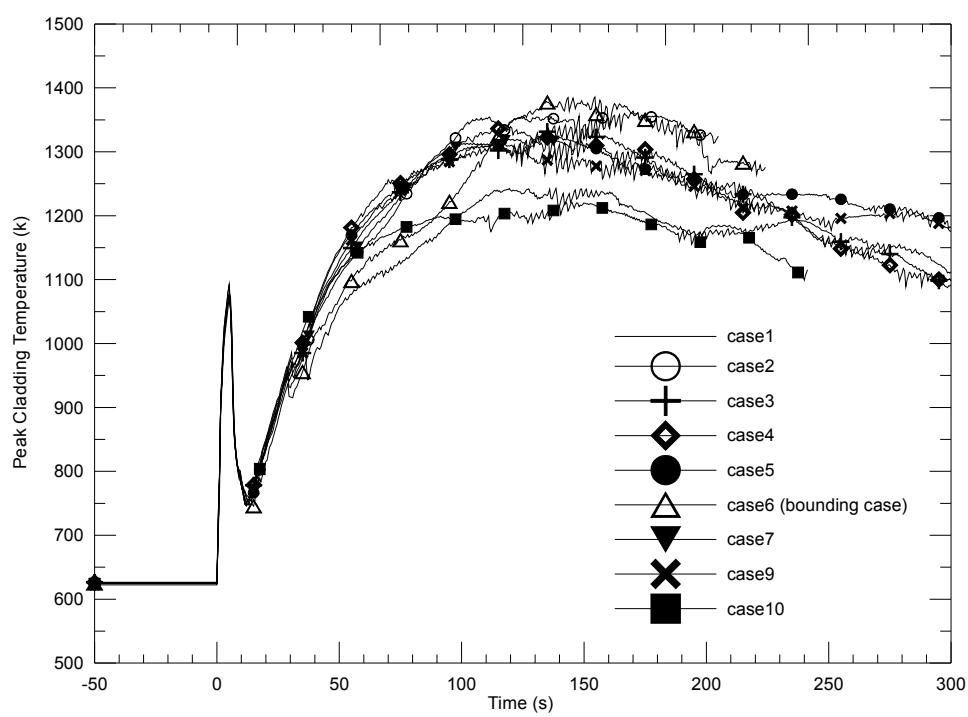

26. Bounding state analysis of PCT

\section{Conclusions}

Licensing safety analysis can only be performed by approved evaluation models, and E.M. models are composed by two major elements, which involve qualified computational codes 
and approved methodology. It is well recognized that B.E. analysis with full-scoped uncertainty quantification can provide significant safety margin than traditional conservative safety analysis, and the margin can be as great as $200 \mathrm{~K}$ for LOCA analysis. Although a bestestimate LOCA methodology can provide the greatest margin for the PCT evaluation during a LOCA, it generally takes more resources to develop. Instead, implementation of evaluation models required by Appendix $\mathrm{K}$ of $10 \mathrm{CFR} 50$ upon an advanced thermalhydraulic platform can also gain significant margin on the PCT calculation but with fewer resources. An appendix K version of RELAP5-3D has been successfully developed and through though assessments, the reasonable conservatism of RELAP5-3D/K calculation was clearly demonstrated in whole area of a LOCA event, which covering hydraulics and heat transfer in the phases of blowdown, refill and reflood.

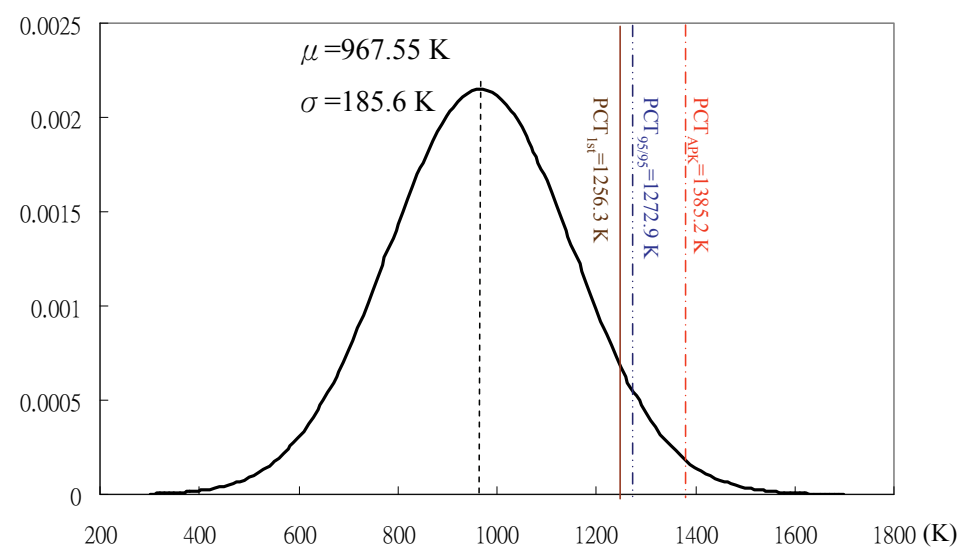

Fig. 27. Comparison of PCTs from both DRHM and bounding appendix K analysis for Maanshan PWR Plant

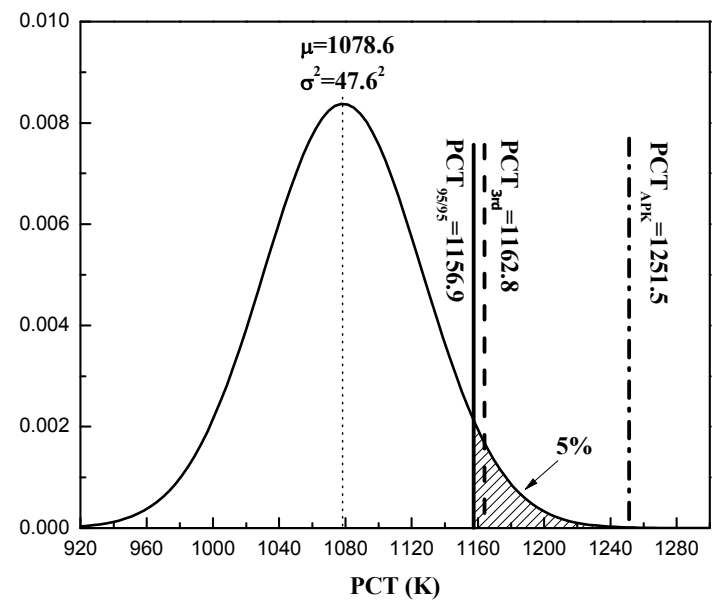

Fig. 28. Comparison of PCTs from both DRHM and bounding state analysis for LOFT L2-5 


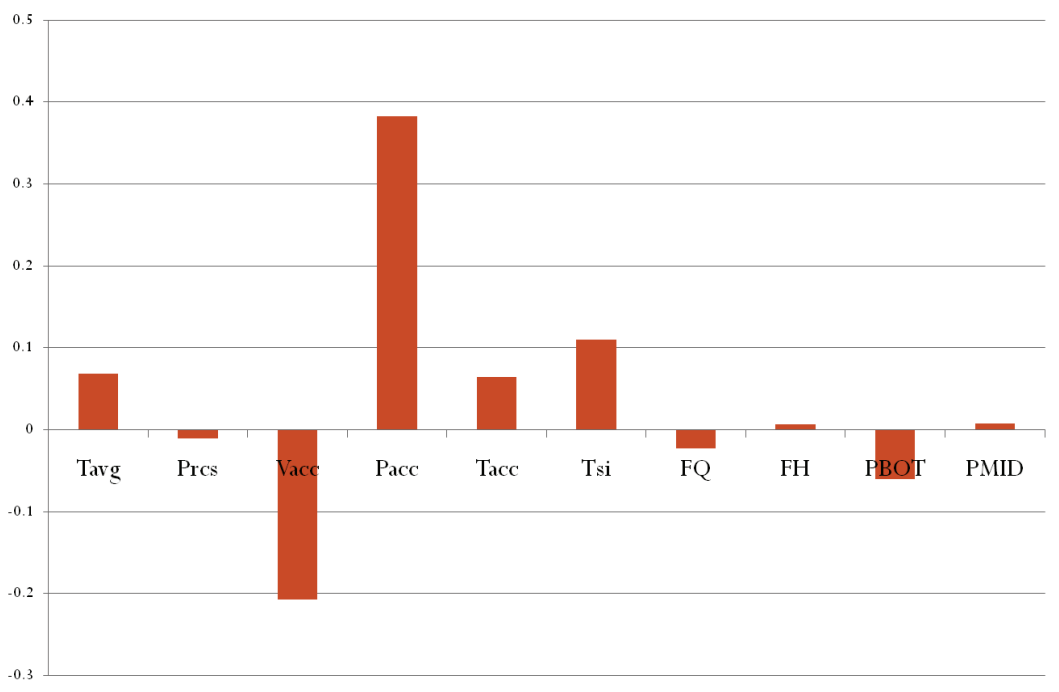

Fig. 29. Importance analysis of plant status parameters

Instead of applying a full scoped BELOCA methodology to cover both model and plant status uncertainties, a deterministic- realistic hybrid methodology (DRHM) was developed to support LOCA licensing analysis. In the DRHM methodology, Appendix K deterministic evaluation models are adopted to ensure model conservatism, while CSAU methodology is applied to quantify the effect of plant status uncertainty on PCT calculation. To ensure the model conservatism, not only physical model should satisfy requirements set forth in the Appendix K of 10 CFR 50, sensitivity studies also need to be performed to ensure a conservative plant modeling. To statistically quantify the effect of plant status uncertainty on PCT, random sampling technique is applied, and both parametric and non-parametric methods are adopted to calculate or estimate the statistical upper bounding value (95/95). When applying the DRHM for LBLOCA analysis, the margin generated can be as great as 80-100K as compared to Appendix K bounding state LOCA analysis.

\section{Reference}

Analytis, G. Th, (1996). Developmental Assessment of RELAP5/MOD3.1 with Separate Effect and Integral Test Experiments: Model Changes and Options, Nuclear Engineering and Design, 163, 125-148.

Anklam,T. M. et al., (1982). Experimental Data Report for LOFT Large Break Loss-ofCoolant Experiment L2-5, NUREG/CR2826.

Baker, Louis and Just, Louis, (1962). Studies of Metal-water Reactions at High Temperatures, ANL-6548.

Bestion, D., 1990. The Physical Closure Laws in the CATHARE Code. Nuclear Engineering and Design, 124, 229-245.

Behling, Stephen R., et al., (1981). RELAP4/MOD7-A Best Estimate Computer Program to calculate Thermal and Hydraulic Phenomena in a Nuclear Reactor or Related System, NUREG/CR-1998. 
Boyack, B., et al., (1989). Quantifying Reactor Safety Margins: Application of Code Scaling Applicability and Uncertainty (CSAU) Evaluation Methodology to a Large-Break Loss-of-Coolant Accident. NUREG/CR-5249.

Cathcart, J.V., et al., (1977). Zirconium Metal-Water Oxidation Kinetics IV. Reaction Rate Studies, ORNL/NUREG-17.

Davis, C. B., (1998). Assessment of the RELAP5 Multi-Dimensional Component ModelUsing Data from LOFT Test L2-5, INEEL-EXT-97-01325.

David, H. A. and Nagaraja, H.N., (1980). Order Statistics. A John Wiley \& Sons, INC.

Devore, Jay L., (2004). Probability and Statistics for Enginering and Sciences. The Thomsom Corporation.

Erickson, L., et al. (1977), The Marviken Full-Scale Critical Flow Tests Interim Report:Results from Test 22, MXC-222.

Grush, William H., et al., (1981). The Semiscale MOD-2C Small-Break (5\%) Configuration report for Experiment S-LH-1 and S-LH-2, EGG-LOF-5632.

Guba, A., et al., (2003). Statistical aspects of best estimate method-I. Reliability Engineering and System Safety. 80, 217-232.

Henry, et al., (1971). The Two-phase Critical Flow of One-Component Mixtures in Nozzles, Orifices, and Short Tubes, Journal of Heat Transfer, 93, 179-187.

Liang, K. S., et al., (2002). Development and Assessment of the Appendix K Version of RELAP5-3D for LOCA Licensing. Nuelear Technology, 139, 233-252.

Liang, K. S., et al., (2002). Development of LOCA Licensing Calculation Capability with RELAP5-3D in Accordance with Appendix K of 10 CFR 50. Nuclear Engineering and Design, 211, 69-84.

Liang, T. K.S., et al., (2011). Development and Application of a Deterministic-Realistic Hybrid Methodology for LOCA Licensing Analysis. Nuclear Engineering and Design, 241, 1857-1863.

Liles, D. R., et al., (1981). TRAC-PD2: advanced best-estimate computerprogram for pressurized water reactor loss-of-coolant accident analysis," NUREG/CR-2054.

Loftus, M., et al., (1980). PWR FLECHT SEASET Unblocked Bundle, Forced and Gravity Reflood Task Data Report, NUREG/CR-1531, EPRI NP-1459.

Liang, Tin-Hua, 2010. Conservative Treatment of Plant Status Measurement Uncertainty for LBLOCA Analysis. Bachelor Thesis, Shanghai Jiao-Tong University.

Moody, F. J., (1965). Maximum floe rate of a single component, two-phase mixture, Journal of Heat Transfer, Trans American Society of Mechanical Engineers, 87, No. 1.

RELAP5-3D Code Development Team, (1998). RELAP5-3D Code Manual, INEEL-EXT-9800834.

Siemens, 1988. Test No. 6 Downcomer Countercurrent Flow Test, Experimental Data Report, U9 316/88/18.

Schultz, Richard and Davis, Cliff, (1999). Recommended Models \& Correlations and Code Assessment Matrix for Creating A 10 CFR 50.46 Licensing-Version of RELAP5-3D, INEEL/EXT-98-01257.

Taiwan Power Company, (1982). Final Safety Analysis Report of Maanshan Nuclear Power Station Units 1 \& 2, Taipower Report.

Tapucu, A., et al., (1984). Experimental Study of the Diversion Cross-Flow Caused by Subchannel Blockages, EPRI NP-3459.

USNRC, (1988). Appendix K to Part 50 of 10 CFR-ECCS Evaluation model. 
USNRC, (1987). Compendium of ECCS Research for Realistic LOCA Analysis, NUREG1230.

Westinghouse Company, (2009). Best-Estimate Analysis of the Large-Break Loss-of-Coolant Accident for Maanshan Units 1 and 2 Nuclear Power Plant Using the ASTRUM Methodology. WCAP-17054-P.

Westinghouse, (1987). The 1981 Version of the Westinghouse ECCS Evaluation Model Using the BASH Code, WCAP-10266-P-A Revision 2

Yoder, G. L., et al., (1982). Dispersed Flow Film Boiling in Rod Bundle Geometry-Steady State Heat Transfer Data and Correlation Comparisons, NUREG/CR-2435, ORNL5822.

Zhang, Z. W., et al., 2010. Deterministic-Realistic Hybrid Methodology (DRHM) for LOCA Licensing Analysis - Application on LOFT L2-5 LBLOCA. Journal of Nuclear Power Engineering (China). Accepted in August 2010. 


\title{
Analysis of Error Propagation Between Software Processes
}

\author{
Sizarta Sarshar \\ Institute for Energy Technology \\ Norway
}

\section{Introduction}

All software systems can contain faults. In critical systems, this problem is alleviated by controlling the possible effects of a fault being executed, typically through techniques for achieving fault tolerance. Ensuring that failures are properly isolated, and not allowed to propagate, is essential when developing critical systems.

In much of the research on error propagation analysis the focus has been on probabilistic models. While these models are well suited for quantitative analysis, they are usually not very specific with regard to the actual mechanisms that might allow a failure to propagate between entities. Quantitative analysis is often applied on code level and not seen as influenced by and in conjunction with the operating system. A more detailed insight into the actual mechanisms can be beneficial to decide whether or not error propagation is a concern for a given source code.

A method for studying mechanisms of error propagation between software processes was proposed in (Sarshar, 2007). This chapter describes the method, which (1) facilitates the study of error propagation between software processes; (2) identifies mechanisms for error propagation; and (3) provides means to determine whether these can be automatically detected by a static analyser. In this context a process represents a program in execution, typically managed by an operating system. Processes can communicate with each other via inter-process communication and their shared resources. Examples of shared resources can be the operating system itself and the memory. The analysed problem is how one process can cause another process to fail and concerns interaction methods available in the source code of a program. The work criteria and scope are described in the following:

- $\quad$ Consider processes running on a single CPU computer with an operating system.

- $\quad$ The method should only require the source code and minimal manual input to work.

- $\quad$ The source code must compile without any errors prior to the analysis.

- $\quad$ The primary interest is to determine whether error propagation is a concern or not.

This chapter further reports on the applicability of the method in a case where a module of a core surveillance framework named SCORPIO has been analysed. The framework is a support system for nuclear power plants supporting monitoring and prediction of core conditions.

Some of the terminologies used in this chapter are briefly described in the following (Storey, 1996): 
- A fault - is a defect within the system.

- An error - is a deviation from the required operation of the system or subsystem.

- A system failure - occurs when the system fails to perform its required function.

This chapter is structured as follows: Section 2 gives a definition of error propagation, describes the mechanisms of error propagation, and previous work on the topic. Section 3 describes the proposed method for studying error propagation between software processes. Section 4 reports on the applicability of the method on one module of the SCORPIO framework. Section 5 addresses the main results. Section 6 discusses the work while section 7 provides conclusions and comments on future work.

\section{Background}

This section gives a definition of error propagation, describes the mechanisms of error propagation, operating systems and related work on the topic.

\subsection{Error propagation}

In our work, error propagation is defined as the situation where an error (or failure) propagates from one entity to another (Sarshar et al., 2007). Errors can propagate between different types of entities, including: physical entities, processes running on single or multiple CPUs, data objects in a database, functions in a program, and statements in a program. Our approach concerns propagation of errors between processes running on a single CPU computer.

Systems of interest in our work have not been limited to those that are safety critical only, e.g. systems that are directly involved in controlling a nuclear reactor. A problem of particular interest is the possible negative effect a low criticality application might have on a higher criticality application by means of error propagation because they share common resources.

Programs make use of interaction methods provided by the underlying operating system to communicate with each other, or make use of shared resources. These services are provided through the system call interface of the operating system, and are usually wrapped in functions available using standard libraries. Such interaction methods can cause errors and provide mechanisms for error propagation. A coding fault which may be manifested as an error may in principle be anything, e.g. an incorrect instruction or an erroneous data value. It may be manifested inside a local function or an external function. The propagated error need not be of the same type in different functions, e.g. an instruction error in one function realization causes a data error in another. Even if an error is propagated to one function, this does not necessarily mean that the source function fails functionally. The propagated error may only be a side-effect in this function. Another type of error related to function usage is error caused by passing illegal arguments to functions or misusing their return variables. Error propagation between two programs may occur even if both programs individually operate functionally correct. This can e.g. be caused by erroneous side effect in the implementation or execution of the programs. There are two situations possible for how one process can cause another process to fail:

- One process experiences a failure, which then causes another process to fail.

- One process propagates a fault to another process while not failing itself.

According to (Fredriksen \& Winther, 2007), possible ways of characterizing error propagation is as either intended or unintended communication or as resource conflicts. 
Error propagation in intended communication channels might consist of erroneous data transfer through parameters or global variables. Writing to the wrong addresses in memory, due e.g. to faulty pointers, exemplifies error propagation through unintended channels. Processes that demand high processor load so that other processes cannot execute are examples of resource conflicts which could cause error propagation. This indicates that error propagation between functions can occur in at least two ways:

- An error in one function is transferred via a communication channel to another function, for example through passing of arguments or return value.

- The execution of one function interacts with another function in an unintended and incorrect way, due to an error, and causes the second function to fail.

Thus error propagation can take place via the intended communication channels, i.e. those that are used by the set of functions to fulfil their tasks. It is also possible that an error in one function generates a communication channel that is not intended and propagates the error through this.

\subsection{Operating systems}

The references (Nutt, 2004; Bacon \& Harris, 2003; Bic \& Shaw, 2003; Tanenbaum \& Woodhull, 2006; Stallings, 2005) cover the basic principles of a number of important operating systems.

With respect to the Linux operating system and its kernel, one source to its understanding is given in (Bovet \& Cesati, 2003). Here, the authors describe the kernel components from how they are built to how they work. (Beck et al., 2002) explains what is in the kernel, and how to write kernel code or a kernel module. The work in (Bic \& Shaw, 2003) explains process management and interaction in the UNIX operating system, and in (Pinkert \& Wear, 1989), the authors describe all major components of an operating system down to the pseudo code level. The authors employ a generic approach and present the fundamental concepts involved, alternative policies from which a designer can choose, and illustrative mechanisms for implementing selected policies.

In (Kropp et al., 1998), the Ballista methodology is applied on several implementations of the POSIX operating system C language API. The methodology is for automatic creation and execution of invalid input robustness tests designed to detect crashes and hangs caused by invalid inputs to function calls. The Ballista POSIX robustness test suite was ported to ten operating systems where even in the best case, about half of the functions had at least one robustness failure. The results illustrate that error propagation is a concern in operating systems.

A study of operating system errors found by automatic and static compiler analysis applied to the Linux and OpenBSD kernels is reported in (Chou et al., 2001). Static analysis is applied uniformly to the entire kernel source. The scope of errors in the study is limited to those found by their automatic tools. These bugs are mostly straightforward source-level errors. They do not directly track problems with performance, high-level design, user space programs, or other facets of a complete system. (Engler et al., 2000) examines features of operating system errors found automatically by compiler extensions. Some of the results they present include the distribution of errors in the kernel: the vast majority of bugs are in drivers.

Our approach focuses on analysing user space programs. We examine how the operating system manages processes and provides services to user programs through the system call interface, but we do not analyse its code. We assume that the operating system performs its 
intended functions correctly and that it is implemented correctly. Instead, we analyse the system call interface and other process interaction mechanisms to identify whether these may cause error propagation.

\subsection{Related work}

Error propagation analysis has to a large extent been focused on probabilistic approaches (Hiller et al., 2001, Jhumka et al., 2001; Nassar et al., 2004; Abdelmoez et al., 2004) and model based approaches (Voas, 1997; Michael \& Jones, 1997; Goradia, 1993).

In (Hiller et al., 2001), the concept of error permeability is introduced as a basic measure upon which a set of related measures is defined. These measures guide the process of analysing the vulnerability of software to find the modules that are most likely to propagate errors. Based on the analysis performed with error permeability and its related measures, how to select suitable locations for error detection mechanisms (EDMs) and error recovery mechanisms (ERMs) are described. Furthermore, a method for experimental estimation of error permeability, based in fault injection, is described and the software of a real embedded control system analysed to show the type of results obtainable by the analysis framework. The results show that the developed framework is very useful for analysing error propagation and software vulnerability, and for deciding where to place EDMs and ERMs. The paper (Jhumka et al., 2001), assess the impact of inter-modular error propagation between embedded software systems. They develop an analytical framework which enables to systematically design software modules so the inter-modular error propagation is reduced by design. The framework is developed using influence and separation metrics, then the framework is validated using fault injection experiments, which artificially inject faults and errors into the system. Influence metric is in their paper referred to as the probability of a module directly influencing another module, i.e., when no other module is considered while separation metric is referred to as the probability of a module not influencing another one when all other modules are considered. The results showed that the analytical framework can predict the influence value between a pair of modules very accurately.

The study of software architectures is an important discipline in software engineering, due to its emphasis on large scale composition of software products, and its support for emerging software engineering paradigms such as product line engineering, component based software engineering, and software evolution. Architectural attributes differ from code-level software attributes in that they focus on the level of components and connectors, and that they are meaningful for architecture. In (Abdelmoez et al., 2004), focus is on a specific architectural attribute, which is the error propagation probability throughout the architecture, e.g. the probability that an error arising in one component propagates to other components. Formulas for estimating these probabilities using architectural level information are introduced, analysed, and validated.

In (Voas, 1997), error propagation between commercial-off-the-shelf (COTS) components is analysed using an approach termed interface propagation analysis (IPA). IPA is a faultinjection based technique for injecting 'garbage' into the interfaces between components and then observing how that garbage propagates through the system. An example, if component A produces information that is input to component $B$, then the information is corrupted using fault injection techniques. This simulates the failure of component A. After this corrupt information is passed into B, IPA analyses the behaviour of B (or components 
executed after B) to the information. IPA analyses the behaviour of a component by looking for specific outputs that the user wants to be on the lookout for.

(Michael \& Jones, 1997) presents an empirical study of an important aspect of software defect behaviour: the propagation of data-state errors. A data-state error occurs when a fault is executed and affects a program's data-state, and it is said to propagate if it affects the outcome of the execution. The results show that data-state errors appear to have a property that is quite useful when simulating faulty code: for a given input, it appears that either all data state errors injected at a given location tends to propagate to the output, or else none of them do. These results are interesting, because of what they indicate about the behaviour of data-state errors in software. They suggest that data state errors behave in an orderly way, and that the behaviour of software may not be as unpredictable as it could theoretically be. Additionally, if all faults behave the same for a given input and a given location, then one can use simulation to get a good picture of how faults behave, regardless of whether the simulated faults are representative of real faults.

Goradia (Goradia, 1993) addresses test effectiveness, i.e. the ability of a test to detect faults. This thesis suggests an analytical approach, introducing a technique of dynamic impact analysis using impact graphs to estimate the error propagation behaviour of various potential sources of errors in the execution. The empirical results in the thesis provide evidence indicating a strong correlation between impact strength and error propagation. The time complexity of dynamic impact analysis is shown to be linear with respect to the original execution time and experimental measurements indicate that the constant proportionality is a small number ranging from 2.5 to 14.5. Together these results indicate that they have been fairly successful in their goal of designing a cost effective technique to estimate error propagation. However, they also indicate that to reach the full potential benefits of the technique the accuracy of the estimate needs to be improved significantly. In particular, better heuristics are needed for handling reference impact and program components tolerant to errors in control paths.

Research on error propagation has identified frameworks and techniques for estimating error propagation, e.g. in (Jhumka et al., 2001; Goradia, 1993). In difference, our goal is to identify sources and mechanisms for error propagation in order to identify potential error propagation scenarios and remove the failures to improve software.

\section{Method of analysis}

A method for analysing the interfaces between processes and their shared resources in the search for mechanisms for error propagation is provided in (Sarshar, 2007; Sarshar et al., 2007). This section describes this method which starts out by investigating how processes are managed in the relevant operating system, enabling us to identify process characteristics relevant to error propagation. The output of this step includes a list of system calls in the system call interface of the operating system. Secondly, the identified interaction methods are analysed using Failure Mode and Effect Analysis (FMEA) (Stamatis, 1995). This approach helps to identify types of code characteristics that might be a concern in relation to error propagation. The method of analysis can be summarized in three steps:

1. Examination of the operating system for how it interacts with and manages processes to obtain an overview of e.g. a list of system calls and common resources;

2. Analysis of the interaction methods using Failure Mode and Effect Analysis (FMEA) to identify possible faults that can cause error propagation to occur; and 
3. Determination of how the mechanisms can be recognized in source code.

The method was developed for $C$ code under the Linux operating system as a case. $C$ was chosen because it is a widely used programming language and Linux because it is an open source operating system. In section 4 , the method is applied on one module of the SCORPIO framework.

\subsection{How processes run in operating systems}

Processes are managed by the operating system. An operating system provides a variety of services that programs can utilise using special instructions called system calls. The typical functions of an operating systems kernel are: process management, memory management, input and output management, and support functions. In Linux, the kernel components managing processes are the following:

- Signals: the kernel uses signals to call into a process.

- System calls (explained below).

- Process manager and scheduler: creates, manages and schedules processes.

- Virtual memory: allocates and manages virtual memory for processes.

A process interface to the operating system is either a result of the use of system calls or through direct memory access. Use of a pointer in the $C$ language is an example of accessing memory without the use of the system call interface. In Linux, system calls are implemented in the kernel. When a program makes a system call, the arguments are handled in the kernel, which takes over the execution of the program until the call completes (Mitchell et al., 2001). System calls are usually wrapped in the standard C library and may require some parameters and return a value. Examples of system calls are low-level input and output functions, such as open() and $\operatorname{read}()$. The system calls of Linux can be grouped into the following categories (Silberschatz et al., 2005; Bic \& Shaw, 2003):

- Process management: create/terminate process, load, execute, end, abort, get/set process attributes, wait for time, wait/signal event, allocate and free memory.

- File management: create/delete file, open, close, read, write, reposition, get/set file attributes.

- Device management: request/release device, read, write, reposition, get/set attributes, logically attach or detach device.

- Inter-process communication: the transfer of data among processes.

- Communications: create, delete connection, end, receive messages, transfer status information, attach or detach remote device.

- Miscellaneous services: get/set time or date, system data.

The essence of our approach is to identify mechanisms for error propagation that have characteristics detectable when analysing source code. We can therefore narrow down our scope to include those parts of the operating system which fulfil this requirement. The kernel components that allow interaction directly in source code of a program include the system call interface and signals. Language specific traps and pitfalls (Hatton, 1995; Koenig, 1989) might also open ways for an error to propagate. Programming errors can give variables incorrect values that can lead to failures. Our analysis does not specifically address general programming errors, but errors related to invoking system calls.

We focus here on programs written to run in user space, and exclude programs written for kernel space, as they have their own kernel API which provide services for kernel programming. 
Figure 1 shows a simple illustration of the channels available in source code of a program for interaction with the operating system and its resources. These include the system call interface, signals, and traps and faults, with arrows indicating the interactions.

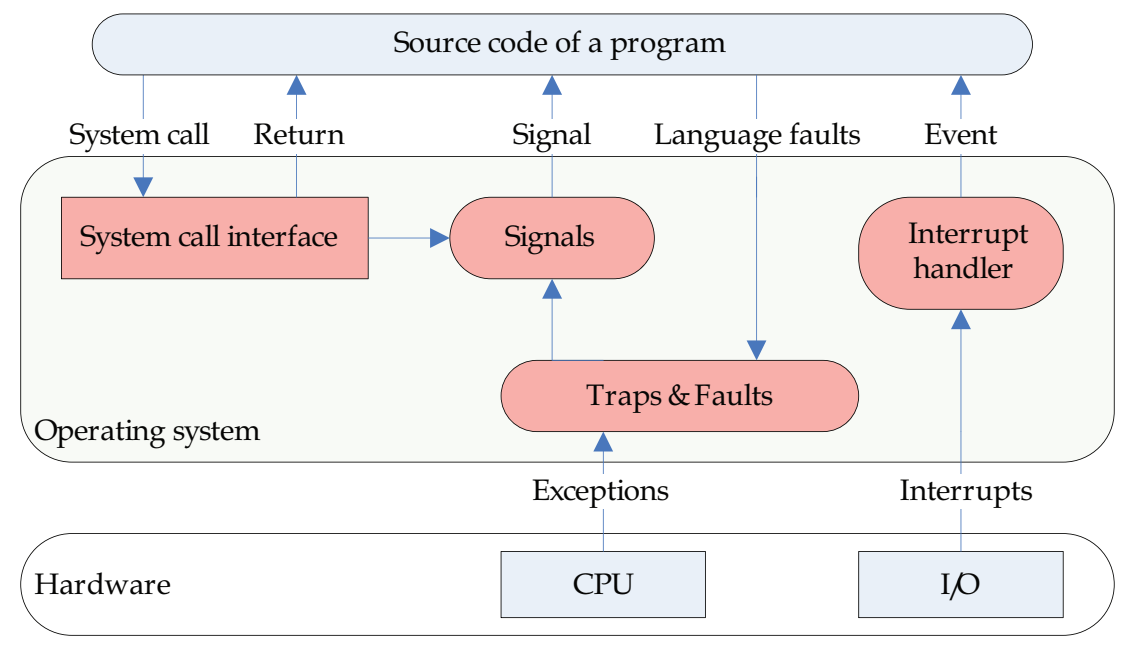

Fig. 1. Illustration of the interaction methods of the operating system on processes

An interrupt is a condition that can cause the normal execution of instructions to be altered. Interrupts and exceptions are known as signals and are used to notify a process of certain faults by the kernel (Pinkert \& Wear, 1989):

- Completion of an input or output operation.

- Division by zero.

- Arithmetic overflow or underflow.

- Arrival of a message from another system.

- Passage of an amount of time.

- Power failure.

- Memory parity error.

- Memory protect violation.

A signal might also be altered from another program using the system call interface.

In source code, interaction with the operating system is only available through the system call interface. It is therefore not necessary to examine how processes are handled and managed at deeper levels.

\subsection{Identify system call failures causing error propagation}

In the proposed method, each system call is analysed using FMEA. The purpose is to identify failure modes that can cause errors to propagate to other processes or the operating system. The focus in this analysis is on failure modes that have characteristics in the source code of a program.

FMEA is a well-known analysis method for risk and reliability analysis. The basis for this analysis is a description of a system in terms of its components and the communication between them. For each of the components in the system, the aim is to identify all potential 
modes of failure, by investigating the following questions for each component and communication unit, based on the FMEA framework:

- What can go wrong? (failure mode)

- How can this occur? (failure cause/mechanism)

- Which consequences will this have on the further actions and messages? (failure effects via error propagation)

In our method, the FMEA is targeted on the system call as a component and the focus is on its usage in source code of a program. Once the failure modes have been identified, we determine their potential effects on local and system processes to determine whether any of these can cause error propagation. This can be done in two ways:

- The effect is described in the system call documentation as an error the function can return.

- $\quad$ The effect is determined using fault injection in test programs.

The failure effects will provide information on the severity of failures and help us provide possible mitigation actions.

\subsection{Identify the failure mode characteristics in source code}

The aim of step three of the method is to determine whether the failure modes identified in the previous step are present in the source code of a program. For each failure mode that can cause error propagation, we determine its characteristics in code so it can be detected when analysing an application's source code. We then examine some existing code analysis tools to check whether any of these will recognise the failure modes, and if they do, determine whether they identify all of them. The next step is to develop an algorithm for identifying the failure modes in source code, including how to traverse and check the code for the identified failures. The result is a prototype tool which demonstrates that failures causing error propagation can be detected by analysing source code.

The steps of the method are performed only once for an operating system and programming language combination. The prototype tool is run for each application source code we wish to analyse for error propagation.

\section{Case on SCORPIO}

SCORPIO (Surveillance of reactor CORe by Picture On-line display) is a core surveillance framework for nuclear power plants, and is developed at the Institute for Energy Technology (IFE). The framework is a support system for the monitoring and prediction of pressurized water reactors (PWR), boiling water reactors (BWR) and VVER (Russian design series of PWRs) core conditions and is running on several reactors worldwide (Barmsnes et al., 1997). The framework has passed established system tests including factory acceptance testing and site acceptance testing.

The general SCORPIO framework is illustrated in Figure 2. The module administrator is a program that connects the modules to the graphical user interface made using ProcSee (IFE, 2010). ProcSee is a versatile software tool for developing and displaying dynamic graphical user interfaces, particularly aimed at process monitoring and control. All data exchanged between the modules and the operator is transmitted through this program. The Software Bus handles the communication between all modules. In the case study, the input data processing (IDATP) module of the framework has been assessed. The IDATP module consists of 30 files and approximately 5300 lines of code. 


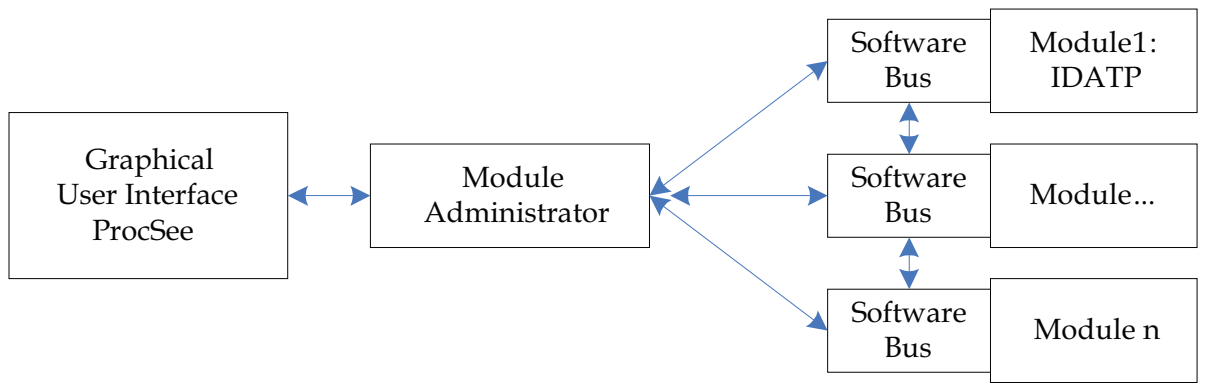

Fig. 2. The general SCORPIO framework

The source code of the IDATP module is first examined to identify which calls it performs to system and library functions. The attributes passed to these external functions and the values retrieved are stored for later analysis.

\begin{tabular}{|l|l|l|l|}
\hline Function & System call & Library call & Description \\
\hline close & $\mathrm{x}$ & $\mathrm{x}$ & Close a file descriptor \\
\hline execvp & & $\mathrm{x}$ & Execute file \\
\hline fclose & & $\mathrm{x}$ & Close a stream file \\
\hline fopen & $\mathrm{x}$ & Open a stream file; convert file to stream \\
\hline fprintf & & $\mathrm{x}$ & $\begin{array}{l}\text { Formatted output conversion to a given } \\
\text { stream }\end{array}$ \\
\hline fscanf & & $\mathrm{x}$ & Input format conversion \\
\hline memcpy & & $\mathrm{x}$ & $\begin{array}{l}\text { Copies } n \text { bytes from memory area source } \\
\text { to memory area destination }\end{array}$ \\
\hline memset & & & Fill memory with a constant byte \\
\hline pipe & & $\mathrm{x}$ & Creates a pair of file descriptors \\
\hline printf & & $\mathrm{x}$ & $\begin{array}{l}\text { Formatted output } \\
\text { standard out stream }\end{array}$ \\
\hline shmget & $\mathrm{x}$ & $\mathrm{x}$ & Allocate a new shared memory segment \\
\hline signal & & $\mathrm{x}$ & Signal handling \\
\hline sprintf & & $\mathrm{x}$ & $\begin{array}{l}\text { Formatted output conversion to a given } \\
\text { character string }\end{array}$ \\
\hline sscanf & & Input format conversion \\
\hline strcat & & Concatenate two strings \\
\hline strcmp & & Compare two strings \\
\hline strlcpy & & Copy string \\
\hline strlen & & Calculate length of string \\
\hline strncmp & & Compare two strings \\
\hline
\end{tabular}

Table 1. Analysed system and library calls 


\subsection{Applying the analysis}

Each system and library function of the IDATP module is analysed using FMEA with focus on identifying failure modes that can cause the module or the system itself to encounter failure. A failure mode specifies how an entity may fail. An entity may be e.g. a variable, used as either an argument passed to a function or used as a return variable.

The system manuals for these calls form the basis for this analysis. The IDATP module makes use of several system and library calls. A subset of 19 of these functions, listed in Table 1, were analysed using FMEA.

To exemplify the analysis, we focus on the shmget() system call to demonstrate the usage of the method in the following. Thus emphasis is on the steps involved in performing the analysis and understanding the analysis object.

The shmget() system call creates or allocates a new shared memory segment for interprocess communication (IPC) between processes. This IPC provides a channel for communication between processes using the memory. The main services related to shared memory are shmget(), shmat(), shmctl(), and shmdt(). Other calls related to shared memory include services for managing semaphores. The relation between these calls are as follows: A process starts by issuing a shmget() system call to create a new shared memory with the required size. After obtaining the IPC resource identifier, the process invokes the shmat() system call, which returns the starting address of the new region within the process address space. When the process wishes to detach the shared memory from its address space, it invokes the shmdt() system call.

We begin with an examination of the system call documentation and then perform FMEA on the function. When performing the analysis, the aim is to identify failure modes caused by wrong usage of the service in source code, and determine their effects on local and system processes. The focus is on those failure modes causing error propagation.

The synopsis for the shmget() function:

\# include <sys/types.h>

int shmget(key_t key, size_t size, int shmflg);

The shmget() function returns the identifier of the shared memory segment associated with the value of the argument key. A new shared memory segment, with size equal to the value of size rounded up to a multiple of PAGE_SIZE, is created if:

- $\quad$ key has the value IPC_PRIVATE, or

- $\quad$ key is not IPC_PRIVATE, no shared memory segment corresponding to key exists, and IPC_CREAT is specified in shmflg

PAGE_SIZE, IPC_PRIVATE and IPC_CREAT are definitions within the operating system. IPC_PRIVATE is not a flag field but a key_t type. If this special value is used for key the system call ignores everything but the least significant 9 bits of shmflg and creates a new shared memory segment, on success. The value of shmflg's least significant 9 bits specify the permission mode, the permissions granted to the owner, group, and world.

The FMEA process starts with identifying failure modes. Table 2 illustrates identified failure modes for the shmflg parameter of shmget(). This is an excerpt from the complete FMEA sheet for this function.

For each identified failure mode, we now examine its effects on the process itself (indicates "local effect" in the FMEA sheet) and on other processes (indicates "system effect" in the FMEA form). Some of these failure modes are detected by the system call; the function exits 
with return value equal to -1 , which indicates an error, and the external variable errno is set appropriately. Many of these are described in the manual pages and can be identified as the failure effect on the local process. However, not all failure modes are represented as error cases in the manual pages. We make use of test programs to identify these.

\begin{tabular}{|l|l|l|}
\hline Reference & Variable & Failure mode \\
\hline F.29.3.A & Parameter shmflg & Not specified at all \\
\hline F.29.3.B & Parameter shmflg & $\begin{array}{l}\text { Is not one of IPC_CREAT, IPC_EXCL, SHM_HUGETLB } \\
\text { or SHM_NORESERVE }\end{array}$ \\
\hline F.29.3.C & Parameter shmflg & Is of wrong type \\
\hline F.29.3.D & Parameter shmflg & No permission mode is set \\
\hline F.29.3.E & Parameter shmflg & $\begin{array}{l}\text { Access permission is given to all users, instead of user } \\
\text { only }\end{array}$ \\
\hline F.29.3.F & Parameter shmflg & Permission mode is write when it should have been read \\
\hline F.29.3.G & Parameter shmflg & Permission mode is read when it should have been write \\
\hline F.29.3.H & Parameter shmflg & Permission mode is set without user access \\
\hline F.29.3.I & Parameter shmflg & IPC_EXCL specified without IPC_CREAT \\
\hline F.29.3.J & Parameter shmflg & $\begin{array}{l}\text { Wrong flag specified i.e. IPC_CREAT I IPC_EXCL when } \\
\text { not intended }\end{array}$ \\
\hline
\end{tabular}

Table 2. Failure modes for parameter shmflg for the shmget() system call

A test program is written to execute a failure mode while the failure effect is monitored. Such test programs have the possibility to execute an injected failure mode.

Based on such test programs one can determine the effect of failure modes. E.g. the effect for failure mode F.29.3.D "no permission mode is set" was determined to be: no processes can access the shared memory segment unless they are privileged. Checking the value of the parameter shmflg to identify whether the permission mode is set is easily done performing static analysis, thus this failure mode can be detected in source code.

Table 3 shows the complete FMEA for the failure modes related to the shmflg parameter of shmget() from Table 2 .

Similarly, the remaining system and library calls are analysed. The failure modes identified in the analysis of these calls are related to passing of arguments and handling return values, and can be grouped as follows:

- Argument refers to uninitialized variable/pointer.

- Argument is of different type than specified in function definition.

- Argument refers to null-pointer.

- Argument is freed.

- Argument refers outside an arrays size.

- Argument is an array of chars which is not null-terminated when required.

- Return value is not retrieved from a non-void function.

- Return value is not checked to determine successful call.

- Return value is not used in scope.

These failure modes are then compared with the checks that existing tools perform to determine whether any of these are present in their checks. 


\begin{tabular}{|c|c|c|c|c|}
\hline Ref. & Failure mode & Local effect & System effect & Conclusion \\
\hline F.29.3.A & Not specified at all & - & - & Does not compile \\
\hline F.29.3.B & $\begin{array}{l}\text { Is not one of } \\
\text { IPC_CREAT, } \\
\text { IPC_EXCL, } \\
\text { SHM_HUGETLB or } \\
\text { SHM_NORESERVE }\end{array}$ & $\begin{array}{l}\text { Unknown flag and } \\
\text { permission is set }\end{array}$ & $\begin{array}{l}\text { Segment may not } \\
\text { be created or } \\
\text { accessed }\end{array}$ & $\begin{array}{l}\text { Detectability in } \\
\text { source code must } \\
\text { be determined }\end{array}$ \\
\hline F.29.3.C & Is of wrong type & $\begin{array}{l}\text { Uses the int value } \\
\text { of the type if } \\
\text { possible, unknown } \\
\text { flag and } \\
\text { permissions are } \\
\text { set on segment }\end{array}$ & $\begin{array}{l}\text { Segment may not } \\
\text { be created or } \\
\text { accessed }\end{array}$ & $\begin{array}{l}\text { Detectable in } \\
\text { source code }\end{array}$ \\
\hline F.29.3.D & $\begin{array}{l}\text { No permission mode is } \\
\text { set }\end{array}$ & $\begin{array}{l}\text { The process } \\
\text { cannot access the } \\
\text { shared memory } \\
\text { segment unless it } \\
\text { is run in } \\
\text { privileged mode }\end{array}$ & $\begin{array}{l}\text { Other processes } \\
\text { cannot access the } \\
\text { shared memory } \\
\text { segment unless } \\
\text { they are run in } \\
\text { privileged mode }\end{array}$ & $\begin{array}{l}\text { Detectable in } \\
\text { source code }\end{array}$ \\
\hline F.29.3.E & $\begin{array}{l}\text { Access permission is } \\
\text { given to all users, } \\
\text { instead of user only }\end{array}$ & - & $\begin{array}{l}\text { Other users can } \\
\text { access the shared } \\
\text { segment }\end{array}$ & $\begin{array}{l}\text { Detectability in } \\
\text { source code must } \\
\text { be determined }\end{array}$ \\
\hline F.29.3.F & $\begin{array}{l}\text { Permission mode is } \\
\text { write when it should } \\
\text { have been read }\end{array}$ & $\begin{array}{l}\text { Can write to } \\
\text { segment when not } \\
\text { intended }\end{array}$ & $\begin{array}{l}\text { Other processes } \\
\text { can write to } \\
\text { segment when } \\
\text { not intended }\end{array}$ & $\begin{array}{l}\text { Detectability in } \\
\text { source code must } \\
\text { be determined }\end{array}$ \\
\hline F.29.3.G & $\begin{array}{l}\text { Permission mode is } \\
\text { read when it should } \\
\text { have been write }\end{array}$ & $\begin{array}{l}\text { Cannot write to } \\
\text { segment }\end{array}$ & $\begin{array}{l}\text { Other processes } \\
\text { cannot write to } \\
\text { segment }\end{array}$ & $\begin{array}{l}\text { Detectability in } \\
\text { source code must } \\
\text { be determined }\end{array}$ \\
\hline F.29.3.H & $\begin{array}{l}\text { Permission mode is set } \\
\text { without user access }\end{array}$ & $\begin{array}{l}\text { The process } \\
\text { cannot access the } \\
\text { shared segment } \\
\text { unless it is run in } \\
\text { privileged mode }\end{array}$ & - & $\begin{array}{l}\text { Detectable in } \\
\text { source code }\end{array}$ \\
\hline F.29.3.I & $\begin{array}{l}\text { IPC_EXCL specified } \\
\text { without IPC_CREAT }\end{array}$ & $\begin{array}{l}\text { Exits with error if } \\
\text { segment already } \\
\text { exists }\end{array}$ & - & $\begin{array}{l}\text { Detectable in } \\
\text { source code }\end{array}$ \\
\hline F.29.3.J & $\begin{array}{l}\text { Wrong flag specified } \\
\text { i.e. IPC_CREAT | } \\
\text { IPC_EXCL when not } \\
\text { intended }\end{array}$ & $\begin{array}{l}\text { Tries to create } \\
\text { instead of getting } \\
\text { identifier for the } \\
\text { shared segment }\end{array}$ & - & $\begin{array}{l}\text { Detectability in } \\
\text { source code must } \\
\text { be determined }\end{array}$ \\
\hline
\end{tabular}

Table 3. Example of FMEA for the parameter shmflg of the shmget() system call 


\subsection{Analysis tools}

There are several existing analysis tools which identify different types of errors. These tools include both static and dynamic analysis methods. In (Sarshar, 2007), over 20 tools were examined and compared to determine what kind of errors they detect. Of these tools, one group performs checks on passing of arguments, another group warns if a return value is not retrieved and a third group warns about sequential issues. The tool Splint (Secure Programming Lint, 2008) was the only tool which gave warnings on all three groups. Therefore, Splint was chosen for assessment of our source code in part three. None of the tools performed checks on argument values and they did not check all argument types to be correct.

Based on the available documentation on existing analysis tools, we assume that some tools can check arguments and some tools can check the return value for the following issues:

- Types - assignment of variables, passing arguments of different type than function expects.

- Null pointers - a common cause of failures is when a null pointer is dereferenced.

- Definitions - all function parameters and global variables used by a function must be defined before a call, and the return value must be defined after the call.

- Allocations - concerns: reallocating storage when there are other live references to the same storage, or failing to reallocate storage before the last reference to it is lost.

- Aliasing - program errors often result when there us unexpected aliasing between parameters, return value, and global variables.

An important difference between the identified failure modes from the FMEA and the checks existing tools perform is to check a variable value in the context of the relevant function it is passed to. E.g. the system call shmget() has an argument of type size_t; As a data type, the variable must be checked to be of correct type and its value must be checked to be within the variable limits. Most existing analysis tools do these checks. But, in the context of the function the argument is passed to, the variable must be checked to determine e.g. whether its value is smaller than the maximum size of a shared memory segment (set by the operating system).

The next step was to assess the source code for the identified failure modes that existing tools do not check for. To automate this process, we made use of a prototype tool described in (Sarshar \& Winther, 2008). The tool was modified for this study and its purpose was to identify different attributes for each argument that was passed to a given function. If statically detectable, the following attributes were determined; the argument type, value, name, whether it was an array and if so, its size. This information was used as input to check the arguments for the potential failure modes. Several of these checks were automated; however, a majority was done manually by examination of the argument attributes against the FMEA sheets for each function.

Splint was also applied on the source code of our case study with the checks described in the list above. However, the tool can also do more powerful checks enabled by source code annotations. Annotations are stylized comments that documents assumptions about functions, variables, parameters and types and follow a predefined syntax. To use the more powerful checks, the source code must be edited to add notations. This requires time and effort and was not applied in this case study. The use of annotations for more powerful checks applies to most static analysis tools. 


\section{Results}

A subset of 19 external calls has been analysed using FMEA to identify potential failure modes that can cause a process to fail or propagate error. The examined functions were called 309 places in the source code.

In 242 of the cases, the return value from an external call was not retrieved or checked. In general, the return value often indicates whether a function succeeded or failed for some reason. If such failure is not handled, unexpected runtime errors can occur in a software system. As an example, consider an application which writes some data to a file regularly. The file is opened for reading successfully and the write function is called without checking its return value. If the file was inaccessible (e.g. lost connection to server) the write function would return a value indicating an error. If the error is not handled explicitly, a runtime error may occur. Such an error often causes the operating system to give an error message to the user and then terminates the application that caused the error. All unsaved data will be lost in such events. However, not all calls are this crucial; it is more vital that the return value from an open or write function is handled than the return value of a print to screen function. 76 of the ignored return cases were for a print function.

Several of the examined functions had potential failure modes regarding the content of arguments they receive. In example, char arrays passed to a group of functions must be nullterminated and for another group they must not contain a given character. Our assessment of the code did not identify any of these failure modes in the module.

The source code was also assessed using the tool Splint which gave near 2000 warnings on the source code of the module. Table 4 (Sarshar, 2009) presents warnings given by Splint and number of instances. In general, the tool reports many false positive warnings (which add noise to the results and make it harder to spot the real problems). Though the number of cases for the warnings on incompatible types and dangerous comparison are equal, there is no relation between them.

\begin{tabular}{|l|l|}
\hline Warning on & Cases \\
\hline Incompatible types & 444 \\
\hline Dangerous comparison & 444 \\
\hline Variable declared but not used & 50 \\
\hline Value used before definition & 160 \\
\hline Variable initialized to null value & 14 \\
\hline Dangerous assignments & 237 \\
\hline Test expression issues & 163 \\
\hline Storage not released before return & 37 \\
\hline Return value ignored & 212 \\
\hline Possible buffer overflow with sprintf() & 23 \\
\hline Arrow access of non-pointer & 54 \\
\hline Other warnings & 162 \\
\hline
\end{tabular}

Table 4. Group of warnings given by the tool Splint 
Assessment of many existing systems in the industry can only be performed on the available source code, and often, the specification is not available. This is where static analysis is useful, some tools only need the source code to perform their analysis. However, if annotations are necessary to perform an assessment, expertise on the system is required.

The method proposed to use FMEA on system calls to identify potential failure modes and then assess the source code for these potential failures. The intention was not to develop yet another tool, therefore the identified failure modes were checked against the ones that existing tools check. An interesting approach would be, if possible, to write these failure modes as additional checks for existing tools. A disadvantage of the FMEA analysis is that it only identifies a small fraction of the potential failure modes and it requires expert knowledge on the system calls.

System and library calls are complex functions which interact with the kernel of the operating system. The process of analysing such functions takes time and effort, but it only needs to be performed once for each function. The result from this analysis indicates that it is necessary to examine the source code of applications for failures related to system call usage.

The source code of the input data processing module of the SCORPIO framework was assessed using our approach and using the tool Splint. The user of analysis tools must be critical to the results as all vulnerabilities are not guaranteed to be found, and identified vulnerabilities are not all real problems. Splint gave a lot of warnings which were false positives while the checks from the FMEA performed by us gave few false positives. The reason for this is that we used a prototype tool to help us identify variable attributes, but the checks were done manually. Performing manual checks is time consuming, but reduces the chance of false positives since the analyser is required to have insight of the application. Furthermore, it is difficult, if not impossible, to control and check the value of variables that are passed to system services when performing static analysis.

Through the process of analysing the source code of the module, failure modes with the potential to cause harm at runtime as an effect of fault triggering and error propagation have been identified. These failure modes are related to usage of services provided by the underlying operating system. Though the arguments sent to such functions are valid and in accordance with the documentation, the majority of the potential failure modes detected in the code were related to handling of return values from these functions.

We did not expect that this assessment would identify any serious failures in the code, and the result demonstrates that this expectation is valid. Potential failures related to usage of operating system services would have been identified using our method and none of the potential failures identified is likely to cause the module to fail. However, taking these results into account in new releases of the module will reduce its vulnerability.

\section{Discussion}

The methodology was applied on a subset of system calls, some of them related to shared memory. This target was found to be suitable because it involved an intended channel for communication between processes through a shared resource; the memory. We also performed FMEA on other system calls to evaluate whether the method is applicable to a wider class of functions and not restricted to those related to shared memory. The errors identified in this approach are erroneous values in the variables passed to the system call interface and errors caused when return, or modified, pointer variables are not handled 
properly. From the analysis we know not only which functions behave non-robustly, but also the specific input that results in errors and exceptions being thrown by the operating system. This simplifies identification of the characteristics an error has in code, making it easier to locate errors.

The method for analysing error propagation between processes primarily focuses on how the process of interest can interact with and affect the environment (the operating system and other processes). A complementary approach could be to analyse how a process can be affected by its (execution) environment. In (Johansson et al., 2007), the authors inject faults in the interface between drivers and the operating system, and then monitor the effect of these faults in the application layer. This is an example where processes in the application layer are affected by their execution environment. Comparing this method to our approach, it is clear that both methods make use of fault injection to determine different types of failure effects on user programs. However, the examination in (Johansson et al., 2007) only concerns incorrect values passed from the driver interface to the operating system. Passing of incorrect values from one component to another is a mechanism for error propagation and relates to problems for intended communication channels. Fault injection is just one method to evaluate process robustness in regards to incorrect values in arguments. In our work, we examine the failure effects of several mechanisms: passing of arguments and return values, usage of return values, system-wide limitations, and sequential issues. These methods complement each other.

Understanding the failure and error propagation mechanisms in software-based systems will provide the knowledge to develop defences and avoid such mechanisms in software. It is therefore important to be aware of the limitations for the proposed approach. This analysis only identifies failure modes related to the usage of system calls in source code. Other mechanisms for error propagation that do not involve usage of the system call interface will not be covered by this approach. This approach, however, complements existing methods and static analysis tools. An infinite loop structure in code is one example of a failure mode that does not make use of system calls. This failure mode can cause error propagation because it uses a lot of CPU time/resources.

The FMEA method worked well on system calls and identified failure modes that could cause error propagation between processes. However, the identified failure modes from the FMEA do not apply directly to other operating systems. A new analysis must be performed for a new programming language and operating system combination. Even though several operating systems provide the same functionality, e.g. usage of shared memory, the implementation of the service will be different. Thus, some of the failure modes may be similar, yet their effects may not. And, in contrast to general FMEA approaches which analyse functionality of software systems, our aim was to identify failure modes related to the interaction of a program with operating system services.

\section{Conclusion}

The analysis and results from this case shows that the approach facilitates the detection of potential failure modes related to the use of the system calls in operating systems. However, this is without further analysis about their actual impact in the SCORPIO framework. Future extension of the work can include examining the potential impact of these failure modes. With so many potential failure modes it also seems that there needs to be some way to prioritize or target the "important" failures that should be fixed based on the study. For 
example, the missing return values seem to become critical errors only under maintenance, if the return values can change. Even though this is valuable to uncover, it would be more valuable to quantify which potential failures would be critical if they occurred under the current operational mode and which would not. This would help to indicate the usefulness of the technique and provide some evidence that the failures occur with sufficient frequency to justify the definition of a technique that targets them. Further extension of the work can include exploring alternative techniques or quantify effort required to conduct this type of analysis to make it easier to determine the trade-offs of using this technique in practice, providing a quantitative analysis of the types of failure modes the analysis uncover and providing usage guidelines to the practitioner.

\section{References}

Abdelmoez, W.; Nassar, D.; Shereshevsky, M.; Gradetsky, N.; Gunnalan, R.; Ammar, H. H.; Yu, B. \& Mili, A. (2004). Error Propagation in Software Architectures, metrics, Proceedings of International Symposium on Software Metrics No10, pp. 384-393, Chicago IL, ETATS-UNIS, USA, September 11, 2004.

Bacon, J. \& Harris, T. (2003). Operating Systems - Concurrent and distributed Software Design, 1st ed., Great Britain: Pearson Education Limited, 2003.

Barmsnes, K. A.; Johnsen, T. \& Sundling, C-V. (1997). Implementation of Graphical User Interfaces in Nuclear Applications, Proceedings of Topical Meeting on IEC of VVER, Prague, April 21-24, 1997.

Beck, H.; Bohme, H.; Dziadzka, M.; Kunitz, U.; Magnus, R.; Schroter, C. \& Verworner, D. (2002). Linux Kernel Programming, 3rd ed., Great Britain: Pearson Education Limited, 2002.

Bic, L. F. \& Shaw, A. C. (2003). Operating Systems Principles, USA: Pearson Education, Inc., 2003

Bovet, D.P. \& Cesati, M. (2003). Understanding the Linux Kernel, 2nd ed., USA: O'Reilly \& Associates, Inc., 2003.

Chou, A.; Yang, J.; Chelf, B.; Hallem, S. \& Engler, D. R. (2001). An Empirical Study of Operating Systems Errors, Proceedings of the 18th Symposium on Operating System Principles (SOSP), Chateau Lake Louise, Banff, Canada, October, 2001.

Engler, D.R.; Chelf, B.; Chou, A. \& Hallem, S. (2000). Checking System Rules Using SystemSpecific, Programmer-Written compiler Extensions, Proceedings of Operating systems Design and Implementation (OSDI), San Diego, California, USA, October, 2000.

Fredriksen, R. \& Winther, R. (2007). Challenges Related to Error Propagation in Software Systems, Proceedings of Risk, Reliability and Societal Safety (ESREL), pp. 83-90, ISBN 978-0-415-44783-6, Stavanger, Norway, June 25-27, 2007.

Goradia, T. (1993). Dynamic Impact Analysis: A Cost-Effective Technique to Enforce Error Propagation, Proceedings of the International Symposium on software Testing and Analysis, pp. 171-181, 1993.

Hatton, L. (1995). Safer C: Developing for High-Integrati and Safety-Critical Systems, Great Britain: Mcraw-hill, 1995.

Hiller, M.; Jhumka, A. \& Suri, N. (2001). An Approach to Analysing the Propagation of Data Errors in Software. Dependable Systems and Networks (DSN), 2001.

IFE (Institute for Energy Technology) (2010). ProcSee, available from: http://www.ife.no/departments/visual_interface_technologies/products/procsee

Jhumka, A.; Hiller, M. \& Suri, N. (2001). Proceedings of 20th IEEE Symposium on Reliable and Distributed Systems, pp. 152-161, New Orleans, LA, USA, October 23-31, 2001. 
Johansson, A.; Suri, N. \& Murphy, B. (2007). On the Impact of Injection Triggers for OS Robustness Evaluation, Proceedings of the 18th International Symposium on software Reliability Engineering (ISSRE), pp. 127-136, 2007.

Koenig, A. (1989). C Traps and Pitfalls, USA: Addison-Wesley, 1989.

Kropp, N. P.; Koopman, P. J. Jr. \& Siewiorek, D. P. (1998). Automated Robustness Testing of Off-the-Shelf Software Components, Proceedings of the Symposium on Fault-Tolerant Computing, pp. 230-239, 1998.

Michael, C. \& Jones, R. (1997). On the Uniformity of Error Propagation in Software, Proceedings of the $12^{\text {th }}$ Annual Conference on Computer Assurance (COMPASS), pp. 6876, 1997.

Mitchell, M.; Oldman, J. \& Samuel, A. (2001). Advanced Linux Programming, $1^{\text {st }}$ ed., USA: New Riders Publishing, pp. 45-55, 2001.

Nassar, D.; Rabie, W.; Shereshevsky, M.; Gradetsky, N. \& Ammar, H. (2004). Estimating Error Propagation Probabilities in Software Architectures, Proceedings of International Symposium on Software Metrics No10, pp. 384-393, Chicago IL, ETATSUNIS, USA, September 11, 2004.

Nutt, G. (2004). Operating Systems, 3rd ed., USA: Pearson Education, Inc., 2004.

Pinkert, J. R. \& Wear, L. L. (1989). Operating Systems - Concepts, Policies, and Mechanisms, USA: Prentice-Hall, Inc., 1989.

Sarshar, S.; Simensen, J.E.; Winther, R. \& Fredriksen, R. (2007). Analysis of Error Propagation Mechanisms between Software Processes, Proceedings of Risk, Reliability and Societal Safety (ESREL), pp. 91-98, Taylor \& Francis, ISBN 978-0-415-44783-6, Stavanger, Norway, June 25-27, 2007.

Sarshar, S. (2007). Analysis of Error Propagation between Software Processes in Source Code, Master thesis at Østfold University College, Norway, 2007.

Sarshar, S. \& Winther, R. (2008). Automatic Source Code Analysis of Failure Modes Causing Error Propagation, Proceedings of Risk, Reliability and Societal Safety (ESREL), pp. 183190, Taylor \& Francis, ISBN 978-0-415-48514-2, Valencia, Spain, September 22-24, 2008.

Sarshar, S. (2009). Performing Code Interface Analysis on the SCORPIO Core Surveillance Framework", Proceedings of the 6th American Nuclear Society International Topical Meeting on Nuclear Plant Instrumentation, Control, and Human-Machine Interface Technologies (NPICEHMIT), American Nuclear Society, LaGrange Park, IL, Knoxville, Tennessee, April 5-9, 2009.

Secure Programming Lint (2008). Annotation-Assisted Lightweight Static Checking, Available from: http://www.splint.org, 2008.

Silberschatz, A.; Galvin, P. B. \& Gagne, G. (2005). Operating System Concepts, $7^{\text {th }}$ ed., USA: John Wiley \& Sons, Inc., pp. 43-55, 2005.

Stallings, W. (2005). Operating Systems - Internals and Design Principles, $5^{\text {th }}$ ed., USA: Pearson Education, Inc., 2005.

Stamatis, D. (1995). Failure Mode and Effect Analysis: FMEA from Theory to Execution, American Society for Quality, USA, 1995.

Storey, N. (1996). Safety-Critical Computer Systems, Britain: Pearson Education Limited, 1996

Tanenbaum, A. S. \& Woodhull, A. S. (2006). Operating Systems - Design and Implementation, $3^{\text {rd }}$ ed., USA: Pearson Education, Inc., 2006

Voas, J. (1997). Error Propagation analysis in COTS Systems, IEEE Computing and Control Engineering Journal, 8(6):269-272, December, 1997. 


\title{
Thermal-Hydraulic Analysis in Support of Plant Operation
}

\author{
Francesc Reventós \\ Technical University of Catalonia \\ Spain
}

\section{Introduction}

Many different engineering tasks are performed in support of operation of nuclear power plants with the aim of carrying out an effective and safe exploitation. Among such activities maintenance, core follow-up, refuelling and analyzing operating experience are the most commonly cited. Thermal-hydraulic analysis is an important issue that could help many different aspects of the engineering activity taking care of plant operation.

Integral Plant Models prepared using system codes are a valuable tool to carry out analytical activities devoted to contribute to engineering support to plant operation.

Most of the issues and tasks presented in the chapter are part of the job description of the so called thermalhydraulic analyst supporting plant operation (Reventós, 2008). Usually, this analyst is an engineer belonging to the technical team that takes care of engineering plant support. In many plants such engineer takes care of plant models and he personally performs at least the first approach analysis of any of the issues involved. Depending on the amount of work needed to carry out each specific analysis the whole work or only a part of it is done by him. In the first case the benefits are clear since he knows the plant and he uses the information produced or treated by the team he belongs to. In the second case, when the amount of work is too large, the thermalhydraulic analyst will take care of the technical subcontracting of the analysis. The benefits in this latter case are also clear since he is coordinating a task well known to his own calculating experience.

This chapter has three different sections. The first one gives some detail on thermalhydraulic analysis tasks related to operation. The second clarifies some features that are specific of Integral Plant Model. Especially, it establishes how the nodalization is qualified. Finally, the third briefly presents some relevant results of one example of analysis performed in such context along with the concise description of other two cases.

\section{Thermalhydraulic analysis tasks related to Nuclear Power Plant (NPP) operation}

A tentative list of issues concerning the contents of this section could be the following: Thermal-hydraulic analysis of Probabilistic Safety Assessment (PSA) and Emergency Operating Procedures (EOP) sequences, Dialogue with regulatory body and fuel designer, Analysis of actual transients, NPP start-up tests analysis, Transient analysis for training support, Design modifications and Improvement of plant availability. 
Safety Reports from International Atomic Energy Agency (IAEA) and specially (IAEA, 2002) and (IAEA, 2006) are strongly related to the mentioned list of tasks. These documents were developed based on broad international consensus and they describe types and rules for performing computational analyses devoted to both being built and operating plants. The purpose of this section is not to describe every related task but to add some aspects that are specific of the functions of the analyst working in support of plant operation.

In fact every utility or every manager having the responsibility of organizing engineering support to plant operation decides which tasks are to be fulfilled by the thermalhydraulic analyst. Since it is clear that the best estimate (BE) prediction of a scenario helps communication on any engineering subject related to dynamic behaviour, it is difficult to know what comes first task definition or analytical capabilities. In many occasions managers decide to integrate the analyst in the engineering group dealing with support to plant operation. Group objectives are clear and depending on the proved analytical capabilities of the simulating tools the thermalhydraulic analyst results become useful for different purposes.

The thermal-hydraulic analysis of PSA sequences is a well known engineering activity. PSA sequences analyses are normally performed using integral BE plant models (Reventós, 2007a) (Reventós, 2006). These are a kind of studies that fit perfectly in the job description of the analyst. Again IAEA rules are normally followed and no additional comments are needed. It is also one of these pieces of work that are usually subcontracted to engineering companies due to the amount calculations needed.

Something similar occurs with the analyses devoted to Emergency Operating Procedures (EOPs) validation. In fact they are, from calculation point of view quite close to those related to PSA. Integral Plant Models prepared using BE system codes are again the suitable tool for the analysis.

As an enhancement the last two activities BE calculation results are also useful to the dialogue with regulatory body or fuel designer. Sensitivity calculations on the treated scenarios help understanding the related engineering judgements.

The analysis of operating experience is a quite complex activity that needs to coordinate the efforts of many different engineering teams belonging to the utility itself and external organizations. The study of actual transients occurred in the plant usually involves different approaches. The simulation of actual transients produces in-depth knowledge of their dynamic behaviour. It is also helpful to investigate and to determine the cause-effect relationships of the occurred transient (Reventós, 1993) (Reventós, 2001) (Llopis, 1993a). One of the most powerful arguments in favour of these kinds of analysis is that they provide the possibility of generating time trends of functions and magnitudes that are not collected by plant instrumentation. Last section of this chapter shows an example of this capability.

As it usually happens with experiments performed in test facilities, start-up tests of NPP need also pre and post test calculations. The pre-test or the predictive study of NPP start-up tests is extremely helpful for the test coordinator in order to avoid unexpected interactions and delays that could give rise to economic losses (Llopis, 1993b). Competitiveness goals of the electricity business have led the company running the plant to minimize the number of start-up tests to be performed. This kind of analysis helps to reduce the number of tests to only those that have proven benefits for both operation and safety. The expected benefit is usually either better knowledge of dynamic behaviour or the correct performance of a system or instrument. Apart from these important activities related to start-up tests, 
standard post-test analyses could also become very significant. Important adjustments of the plant model arise very often from the studies carried out as post-test analyses.

Besides these basic activities presented above there are other less common tasks that are carried out in many cases by the analyst. Among such tasks one can find activities related to training and also all the studies intending to clarify systems interactions.

The former include not only the validation of plant training simulators but also the analyses devoted to direct training actions.

The latter needs some explanation on how it came to engineering organizations. The progress of BE codes development came out with new codes with a high performance in simulating not only core or primary system thermalhydraulics but also controls, other hydrodynamic systems and core neutron-kinetics. This development ended with powerful control blocks, with a huge amount of logic and real variables and the capability of using control equations to simulate many different phenomena quite far from the original intended uses. Such innovative uses started with plant design modifications analyses, having the goal of establishing the impact that modifications in components or systems may have on the interactive global operation of the plant. And later on, other subjects came out like set-point adjustment, other technological changes, or even the improvement of plant availability.

\section{Integral plant model}

This section clarifies some features that are specific of Integral Plant Models. Especially, it establishes how the nodalization is qualified. For this reason this part will emphasize two different aspects. The first is related to the main differences between the nodalizations used in safety analysis and those used for this particular aim. The second one is the special qualification process needed for the model in order to properly fulfil the objective of contributing to plant operation support. Although extensive technical literature exists aimed at establishing the requirements needed to qualify a NPP model, most of this literature is focused on qualifying a model for licensing uses.

The first task to be performed is selecting the code to be used. Taking into account the list of issues presented in the last section, a BE code seems the right option. Maybe some aspects of the presented issues could be solved by conservative or simplified codes but it is generally accepted that a BE code provides the right approach for support of operation issues.

The options are not many, since only a few BE codes exist. Codes like Relap5, TRACE or Cathare are the most used currently. The right code has to be available for the engineering organization and properly documented and maintained. Documentation of a code is the so called code manual which includes a huge amount of information on how the code calculates as well as how one can certify that the calculation is performed properly. Code manuals follow an established organization that includes:

- Code structure

- $\quad$ System models and solution methods

- User's guide and input requirements

- Developmental assessment

- Models and correlations

- User's guidelines

- Validation of numerical techniques

- Summaries of independent code assessment reports

- Programmers manual 
Once the code has been selected, the next task is analysing the available design information of the plant such as drawings, equipment specifications, data sheets and descriptive documents produced by component manufacturers. All this information is used to prepare the nodalization: control volumes are defined with junction data, as well as all the details needed. Figure 1 shows an example on the relationship between a drawing of a detail of the reactor vessel and the corresponding nodalization diagram. Following the code manual all this information is organized as an input deck that will be read by the code. The input deck use to be a text file. All these tasks are properly documented in a "Nodalization Description Report". Strict maintenance is performed by up-dating both the input deck and the descriptive documents. Quality assurance of these activities is an important issue related to establishing procedures and keeping data bases for control changes.

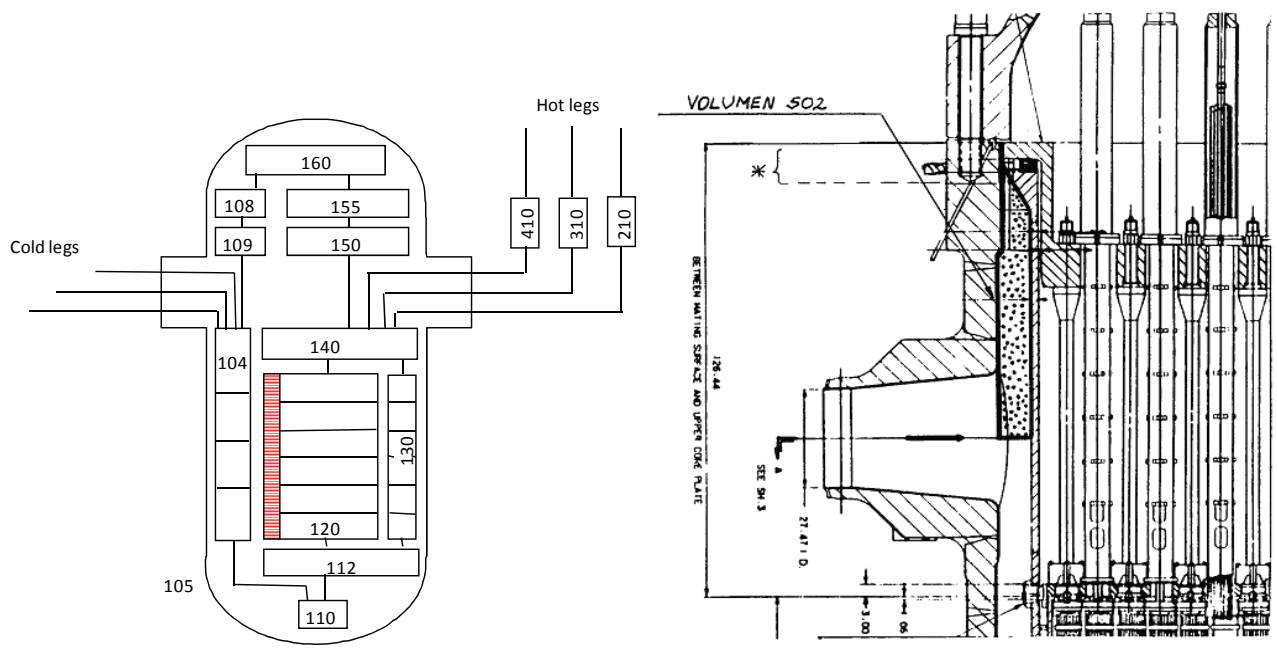

Fig. 1. Reactor vessel: detailed drawing and nodalization

The main differences between the nodalizations used in safety analysis and those used for this particular aim are drawn from what is specified in the previous section. Since the analyst wants his plant model being valid for a large amount of cases, the model has to be quite complete.

Examples of such differences are the following. For instance, in nodalizations used in safety analysis, control systems are often omitted, since many analyses have to be performed assuming that they fail.

Manual action model can be another example. In many safety scenarios manual actions are not considered following the design basis specification. A similar comment can be done for neutron-kinetic model, interlocks or non-safety systems.

If the analyst wants to be able to study actual transients in normal or abnormal operation, at a definite time of the cycle, the most relevant control systems have to be implemented in the model as well as neutron-kinetic model, interlocks and non-safety systems. The limits or borders of the nodalization depend on the scenario to be simulated and following the purpose of the analyst tasks the model intents to cover an important part of the plant. For this reason such nodalizations are often called Integral Plant Models. Sometimes, once a 
particular study is started, some specific development is performed in order to complete the scope needed to simulate the scenario to be analyzed.

Figure 2 shows an example of the main nodalization diagram of an Integral Plant Model used for such purpose. Some other diagrams representing: safety injection systems, steam lines, main and auxiliary feed-water, and detailed diagrams of vessel, pressurizer and steam generators are also part of the supporting documentation.

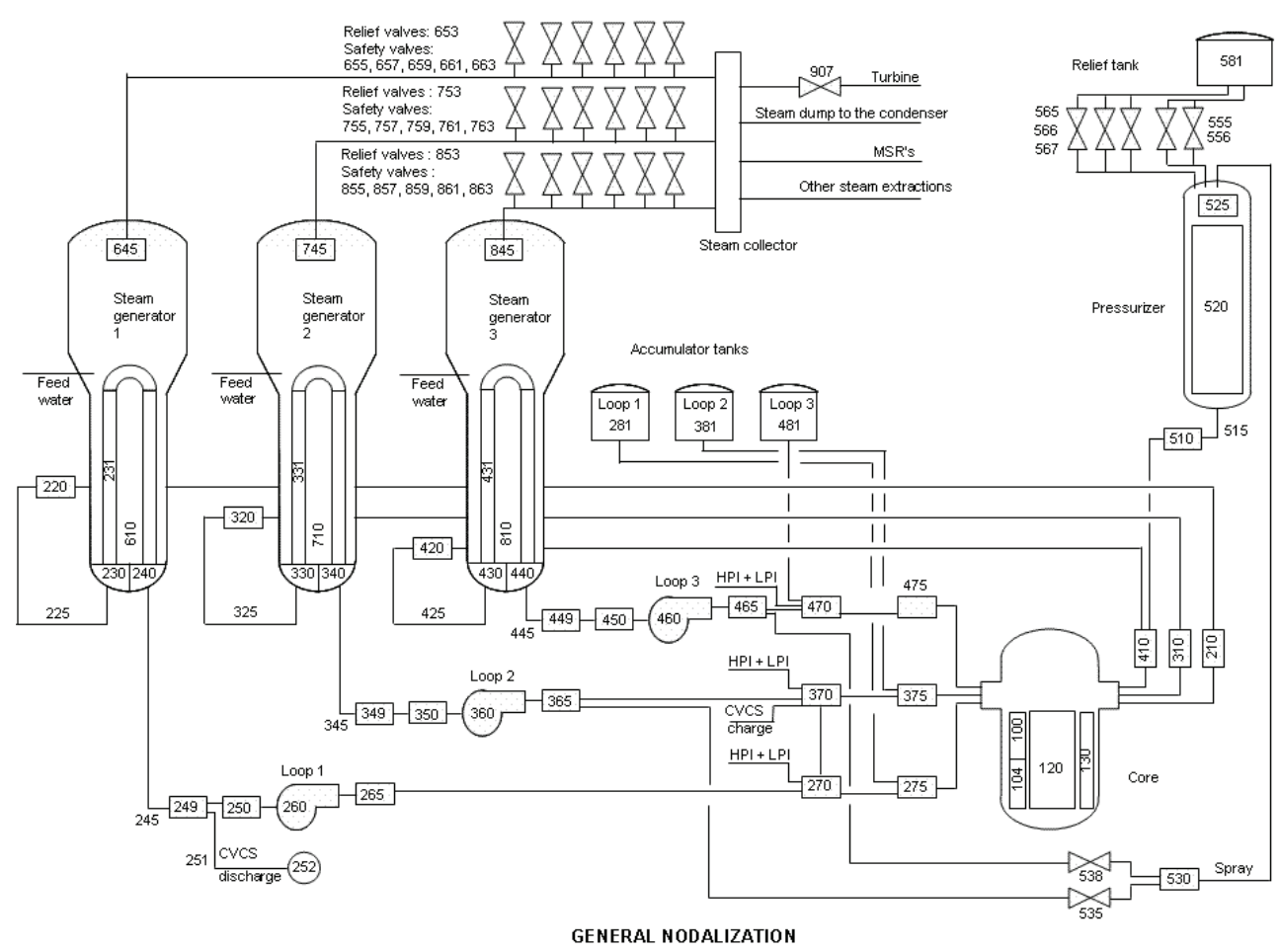

Fig. 2. Example of main nodalization diagram

Figure 3 shows an example of a logic diagram implemented for another similar model. Quite a great number of control systems with a degree of complexity as in the case of Figure 3 are usually included in an Integral Plant Model.

Once the model has been prepared including all specific features and the input deck describing the plant is ready, the nodalization has to be qualified. A general strategy (Reventos, 2007a) distinguishes between Basic and Advanced Qualification Processes. Both are considering comparisons between the results of the simulations and data collected by plant instrumentation. As a general statement when predictions are reasonably close to actual time trends (see figure 4) the nodalization is considered to be validated. The number of compared parameters and their significance are an important point. Figure 4 shows the time trends of Steam Generator narrow range level in a load rejection transient. As can be seen, the predicted values are in close agreement with actual ones. 

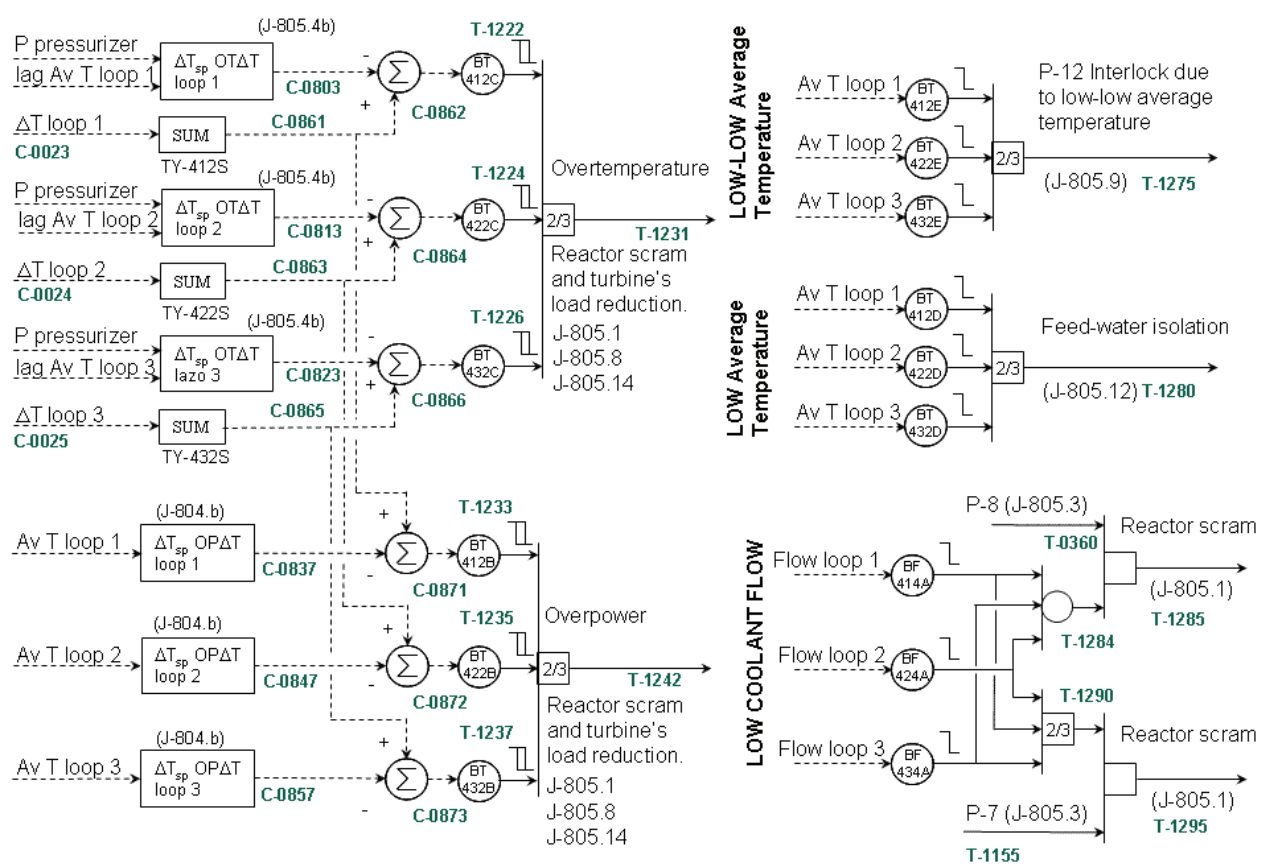

REACTOR SCRAM SIGNALS PRODUCED BY PRIMARY COOLANT SYSTEM

Fig. 3. Example of a logic diagram

Basic Qualification process is usually performed following guidelines and widely recommended good practices. Recommendations can be found in different publications among them: the code manual itself (see chapter called User's guidelines) and also some specific reports of international organizations like OECD/CSNI (Ashey, 1998) and IAEA (IAEA, 2002). Different existing methodologies devoted to certify the acceptability of the qualification process are available. Most of them follow similar rules and steps like steady state and transient qualification (Berthon, 2005) and Kv scaled calculations (Martínez, 2008). The methodology described in the article (Petruzzi, 2005) is especially relevant and complete since it uses not only qualitative comparisons of calculated and experimental time trends but also it evaluates quantitatively the accuracy of the simulation.

The second level is the so-called AQP and is carried out if the model is to be used not only for licensing but also for each type of transient analysis presented in the previous section. The description of the methodology can be found in (Reventós, 2007a), along with some detail on the most specific difficulties usually encountered in such process.

The most important thing that needs to be considered in this additional plant specific process is the availability of plant data related to meaningful sets of qualification transients. Dynamic transient behaviour is not frequent in operating plants. The number of scheduled tests is also limited. Some start-up tests are performed at the beginning of each cycle, but others are only repeated from time to time.

Plants can undergo major design modifications such as Steam Generator replacement. As validation is plant specific, changes have to be considered. It could be said that after any 
important modification a new model qualification is needed. Fortunately, some essential systems and components remain the same and this will be useful in order to reduce engineering effort.

\section{Vandellòs II N.P.P. - Load rejection from $100 \%$ to $50 \%$.}

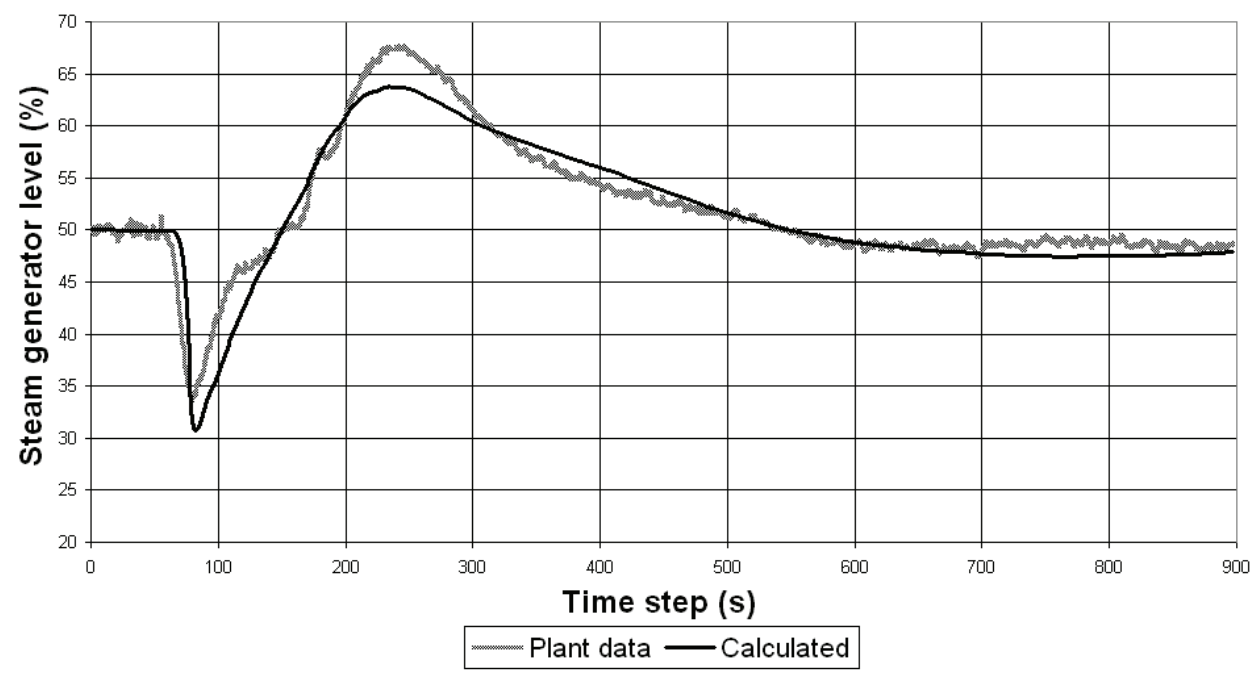

Fig. 4. Comparison between calculated and predicted time trends

The concept of configuration is highly helpful for a fruitful use of the limited amount of available plant data of meaningful sets of transients suitable for validation. A plant configuration is the state of the plant for a period of time in which no important changes are made. It is understood that important changes are those related to heavy equipment replacement, essential system modification and power level variation. Examples of configuration descriptions of a given plant along with some comments on distinctive features could help to illustrate this issue.

Some conclusions can be drawn from the analysis of the information above. Old configurations have usually two important problems: low quality of data and a lack of detailed as-built information. In spite of this, they have a very interesting advantage: a large amount of unexpected transients. Current configurations also have advantages and disadvantages. Among the former: high quality of data and proper documentation of new systems. Among the latter: the limited number of unexpected transients and start-up tests.

Taking all the above-mentioned features into account, a careful selection of transients is usually performed along with the identification of systems that remain unchanged in the different configurations. These identification tasks define the qualification process, which relies on the comparison of plant data and model prediction for the selected transients.

The AQP is a progressive activity that confers an important level of qualification to the Integral Plant Models and provides the procedure to improve this level gradually after the analysis of transients that may occur in the future.

The plan has three main steps: 
- $\quad$ Preparing the qualification matrix.

- Executing assessment calculations.

- Performing comparison and final evaluation.

\begin{tabular}{|c|l|l|}
\hline $\begin{array}{c}\text { Configuration } \\
\text { Period }\end{array}$ & Distinctive features & Remarks \\
\hline $\begin{array}{c}1^{\text {st }} \\
(1983-1995)\end{array}$ & Old Steam Generators & $\begin{array}{l}\text { Difficulties in data recording } \\
\text { Large number of unexpected } \\
\text { transients }\end{array}$ \\
\hline $\begin{array}{c}2^{\text {nd }} \\
\text { configuration }\end{array}$ & $\begin{array}{l}\text { New Feed Water Control System } \\
\text { (1995-1999) }\end{array}$ & $\begin{array}{l}\text { New value of average temperature } \\
\text { Complete set of start-up tests }\end{array}$ \\
\hline $\begin{array}{c}3^{\text {rd }} \\
\text { configuration } \\
(1999-2002)\end{array}$ & Approximately $8 \%$ power up-rating & $\begin{array}{l}\text { Very few unexpected transients } \\
\text { Selected start-up tests }\end{array}$ \\
\hline $\begin{array}{c}4^{\text {th }} \\
\text { configuration } \\
(2002-\text { today })\end{array}$ & Approximately 1\% power up-rating & $\begin{array}{l}\text { Very few unexpected transients } \\
\text { Limited number of start-up tests }\end{array}$ \\
\hline
\end{tabular}

Table 1. Ascó configurations

The qualification matrix is prepared by establishing a list of plant transients suitable for qualification and another list of systems and components needed for advanced qualification. The first list is prepared in a comprehensive way. Some decisions can be made in order to obtain a good level of qualification with reasonable effort. Some configuration can be interesting due to the number of unexpected transients but the engineering effort to maintain the corresponding model could be excessive. In this case, it is better to discard the configuration provided that there are sufficient recorded transients in the rest of the configurations.

The second list has to be prepared to identify which systems and components have had a significant effect on the transients analysed to date or intended to be analysed in a near future. Following what has been established in the previous section, this includes a complete set of calculations related to PSA and others to EOP analysis.

Once both lists were ready, the information was organized, as can be seen in Figures 5 and 6. The former is the qualification matrix and the latter shows a detailed part of it. Each transient is set to a column (i) and each system or component to a line (j). In the box corresponding to column $i$ and line $j$, one or two names of parameters are set. These are the key parameters to check the correct functioning of system $j$, which is properly recorded in transient $\mathrm{i}$.

If the key parameter of a system or component is properly documented in more than one transient, the input is adjusted to simultaneously match, or at least to reasonably approach, all the different recorded behaviours. In this way the matrix helps the analyst to keep track of his experience and make it useful for future modelling tasks. 


\begin{tabular}{|c|c|c|c|c|c|c|c|c|}
\hline & & $\begin{array}{c}\text { *transient } 1 \text { * } \\
\text { Reactor trip due to } \\
\text { low flow }\end{array}$ & $\begin{array}{c}* \text { transient } 2 * \\
\text { Reactor trip due to } \\
\text { low SG level }\end{array}$ & 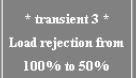 & 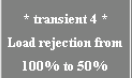 & $\begin{array}{l}{ }^{*} \text { transient } 5 \text { * } \\
\text { Load swing firm } \\
75 \% \text { to } 65 \%\end{array}$ & $\begin{array}{l}\text { "transient 6* } \\
\text { Load swing from } \\
65 \% \text { to } 75 \%\end{array}$ & $\begin{array}{c}\text { * transient } 7 \text { * } \\
\text { Load rejection from } \\
100 \% \text { to } 50 \%\end{array}$ \\
\hline $\begin{array}{l}\text { Pressurizer's safety } \\
\text { system }\end{array}$ & $\begin{array}{l}\text { valves } \\
\text { control }\end{array}$ & - & - & - & - & - & - & - \\
\hline $\begin{array}{l}\text { Pressurizer's relief } \\
\text { system }\end{array}$ & $\begin{array}{l}\text { valves } \\
\text { control }\end{array}$ & - & - & - & - & - & - & - \\
\hline Pressurizer spray & $\begin{array}{l}\text { valves } \\
\text { control }\end{array}$ & $\begin{array}{c}\text { Spray valve's } \\
\text { position/Primary } \\
\text { pression }\end{array}$ & $\begin{array}{c}\text { Spray valve's } \\
\text { position/Primary } \\
\text { pression }\end{array}$ & $\begin{array}{l}\text { Spray valve's } \\
\text { position/Primary } \\
\text { pression }\end{array}$ & Primary pressure & $\begin{array}{l}\text { Spray valve's } \\
\text { position/Primary } \\
\text { pression }\end{array}$ & $\begin{array}{c}\text { Spray valve's } \\
\text { position/Primary } \\
\text { pression }\end{array}$ & Primary pressure \\
\hline Pressurizer's heater & $\begin{array}{l}\text { valves } \\
\text { control }\end{array}$ & Primary pressure & Primary pressure & Primary pressure & Primary pressure & Primary pressure & Primary pressure & Primary pressure \\
\hline \multirow{2}{*}{ Main feedwater } & $\begin{array}{l}\text { main valves } \\
\text { main valves control }\end{array}$ & - & - & $\begin{array}{l}\text { Main feedwater's } \\
\text { flow }\end{array}$ & $\begin{array}{l}\text { Main feedwater's } \\
\text { flow }\end{array}$ & $\begin{array}{c}\text { Main feedwater's } \\
\text { flow }\end{array}$ & $\begin{array}{l}\text { Main feedwater's } \\
\text { flow }\end{array}$ & $\begin{array}{l}\text { Main feedwater's } \\
\text { flow }\end{array}$ \\
\hline & $\begin{array}{l}\text { bypass valves } \\
\text { bypass valves control }\end{array}$ & - & - & - & - & - & - & - \\
\hline \multirow{3}{*}{ Auxiliary feedwater } & turbopump control & - & . & $\begin{array}{l}\text { Turbine driven pump } \\
\text { speed }\end{array}$ & $\begin{array}{l}\text { Turbine driven pump } \\
\text { speed }\end{array}$ & $\begin{array}{l}\text { Turbine driven pump } \\
\text { speed }\end{array}$ & $\begin{array}{l}\text { Turbine driven pump } \\
\text { speed }\end{array}$ & $\begin{array}{l}\text { Turbine driven pump } \\
\text { speed }\end{array}$ \\
\hline & $\begin{array}{l}\text { turbine driven pump's valve } \\
\text { control }\end{array}$ & $\begin{array}{l}\text { Auxiliary feedwater's } \\
\text { flow }\end{array}$ & $\begin{array}{l}\text { Auxiliary feedwrater's } \\
\text { flow }\end{array}$ & - & - & - & - & - \\
\hline & $\begin{array}{l}\text { pump valves } \\
\text { control }\end{array}$ & $\begin{array}{l}\text { Auxiliary feedwater's } \\
\text { flow }\end{array}$ & $\begin{array}{l}\text { Auxiliary feedwater's } \\
\text { flow }\end{array}$ & - & - & . & - & . \\
\hline $\begin{array}{l}\text { High pressure injection } \\
\text { system }\end{array}$ & control & - & - & - & - & - & - & - \\
\hline $\begin{array}{l}\text { Low pressure injection } \\
\text { system }\end{array}$ & control & . & . & - & - & . & . & . \\
\hline Steam dury & control & Average temperature & Average temperature & Average temperature & Average temperature & Average temperature & Average temperature & Average temperature \\
\hline Turbine & & - & - & $\begin{array}{l}\text { Turbine's valve } \\
\text { position }\end{array}$ & - & $\begin{array}{c}\text { Turbine's valve } \\
\text { position }\end{array}$ & $\begin{array}{c}\text { Turbine's valve } \\
\text { position }\end{array}$ & - \\
\hline Control rod system & control & . & . & . & . & . & . & Control rod insertion \\
\hline
\end{tabular}

Fig. 5. Qualification matrix

\section{* transient 2 *}

Reactor trip due to

low SG level

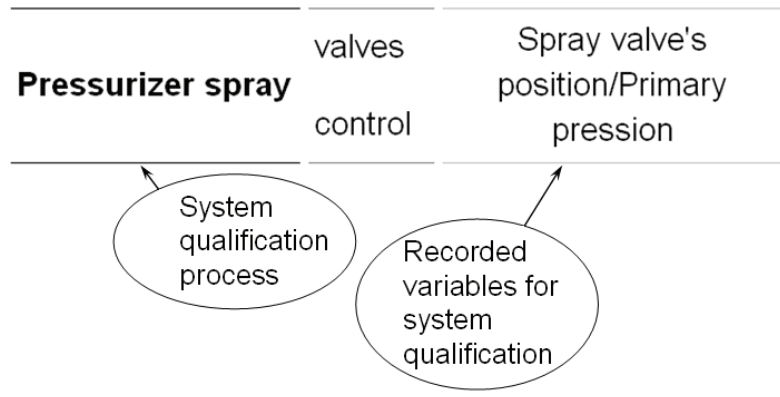

Fig. 6. Detailed box of the qualification matrix 
The matrix is also helpful for new studies. The analyst, knowing the relevant aspects of the scenario to be studied, could easily check in the table if the model used is properly qualified. The matrix can also be easily enhanced either by adding columns related to new suitable transients or adding new lines related to systems needed for qualification.

\section{Example cases}

This section briefly presents some relevant results of one example of analysis performed along with the concise description of other two cases. All the considered cases are related to actual situations in which the scenarios were studied by analysts using thermal-hydraulic codes and prepared nodalizations.

The example presented is the analysis of a reactor trip operating event due to high variation of neutron flux occurred in the Vandellòs-II NPP. More detail can be found in (Reventós 2010).

The transient was initiated by an electrical grid disturbance due to a storm, which caused disconnection of the main output switch, while the in-site electrical equipment switch remained connected. The plant therefore started operating on auto-consumption. Due to the loss of off-site power, the reactor and the turbine tripped and natural circulation was established. Later on, off-site power was recovered and operators brought the plant to Hot Zero Power (HZP).

The initial phase started with the loss of off-site power and lasted until the reactor trip. The sequence of events that caused the shutdown of the reactor lasted less than 1.0 second and is not easily studied mainly because of the short time of occurrence and also because of the relatively high time step of the collected time-trends. The post-trip event list did not help much.

The only symptom that pointed to a credible explanation was related to some primary flow data recorded by plant instrumentation. These data revealed that in 2.0 seconds the primary flow increased by about 4 or $5 \%$.

Since the plant was on auto-consumption, the electrical frequency could have increased and could have resulted in the subsequent increase of the Reactor Coolant Pump (RCP) speed. This suspicion needed to be confirmed. A calculation was performed in order to corroborate this hypothesis.

In order to approximate their real behaviour, different values of RCP speed were introduced in the BE model as a boundary condition until the primary flow increased by about 4 or $5 \%$, as had been observed in the plant. This flow increase produced a decrease in moderator temperature that could not be measured by usual temperature instruments (Figure 7) in the first second after the initiating event.

A calculation, using an Integral Plant Model, produced the evolution of temperature node by node for the whole core. Results were analyzed for all nodes and the temperature corresponding to the central node is shown in Figure 8. This decrease in core temperature produced an increase in power due to the effect of moderator temperature (Figure 9). This figure shows the power increase until the inflection due to the beginning of rod insertion at 0.6 seconds and the full decrease of power after time $=1.0$ second as an effect of the negative reactivity introduced. It must be pointed out that the time of insertion is about 1.5 seconds which is consistent with the power time trend. 


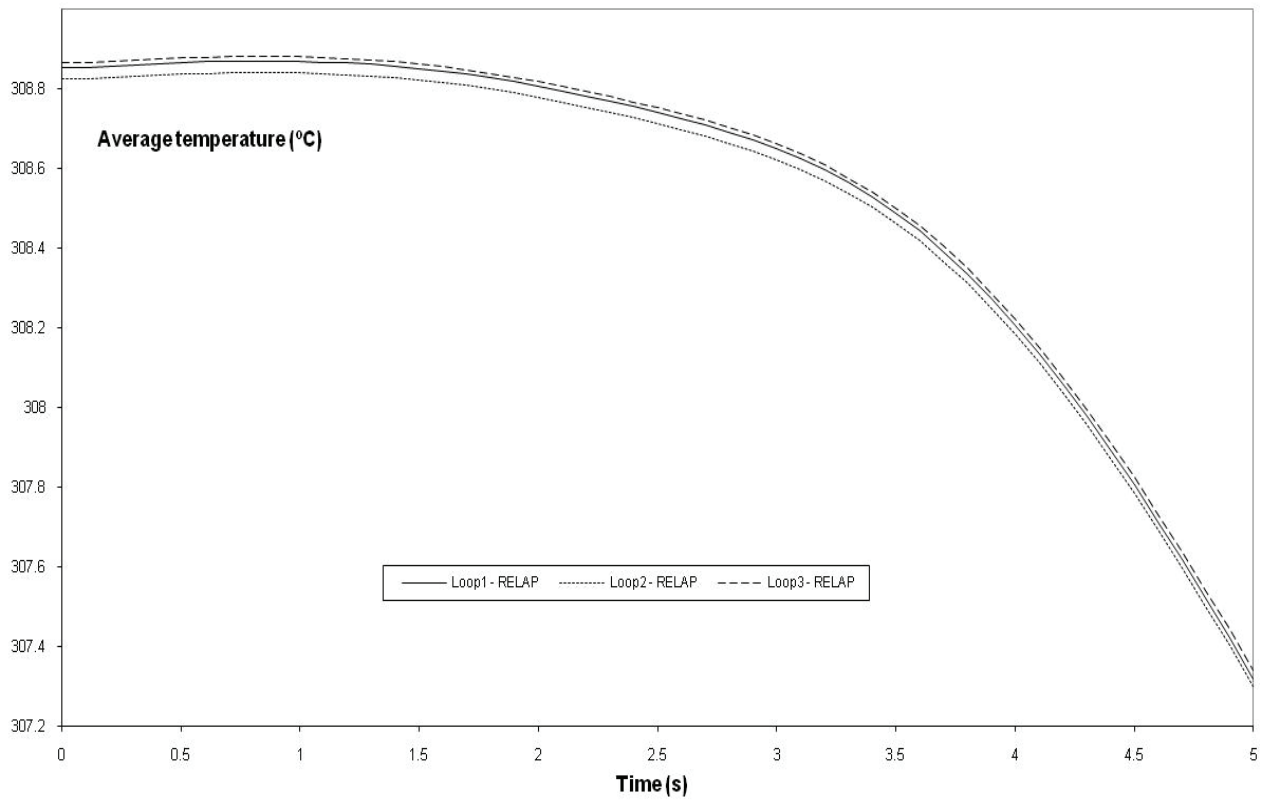

Fig. 7. Calculated average temperature

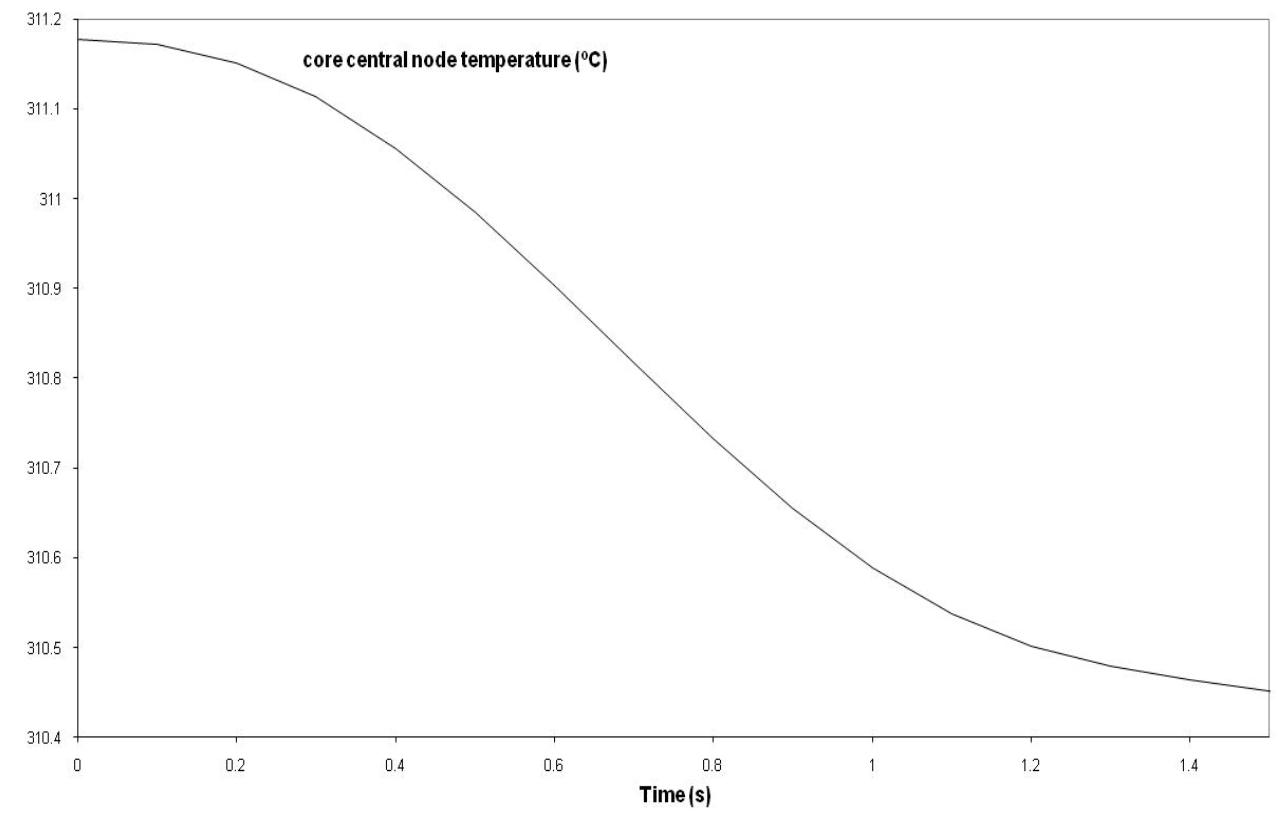

Fig. 8. Calculated temperature of core central node 


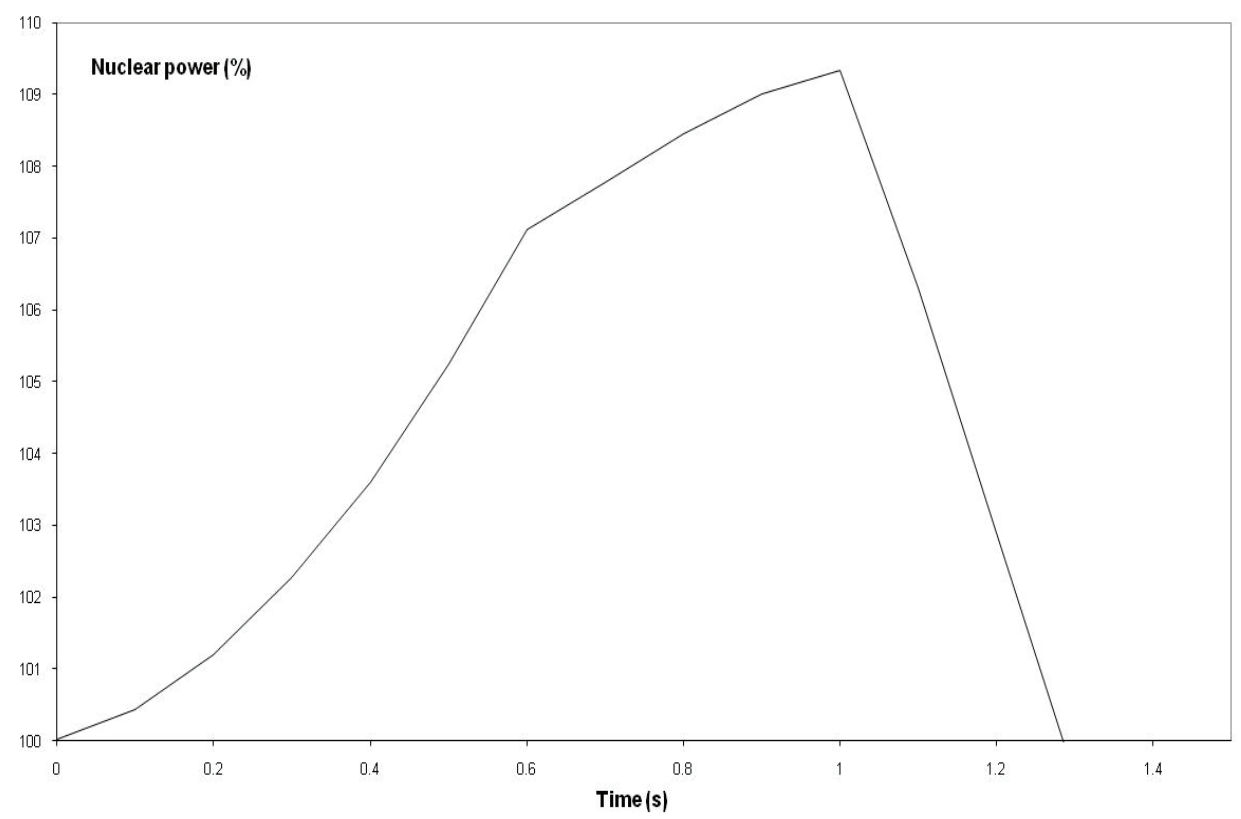

Fig. 9. Calculated nuclear power

Although the neutronics of the Integral Plant Model used, is usually not very detailed (it is a point-kinetics model), it is capable of predicting the increase in reactor power due to moderator temperature decrease and, as a consequence, the reactor trip due to high variation of neutron flux.

The model has been extremely useful in clarifying the sequence of events corresponding to the transient that actually occurred in the plant on December 2, 1991. The study provided an answer to the concerns of the person responsible for operation related to the scenario and was also helpful to identify the inadequacy of thermocouple location as a design deficiency. This identification was a necessary step previous to its replacement that took place soon after the event.

The analysis is a good example of how integral plant models can result in a real benefit as part of the support activities to the operation of a nuclear power plant.

The transient was included in the Qualification Matrix and it is currently being re-analyzed after any major change performed in the model.

Operating events, as the one depicted above, are maybe the most significant analyses performed for support of plant operation. Other cases are analyzed. Two concise descriptions are presented below.

Some analyses are performed, as the one that follows, in strong connection to both EOP and PSA. Studies like this one are maybe not the more significant but they are for sure the most usual. The studied sequence consists in a total loss of Feed Water (FW).

EOP/PSA transient analyses are traditionally performed using Integral Plant Models. Results were a successful first approach to operation support and the study was carried out following some concern of the person responsible of plant operation. More detail on the analysis can be found in (Reventós, 2007b). 
After the total loss of FW takes place, heat transfer from the primary to the secondary side degrades and causes a decrease of the SG level. Once this symptom has been detected, the procedure starts by opening 1 PORV and actuating 1 HPIS train. Water injected into the primary system at low temperature is heated by decay power and comes out through the relief valve. The procedure results in a pressure decrease which means that energy produced is completely extracted.

The base case brings the plant to a safe situation without violating design limits as hot rod clad temperatures show a general decreasing trend during the whole transient. The calculation properly captures the main relevant thermal-hydraulic features of the scenario.

Once the base case was successfully simulated, a strategy was defined to answer the following questions:

- Impact of PORV and HPIS partial availability (less than 2 PORV or 2 HPIS trains)

- Maximum time to start the procedure after the level symptom occurs

- Relevant heat sink recovery phenomena (although recovery actions are quite fast, they involve different components and need some time)

The answers to the questions were obtained and the operation team got a better general picture of the scenario and related phenomena. As obviously each answer has an impact on the others, the strategy applied was to launch quite a large number of combined scenarios in order to cover different situations that could potentially occur. For a given combination of component availability, a series of different procedure starting times have been tried and for each of these calculations heat sink recovery was also imposed at different times. The total number of cases was 61 . In this case the complete set of calculations was performed by the analyst.

The next concise description presented in the current context is not related to transient analysis but to slow degradation of a very significant component: the steam generators of a PWR plant. The study was carried out for Ascó NPP.

Due to some problems related to the material used in manufacturing steam generator tubes, degradation was taking place and the probability of having a tube rupture was increasing from cycle to cycle. To face the problem the team giving support to plant operation, started with different engineering actions, most of them were design modifications related to the chemistry of the secondary circuit devoted to replace materials that were supposed to power corrosion. At each reload an Eddy current extensive inspection was carried out in order to quantify the degradation and as a consequence to make a decision on which tubes needed to be plugged. The problem was quite serious because in few years the number of plugged tubes increased at an important rate.

Using the Integral Plant Model of Ascó NPP, an analysis was carried out. The results obtained became interesting information to help decision making. The work done faced both realistic modelling of actual situations and predictive simulation of eventual future plugging.

After each reload and following the actual tube plugging, the plant model was adjusted with realistic criterion. The specific development of the model was to decrease heat exchange surface from primary to secondary side and also to reduce the primary flow area following the actual plugging. The model stabilized at a slightly different working point. Maybe the more interesting parameters to check were the secondary pressure and the stabilized position of turbine valve. Such stable values of Pressure and valve position are shown in Figure 10 along with model predictions as a function of plugging percentage. Checking and comparing such parameters provided additional validation for the specific situation. The validated model could then be used for the usual purposes maintaining its accuracy. 


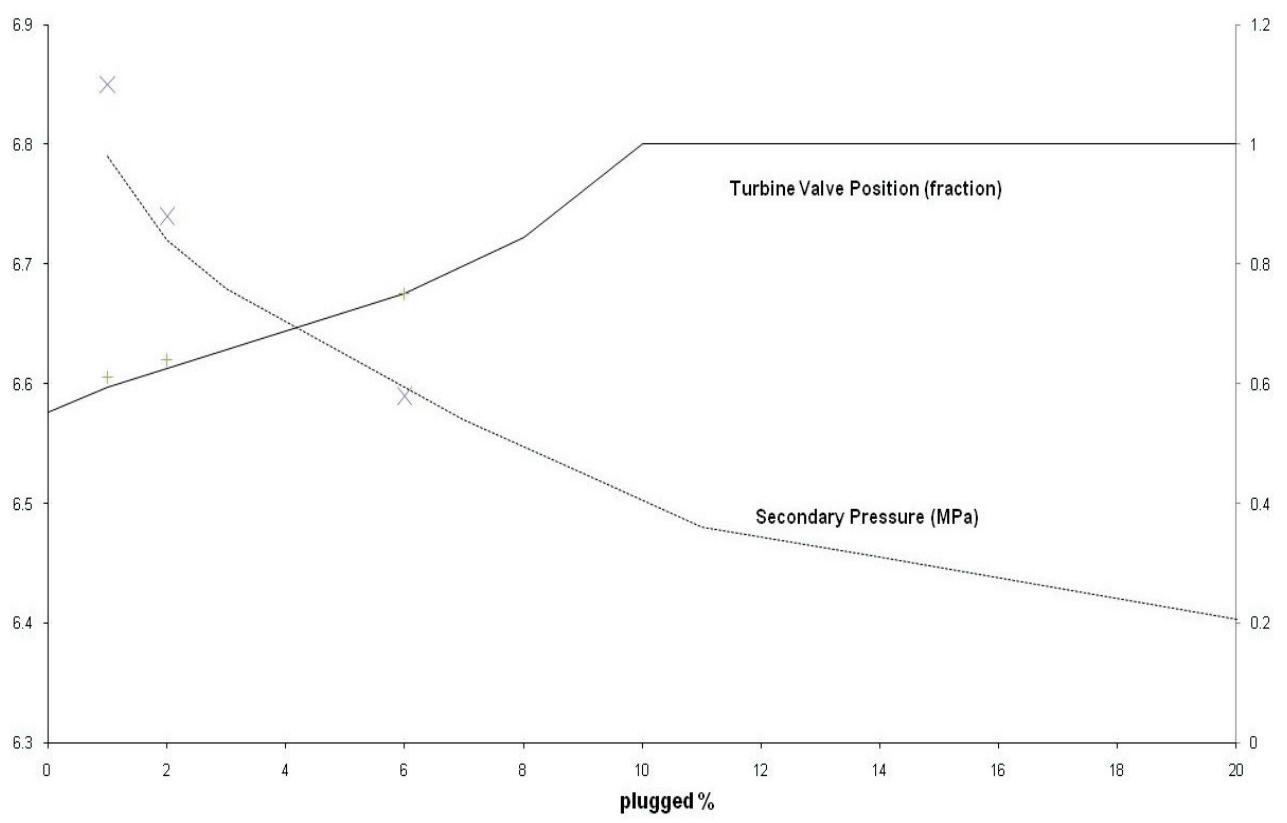

Fig. 10. Secondary pressure and turbine valve position vs. SG tube plugged percentage

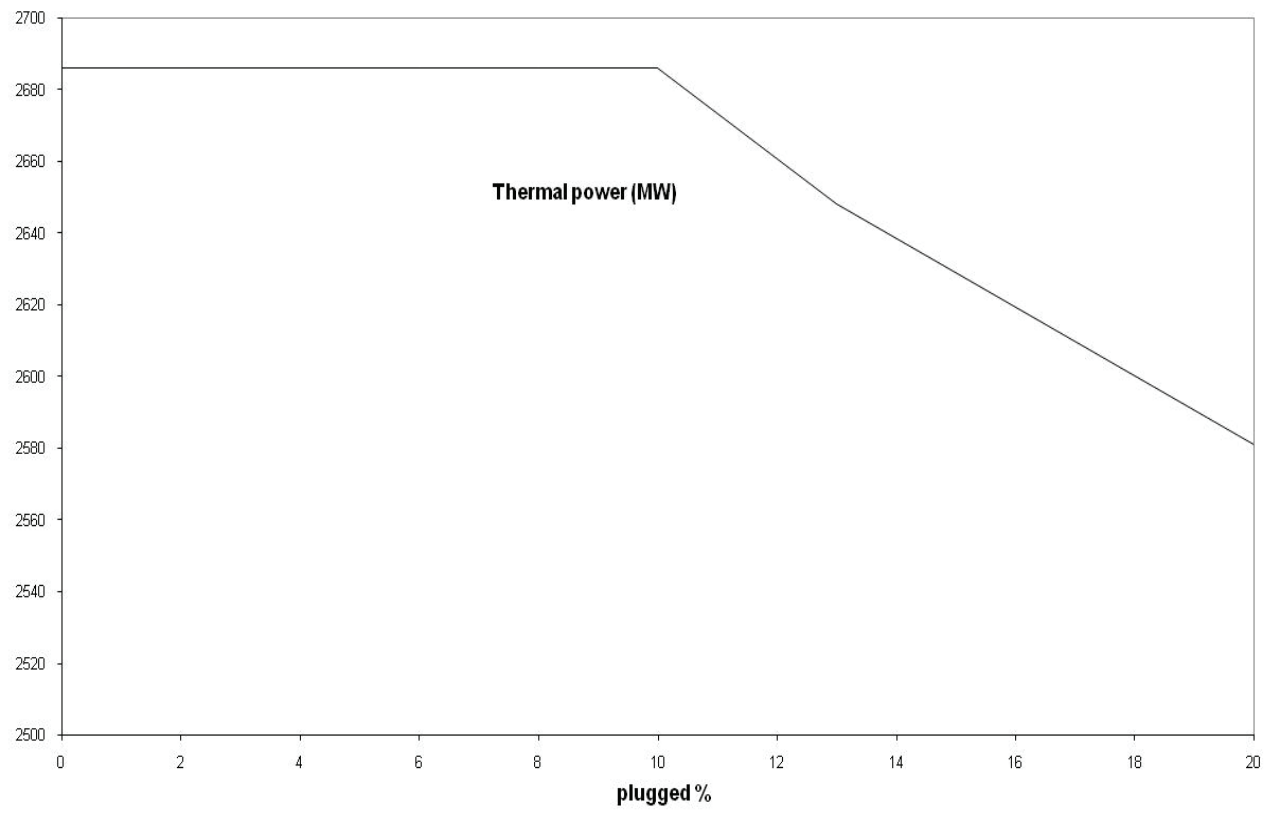

Fig. 11. Thermal power vs. SG tube plugged percentage 
The predictive simulation of eventual future plugging was even more interesting. Symmetric and asymmetric configurations were modelled at an increasing plugging percentage and key parameters were evaluated. When plugging percentage increased secondary pressure decreased and turbine valve stabilized at a wider position to compensate and allow the nominal value of steam mass flow (see Figure 10). Once turbine valve at certain plugging percentage reached the fully wide open position the secondary system stopped being able to extract all the thermal power produced. As shown in Figure 11 , higher plugging percentages resulted in a thermal power smaller than the nominal one.

The predictive simulation gave quite clear results about 3 o 4 cycles (at that time Ascó follow 12 months cycles) before the eventual decrease of thermal power. The results of this analysis, along with other technical studies, became extremely helpful for making the decision of steam generator replacement. The decision was made on time and the replacement was carried out successfully.

\section{Conclusions}

This chapter has shown the relevance of thermal-hydraulic analysis devoted to give support to plant operation. Integral Plant Models prepared using system codes, and properly qualified, are a valuable tool to carry out the studies presented.

It has also been shown the significance of tasks to be performed by the so called thermalhydraulic analyst supporting plant operation. If this analyst belongs to the technical team that takes care of engineering plant support, his studies become more effective.

Taking care of plant models and personally performing at least the first approach analysis of any of the issues involved, is a suitable strategy. Depending on the amount of work needed for each specific analysis, the whole work or only a part of it is done by him. Benefits are clear in both cases.

The examples presented or briefly described illustrate the job of performing termalhydraulic calculations as a first approach of the analysis of plant dynamic behaviour.

\section{Acknowledgement}

The examples presented in this chapter are related to the NPPs of Ascó and Vandellòs-II operated by ANAV. The author is grateful to the management and the staff of the ANAV for their consent to this publication.

\section{References}

Ashley R., El-Shanawany M., Eltawila F., D’Auria F., November 1998. Good Practices for User Effect Reduction. NEA/CSNI/R(98)22.

Berthon A., Petruzzi A., del Nevo A., Reventós F., September 2005. Consistent code qualification process and application to the LOBI test BL-44. IAEA Technical Meeting on: Use of a Best Estimate Approach in Licensing with Evaluation of Uncertainties; Pisa, September 2005

IAEA, 2002. Accident Analysis for Nuclear Power Plants. Safety Report Series No. 23. International Atomic Energy Agency. Vienna.

IAEA Safety Reports Series No 48, 2006. Devepolment and Review of Plant Specific Emergency Operating Procedures. 
Llopis C., Casals A., Pérez J., Mendizábal R., December 1993. Assessment of RELAP5/MOD2 against a main feed water turbo pump trip transient in the Vandellos-II NPP. International Agreement Report. Nureg/ia-110

Llopis C., Pérez J., Casals A., Mendizábal R., May 1993. Assessment of RELAP5/MOD2 against a $10 \%$ load rejection transient from $75 \%$ steady state in the Vandellòs-II NPP. International Agreement Report. Nureg/ia-109

Llopis C., Reventós F., Batet L., Pretel C., Sol I., March 2007. Analysis of Low Load Transients for the Vandellòs-II NPP. Application to Operation and Control Support. Nuclear Engineering and Design, 237 (2007) 2014-2023

Martínez V., Reventós F., Pretel C., Sol I.; Code Validation and Scaling of the ROSA/LSTF Test 3-1 Experiment; Topsafe 2008 - European Nuclear Society; Dubrovnik, Croatia, September-October 2008

A. Petruzzi, F. D'Auria, W. Giannotti, "Description of the procedure to qualify the nodalization and to analyze the code results", May, 2005.

Posada J., Martín M., Reventós F., Llopis C., September 1997. Interactive graphical analyser based on RELAP5/MOD3.2-NPA. 2nd CSNI specialist meeting on simulators and plant analysers. Espoo-Finland

Reventós F., Batet L., Llopis C., Pretel C., Salvat M., Sol I.; Advanced qualification process of ANAV NPP integral dynamic models for supporting plant operation and control; Nuclear Engineering and Design 237 (1) 54-63; 2007

Reventós F., Batet L., Llopis C., Pretel C., Sol I.; Thermal-Hydraulic Analysis Tasks for ANAV NPPs in Support of Plant Operation and Control; Science and Technology of Nuclear Installations, Volume 2008, Article ID 153858, 13 pages

Reventós F., Llopis C., Batet L., Pretel C., Sol I.; Analysis of an actual reactor trip operating event due to a high variation of neutron flux occurring in the Vandellòs-II nuclear power plant; Nuclear Engineering and Design 240 (2010) 2999-3008

Reventós F., Batet L., Pretel C., Ríos M., Sol I., March 2007. Analysis of the Feed \& Bleed procedure for the Ascó NPP. First approach study for operation support. Nuclear Engineering and Design, 237 (2007) 2006-2013

Reventós F., Batet L., Pretel C., Llombart O., Sol I., Romera S., September 2006. Improving PSA Usefulness Using the Results of Thermalhydraulic Best Estimate Models of ANAV Reactors. Working Material. IAEA Technical Meeting. Effective Integration of Deterministic and Probabilistic Safety Analysis in Plant Safety Management. Barcelona (Spain)

Reventós F., Sánchez-Baptista J., Pérez-Navas A., Moreno P., December 1993. Assessment of a pressurizer spray valve faulty opening transient at Ascó nuclear power plant with RELAP5/MOD2. International Agreement Report. Nureg/ia-121.

Reventós F., Posada J., Llopis C., Pretel C., Moreno P., April 2001. Improving NPP availability using thermal hydraulic integral plant models. assessment and application of turbine runback scenarios. Proceedings. 9th International Conference on Nuclear Engineeringicone9. 


\title{
A Literature Survey of Neutronics and Thermal-Hydraulics Codes for Investigating Reactor Core Parameters; Artificial Neural Networks as the VVER-1000 Core Predictor
}

\author{
Farshad Faghihi ${ }^{1}$ H. Khalafi ${ }^{2}$ and S. M. Mirvakili ${ }^{1}$ \\ 1Shiraz University, School of Mechanical Engineering, \\ Department of Nuclear Engineering, Shiraz, \\ ${ }^{2}$ Reactors and Accelerators Research and Development School, \\ Nuclear Science and Technology Research Institute (NSTRI), \\ Atomic Energy Organization of Iran (AEOI), Tehran 14399-51113,
}

Iran

\section{Introduction}

In this chapter, we investigated an appropriate way to predict neutronics and thermalhydraulics parameters in a large scale VVER type nuclear reactors. A computer program is developed to automate this procedure using Artificial Neural Network (ANN) method. The neutronics and thermal-hydraulics codes are connected to each other and then the neural network method use results with different configuration of a suggested core for prediction. The main objective of this research is to develop fast and first estimation tool (a software) based on ANNs which allows large explorations of core safety parameters. This tool is very useful in reactor core design and in-core fuel management or loading pattern optimization. Therefore, herein, an overview study on the multiphysics/multiscale coupling methods for designing current and innovative VVER systems by coupling neutronics parameters (using MCNP 5) and thermal-hydraulics simulator (e.g., COBRA-EN) are carried out. This work is aimed to extend the modeling capabilities of coupled Monte Carlo/Subchannel codes for whole core simulations based on pin-level in order to address many problems e.g. higher burn-up, Mox-fuels, or to improve the performances and accuracy of reactor dynamics.

Verification and validation of the above development are the main concern and important procedures and therefore taking into account using experimental data or another code-tocode benchmarking. Finally the extended simulation capabilities should be applied to analyze a selected VVER reactor and we present our input computer codes for interested readers. Also, our future designed user friendly Artificial Neural Network (ANN) software would be given for everyone who wants to get it.

Bushehr Nuclear Power Plant (BNNP), a VVER-1000 Russian model, was simulated during the first plant operational period using WIMS and CITATION codes (Faghihi et al., 2007). Modelling of all rods (including fuel rods, control rods, burnable and non-burnable poison rods) and channels (including central guide channel, measuring channel) were carried out 
using the WIMS code. Moreover, modelling of the fuel assemblies and reactor core is completed using the CITATION code. The multi-group constants generated by WIMS for different fuel configurations are fed into CITATION. In our past mentioned article, average burn ups and calculated reactivity coefficients from Beginning of Cycle (BOC) to Middle of Cycle (MOC) for the VVER-1000 BNPP were carried out. A thermal-hyraulics analysis for the VVER-1000 were employed using COBRA-EN code (Mirvakili et al., 2010). Moreover, a $13 \times 13$ square array fuel assembly core of a 1000 MWe Westinghouse reactor was investigated and its "Prompt reactivity coefficient", which is an important factor in the study of nuclear power excursions, and also "power coefficient of reactivity" were calculated using MCNP-5 (Faghihi and saidinezhad, 2011). MCNP 5 has seven new features with respect to the older ones and complete description along with a list of bug fixes are listed in its release notes (MCNP-5 Team, 2008).

In the incoming sections we will investigate ANNs theory and then we introduce with MCNP and COBRA-EN code as our toolboxes for the reactor neutronics and thermal hydraulics feedbacks

\section{Neutronics codes}

A literature review of available coupled codes for application to LWR's analysis has been conducted to investigate their capabilities for VVER applications. In order to identify the types of codes coupled, different types of methods implemented into neutronics codes and thermal-hydraulics codes need to be discussed. The neutron flux can be predicted with diffusion codes, deterministic codes and Monte Carlo methods.

\subsection{Diffusion and transport codes}

Diffusion codes solve the neutron diffusion equation to obtain the neutron flux, from which the power distribution is computed. They use macroscopic cross section data for neutron particles, processed usually from two or more energy groups. The modeling of a reactor core or fuel assembly is homogenized for the diffusion approximations to be valid. Diffusion codes have been well suited to analyze reactors, which are designed with relatively homogeneous distributions of fuel, moderator and absorber materials. However, with higher heterogeneity such as in the VVER or HPLWR (High-Performance Light-Water Reactor) fuel assembly, the simplified model will produce inaccurate results. Details of the VVER fuel assembly such as coolant density in different sub-channels and power distribution of different fuel rods, which are needed in the coupling, cannot be obtained.

Until now, coupling experience for PWR and BWR reactor have been with diffusion codes coupled with system codes, which have been applied for various transient analysis. For transient analysis diffusion codes and system codes are restricted to simplified geometries and their application cannot be extended to complex geometries such as for fuel assembly design of a VVER. The neutronic code PARCS (Purdue Advanced Reactor Core Simulator) developed at the Purdue University is used to predict the dynamic response of the reactor to reactivity perturbations such as control rod movement or change in temperature/fluid conditions in the reactor core. A coupling interlace of PARCS with TRAC-M, a system code, was completed by Miller et al. The coupled code was tested using the OECD PWR main steam line break (MSLB). The coupled TRAC-M/PARCS was also applied for turbine trip (TT) transient analysis of the OECD/NRC BWR by Lee et al. The PARCS code has also been 
coupled with the system code RELAP5 for analysis of the peach bottom turbine trip (TT), Salah et al.

DYN3D is a neutron kinetic code developed to investigate reactivity transients in the reactor core with hexagonal or quadratic fuel assembly. The neutron diffusion equations are solved for two groups. An internal coupling approach of DYN3D with ATHLET is a system code that has been developed by Grundmann et al. The coupled code DYN3D/ATHLET has been applied for analysis of BWR TT transient.

The SKETCH-N code solves neutron diffusion equation in $x-y-z$ geometry for steady state and neutron kinetic problems. The code treats an arbitrary number of neutron energy group and delayed neutron precursors. The SKETCH code has been implemented into thermalhydraulic code TRAC for analysis of rod injection transients Asaka et al.

NEM (Nodal Expansion Method) is a 3-D multi-group nodal code developed and used at the Pennsylvania State University for modeling both steady state and transient core conditions. The code has options for modeling 3-D Cartesian, cylindrical and hexagonal geometry. NEM has been coupled to the system code TRAC-PF for MSLB transient analysis of a PWR Ivanov et al. and Ziabletsev et al. and for BWR core transient, NEM has been coupled with TRAC-BFI by Fu et al.

NESTLE is a multi-dimensional neutron kinetic code developed at the North Carolina State University. It solves the two group or four group neutron diffusion equations in Cartesian or hexagonal geometry.

QUABOX is a neutron kinetic code developed in the 70s at GRS in Germany for 3-D core neutron flux and power calculations in steady state and transient conditions. It solves the two-group neutron energy diffusion equation through local polynomial approximation of the neutron flux. The QUABOX code has been coupled with ATHLET internally for analysis of the OECD/NRC BWR turbine trip benchmark by Langenbuch et al. A serial coupling is applied. The T-H code ATHLET makes the first calculation step and when it is finished the core model QUABOX/CUBBOX calculates the same step for the neutronics on the same computer.

PANBOX is a three dimensional neutron kinetics code coupled with a multidimensional core thermal-hydraulics module, developed to perform PWR safety analysis and transients in which power distribution is significantly affected. The time-dependent few-group diffusion equation is solved in Cartesian geometry using a semi-analytical nodal expansion method (NEM). The PANBOX code system has been coupled with the thermal-hydraulics system code RELAP for analysis of the OECD/NEA PWR MSLB, Sanchez-Espinoza et al. Verification of the coupled PANBOX / RELAP was performed by Jackson et al. for core transient analysis.

CITATION code is a three dimensional, multi group diffusion code (Oak Ridge National Laboratory, 1972), is used for core simulation. This code was designed to solve problems involving the finite-difference representation of diffusion theory treating up to three space dimensions with arbitrary group-to-group scattering. Explicitly, finite-difference approximation in space and time has been implemented. The neutron flux-eigenvalue problems are solved by direct iteration to determine the multiplication factor or the nuclide densities required for a critical system. In the input file of this code the macroscopic crosssections are required which are prepared by running WIMSD-4 code (Winfrith, 1982). As this code uses the transport theory in its calculations, the results have a high degree of accuracy. 


\subsection{Discrete-ordinate codes}

Deterministic codes are most commonly based on the discrete ordinates method. They solve the Boltzmann transport equation for the average particle behavior to calculate the neutron flux. With discrete ordinate methods, the phase space is divided into many small boxes and particles are moved from one box to another. If this approach is to be used for modeling a VVER fuel assembly, the guide tubes, the coolant sub-channels, and fuel rods will be homogenized and the medium is discretized to solve the transport equation. This type of geometry modeling will not accurately represent the important design details essential for the VVER fuel assembly. Deterministic codes use macroscopic cross section data, which are processed from multi-group energies. Processed macroscopic cross section data from microscopic scale are required for different parts in the geometry. For complicated geometries with varying parameters such as coolant and moderator density, preparation of the macroscopic cross section data would also require a lot effort. Therefore deterministic codes need to be homogenized for complex geometries. The global solutions are obtained with truncated errors. Computer codes based on deterministic methods include DORT, two dimensional (X-Y, and R-Z) geometries, TORT, a three-dimensional discrete transport code DORT-TD, a transient neutron transport code, KARPOS, a modular system code developed by Broeders et al.

\subsection{Monte-Carlo method}

A Monte Carlo method does not solve an explicit equation like the deterministic code, but rather obtains the answers by simulating individual particles and recording some aspects (tallies) of their average behavior. Monte Carlo codes use a continuous energy scale to represent the variation of cross section data. They are widely used because of the capability of complex geometries modeling and accurate solution produced with the continuous energy scale used to represent the cross section data. Computer codes based on the MonteCarlo methods include: MCNP (Monte Carlo N-Particle) is a general-purpose, continuousenergy, generalized-geometry, coupled neutron/photon/electron transport code. The MCNP code for neutronics analysis is described by Briesmesiter, 2000 from the Los Alamos National laboratory. Different versions of MCNP have been developed, for example MCNP4C for low energy calculation and MCNPX for higher energies. The application of the Monte Carlo codes in nuclear energy is increasing for fuel assembly and core design analysis typically in BWR where the density varies in the core. Mori et al. has already coupled the Monte-Carlo MCNP has been successfully coupled with a thermal-hydraulics system code for power and reactivity analysis of a supercritical fast reactor (SCFR) core that does not include moderator tubes, hence a simplified design.

\section{Thermal-hydraulics codes}

\subsection{System codes or lumped approach}

System codes are based on a lumped parameter approach. This means, for nuclear power plant (NPP) application the components in the primary and secondary system are represented by a one-dimensional model. Details of a fuel assembly such as moderator rod, individual sub-channels for density variation study cannot be revealed through such means. The basic equations for continuity, momentum and energy are applied and averaged and the thermal-hydraulics properties for each component are obtained. The smallest volume is typically a total core or major parts of it. System codes are commonly used in LWR 
application for different types of transient and safety analysis. Widely used system codes include:

ATHLET, (Analysis of Thermal-hydraulics of LEaks Transient) has been developed by the Gesellschaft für Reaktorsicherheit (GRS) for analysis of anticipated and abnormal plant transients, small and intermediate leaks and large breaks in light water reactors. The concept of ATHLET for analysis of PWR and BWR system has been described by Burwell et al. The ATHLET code has been coupled with the 3-D core model neutronic code DYN3D for analysis of BWR turbine trip benchmark, Grundmann et al. Validation of the ATHLET thermal-hydraulics code for PWR and BWR was presented by Glaeser. The coupling interlace of ATHLET with the neutronic core model DYN3D has been reported by Langenbuch et al. The coupled code ATHLET- QUABOX/CUBBOX has been used by Langenbuch et al. for analysis of the OECD/NRC BWR turbine trip benchmark.

RELAP (Reactor Excursion and Leak Analysis Program) is used for transient simulation of LWRs. It is widely used for LWR transient analysis in PWR and BWR. The RELAP5 code has been coupled with point kinetic code for analysis of OCED/NEA PWR MSLB by SanchezEspanioza and Nigro et al. Bovalini et al. reported coupled application of RELAP and comparison with different codes for TMI-MSLB. The CATHARE code is used for transient analysis of PWR plants, VVER and BWR. The CATHARE code has been coupled with CRONOS2-FLICA4 for BWR turbine trip analysis Mignot et al.

\subsection{Sub-channel codes}

Sub-channel codes are used for multi-component modeling in the core. A core is represented by the sub-assemblies and the sub-assembly by different sub-channels and other water channels and fuel rods. The basic equations are solved for control volumes in the scale of sub-channels. The sub-channel codes are capable of three-dimensional geometry modeling. Codes that are based on this approach include:

COBRA (Coolant Boiling in Rod Arrays) is a public computer code used for thermalhydraulics analysis with implicit cross-flow between adjacent sub-channels, single flow and homogeneous two-phase fluids. It is used world-wide for DNBR (departure from nucleate boiling ratio) analysis in LWR sub-channels as well as for 3-D whole PWR core simulation with one or more channels per fuel assembly, Wheeler et al.

MATRA (Multi-channel Analyzer for steady states and Transient in Rod Arrays) is a subchannel analysis code developed at KAERI (Korea Atomic Energy Research Institute), Yoo et al. The main concept of the MATRA code is based on COBRA.

The STAFAS (Sub-channel Thermal-hydraulics Analysis of Fuel Assembly under Supercritical Conditions) code was developed by Cheng et al. It is based on the concept of the COBRA code but includes special features of the HPLWR such as: downward flow of the moderator water and incorporates steam table that allows the prediction of supercritical water properties. The code is flexible and allows for complex geometry modeling. Heat transfer from solid surfaces can be easily implemented. The present version of the STAFAS code is for steady state conditions and single-phase flow only.

FLICA-4 is a thermo-hydraulic code developed at the French Atomic Energy Commission (CEA) for computing three-dimensional, transient or steady, two-phase flows in nuclear reactors. The code is described in the paper by Allaire for 3-D transient computation. The FLICA code has been coupled with the system code CATHARE and CRONOS2, a 3D neutronics code for computation of a BWR turbine trip, Mignot et al. 


\begin{tabular}{|c|c|c|c|c|}
\hline No. & Title and authors & Coupled codes & NPP & $\begin{array}{l}\text { Transient } \\
\text { type ef. }\end{array}$ \\
\hline \multirow{3}{*}{1} & \multirow{3}{*}{$\begin{array}{l}\text { Coupling of the Thermal-hydraulics } \\
\text { Code TRAC } \\
\text { with 3-D Neutron Kinetics Code SKETCH-N } \\
\text { H. Asaka, V.G. Zimin, } \\
\text { T. Iguchi, Y. Anoda }\end{array}$} & \multirow{3}{*}{$\begin{array}{l}\text { J-TRAC } \\
\text { TRAC-BF1 } \\
\text { Sketch-N }\end{array}$} & PWR & RIA \\
\hline & & & $\begin{array}{c}\text { BWR } \\
\text { (Ringhals-1) }\end{array}$ & $\begin{array}{l}\text { Stability } \\
\text { benchmark } \\
\text { cases }\end{array}$ \\
\hline & & & BWR & Instabilities \\
\hline 2 & $\begin{array}{l}\text { Core-wide DNBR Calculation } \\
\text { for NPP Krško MSLB } \\
\text { I-A. Jurkoviá, D. Grgiá, N. Debrecin }\end{array}$ & $\begin{array}{c}\text { RELAP5/MOD3.2 } \\
\text { COBRA III C } \\
\text { QUABOX/CUBBOX } \\
\end{array}$ & $\begin{array}{c}\text { PWR } \\
\text { (NPP Krško) }\end{array}$ & MSLB \\
\hline \multirow[b]{2}{*}{3} & \multirow{2}{*}{$\begin{array}{l}\text { MSLB Coupled 3-D } \\
\text { Neutronics/Thermal-hydraulics } \\
\text { Analysis of a Large PWR } \\
\text { Using RELAP5-3-D } \\
\text { F. D'Auria, A. Lo Nigro, } \\
\text { G. Saiu, A. Spadoni }\end{array}$} & \multirow[b]{2}{*}{$\begin{array}{c}\text { RELAP5/MOD3.2 } \\
\text { NESTLE }\end{array}$} & $\begin{array}{c}\text { PWR B\&W } \\
\text { TMI-1 }\end{array}$ & \multirow[b]{2}{*}{ MSLB } \\
\hline & & & AP-1000 & \\
\hline \multirow{3}{*}{4} & \multirow{3}{*}{$\begin{array}{l}\text { TMI-1 MSLB Coupled 3-D Neutronics/ } \\
\text { Thermal-hydraulics Analysis: } \\
\text { Application of RELAP5-3-D and Comparison } \\
\text { with Different Codes } \\
\text { R. Bovalini, F. D' Auria, } \\
\text { G.M. Galassi, A. Spadoni, Y. Hassan } \\
\end{array}$} & $\begin{array}{c}\text { RELAP5/MOD3.2.2 } \\
\text { PARCS } \\
\end{array}$ & \multirow{3}{*}{$\begin{array}{l}\text { PWR } \\
\text { (B\&W } \\
\text { TMI-1) }\end{array}$} & \multirow{3}{*}{ MSLB } \\
\hline & & $\begin{array}{c}\text { RELAP5/MOD3.2.2 } \\
\text { QUABBOX }\end{array}$ & & \\
\hline & & $\begin{array}{l}\text { RELAP5/3-D } \\
\text { NESTLE }\end{array}$ & & \\
\hline 5 & $\begin{array}{l}\text { PWR REA Sensitivity Analysis of } \\
\text { TRAC-PF1/NEM Coupling Schemes } \\
\text { N. Todorova, K. Ivanov }\end{array}$ & $\begin{array}{l}\text { TRAC-PF1 } \\
\text { NEM }\end{array}$ & $\begin{array}{c}\text { PWR (B\&W) } \\
\text { TM-1 }\end{array}$ & REA \\
\hline 6 & $\begin{array}{l}\text { Coupled 3-D Neutronic/Thermal-hydraulics } \\
\text { Codes Applied to } \\
\text { Peach Bottom Unit } 2 \\
\text { A. Ma Sánchez, G. Verdú , A. Gómez }\end{array}$ & & $\begin{array}{l}\text { BWR } \\
\text { (Peach } \\
\text { Bottom } \\
\text { Unit 2) } \\
\end{array}$ & TT \\
\hline 7 & $\begin{array}{l}\text { Study of the Asymmetric Steam Line Break } \\
\text { Problem by the Coupled Code System } \\
\text { KIKO3D/ATHLET } \\
\text { Gy. Hegyi, A. Keresztúri, I. Trosztel }\end{array}$ & $\begin{array}{l}\text { ATHLET } \\
\text { KIKO3D }\end{array}$ & VVER 440 & MSLB \\
\hline \multirow[b]{3}{*}{8} & \multirow{3}{*}{$\begin{array}{l}\text { Development of Coupled Systems of } \\
\text { 3-D Neutronics and Fluid-dynamic System } \\
\text { Codes and their Application } \\
\text { for Safety Analysis } \\
\text { S. Langenbuch, K. Velkov, } \\
\text { S. Kliem U. Rohde, M. Lizorkin, } \\
\text { G. Hegyi, A. Kereszturi }\end{array}$} & \multirow{3}{*}{$\begin{array}{l}\text { ATHLET } \\
\text { DYN3D } \\
\text { BIPR-8 }\end{array}$} & \multirow{3}{*}{ VVER-1000 } & LOFW \\
\hline & & & & \begin{tabular}{|c}
$\begin{array}{c}\text { Station black } \\
\text { out }\end{array}$ \\
\end{tabular} \\
\hline & & & & MCP stop \\
\hline 9 & $\begin{array}{l}\text { VIPRE-02 Subchannel Validation } \\
\text { Against NUPEC BWR } \\
\text { Void Fraction Data } \\
\text { Y. Aounallah, P. Coddington }\end{array}$ & VIPRE-02 ARROTTA & BWR & $\begin{array}{l}\text { Void } \\
\text { fraction } \\
\text { validation } \\
\text { study }\end{array}$ \\
\hline 10 & $\begin{array}{l}\text { High Local Power Densities Permissible } \\
\text { at Siemens Pressurised Water Reactors } \\
\text { K. Kuehnel, K.D. Richter, } \\
\text { G Drescher, I. Endrizzi }\end{array}$ & PANBOX & PWR & \begin{tabular}{|c|} 
Maximum \\
local heat \\
$\begin{array}{c}\text { flux investi- } \\
\text { gation }\end{array}$ \\
\end{tabular} \\
\hline
\end{tabular}

Table 1. Overview of 3-D coupled neutronics/thermal-hydraulics calculations available from the literature 


\begin{tabular}{|c|c|c|c|c|}
\hline No. & Title and authors & Coupled codes & NPP & $\begin{array}{l}\text { Transient } \\
\text { type ef. }\end{array}$ \\
\hline 11 & $\begin{array}{l}\text { Analysis of a Boron Dilution Accident for } \\
\text { VVER-440 } \\
\text { Combining the Use of the Codes DYN3D and } \\
\text { SiTap } \\
\text { U. Rohde, I. Elkin, V. Kalinenko }\end{array}$ & $\begin{array}{l}\text { SiTap } \\
\text { DYN3D }\end{array}$ & VVER 440 & RIA \\
\hline \multirow[t]{2}{*}{12} & \multirow{2}{*}{$\begin{array}{l}\text { RELAP5-PANTHER Coupled } \\
\text { Code Transient Analysis } \\
\text { B.J. Holmes, G.R. Kimber, } \\
\text { J.N. Lillington, M.R. Parkes }\end{array}$} & \multirow{2}{*}{$\begin{array}{l}\text { RELAP5 } \\
\text { PANTHER }\end{array}$} & \multirow{2}{*}{$\begin{array}{c}\text { PWR } \\
\text { (Sizewell-B) }\end{array}$} & $\begin{array}{l}\text { Grid } \\
\text { frequency } \\
\text { error } \\
\text { injection test }\end{array}$ \\
\hline & & & & $\begin{array}{l}\text { Single } \\
\text { turbine } \\
\text { trip event }\end{array}$ \\
\hline 13 & $\begin{array}{l}\text { TACIS R2.30/94 Project Transient Analysis for } \\
\text { RBMK Reactors } \\
\text { H. Schoels, Yu. M. Nikitin Nikiet }\end{array}$ & $\begin{array}{l}\text { FLICA GIDRA SADC } \\
\text { DINAO } \\
\text { CRONOS } \\
\text { QUABOX/CUBOX }\end{array}$ & $\begin{array}{c}\text { RBMK } \\
\text { (Smolensk 3) }\end{array}$ & RIA \\
\hline 14 & $\begin{array}{l}\text { PWR Anticipated Transients Without } \\
\text { SCRAM Analyses Using PVM Coupled } \\
\text { RETRAN and STAR 3-D Kinetics Codes } \\
\text { M. Feltus, K. Labowski }\end{array}$ & $\begin{array}{l}\text { RETRAN } \\
\text { STAR 3-D }\end{array}$ & PWR & ATWS \\
\hline 15 & $\begin{array}{l}\text { Development and First Results of } \\
\text { Coupled Neutronic and Thermal-hydraulics } \\
\text { Calculations for the } \\
\text { High-performance LWR } \\
\text { C.H.M. Broeders, V. Sanchez-Espinoza, A. } \\
\text { Travleev }\end{array}$ & $\begin{array}{l}\text { RELAP5 } \\
\text { KAPROS }\end{array}$ & HPLWR & FA tests \\
\hline 16 & $\begin{array}{l}\text { Analysis and Calculation of an } \\
\text { Accident with Delayed Scram } \\
\text { on NPP Greifswald using the } \\
\text { Coupled Code DYN3D-ATHLET } \\
\text { S. Kliem }\end{array}$ & $\begin{array}{l}\text { ATHLET } \\
\text { DYN3D }\end{array}$ & $\begin{array}{l}\text { VVER-440 } \\
\text { (Greifswald) }\end{array}$ & $\begin{array}{l}\text { Delayed } \\
\text { scram }\end{array}$ \\
\hline 17 & $\begin{array}{l}\text { Multi-dimensional TMI-1 Main Steam Line } \\
\text { Break Analysis Methodology using TRAC- } \\
\text { PF/NEM } \\
\text { K. Ivanov, T. Beam, A. Baratta, A. Irani, N. } \\
\text { Trikouros }\end{array}$ & $\begin{array}{l}\text { TRAC-PF } \\
\text { NEM }\end{array}$ & $\begin{array}{c}\text { PWR } \\
(\text { B\&W TMI-1) }\end{array}$ & MSLB \\
\hline 18 & $\begin{array}{l}\text { Realistic and Conservative Rod Ejection } \\
\text { Simulation in a PWR Core at HZP, EOC } \\
\text { with Coupled PARCS and RELAP Codes } \\
\text { J. Riverola, T. Núñez, J. Vicente }\end{array}$ & $\begin{array}{l}\text { RELAP } \\
\text { PARCS }\end{array}$ & $\begin{array}{c}\text { Three-loop } \\
\text { PWR }\end{array}$ & $\begin{array}{l}\text { Peripheral } \\
\text { rod ejection }\end{array}$ \\
\hline 19 & $\begin{array}{l}\text { OECD/NRC BWR } \\
\text { Benchmark } 3^{\text {rd }} \text { Workshop }\end{array}$ & $\begin{array}{c}\text { ATHLET } \\
\text { QUABOX/CUBBOX }\end{array}$ & $\begin{array}{l}\text { BWR Peach } \\
\text { Bottom }\end{array}$ & TT \\
\hline
\end{tabular}

Table 1.(continues) Overview of 3-D coupled neutronics/thermal-hydraulics calculations available from the literature 


\subsection{Computational Fluid Dynamics (CFD) codes}

The strategy of CFD is to replace the continuous domain with a discrete domain using a grid. The geometry is discretized with a typical mesh size of less than a volume and the thermalhydraulics properties are computed for every grid point defined. The conservation equations for mass momentum and energy are solved in a discrete form. Any complex geometry is possible, the extremely fine resolution costs computation time. The CFD approach is mostly preferred for small geometries. Existing CFD codes include: FLUENT, CFX.

\section{Coupled neutronic and thermal-hydraulics computer codes for LWR}

An overview of available coupled neutronics/thermal-hydraulics code published up to now has been reported in table 1 . This table summarizes a list of coupled codes for PWR, BWR to date, with the computer codes described in the previous chapters.

\subsection{Requirements to the coupling algorithm}

Detailed description of the interlace requirement to couple thermal-hydraulics code to 3-D neutronic code has been reported by Langenbuch et al. The objective to couple neutronics code with a thermal-hydraulics code is to provide an accurate solution in a reasonable amount of CPU time. For the present study, the basic components that are considered for the coupling methodology include:

\subsection{Coupling method}

There are two different ways of coupling, internal and external coupling. With internal coupling the neutronics code is integrated within the thermal-hydraulics code. While with external coupling, the two codes run externally and exchange information between each other.

\subsection{Spatial mesh overlay}

Accurate mapping of mesh or volumes between the two codes is important to exchange information between each other.

\subsection{Coupled convergence schemes}

A convergence scheme of the two codes needs to be defined. For a final convergence of the coupled codes, independent convergence in the individual codes is required.

\section{Theory of Artificial Neural Network (ANN)}

An ANN consists of simple computational units called neurons and it is characterized by a network structure. The neurons connected to each other with different connection strengths. The strength of a connection between neurons is called weight. The types of ANNs are different and associated with applications. The artificial neural networks have a wide variety of applications in nuclear engineering. Some of the basic related researches are listed below:

- Fuel management optimization (Faria and Pereira, 2003)

- Prediction of core parameters (Gazula and Bohr, 1992)

- Plant control and monitoring (Uhrig, 1995) 
- Nonlinear dynamics and transient diagnosing (Adali et al., 1997)

- Two-phase flow study (Tambouratzis and Pazsit, 2009)

- $\quad$ Signal validation method (Ikonomopoulos and Van Der Hagen, 1997)

In some investigations to speed up effectively optimization process a very fast estimation system of core parameters has been introduced and developed using cascade feed forward type of artificial neural networks.

\subsection{ANN designing}

Among the literature, there are different types of available network architectures. The most popular neural network is Multi-Layer Perceptron (MLP) network. This later has been chosen because of its high performance in predictive tasks (Erdogan and Geckinli, 2003; Souza and Moreira, 2006) and to let comparison with the results issued from our calculations. In MLP, various neurons are arranged in different layers called input, hidden, and output. Fig. 1 shows a typical scheme of the three layers neural network. The neurons in the first layer correspond to independent input variables of the problem and transmit the input values to the succeeding layer. After the input layer, there may be one or more hidden layers. They receive the weighted combination of input values from the preceding layer and produce an output depending on their activation function (Jodouin, 1994). As shown in figure 1, the weights are determined and adjusted, through an iterative and a backpropagation process, minimizing a quadratic error function. Thus, to make use of an appropriate Artificial Neural Network, one must fine-tune the following items as their incidence on the prediction parameters are of a crucial importance.

The items of interest are as follow:

1. Activation function,

2. Performance function,

3. Training algorithms.
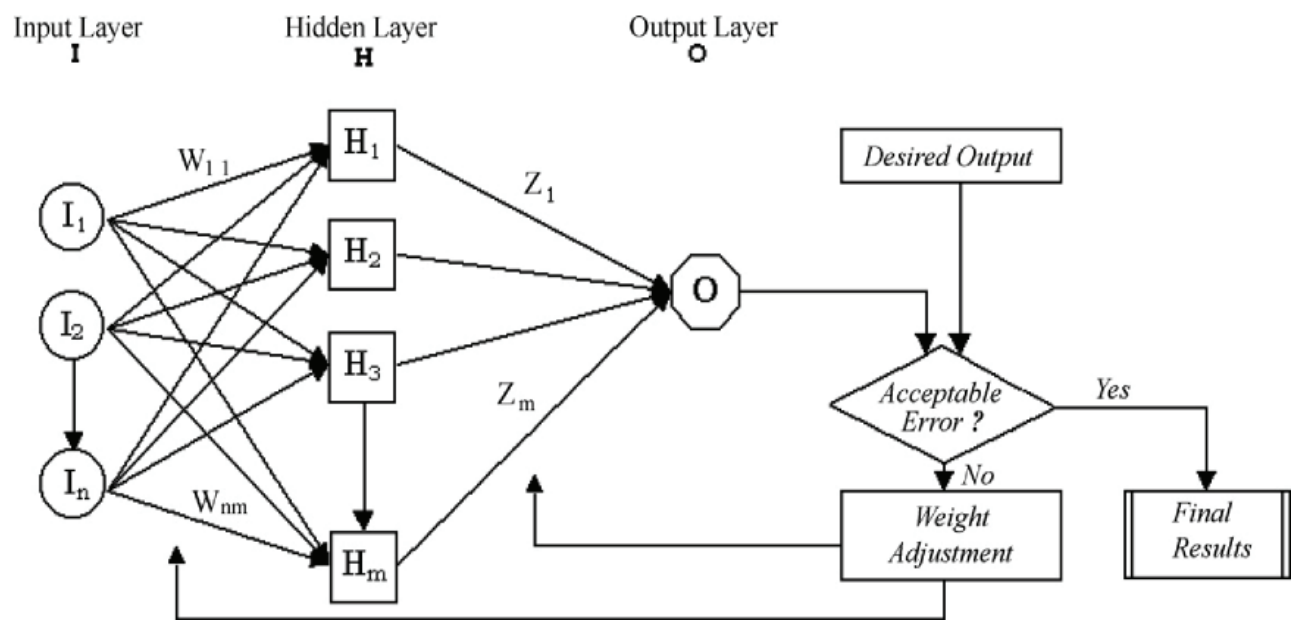

Fig. 1. Typical architecture of Multi-Layer Perceptron (MLP) neural network 


\subsection{Cascade feed forward neural networks}

A general type of feed-forward ANNs consists of a layer of inputs, a layer of output neurons, and one or more hidden layers of neurons. Figure 2 shows a general type of a three layers feed-forward ANN. Typically feed-forward ANNs are used to parameter prediction and data approximation.

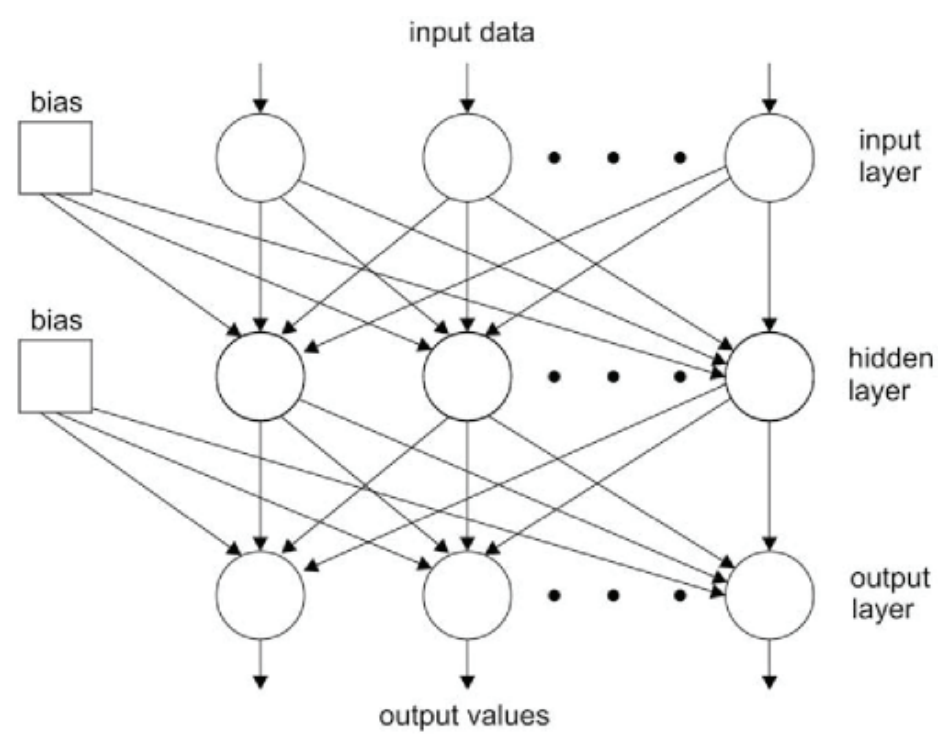

Fig. 2. A general type of three layered feed-forward ANNs

A cascade type of feed-forward ANNs consists of a layer of input, a layer of output neurons, and one or more hidden layers. Similar to a general type of feed-forward ANNs, the first layer has weights coming from the input. But each subsequent layer has weights coming from the input and all previous layers. All layers have biases. The last layer is the network output. Each layer's weights and biases must be initialized. A supervised training method is used to train considered cascade feed forward ANNs.

\subsection{Training and activation functions}

The training process determined through a back propagation algorithm which minimizes a quadratic error between the desired and network outputs. The gradient descent method with momentum weight/bias learning rule has been used to train considered ANNs. It is a developed algorithm of the basic back propagation algorithm (Hagan et al., 1995; Rumelhart et al., 1986a,b). A net input $\left(\mathrm{V}_{\mathrm{j}}\right)$ to a neuron in a hidden layer $\mathrm{k}$ is calculated by this formula (Eq. (1)).

$$
V_{j}=\sum_{i=1}^{n} W_{j i} \theta_{i}+\theta_{j}
$$

Where $\mathrm{n}$ is the number of $\mathrm{k}-1$ layer neurons for a general type of feed-forward ANNs and the number of all of the previous layer neurons for a cascade type of feed-forward ANNs. Weights are noted by $W_{\mathrm{ji}}$; and the threshold offset by $\theta_{\mathrm{j}}$. 
The output of the neuron $\mathrm{O}_{j}$ is given by an activation function. An activation derivative function effects on neuron outputs to compress propagated signals and simulate the nonlinearity of the complex systems. Many different activation functions are used in feedforward ANNs. There are several types of activation functions such as Linear (Eq. (2)), LogSigmoid (Eq. 3), Tan-Sigmoid (Eq. 4) functions, etc.

$$
\begin{gathered}
O_{j}=\operatorname{Pureline}\left(V_{j}\right) \\
O_{j}=\operatorname{Logsig}\left(V_{j}\right)=1 /\left(1+e^{-\left(V_{j}\right)}\right) \\
O_{j}=\operatorname{Tansig}\left(V_{j}\right)=\left(1-e^{-2\left(V_{j}\right)} /\left(1+e^{-2\left(V_{j}\right)}\right)\right.
\end{gathered}
$$

In this learning method, which is a batch training method, weights and biases are only updated after all the inputs and targets are presented to ANNs. Then the average of system error (Eq. 5) should be minimized to increase learning performance.

$$
E_{A V}=\frac{1}{2 N} \sum_{i=1}^{N} \sum_{j=1}^{M}\left(d_{j}(n)-O_{j}(n)\right)^{2}
$$

Where $d_{j}(n)$ is the desired output; and $\mathrm{O}_{j}(n)$ is the network output. $\mathrm{N}$ and $\mathrm{M}$ are the total number of training data sets and the number of neurons of the output layer. In the gradient descent method improved values of the weights can be achieved by making incremental changes $\Delta \mathrm{W}_{\mathrm{ji}}$ proportional to $\partial \mathrm{E}_{\mathrm{AV}} / \partial \mathrm{W}_{\mathrm{ji}}$ (Eq. 6).

$$
\Delta W_{j i}=-\eta \frac{\partial E_{A V}}{\partial W_{j i}}
$$

Where the proportionally factor $\eta$ is called the learning rate. Large values of $\eta$ in the gradient descent formulation may lead to large oscillation or divergence. One attempt to increase the speed of convergence while minimizing the possibility of oscillation, or divergence, involves adding a momentum term to the basic gradient descent formulation. In this case the weight vector at time index $(\mathrm{k}+1)$ is related to the weight vectors at time indexes (k) and (k-1) by this formula (Eq. 7).

$$
W(k+1)=W(k)-\left[\eta \frac{\partial E}{\partial W}+\beta \Delta W(k-1)\right]
$$

Then the new weights for step $(\mathrm{k}+1)$ are given by:

$$
\Delta W_{j i}(k+1)=\eta \delta_{j} O_{j}+\beta \Delta W_{j i}(k)
$$

Where a momentum coefficient, or an acceleration parameter $\beta$ is used to improve convergence. The expression of $\delta_{j}$ is given by:

$$
\begin{gathered}
\delta_{k}=0.5\left(d_{k}-O_{k}\right) f^{\prime}\left(v_{k}\right) \\
\delta_{j}=f^{\prime}\left(v_{k}\right) \sum_{k} \delta_{k} W_{k j} \text { for hidden neurons }
\end{gathered}
$$


It should be noted that the technology of ANNs has been still developing. The determination of minimum number of necessary hidden neurons and hidden layers is completely practical. If the hidden neurons are chosen very small, the network will classify its input in a small number of classes (Wilde, 1997). If the hidden neurons are selected extremely large, the time of learning process increases ineffectively. Presently, the best method is making an educated guess. In this work, after primarily studies some practical tests are suggested and used to adjust the main parameters and properties of the ANNs' structures and used training rule (Eqs. 1 through 10).

\subsection{ANN development strategy}

The motivation in using such a computational procedure lies in the fact that it will let us use just hundreds of configurations rather than the thousands, in the learning stage, that are usually required in typical calculations to ensure reasonable predictions. Hence, as shown in Fig. 3, a suitable neural networks development strategy can be tested based on executing the following two main calculational stages, in an independent way: learning stage and prediction stage.

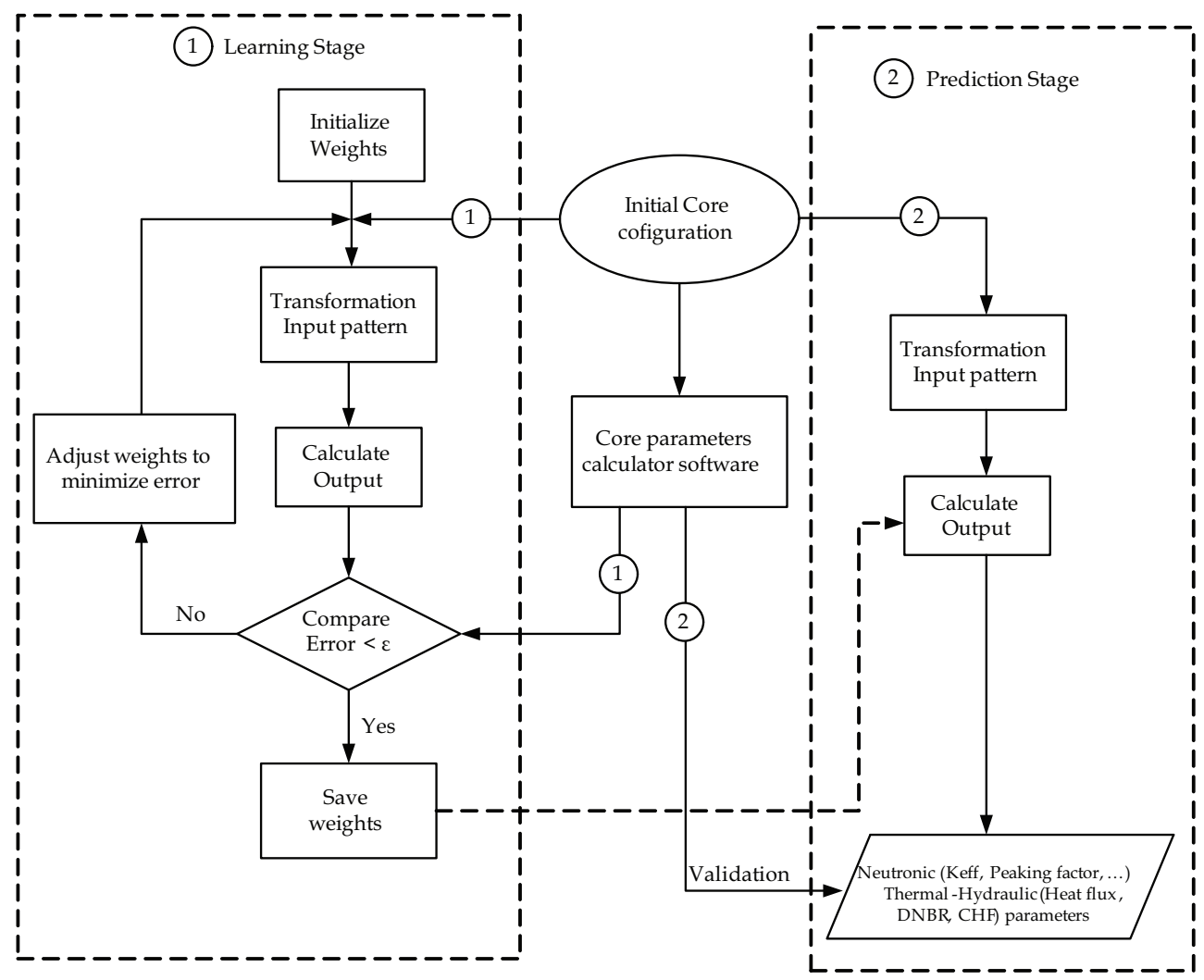

Fig. 3. Overall back-propagation computational strategy for the core parameter prediction 
The first stage of computational procedure consists of creating suitable networks by applying an appropriate learning rule using a desired database. The information required in the related database will contain coupled input values with the corresponding target output values. These values are used to train the networks until the error reaches a desired value stated at the beginning of the learning process. It becomes evident that the quality of the results obtained will depend on how well knowledge is capitalized in this database. Hence, significant attention will be focused on how well this database will be created. The main steps required in the learning process are:

1. Create the database for training;

2. Construction of networks for training;

3. Choosing a learning function;

4. Train the developed networks;

The second stage is the prediction one where the weights, from the inter-connected neurons, have been adjusted to the desired error in the previous calculations stage. These weights will be used in a global computational sequence, to predict the networks outputs when unseen data will be presented to the developed networks. This is the power of the network approach and one of the reasons for using it. The net is said to have been generalized from the training data. This stage is necessary to test the performance of the developed neural network.

\subsection{Create data-base for training}

A wide variety of completely different core arrangements are needed to train effectively considered ANNs. In this work, the fuel assembly positions are considered changeable in calculations. Core calculations have been done by a supporting software tool that will be able to calculate neutronic and thermal hydraulic parameters of a typical reactor core. This program uses a coupling method to calculate reactor core parameters for desired core configuration. Needed parameters for training should be extracted from the software calculations. They must be converted to a compatible format to feed desired ANNs. Doing this manually takes a long time while some human errors are possible. In this research, a data base builder program is designed and used. It is used to create data sets necessary to train and test considered ANNs.

In this research, a software package (Core Parameters Calculator) is developed and used. The random state of the software is used to create data sets necessary to train and test used ANNs. Many strings composed of specific integer numbers are chosen randomly to form different core configurations. For each different state (configuration), Core Parameters Calculator software uses MCNP and COBRA-EN code to extract needed neutronic and thermal-hydraulics core parameters. During calculation process, MCNP code uses cross sections library provided by NJOY program. Then calculated fission powers of fuel rods send to Thermal-hydraulics code for calculating of density and temperature distribution of fuel and coolant. Finally the results (consist of neutronic and thermal- hydraulic parameters) are stored on a local data base table. Figure 4 shows the main diagram of creating desired data.

\subsection{Developing of a supporting tool for core parameters calculation}

Due of the strong link between the water (moderation) and the neutron spectrum and subsequently the power distribution, a coupling of neutronics and thermal-hydraulics has 


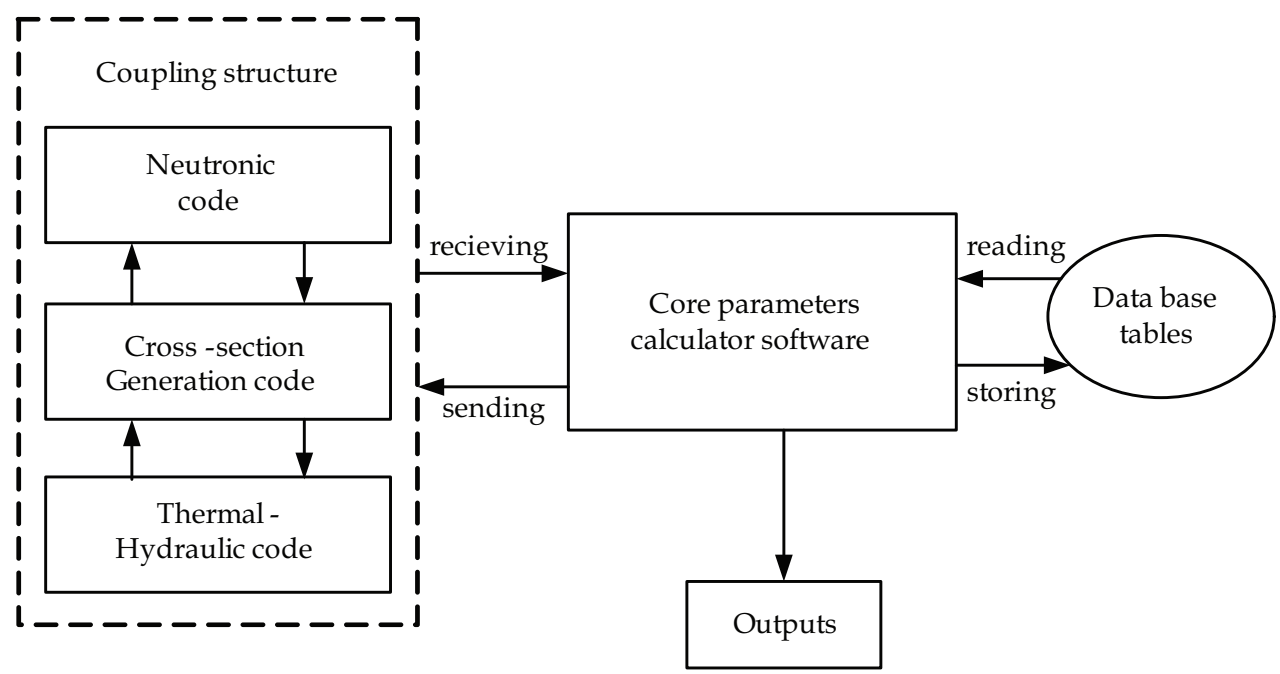

Fig. 4. The main diagram of creating desired data

become a necessity for reactor concepts operating at real conditions. The effect of neutron moderation on the local parameters of thermal-hydraulics and vice-verse in a fuel assembly has to be considered for an accurate design analysis. In this study, the Monte Carlo NParticle code (MCNP) and the sub-channel code COBRA-EN (Sub-channel Thermalhydraulics Analysis of a Fuel Assembly for LWR) have been coupled for the design analysis of a fuel assembly and core with water as coolant and moderator. Both codes are well known for complex geometry modeling. The MCNP code is used for neutronics analyses and for the prediction of power profiles of individual fuel rods. The sub-channel code COBRA for the thermal- hydraulics analyses takes into account the coolant properties as well as separate moderator channels.

The coupling procedure is realized automatically. MCNP calculates the power distribution in each fuel rod, which is then transferred into COBRA to obtain the corresponding thermalhydraulics conditions in each sub-channel. The new thermal-hydraulics conditions are used to generate a new input for the next MCNP calculation. This procedure is repeated until a converged state is achieved. The parameters that are exchanged between the two codes for the coupling are: power distribution from MCNP code, water density distribution, water temperature distribution and fuel temperature distribution from COBRA code, as shown in Figure 5. The COBRA-EN code, which is written in FORTRAN language, is modified to include the power distribution obtained from neutronics analysis and to be able modeling of Russian fuel type.

The nuclear cross section data library of MCNP must be provided for additional temperatures and must be added to MCNP data directory. The cross section data for neutron interaction are obtained from the evaluated MCNP libraries ENDF/B. Cross section data provided with the MCNP are for a limited number of temperatures. An additional library must be constructed from NJOY code with more temperatures $(300 \mathrm{~K}, 500 \mathrm{~K}, 600 \mathrm{~K}$, $760 \mathrm{~K}, 800 \mathrm{~K}, 1000 \mathrm{~K}, 1500 \mathrm{~K})$ and is added to the MCNP data directory. The coupled code system was tested on a proposed fuel assembly design of a VVER-1000. The coupling 
procedure presented will also be applicable to other types of reactors with a density variation in the core such as in BWR.

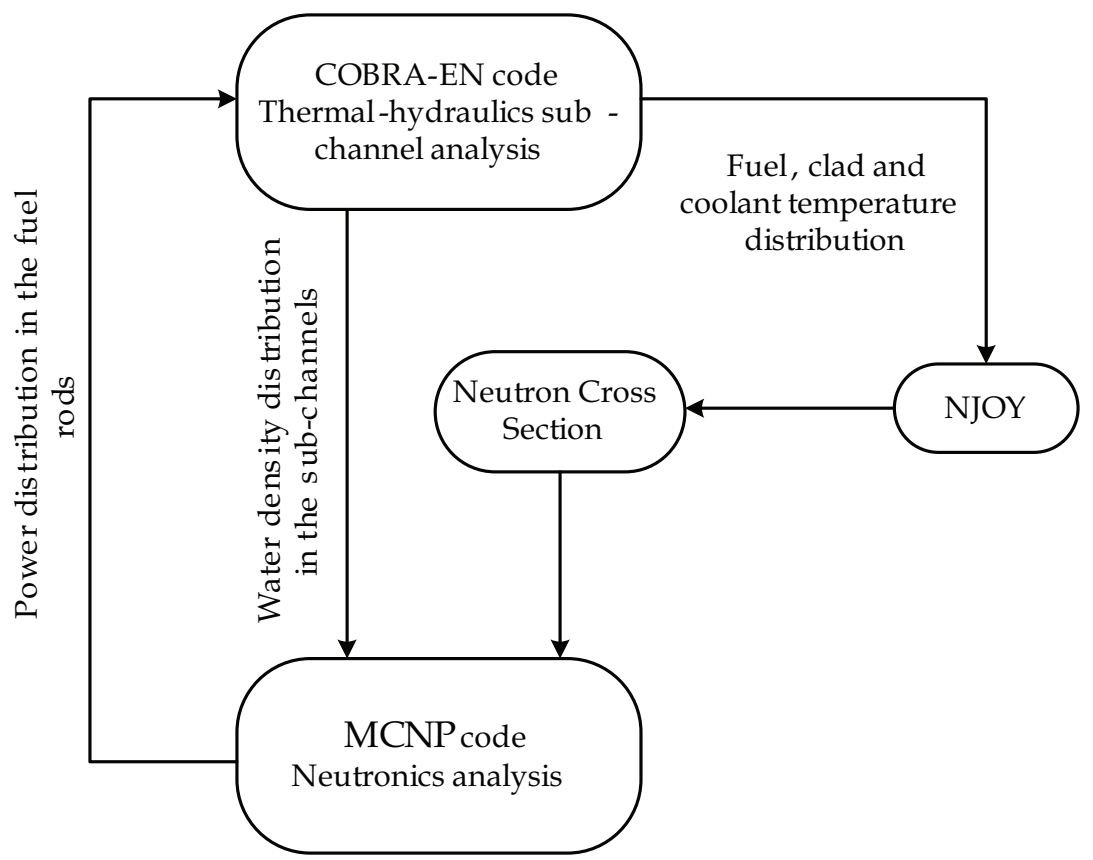

Fig. 5. Coupled MCNP/COBRA-EN for joining neutronic -thermalhydraulics are shown schematically. The cross sections modification are a major concern which are doen using NJOY code

From the literature review, most of the available coupled codes for neutronics/thermalhydraulics are based on diffusion and system codes resulting in a rather coarse resolution of the core. For a detailed analysis of a VVER-1000 fuel assembly analysis, diffusion codes and system codes are not giving enough local information. All prior application had been to PWR and BWR transient analysis. To accurately analyze a VVER fuel assembly a more detailed analysis fuel rod wise and sub-channel wise is required to predict a hot spot and the temperature distribution around the circumference of a fuel rod. In order to perform such detailed analysis of the VVER fuel assembly, a new coupled code system is required. From the reviewed neutronics and thermal-hydraulics computer codes, the Monte Carlo code and sub-channel codes show to be the best choice of codes to be coupled for detailed fuel assembly analysis. Both have similar spatial resolution. The smallest control volume is in the order of a few $\mathrm{cm}$ in both cases. System codes on the other hand would be too coarse for MCNP and CFD codes too fine in resolution.

\section{Conclusions}

Obviously, due to huge files, it is not possible to present our input files ( MCNP and COBRA-EN codes) as our suggested package in this chapter, but reader can consult the 
corresponding author to find the MCNP as well as COBRA-EN input files for simulating a VVER-1000 reactors. The MCNP code contains hexagonal core including all core conditions such as all control rod inserted (or withdrawn), boric acid inserted, hot full power condition, etc. Also, reader can find our COBRA-EN code to undrestand how we can simulate thermal hydraulics subchannels of a VVER-1000 reactor. Moreover, as we said previously, temperature cross sections modification are carried out using NJOY code and obviously reader can receive our modification. These so-called data are used as output data for ANN training. If reader are interested, they can consult the corresponding author to get our ANN simulator. Basically, the main objective of the ANN software is to obtain fast estimation tool which allows large explorations of core safety parameters. This software is very useful in reactor core designing and in-core fuel management or loading pattern optimization.

In due course, verification and validation of the procedures are taking into account using available experimental data or other code-to-code benchmarking, and this is an important part of research.

\section{References}

Adali, T., Bakal, B., Sönmez, M.K., Fakory, R., Tsaoi, C.O., 1997. Modeling nuclear reactor core dynamics with recurrent neural networks. Neurocomputing 15 (3-4), 363381.

Allaire, G.: Solving Linear System Equation in FLICA, A Thermo-Hydraulic Code for 3-D Transient Computations, Proc. International Conference on Mathematic and Computations, Reactor Physics and Environmental Analyses.

Asaka, H., Zimin, V.G., Iguchi, T., Anoda, Y.: Coupling of the Thermal-hydraulics codes with 3D Neutron Kinetic Code SKETCH-N, Preliminary Proceedings of the OCED/CSNI Workshop on Advanced Thermal-hydraulics and Neutronics Codes: Current and Future Applications, Vol.2, pp. 1 - 15, Barcelona, Spain, 2000

Bousbia-Salah, A. et al.: Analysis of the Peach Bottom Turbine Trip 2 Experiment by Coupled RELAP-PARCS Three-Dimensional Codes, Nuclear Science and Engineering, Vol. 148, pp337- 353, 2004.

Bovalini, R., D'Auria, F., Galassi, G.M., Spadoni, A., Hassan, Y.: TMI-MSLB Coupled 3-D Neutronics/Thermal-hydraulics Analysis: Application of RELAP5-3D and Comparison with Different Codes, RELAP5 International Users Seminar, Sun Vally, Idaho, 2001.

Briesmeister J.F, Editor, MCNP - A General Monte Carlo N-Transport code, Version 4C, Los Alamos National Laboratory report LA-12625, 1993.

Broeders, C.H.M., Dagan, R., Sanchez-Espinoza, V, Travleev, A.: KAPROS-E: Modular Program System for Nuclear Reactor Analysis, Status and Results of Selected Applications, Jahrrestagung Kerntechnik, Diisseldorf, 2004.

Burwell, M.J.,Lerchl, G., Miro, J., Teschendorff, V., Wolfert, K.: The Thermal-hydraulics Code ATHLET for Analysis of PWR and BWR Systems, Proceedings Fourth International Topical Meeting on Nuclear Reactor Thermal-hydraulics, Vol. 2, pp 1234 - 1239, Oct. 10 - 13th,1989.

CFX-4 User Manual,1997, AEA Technology, http://www.software.aeat.com/cfx.default.asp 
Cheng, X., Schulenberg, T., Bittermann, D., Rau, P.: Design Analysis of Core Assemblies for Supercritical Pressure Condition, Nuclear Engineering and Design, 223, 279-294, 2003.

Erdogan, A., Geckinli, M., 2003. A PWR reload optimisation code (XCore) using artificial neural networks and genetic algorithms. Ann. Nucl. Energy 30,35-53.

Faghihi, F., Fadaie, A.H., Sayareh, R. Reactivity coefficient simulation of the Iranian VVER1000 nuclear reactor using WIMS and CITATION codes. Prog. Nucl. Energy 49, 6878. 2007.

Faghihi, F.; Saidinezhad, M.; 2011. Two safety coefficients for $13 \times 13$ annular fuel assembly, Prog. Nuclear Energy 53, pp.250-254.

Faghihi et al.; 2010. Modified COBRA_EN code for investigating Iranian VVER-1000 reactor, Prog. Nucl. Energy 52, pp. 289-295.

Faria, E.F., Pereira, C., 2003. Nuclear fuel loading pattern optimization using a neural network. Annals of Nuclear Energy 30 (5), 603-613.

Fluent 5 Users Guide, Fluent Inc., Lebanon, NH (1998); http:/ / www.fluent.com.

Fu, H., Rodarte, J.S., Ivanov, K.N.: TRAC-PF1/NEM Modelling and Results of OECD/NEA BWR core Transient Benchmarks, Annals of Nuclear Energy, 27, $1051-1058,2000$.

Gazula, S., Bohr, J.W.C., 1992. Learning and prediction of nuclear stability by neural networks. Nuclear Physics A 540 (1-2), 1-26.

Glaeser, H.: Validation and Uncertainty Analysis of the ATHLET code Thermal-hydraulics computer code, Nuclear Society of Slovenia, 2nd Regional Meeting: Nuclear Energy in Central Europe Portoroz, Slovenia, 1995.

Grundmann, U., S. Mittag and U. Rohde, Dyn3d2000/M1 for the Calculation of Reactivity Initiated Transients in LWR with Hexagonal and Quadratic Fuel Elements - Code Manual and Input Data Description for Release, 3rd Edition, Research Center Rossendorf Inc., Sept. 2001.

Grundmann, U., Lucas, D., Rohde, U.: Coupling of the Thermo-hydraulic Code ATHLET with the Neutron Kinetic Core Model DYN3D, Proc. International Conf. on Mathematics and Computation, Reactor Physics and Environmental Analyses.

Grundmann, U., Kliem, S., and Rohde, U.: Analysis of the Boiling Water Reactor Turbine Trip Benchmark with the Codes DYN3D and ATHLET/DYN3D. Nuclear Science and Engineering, Vol. 148, Page 226 - 234, 2004.

Hagan, M.T., Demuth, H.B., Beale, M.H., 1995. Neural Network Design. PWS Pub. Co., Har/Dsk Edition.

Holland, J.H., 1975. Adaptation in Natural and Artificial Systems. University of Michigan, Ann Arbor.

Ikonomopoulos, A., Van Der Hagen, T.H.J.J., 1997. A novel signal validation method applied to a stochastic process. Annals of Nuclear Energy 24 (13), 1057-1067.

Ivanov, K., et al., "Nodal Kinetic Model Upgrade in The Penn State Coupled TRAC/NEM Codes", Annl. Nucl. Ener., 26, 1205 (1999).

Ivanov K.N., Juan, R.M., Irani, A., Baratta, A.J.: Features and Performance of a Coupled Three Dimensional Thermal-hydraulics/kinetics TRAC-PF1/NEM PWR analysis code, annals of Nuclear Energy 26, 1407 -1417, 1999. 
Jackson, C.J., Finnemann, H.: Verification of the Coupled RELAP/PANBOX System with the NEACRP LWR Core Transient Benchmark, Proc. International Conference on Mathematic and Computations, Reactor Physics and Environmental Analyses

Jodouin, J.F., 1994. Les Réseaux Neuromimétiques, Modèles et Applications. Edit. Hermès, Paris.

Joo, H.G., D.A. Barber, G. Jiang and T.J. Downar, PARCS: A Multidimensional Two-group Reactor Kinetic Code Based on the Non-linear Analytical Nodal Method, University of Purdue Report PU/NE-98-26 (1998).

Kim, H.G., Change, S.H., Lee, B.H., 1993. Pressurized water reactor core parameter prediction using an artificial neural network. Nuclear Science and Engineering $113,10-76$.

Kirkpatrick, S., Gellat, C.D., Vecchi, M.P., 1983. Optimization by simulated annealing. Science 220 (4598), 671-680.

Langenbuch, S., QUABBOX/CUBBOX-HYCA, Ein Dreidimensionales Kernmodell mit parallelen Kühlkanälen für Leichtwasser-reaktoren, GRS-A-926, Garching, Germany (1984).

Langenbuch, S., Austregesilo, P., Fomitchenko, P., Rohde, U., Velkov, K.: Interface Requirements to Couple Thermal-Hydraulics Codes to 3D Neutronic Code, OCED/CSNI Workshop on Transient Thermal-hydraulics and Neutronic Codes Requirements, Annapolis, United State, 1996.

Lee, D et al.: Analysis of the OECD/NRC BWR Turbine Trip Transient Benchmark with the coupled Thermal-hydraulics and Neutronics Code TRAC-M/PARCS, Nuclear Science and Engineering, Vol. 148, Page 291 - 305, 2004

Lee, D., Downar, T.J., and Kim, Y.: A Nodal and Finite Difference Hybrid Method for Pin-by Pin Heterogeneous Three-Dimensional Light Water reactor Diffusion Calculations, Nuclear Science and Engineering, Vol. 146, pp. 319 - 339, 2004

Mazrou, H., Hamadouche, M., 2004. Application of artificial neural network for safety core parameters prediction in LWRRS. Progress in Nuclear Energy 44 (3), 263-275. Fuel and Energy Abstracts 46 (1), 14, January 2005.

Miller, M.R., Downar, T.J.: Completion Report for the Coupled TRACS-M/PARCS Code, University of Purdue, Report PU/NE-99-20.

Mignot, G., Royer, E., Rameau, B., Todorova, N.: Computation of a BWR Turbine Trip with CATHARE-CRONOS2-FLICA4 Coupled Codes, Nuclear Science and Engineering, Volume 148, Page 235 - 246, 2004.

Misu, St., Kiehlmann, H.D., Spierling, H., Wehle, F.: The Comprehensive Methodology for Challenging BWR Fuel Assembly and core Design used in Framatome ANP, Physor, Seoul, Korea, 2002.

Mori, M., Rineiski, A., Kretzschmar, F., Maschek, W., Morita, K.: Coupled MCNP/MXN Calculations for the SCFR, CAPRA-CADRA, International Seminar, Aix-enProvence, France,2004.

Mori, M., Maschek, W., Laurien, E., Morita, M.: Monte-Carlo/Simmer-III Reactivity Coefficient Calculation for the Super-Critical Water Fast Reactor, Proc. of the ANS/ENS Topical Meeting GLOBAL, New Orleans, 2003. 
Nigro, A.L., Spadoni, A., D'Auria, F., Saiu, G.: MSLB Coupled 3D Neutronics-ThermalHydraulics Analysis of a Large PWR using RELAP5-3D, International Conference Nuclear Energy in Central Europe, Portoroz, Slovenia, 2001.

Oak Ridge National Laboratory, 1972. CITATION-LDI2 code.

Pautz, A., and Birkhofer, A.: DORT-TD: A Transient Neutron Transport Code with Fully Implicit Time Integration, Nuclear Science and Engineering, Vol. 145, pp. 299 319, 2003

Pautz, A., Hesse, U., Zwermann, W., Langenbuch, S.: Fuel Assembly Calculation Using the Method of Discrete Ordinates, Nuclear Science and Engineering, Vol. 149, pp. 197 $-210,2005$.

Pazsit, I., Kitamura, M., 1996. The rule of neural networks in reactor diagnostics and control. Advances in Nuclear Science and Technology 24, 95-130.

Rhoades W. A., Childs R. L.: TORT-DORT, Two- and Three-Dimensional Discrete Ordinates Transport, Version 2.7.3, RSIC-CCC-543, ORNL RSICC, Oak Ridge, TN (1993).

Rumelhart, D.E., Hinton, G.E., Williams, R.J., 1986b. Learning internal representations by error propagation. In: Parallel Data Processing, vol. 1. The MIT Press, Cambridge, MA, pp. 318-362. (Chapter 8).

Sanchez-Espinoza, V.H., Hering, W., Knoll, A., Boeer, R.: Analysis of the OCED.NEA PWR Main Steam Line Break (MSLB) Benchmark Exercise 3 with coupled code system RELAP5/PANBOX, Wissenschaftliche Berichte, FZKA- 6518, 2002.

Sanchez-Espinoza, V., Hering, W., Knoll, A.: Analysis of the OECD/NEA PWR MSLB Benchmark Exercise 1 using the RELAP5 Code with Point Kinetics Option, FZKA 6427, 2002.

Sanchez-Espinoza, V., Hering, W.: Investigations of the Appropriateness of RELAP5/MOD3 for the Safety Evaluation of an Innovative Reactor Operated at Thermodynamically Supercritical Conditions, FZKA 6749, 2002.

Souza, R.M.G.P., Moreira, J.M.L., 2006. Neural network correlation for power peak factor estimation. Ann. Nucl. Energy 33, 594-608.

Turinsky, P.J., et al., NESTLE: A Few-group Neutron Diffusion Equation Solver Utilizing the Nodal Expansion Method for Eigenvalue, Adjoint, Fixed-source Steady State and Transient Problems, EGG-NRE-11406, Idaho National Engineering Laboratory, June 1994.

Uhrig, R.E., 1991. Potential application of neural networks to operation of nuclear power plants. Nuclear Safety 32.

Uhrig, R.E., 1993. Use of neural networks in nuclear power plants. ISA Transactions 32 (2), 139-145.

Wheeler, C.L., Stewart, C.W., Cena, R.J., Rowe, D.S., Sutey, A.M.: COBRA-IV -I, An Interim Version of COBRA for Bundle Nuclear Fuel Element and Cores, BNWL-1962, UC32, March 1976.

Wilde, P.D., 1997. Neural Network Models. Theory and Projects. Springer, London, p.40.

Winfrith, 1982. LWR-WIMS, a Computer Code for Light Water Reactor Calculations. AEE, UK. AEEW-R 1498.

Yoo, Y.J., and Hwang, D.H.: MATRA, Multichannel Analyzer for Steady States and Transients in Rod Arrays, Korea Atomic Energy Research Institute, October 2003. 
Ziabletsev, D.N., Ivanov, K.N.: Improved verification methodology for TRAC-PF1/NEM Using NEA/OECD Core Transient Benchmarks, Annals of Nuclear Energy, 27, 1319 - 1331, 2000. 


\title{
Recent Trends in Mathematical Modeling and Simulation of Fission Product Transport From Fuel to Primary Coolant of PWRs
}

\author{
Nasir M. Mirza, Sikander M. Mirza and Muhammad J. Iqbal \\ Department of Physics and Applied Mathematics, \\ Pakistan Institute of Engineering and Applied Sciences, Nilore, Islamabad 45650,
}

Pakistan

\section{Introduction}

With over 437 operational power plants, nuclear systems contribute $370705 \mathrm{MW}(\mathrm{e})$ worldwide [1]. The Pressurized Water Reactors (PWR) constitute a two-third majority of the operational nuclear power plants while the nuclear reactors in planning and construction phases also show strong trend towards PWRs. These systems are mainly used as baseline load carriers while conventional fossil fueled systems are used for load adjustments and variations [2]. The PWRs have higher than average levels of radiation fields emanating from the corrosion and fission product activity [3] [4] [5]. This leads to prolongation of maintenance schedules entailing loss of revenues mounting to several million dollars per plant annually [6]. Consequently, the plant availability factors are also lowered. This situation is further aggravated due to strong shift of plant age profile toward over 25 years operational range. With plant aging, the fuel failures become more frequent which leads to enhancement of radiation levels in the primary circuits of PWRs.

The levels of fission product activity (FPA) have been of concern both from the operational as well as from accidental perspectives. These levels are continuously monitored during the normal operation of PWRs. The fuel pins develop leakages with their burnup. When the failed fuel fraction exceeds a safe limit, replacement of defective assemblies by refueling becomes necessary. Therefore, low levels of leaked-out fission products (FPs) in primary coolant of PWRs are indicative of the core health [7]. In the accidental conditions, the total value of FPA serves as the available source term that potentially can escape into the surroundings [8] [9].

The fission products are released in the fuel matrix during burnup. They escape from the ceramic pellets into the gap between pellets and the clad regions. Hyun et al. [10] have developed an analytic method for the fuel rod gap inventory of unstable fission products during steady state operation of PWRs. The fission gas bubbles escape from grain corners and are interlinked in the open space. The release rate depends on the bubble interlinkage along with temperature and burnup. A generalized model for fission product transport in the fuel-to-sheath gap was given by Lewis [11]. Barrachin et al. presented a review of fission product behaviour in $\mathrm{UO}_{2}$ fuel [12]. 
The release of fission products from fuel-clad gap into primary coolant involves clad failure. A model describing pallet oxidation, subsequent enhancement of diffusivity and bubble formation at grain boundaries, their interlinkage and release into open surfaces, was developed by Koo et al [13]. This model is stochastic in nature and incorporates inherent randomness of the underlying physical phenomenon using Monte Carlo method. While the prediction based on this model are in good agreement with the corresponding experimental measurements in the linear heating regime, strong under-predictions have been reported for the remaining regime. The Ivanov's model [14] gave good description of various processes involved in the release of FPs from the porous ceramic fuel, its leakage from clad and mixing with the primary coolant. Theoretical predictions based on this model have been reported in good agreement with the corresponding experimental data.

Combined failures based model has been developed by Clink and Freeburn [15] which was employed in an on-line coolant activity monitoring system. Such systems carryout estimation of failed fuel fractions in non-destructive manner. Normally, these systems are designed for constant power, steady state operational conditions. The Clinck and Freeburn model was observed to under-predict failed fuel fractions even for steady-state operation [16].

A theoretical model has been developed by Tucker and white [17] for the estimation of FPs from ceramic $\mathrm{UO}_{2}$ fuel. In this model, first, the probabilities of leakages of FPs from fuel interior through grain-edge tunnel pore to outer portions are figured out. These probabilities strongly depend on the interconnectivity of pores in the ceramic fuel. A good agreement has been reported between theoretical predictions made by using this model and the corresponding experimental measurements.

\section{FPA simulation codes}

In view of the importance of the FPA for normal operation as well as for accidental scenarios, various computer programs have been developed for its estimation. They fall into two basic categories:

- $\quad$ Point depletion codes

- Fission Product Transport Codes

- Empirical

- Semi-Empirical

- Mechanistic

The point depletion codes carryout production, buildup, decay and depletion calculations for a wide variety of radionuclides in the core region. As such, they provide reliable estimates of radioisotope inventory in the reactor fuel. They typically ignore spatial details while retaining spectral details of the neutron field. The widely used WIMS computer code [18] for 1-D transport theory macroscopic group constant generation employs 69-group library along with DSN or Stochastic methodology. It performs details buildup, depletion and burnup calculations for 35 distinct fission products along with one pseudo, lumped fission product. The WIMS code does not perform any further radionuclide transport calculations. The CASMO-4 [19] and DRWIN [20] also belong to the same pin/cell based macroscopic group constant generation codes as WIMS and as far as fission products are concerned, they are limited to radionuclide inventory calculations for the fuel region.

The ORIGEN2 computer code [21] provides extensive radionuclide inventory calculations for 950 fission products along with 120 actinides in point-wise buildup and depletion manner. While one can manually remove or add radionuclides in refueling options, no 
attempt is made in the code for the radionuclide transport calculations. An evolved version called MONTEBURNS [22] incorporates spatial details in the depletion/buildup calculations by coupling the ORIGEN2 code with the multipurpose radiation transport code MCNP [23].

The radionuclide transport code category is comprised of three types of computer codes: empirical, semi-empirical and mechanistic codes. In the empirical codes, various data fitting techniques are used for development of empirical models from detailed experimental observations. One advantage of this strategy is that no prior knowledge is required regarding the details of the underlying physical processes involved. At the same time, it gives most accurate results in the sense that they match the experimental results. Consequently, they are extensively used in risk assessment and safety analysis. Lumping of parameters and grouping of similar elements simplifies many features of these codes and adds to their computational efficiency. The MELCOR [24] and CORSOR [25] codes belong to the empirical radionuclide transport class of computer programs. While being highly efficient and reliable, the empirical codes are valid only in a limited range of parameters.

The limitations of the empirical models are relaxed somewhat by incorporating detailed modeling for a part of the simulation while the remaining part is attempted by using empirical approach. The FIPREM [26] computer code attempts fission product transport problem by using empirical Booth equivalent sphere model while detailed diffusion theory based finite difference model is employed for fission product transport into gap region.

The VICTORIA [27] and ECART [28] computer codes, being mechanistic in nature, do not face strict limits of validation. They carryout simulation of radionuclide transport in much broader range of accidental scenarios starting from releases, to dispersion and subsequent deposition. Since these computer programs were specifically designed for accident analysis, therefore, they cannot be used in normal steady-state or in transient cases.

Most of the available computer programs for transport analysis of fission product activity are focused on accidental analysis. For the analysis of fission product transport in the steady state and in transient analysis FPCART-ST computer code has been developed. The details regarding the mathematical modeling, computer implementation and results of simulations carried out using this code are provided here.

\section{Kinetic modeling}

In these work, a $300 \mathrm{MW}(\mathrm{e})$ PWR has been considered with design specifications as provided in Table 1. The primary circuit of a typical PWR with various indicated essential components is shown in Fig. 1. The reactor is taken with zero levels of FPA in the primary circuit at the start $(t=0)$. The FPA levels in Fuel/Gap/Coolant=F/G/C is governed by the following set of ODEs:

For the fuel region:

$$
\frac{d N_{F, i}}{d t}=F Y_{i} P+\sum_{j-1}^{i-1} f_{i j} \lambda_{j} N_{F, j}-\left[\lambda_{i}+v_{i}+\sigma_{i} \phi\right] N_{F, i},
$$

for the gap region:

$$
\frac{d N_{G, i}}{d t}=v_{i} N_{F, i}+\sum_{j-1}^{i-1} f_{i j} \lambda_{j} N_{G, j}-\left[\lambda_{i}+D \epsilon_{i}+\sigma_{i} \phi\right] N_{G, i}
$$


and, for the coolant region:

$$
\frac{d N_{C, i}}{d t}=D \epsilon_{i} N_{G, i}+\sum_{j-1}^{i-1} f_{i j} \lambda_{j} N_{C, j}+\left[\lambda_{i}+Q \frac{\eta_{i}}{W}+\beta+\tau \sigma_{i} \phi+\frac{L}{W}\right] N_{C, i} .
$$

where, ' $\mathrm{i}$ ' indicates the isotope in the decay chain consisting of four isotopes: $i=1,2, \ldots, 4$. The values of various parameters used in these simulations are listed in Table 2.

In order to compute the saturation values of various radioisotopes in the fuel, gap and coolant regions one can use the following analytical results:

For coolant region:

$$
N_{C, i}^{s a t}=\left[D \epsilon_{i} N_{G, i}^{s a t}+\sum_{j-1}^{i-1} f_{i j} \lambda_{j} N_{C, j}^{s a t}\right] /\left[\lambda_{i}+\frac{Q}{m} \eta_{i}+\beta_{i}+\tau \sigma_{i} \phi+\frac{L}{m}\right],
$$

For gap region,

$$
N_{G, i}^{s a t}=\left[v_{i} N_{F, j}^{s a t}+\sum_{j-1}^{i-1} f_{i j} \lambda_{j} N_{G, j}^{s a t}\right] /\left[\lambda_{i}+D \epsilon_{i}+\sigma_{i} \phi\right]
$$

and for fuel region:

$$
N_{F, i}^{s a t}=\left[F Y_{i} P+\sum_{j-1}^{i-1} f_{i j} \lambda_{j} N_{F, j}^{s a t}\right] /\left[\lambda_{i}+v_{i}+\sigma_{i} \phi\right] .
$$

\begin{tabular}{ll}
\hline Parameter & Value \\
\hline Specific power (MWth/Kg. U) & 33 \\
Number of fuel assemblies & 194 \\
In-let coolant temperature $\left({ }^{\circ} \mathrm{C}\right)$ & 293 \\
Out-let coolant temperature $\left({ }^{\circ} \mathrm{C}\right)$ & 329 \\
Power density $\left(\mathrm{MWth} / \mathrm{m}^{3}\right)$ & 102 \\
Fuel pins (rods) per assembly & 264 \\
Fuel material & $\mathrm{UO}_{2}$ \\
Clad material & $\mathrm{Zilcoloy}$ \\
Lattice pitch $(\mathrm{mm})$ & 12.6 \\
Fuel pin outer diameter $(\mathrm{mm})$ & 9.5 \\
Coolant pressure $(\mathrm{MPa})$ & 15.5 \\
Coolant flow rate $(\mathrm{Mg} / \mathrm{s})$ & 18.3 \\
Linear heat rate $\left(\mathrm{kW} / \mathrm{m}^{3}\right)$ & 17.5 \\
Average enrichment $(\%)$ & 3.0 \\
Core height $(\mathrm{m})$ & 4.17 \\
Core diameter $(\mathrm{m})$ & 3.37 \\
\hline
\end{tabular}

Table 1. Design data of a typical pressurized water reactor [37] 


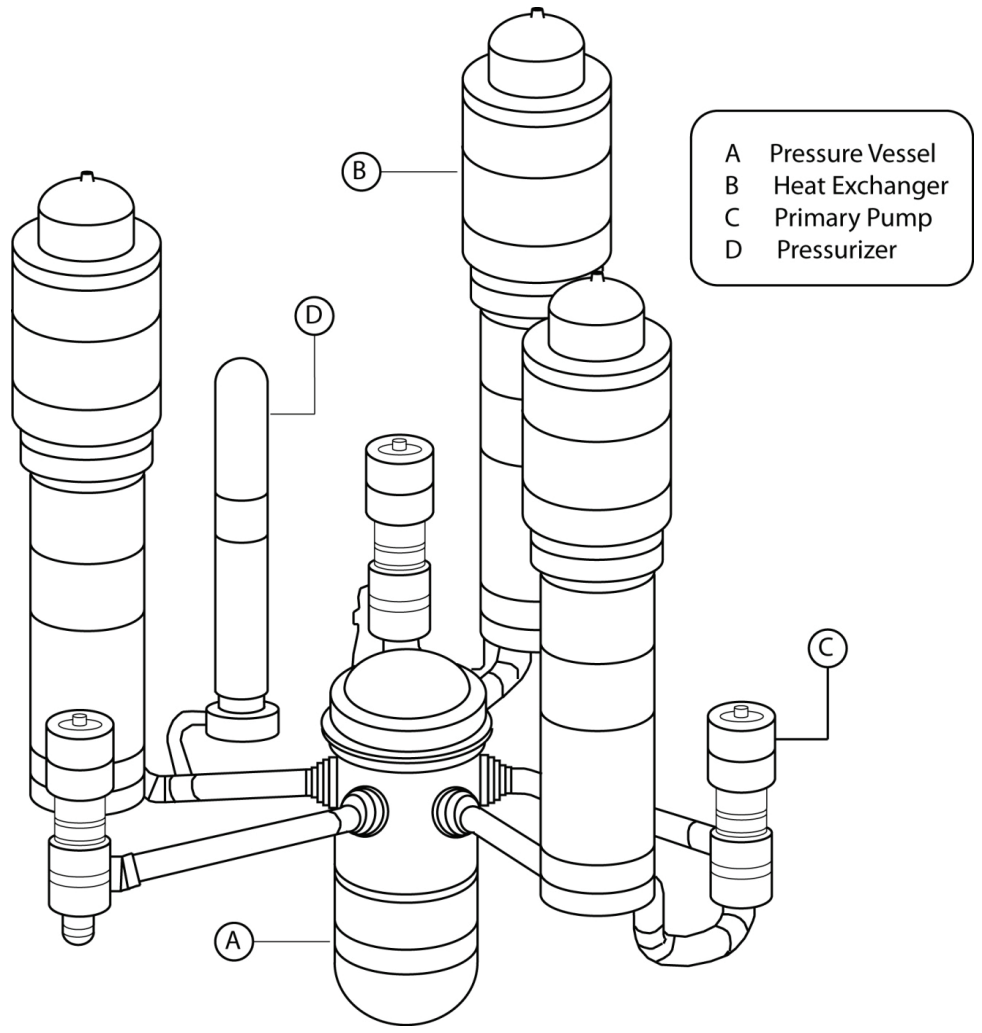

Fig. 1. A three dimensional perspective view of a typical PWR primary system with the pressure vessel, heat exchanger, primary pump and pressurizer indicated

\begin{tabular}{ll}
\hline Parameter & Value \\
\hline$V\left(\mathrm{~cm}^{3}\right)$ & $1.485 \times 10^{9}$ \\
$L(g / s)$ & 2.3 \\
$Q(g / s)$ & 470 \\
$D$ & $2.5 \times 10^{-3}$ \\
$W(g)$ & $1.072 \times 10^{9}$ \\
$\beta$ & 0.001 \\
$F($ Fissions $/ W . s)$ & $3.03 \times 10^{10}$ \\
$P_{o}\left(M W_{\text {th }}\right)$ & 998 \\
$\tau$ & 0.056 \\
\hline
\end{tabular}

Table 2. Values of different operational parameters used in simulations [37] 


\subsection{Deterministic computational methodology}

Various step involved in the transport of fission products, starting from their release in the fuel matrix, their transport from ceramic pores into the fuel-clad gap, their leakage from clad into the primary coolant, and subsequent removal by leakages, by filters, by radioactive decay etc., is depicted in Fig. 2. The FPA transport model has been implemented in the computer program FPCART. It uses LEOPARD [29] and ODMUG [30] programs as subroutines. The cell averaged multigroup group constant generation is carried out by the LEOPARD subroutine while the group fluxes are found by solution of one-dimensional diffusion equation in the ODMUG subroutine. In the FPCART code, the system of governing ODEs: Eqs. (1) upto (3) are solved numerically using Runge-Kutta (RK) method in this program. The RK-numerical provides efficient time domain solution yielding static as well as dynamic values of FPAs corresponding to about 50 different dominant fission products.

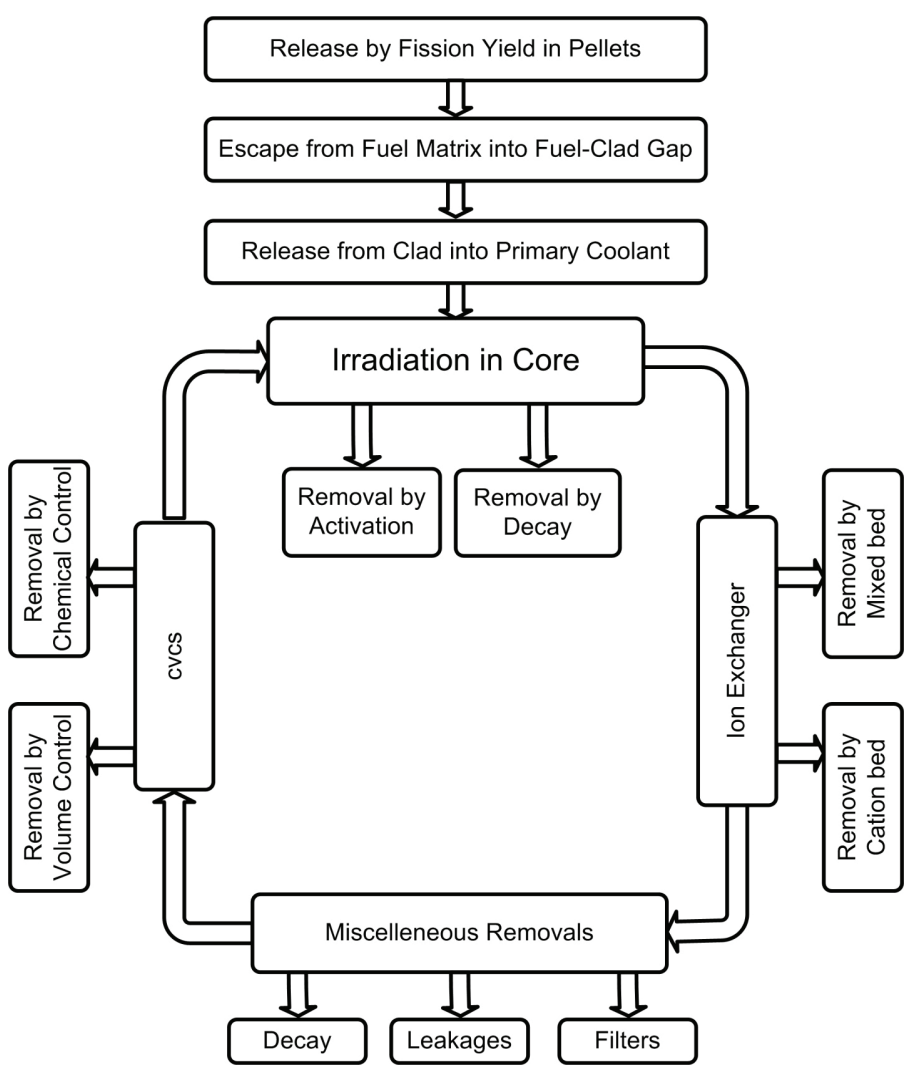

Fig. 2. Block diagram of the fission products production and removal mechanism in the primary circuit of a typical PWR

The computational cycle starts with initialization of the variables with $t=0$. The group constants are generated by the LEOPARD while the group flux are found using ODMUG. The values of FPAs in the fuel, gap and in primary coolant are initialized as zeros for the 
cold clean core. In the time loop, the values of FPAs for about 50 different radionuclides are calculated using RK-scheme for each next time step. The results are stored in separate data files for each fission product chain and for each region. The program allows performing these calculations for power as well as flow rate perturbations.

\subsection{Power perturbation model}

The FPCART computer code has built-in model for linear power perturbations. This model uses a rate parameter $\alpha$ representing the time rate of change of reactor power. Then, for a time range $\left[t_{i n}, t_{m}\right]$ the reactor power is calculated using:

$$
P(t)=P_{0} f(t)
$$

where,

$$
f(t)=\left\{\begin{array}{lr}
1, & t \leq t_{\text {in }} \\
1-\alpha\left[t_{m}-t\right], & t_{\text {in }} \leq t \leq t_{m} \\
w_{2} / w_{0} & t>t_{m}
\end{array}\right.
$$

Where

$\alpha$ is slope of the linear change of reactor power;

$t_{i n}$ is start of reactor power perturbation;

$t_{m}$ is end of the reactor power.

\subsection{Flow-rate perturbation model}

The flow rate perturbation involves primary pump modeling where the balance of angular momentum with the frictional deceleration yields [31] :

$$
l \rho \frac{d v}{d t}=-\frac{1}{2} C_{f} \rho v^{2}
$$

where, $l$ is the total length of the loop; $\rho$ is the fluid density; $C_{f}$ represents total pressure loss coefficient; and $v$ is the fluid speed. The Eq. (9) yields the corresponding solution as flow rate $w(t)$ is:

$$
w(t)=w_{0} /\left[1-t / t_{p}\right],
$$

where, $w_{0}$ represents the steady state value of flow rate; and $t_{p}=2 l /\left(C_{f} v_{0}\right)$ which is typically around $2000 \mathrm{~h}$ for transients without boiling crisis.

\section{Stochastic release model}

The release of fission products from fuel pins is essentially a random process as the time of clad failure, the amount of release as well as the duration of fission product release cannot be specified exactly beforehand. In order to model these aspects in more realistic manner, Monte Carlo based stochastic approach has been used in these simulations. The modified version FPCART-ST is primarily deterministic-stochastic hybrid code. The sampling of fuel pin failure probability distribution function $g(t)$ yields the fuel pin failure time sequence. The intensity function $\psi(t)$ is correspondingly: 


$$
\psi(t)=g(t) / G(t)
$$

where, the cumulative probability distribution $G(t)$ :

$$
G(t)=\exp \left(-\int_{0}^{t} g(s) d s\right)
$$

serves the normalization. According to the standard rejection technique [??] the probability of accepting a fuel failure at $t_{k}$ after $t_{j}$ is found by using a random number ' $\eta$ ' and comparing it with the ratio ' $q$ ':

$$
q=\frac{g\left(t_{k}\right)}{g\left(t_{j}\right)}
$$

and, if $\eta<q$, this step is repeated otherwise, $t_{k}$ is accepted as a fuel failure event time. The fuel matrix to gap escape rate coefficient takes the form:

$$
\epsilon=D_{0} \epsilon_{0} \exp \left[-\xi\left(t-t_{0}\right)\right]+D_{F} \epsilon_{0}
$$

where, $\epsilon_{0}$ is the starting value of burst release rate from a punctured fuel rod; $\xi$ represents the characteristic decay constant for the escape rate; $t_{0}$ is time at which the fuel rod fuel rod failure starts; $D_{F}$ represents the current number of failed fuel rods while $D_{0}=1$ is flag for the failure of the current fuel rod. Typical values of these parameters are: $\xi=7.2 \times 10^{-5} \mathrm{~s}^{-1}$ $\epsilon_{0}=10^{-8} s^{-1} ; D=60$

\section{Results and discussion}

\subsection{Buildup of fission products in steady-state}

The FPCART computer code has been used for the simulation of fission product buildup to steady state saturation values starting with a cold clean core. For a $300 \mathrm{MW}(\mathrm{e})$ typical PWR, the predictions of the FPCART program have been compared with the widely used ORIGEN2 computer code and excellent agreement between the corresponding values has been found. The observed small difference, of the order of a few percent only, can be attributed to difference in the yield of the fission products. The results are shown in Fig. 3. The results indicate dominance of ${ }^{131} \mathrm{I},{ }^{134} \mathrm{Te}$, $133 \mathrm{I}$ and ${ }^{135} \mathrm{I}$ in the saturation values of fission product activity in the fuel matrix.

\section{$5.2{ }^{135} \mathrm{Xe}$ activity under step and ramp power transients}

With largest absorption cross section, ${ }^{135} \mathrm{Xe}$ acts as dominant poison in nuclear reactors. At the start of operation, the ${ }^{135}$ Xe levels are zero which climb to saturation levels with time which depend on the power level and time behavior of reactor power during this period. FPCART simulations have been carried out for the study of ${ }^{135} \mathrm{Xe}$ transients for step and ramp power transients. The results are shown in Fig. 4 . The ramp power transients lead to somewhat slower rise to saturation levels as compared with the step power changes. For post-scram time periods, the ${ }^{135} \mathrm{Xe}$ levels rise to maximum values; which is followed by gradual decrease. 


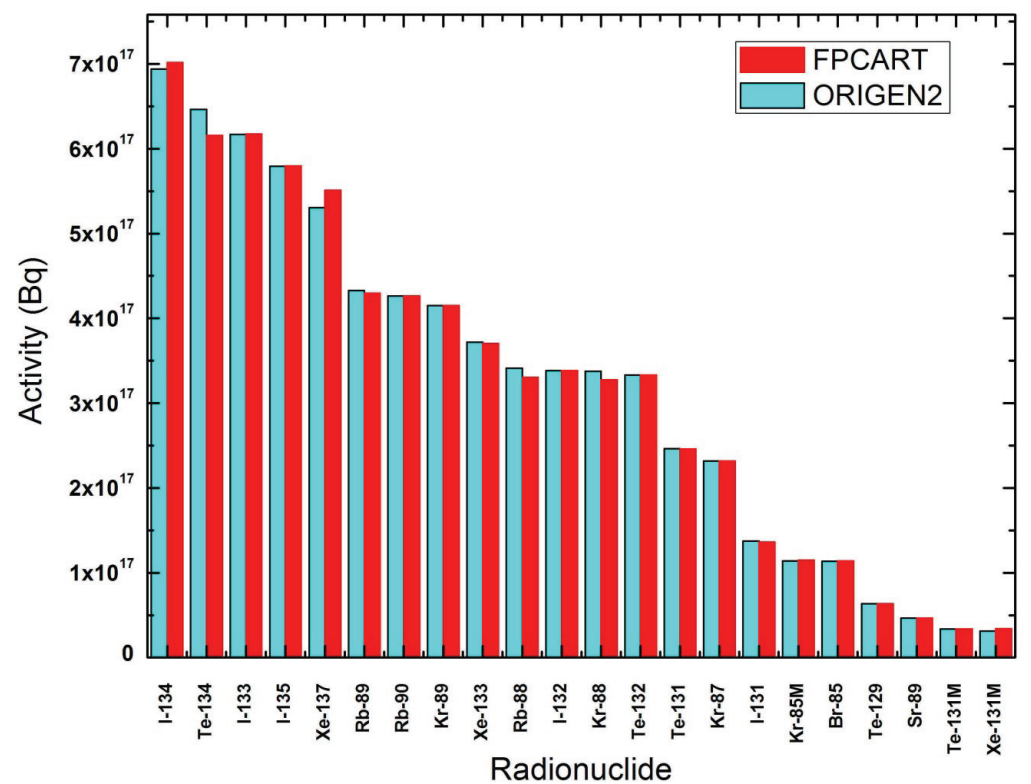

Fig. 3. For steady state operation, FPCART predicted saturation values of activities of various isotopes in PWR fuel with the corresponding computed data using the ORIGEN2 code [35]

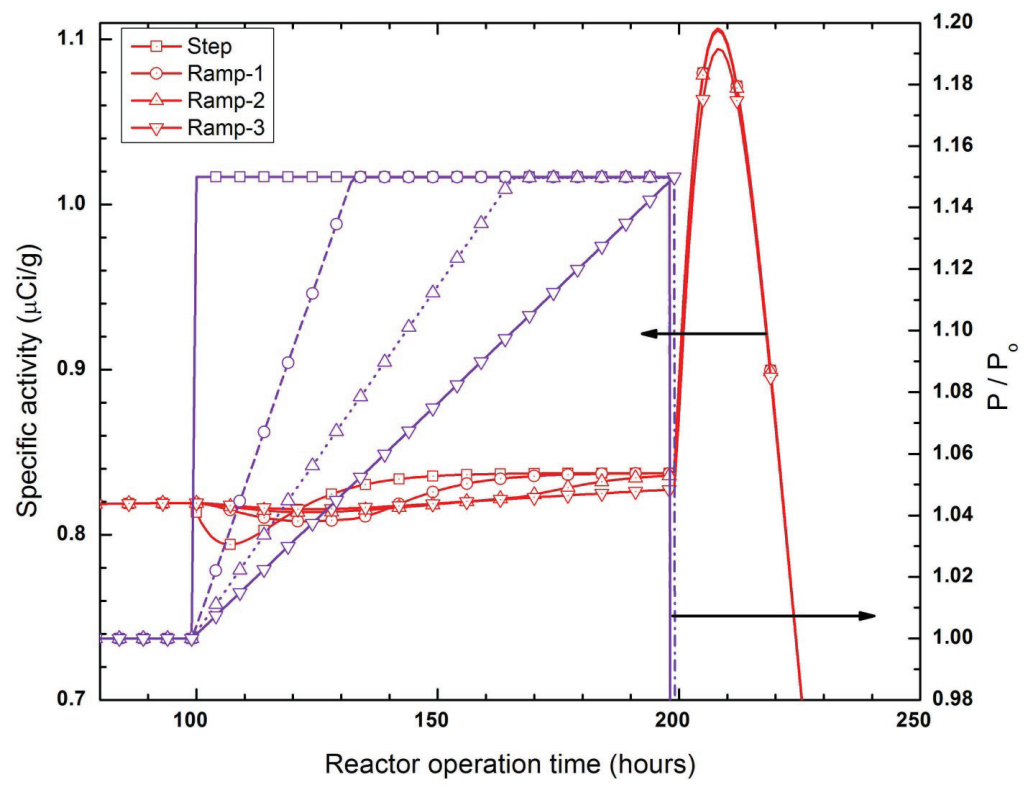

Fig. 4. FPCART simulated variation of ${ }^{135}$ Xe specific activity with time for step and ramp power transients 


\subsection{Fission product activity under pump coast-down}

The pump-coast down belongs to general class of flow rate transients. During these transients, the core residence time, and total circuit time along with the effective neutron flux values are influenced by the change in flow rate. A decrease in flow rate leads to increase in the fission product activity values. In this study, fifteen different radionuclides belonging to fission products and their decay chains were selected and their approach towards saturation levels was studied under constant power. The pump coast-down was initiated when the levels reached sufficiently close to saturation levels. The corresponding results are shown in Fig. 5 where the isotope-wise as well as total activity variations are shown after the pump coast-down. It is observed that ${ }^{133} \mathrm{Xe}$ is the main contributor having

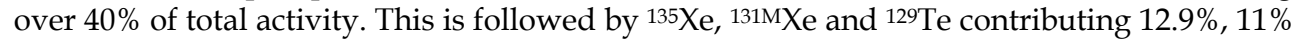
and $8.2 \%$ of the total activity respectively. During the pump coast-down period, the total activity level raises well over $8.6 \%$ level before the loss-of-flow signals the reactor shutdown.

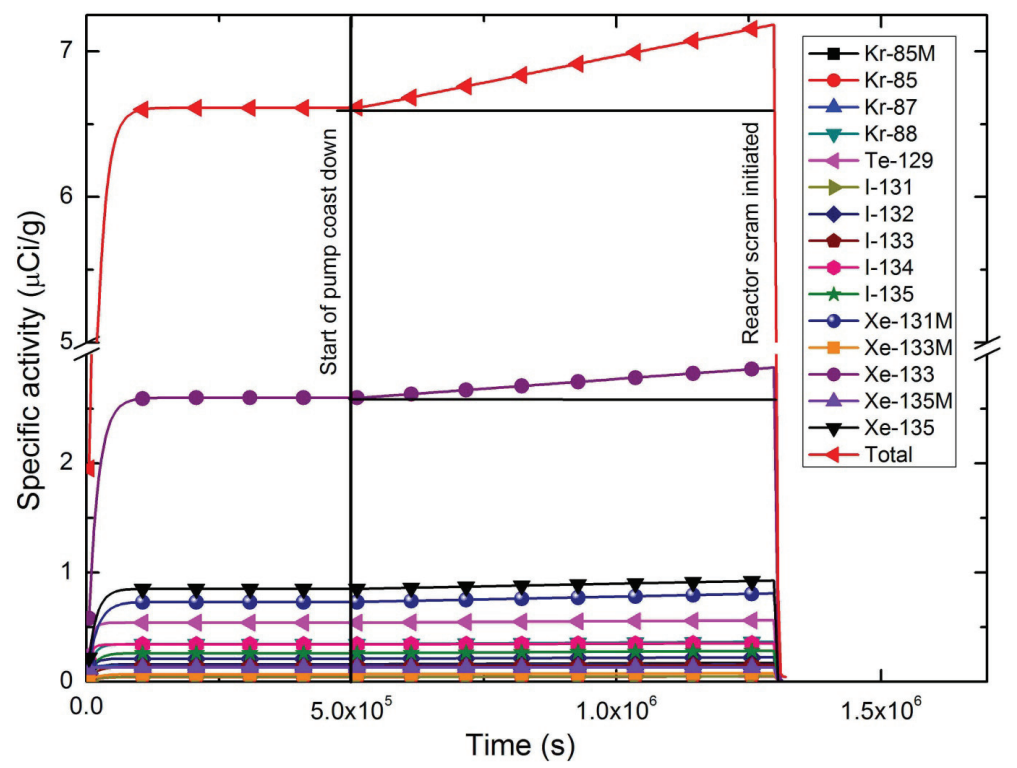

Fig. 5. FPCART simulated primary coolant total activity due to fission products of a 1000 MWth PWR for a $t_{p}=2000 \mathrm{~h}$ pump coast-down flow rate transient [36]

\subsection{FPCART simulations of FPA under power transients}

For validation of the three stage deterministic computational methodology of the FPCART computer code, its predictions were compared against actual experimental data. In the case of BEZNAU (Unit 1) [32] , the FPCART computed time variation of ${ }^{131}$ I for various power variations during the first cycle have been compared with the corresponding experimental measurements. It is clear from Fig. 6 that FPCART predictions are in good agreement with the experimental data throughout time range. A similar trend has been observed in the case of ${ }^{131}$ I activity in the ZORITA [32] power plant where again the FPCART predictions have been found in good agreement with the corresponding experimental measurements as shown in Fig. 7. 


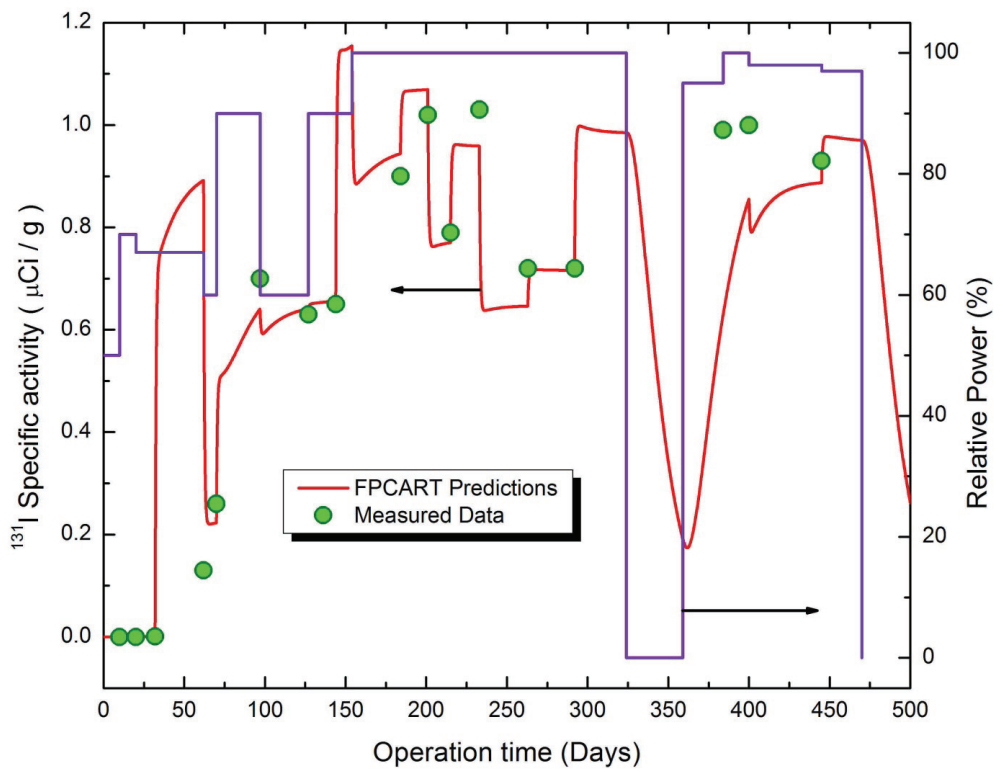

Fig. 6. For power transients, FPCART predicted values of 131I specific activity variations with time compared with the corresponding experimental data for the BEZNAU (Unit-1) power plant

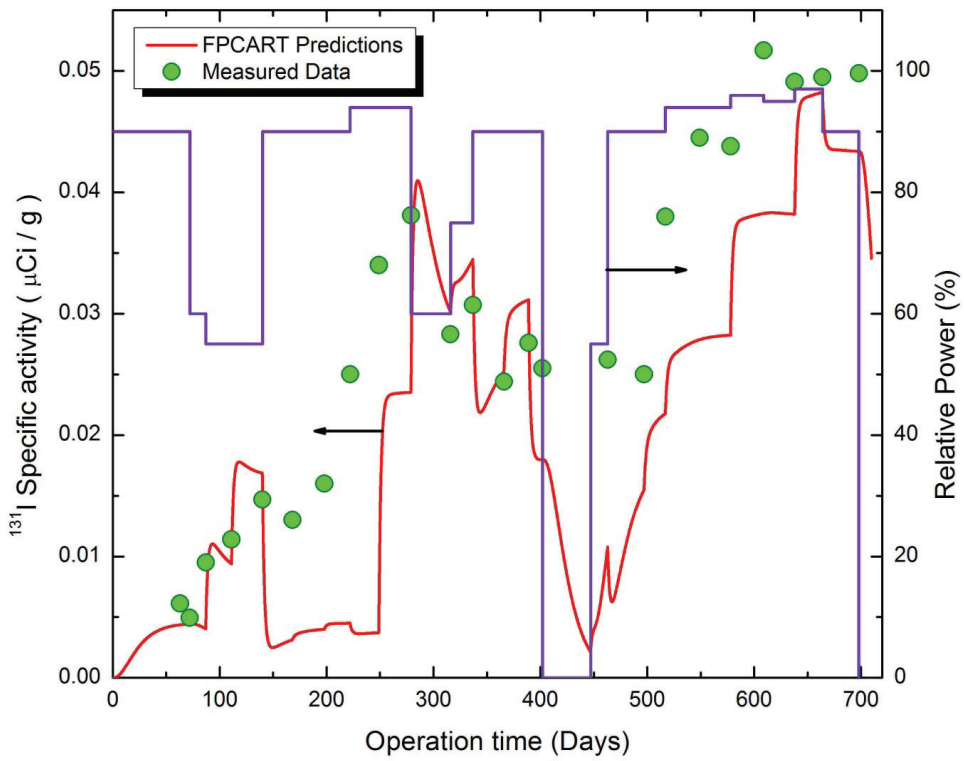

Fig. 7. For power transients, FPCART predicted values of 131 I specific activity variations with time compared with the corresponding experimental data for the ZORITA power plant 


\subsection{Stochastic simulations of FPA}

A typical PWR has large number of fuel pins arranged in fuel bundles. They can fail at any time due to a wide variety of causes/ reasons. Their failure is essentially a random phenomenon. Therefore, stochastic techniques are well suited for the failure modeling. In this work, a time sequence of their failure has been generated by sampling time dependent intensity function. The escape of fission product and their siblings have been modeled using three step deterministic model of FPCART.

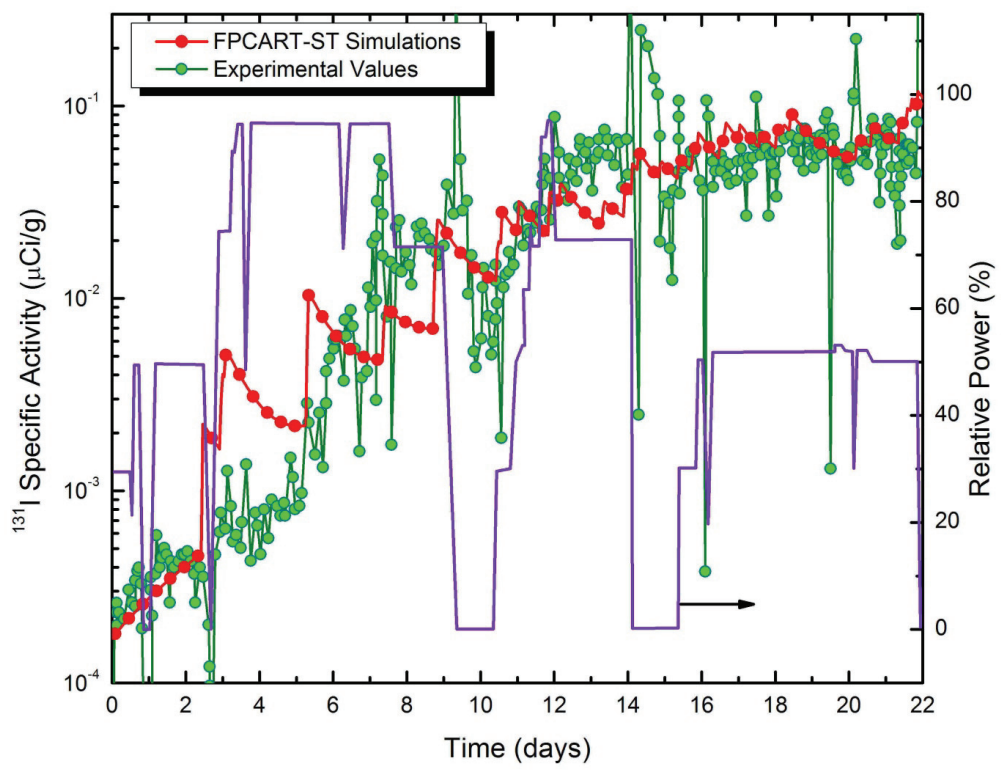

Fig. 8. For power transients, FPCART predicted values of ${ }^{131}$ I specific activity variations with time compared with the corresponding experimental data for the ANGRA-1 power plant [37]

The Westinghouse designed ANGRA-1 [33] (657 MWe) nuclear power plant was shut-down prematurely in the $4^{\text {th }}$ cycle due to abnormally high levels in the primary coolant. As suspected, one sixth of its core had failed resulting in leakage of fission products and their daughters into the primary coolant stream. The first 22 days of this event have been simulated using the stochastic FPCART-ST computer code. For ${ }^{131} \mathrm{I}$ activity, the predicted values for $70 \%$ power levels remain within $15 \%$ from the corresponding experimental measurements. Keeping in view the complex nature of the event being simulated, the predictions show good agreement. For larger variations in power level, deviations are found in the predictions which may be attributed to variations in the flow rate that have not been included in this model. The ${ }^{131}$ I activity spikes found in experimental data remain smeared in the predicted data requiring further investigation regarding couples flow-rate \& power transients.

The EDITHMOX [34] experiments, given in the OECD/NEA/IAEA IFPE database, were considered for validation of the FPCART-ST computer code. These experiments are part of a broad experimental program conducted a the France's Siloe research reactor. The EDITHMOX experiment was conducted at the Jet Pompe water loop. Release rates of 
various fission products were studied for mixed-oxide fuel under various conditions of burnup and operating power. The FPCART-ST predicted time variation of $85 \mathrm{Kr}$ activity for various power levels has been found in good agreement with the corresponding experimental data available from the EDITHMOX experiment. As shown in Fig. 9, the agreement prevails over most of the measured time while some deviations are observed for the first large power drop. This may be attributed to coupling of power transients with flowrate perturbations which has been ignored in these simulations.

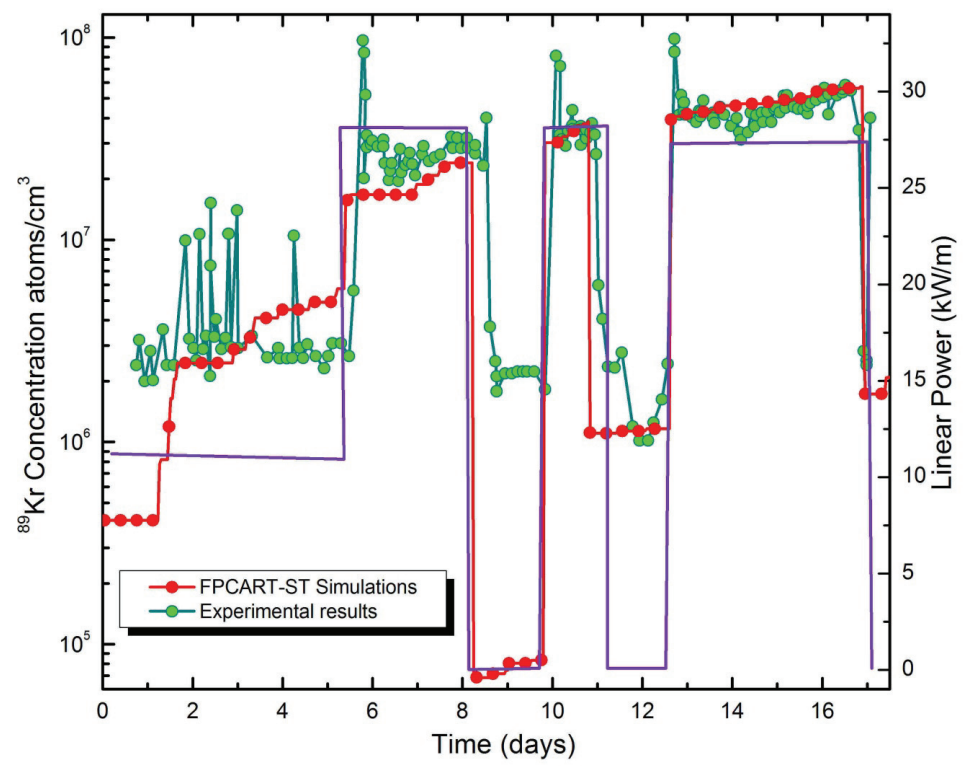

Fig. 9. For power transients, FPCART predicted values of ${ }^{89} \mathrm{Kr}$ specific activity variations with time compared with the corresponding experimental data for the EDITHMOX-1 (Unit-1) power plant [37]

\section{Conclusions}

The normal operation as well as during the accidental situations, the fission products and their daughters play a dominant role toward potentially imparting high levels of exposure to radiation workers and general public. A review of research effort devoted to modeling and simulation of fission product activity in the primary circuits of typical PWRs has been presented in this work. While the mechanistic models have been found superior in the context of the range of applicability against the empirical and semi-empirical models, the available computer codes are limited to accidental release modeling generally.

Development of methodology for estimation of FPA levels in the primary loops of PWRs during normal steady-state as well as transient conditions has been carried out in this work. The aim of this effort is to model FPA releases into primary coolant in the steady state, as well as in power and flow-rate transients. For this purpose multi-step model has been presented in this work that tracks fission product transport from fuel to fuel-clad gap and finally to the primary coolant. The influence of filters, ion-exchanges, leakages, decay etc. 
has been incorporated in this model. For randomly failing fuel pins, stochastic modeling has been carried out for burst releases. The coupled deterministic-stochastic hybrid approach has been found effective for large scale fuel failure events.

The trends towards core life-time extensions and high burn-up cores, coupled with aging of the existing fleet of nuclear reactors; it has become imperative to limit fission product activity to much lower levels. This is required in order to keep PWRs economically feasible against their competitors.

\section{Nomenclature}

$\begin{array}{ll}\text { Symbol } & \text { Represents } \\ N_{X, i} & \text { Number of } \mathrm{i}^{\text {th }} \text { radionuclide atoms in the region: } \\ \phi & \text { Fuel }(\mathrm{X}=\mathrm{F}), \mathrm{Gap}(\mathrm{X}=\mathrm{G}) \text { and Coolant }(\mathrm{X}=\mathrm{C}) \\ \eta_{i} & \text { Neutron flux }\left(\# / \mathrm{cm}^{2} . \mathrm{s}\right) \\ Q & \text { Resin purification efficiency for the } \mathrm{i}^{\text {th }} \text { radionuclide } \\ v_{i} & \text { Let-down flow rate }(\mathrm{g} / \mathrm{s}) \\ \sigma_{i} & \text { Escape rate coefficient of the } \mathrm{i}^{\text {th }} \text { radionuclide } \\ Y_{i} & \text { Microscopic absorption cross section for the } \mathrm{i}^{\text {th }} \text { radionuclide } \\ f_{i j} & \text { Fission yield of the } \mathrm{i}^{\text {th }} \text { radionuclide } \\ \beta & \text { Branching ratio }(i \rightarrow j) \text { in the } \mathrm{i}^{\text {th }} \text { chain } \\ L & \text { Bleed-out fraction of the primary coolant for boron chemical control } \\ W & \text { The coolant leakage rate }(\mathrm{g} / \mathrm{s}) \\ \lambda_{i} & \text { The total primary coolant mass }(\mathrm{g}) \\ F & \text { Decay constant of the } \mathrm{i}^{\text {th }} \text { radionuclide }\left(\mathrm{s}^{-1}\right) \\ P & \text { Average fission rate (fissions } / \mathrm{W} . \mathrm{s}) \\ D & \text { Thermal power of reactor }(\mathrm{W}) \\ \tau & \text { Failed fuel fraction (\#) } \\ D_{F} & \text { Core-to-circuit primary coolant resident time ratio }(\#) \\ \xi & \text { Number of failed fuel rods }\end{array}$

\section{References}

[1] IAEA, Nuclear power reactors in the world, Reference Data Series No. 2, International Atomic Energy Commission, IAEA-RDS-2/30, Vienna, 2010.

[2] S. Rippon, History of the PWR and its worldwide development, Energy Policy, 12(3), (1984) 259-265.

[3] C. J. Wood, Recent developments in LWR radiation field control, Progress in Nuclear Energy, 19(3), (1987), 241-266.

[4] G. C. Comley, The significance of corrosion products in water reactor coolant circuits, Progress in Nuclear Energy, 16(1), (1985) 41-72. 
[5] D. H. Lister, Corrosion-product release in Light Water Reactors, EPRI Report NP-3460, March (1984).

[6] T. Moore, The challenge of nuclear fuel reliability, EPRI, J. (Fall) (2005).

[7] H. Zänker, H. Müller, R. Berndt, A complementary cesium coolant concentration ratio for localizing defective PWR fuel rods during reactor operation, J. Radioanalytical and Nuclear Chemistry, 152(1), (1991) 47-54.

[8] G. F. Huang, L. L. Tong, J. X. Li, X. W. Cao, Study on mitigation of in-vessel release of fission products in severe accidents of PWR, Nuclear Engineering and Design, 240(11), (2010) 3888-3897.

[9] Y. Pontillon, G. Ducros, P. P. Malgouyres, Behaviour of fission products under severe PWR accident conditions VERCORS experimental programme-Part 1: General description of the programme, Nuclear Engineering and Design, 240(7) (2010) 18431852.

[10] K. Y- Hyun, S. D- Seong, Y. Y- Ku, An analysis method for the rod gap inventory of unstable fission products during steady-state operation, J. Nuclear Materials, 209(1) (1994) 62-78.

[11] B. J. Lewis, A generalized model for fission product transport in the fuel-to-sheath gap of defective fuel elements, J. Nucl. Materials, 175(3) (1990) 218-226.

[12] M. Barrachin, R. Dubourg, M. P. Kissane, V. Ozrin, Progress in understanding fissionproduct behavior in coated uranium-dioxide fuel particles, J. Nucl. Materials, 385(2) (2009) 372-386.

[13] Y. H. Koo, D. S. Sohn, Y. K. Yoon, Release of unstable fission products from defective fuel rods to the coolant of a PWR, J. Nuclear Materials, 209(3), (1994) 248-258.

[14] A. S. Avanov, The model of the fission gas release out of porous fuel, Annals of Nuclear Energy, 25(15), (1998) 1275-1280.

[15] L. Clink, H. Freeburn, Estimation of PWR fuel rod failure throughout a cycle, Trans. Am. Nucl. Soc., 54(1), (1987), 13.

[16] J. D. B. Lambert, K. C. Gross, E. V. Depiante, Adaptation of gas tagging for failed fuel identification in light water reactors, Proc. ASME $4^{\text {th }}$ Intl. Conf. Nuclear Engineering. CONF-960306-25, (1996).

[17] M. O. Tucker, R. J. White, The release of fission products from $\mathrm{UO}_{2}$ during irradiation, J. Nuclear Materials, 87(1), (1979) 1-10.

[18] M. J. Halsall, A summary of WIMSD4 input options, Atomic Energy Establishment, Winfrith, Dorchester AEEW-M-1327, (1980).

[19] D. Knott, B. H. Forssén, M. Edenius, CASMO-4, A fuel assemblyburn-up program methodology, STUDSVK/SOA-95/2, (1995).

[20] A. Tsilanizara, DARWIM: An evolution code system for a large range of applications, J. Nucl. Sci. Technol, 37, (2000) 845-849.

[21] A. G. Groff, ORIGEN2: A versatile computer code for calculating the nuclide compositions and characteristics of nuclear materials, Nucl. Technol., 62 (1983) $335-352$.

[22] W. S. Charlton, R. T. Perry, B. L. Fearey, T. A. Parish, Calculated actinide and fission product concentration ratios for gaseous effluent monitoring using MONTEBURNS 3.01, Nucl. Technol., 131 (2000) 210-227.

[23] J. F. Briesmeister, (ed.), MCNP-A general Monte Carlo N-particle transport code, User's manual: Los Alamos National Laboratory, LA-13709-M, (2000). 
[24] R.M. Summers, et al., MELCOR 1.8.0: A Computer Code for Nuclear Reactor Severe Accident Source Term and Risk Assessment Analyses, NUREG/CR-5531, SAND900363 (1991).

[25] M. R. Kuhlmann, D. J. Lehmicke, R. O. Meyer, CORSOR user's manual, Battelle Columbus Labs. USNRC Report NUREG/CR-4173, (1985).

[26] C. Ronchi and C. T. Walker, Determination of Xenon Concentration in Nuclear Fuels by Electron Microbe Analysis, J. Phys. D: Applied Physics, 13, (1980) 2175.

[27] T. J. Haemes, D. A. Williams, N. E. Bixler, A. J. Grimley, C. J. Wheatley, N. A. Johns, N. M. Chown, VICTORIA: A mechanistic model of radionuclide behavior in the reactor coolant system under severe accident conditions, Sandia National Laboratory, Albuqurque, NM 87185, NUREG/CR-5545, (1990).

[28] F. Parozzi, "Computer Models on Fission Product and Aerosol Behavior in the LWR Primary System, Part II," Commission of the European Communities, EUR Report \# 14676 EN, (1992).

[29] R. F. Barry, LEOPARD: A Spectrum Dependent Non-Spatial Depletion Code for IBM7094, WCAP-3269-26, Westinghouse Electric Corporation (1963).

[30] Thomas, J. R., Edlund, H. C., Reactor Statics Module - Multigroup Criticality Calculations, Proc. Conf. ICTP, Trieste (1980).

[31] E. E. Lewis, Nuclear power reactor safety, John Wiley \& Sons, (1977).

[32] WEC, Source term data for Westinghouse Pressurized Water Reactors, Westinghouse Electric Corporation, Pittsburg, Pennsylvania WCAP-8253, May (1974).

[33] IAEA, Fuel failure in water reactors: Causes and Mitigation, IAEA-TECDOC-1345, June (2002).

[34] C. Baud, D. Parrat, CEA-DMECN, Complete rendu DTP/SECC No. 41/93, Center d'Etudes Nucleaires de Grenoble, (1982).

[35] M. J. Iqbal, N. M. Mirza, S. M. Mirza, Kinetic simulation of fission product activity in primary coolant of typical PWRs under power perturbations, Nuclear Engineering and Design 237(2), (2006) 199-205.

[36] S. M. Mirza, M. J. Iqbal, N. M. Mirza, Effect of flow rate transients on fission product activity in primary coolant of PWRs, Progress in Nuclear Energy, 49 (2007) 120 129.

[37] M. J. Iqbal, N. M. Mirza, S. M. Mirza, Stochastic simulation of fission product activity in primary coolant due to fuel rod failures in typical PWRs under power transients, J. Nuclear Materials, 372 (2008) 132-140. 


\title{
Thermal-Hydraulic Simulation of Supercritical-Water-Cooled Reactors
}

\author{
Markku Hänninen and Joona Kurki \\ VTT Technical Research Centre of Finland \\ Tietotie 3, Espoo, FI-02044 VTT, \\ Finland
}

\section{Introduction}

At supercritical pressures the distinction between the liquid and gas phases disappears, and any fluid stays in a single continuous phase: no evaporation or condensation is observed. At supercritical state the thermo-physical properties of the fluid, such as density and viscosity, change smoothly from those of a liquid-like fluid to those of a gas-like fluid as the fluid is heated. Because of the single-phase nature, a one-phase model would be ideal for thermalhydraulic simulation above the critical pressure. However, in the nuclear power plant applications the one-phase model is not sufficient, because in transient and accident scenarios, the pressure may drop below the critical pressure, turning the coolant abruptly from a one-phase fluid into a two-phase mixture. Therefore the thermal-hydraulic model has to be able to reliably simulate not only supercritical pressure flows but also flows in the two-phase conditions, and thus the six-equation model has to be used.

When the six-equation model is applied to supercritical-pressure calculation, the questions how the model behaves near and above the critical pressure, and how the phase transition through the supercritical-pressure region is handled, are inevitably encountered. Above the critical pressure the latent heat of evaporation disappears and the whole concept of phase change is no longer meaningful. The set of constitutive equations needed in the six-equation solution including friction and heat transfer correlations, has been developed separately for both phases. The capability of constitutive equations, and the way how they are used above the supercritical pressure point, have to be carefully examined.

In this article, the thermal hydraulic simulation model, which has been implemented in the system code APROS, is presented and discussed. Test cases, which prove the validity of the model, are depicted. Finally, the HPLWR concept is used as a pilot simulation case and selected simulation results are presented.

\section{Modeling of supercritical fluid thermal hydraulics}

One possibility to maintain separate liquid and gas phases in the supercritical flow model is to use a small evaporation heat, and then apply the concept of the pseudo-critical line. The pseudo-critical line is an extension of the saturation curve to the supercritical pressure region: it starts from the point where the saturation curve ends (the critical point), and it can 
be thought to approximately divide the supercritical pressure region to sub-regions of pseudo-liquid and pseudo-gas. The thermo-physical properties of water and steam undergo rapid changes near the pseudo-critical line and therefore the quality and accuracy of the steam tables is essential in calculation of flows under supercritical conditions. One difficult problem is related to the heat transfer at supercritical pressures; if the ratio of the heat flux to mass flux exceeds certain value and flow is directed upwards, the heat transfer rate may suddenly be reduced, and remarkable heat transfer deterioration may occur. The same phenomena may occur due to flow acceleration. The present heat transfer correlations are not able to predict properly this phenomenon. However, in conditions where this heat transfer impairment does not occur, heat transfer rates can be predicted with a reasonable accuracy using the currently-available correlations. Another issue that has to be taken into account in thermal hydraulic simulations of SCW reactors is the possible appearance of flow instabilities. Similarly to the boiling water reactors, instabilities in the core of SCWR may appear when the ratio of the heat flux to mass flux exceeds a certain value.

\section{Solution principles of the six-equation model}

At present, the system-scale safety analyses of nuclear power plants are generally calculated using the six-equation flow model. The safety analyses conducted for a particular nuclear power plant include mainly different loss-of-coolant scenarios, where the pressure in the primary circuit decreases at a rate depending on the size of the break. This means that also in the case of supercritical-water-cooled reactors, boiling may occur during accident conditions, and therefore also the simulation tools used for the safety analyses of the SCWR's have to be able to calculate similar two-phase phenomena as in the present nuclear reactors. A practical way to develop a supercritical pressure safety code, is to take a present code and modify it to cope with the physical features at supercritical pressures.

The six-equation model of APROS used for the two-phase thermal hydraulics is based on the one-dimensional partial differential equation system which expresses the conservation principles of mass, momentum and energy (Siikonen 1987). When these equations are written separately for both the liquid and the gas phase, altogether six partial differential equations are obtained. The equations are of the form

$$
\begin{gathered}
\frac{\partial\left(\alpha_{k} \rho_{k}\right)}{\partial t}+\frac{\partial\left(\alpha_{k} \rho_{k} u_{k}\right)}{\partial z}=\Gamma_{k}, \\
\frac{\partial\left(\alpha_{k} \rho_{k} u_{k}\right)}{\partial t}+\frac{\partial\left(\alpha_{k} \rho_{k} u_{k}^{2}\right)}{\partial z}=\Gamma_{k} u_{\mathrm{i} k}+\alpha_{k} \rho_{k} \vec{g}+F_{\mathrm{w} k}+F_{\mathrm{i} k}, \\
\frac{\partial\left(\alpha_{k} \rho_{k} h_{k}\right)}{\partial t}+\frac{\partial\left(\alpha_{k} \rho_{k} u_{k} h_{k}\right)}{\partial z}=\alpha_{k} \frac{\partial p}{\partial t}+\Gamma_{k} h_{\mathrm{i} k}+q_{\mathrm{i} k}+F_{\mathrm{w} k} u_{k}+F_{\mathrm{i} k} u_{\mathrm{i} k} .
\end{gathered}
$$

In the equations, the subscript $k$ is either 1 (= liquid) or $g$ (= gas). The subscript $\mathrm{i}$ refers to interface and the subscript $\mathrm{w}$ to the wall. The term $\Gamma$ is the mass change rate between phases (evaporation as positive), and the terms $F$ and $q$ denote friction force and heat transfer rate. For practical reasons, the energy equation (3) is written in terms of the total enthalpy, which equals to conventional "static" enthalpy plus all the kinetic energy: $h=h_{\text {stat }}+1 / 2 \mathrm{u}^{2}$. 
The equations are discretized with respect to space and time and the non-linear terms are linearized in order to allow the use of an iterative solution procedure (Hänninen \& Ylijoki). For the spatial discretization a staggered scheme has been applied, meaning that mass and energy balances are solved in one calculation mesh, and the momentum balances in another. The state variables, such as pressure, steam volume fraction, as well as enthalpy and density of both phases, are calculated in the centre of the mass mesh cells and the flow related variables, such as gas and liquid velocities, are calculated at the border between two mass mesh cells. In solving the enthalpies, the first order upwind scheme has been utilized normally. In the mesh cell, the quantities are averaged over the whole mesh, i.e. no distribution is used.

In the case of the APROS code, the main idea in the solution algorithm is that the liquid and gas velocities in the mass equation are substituted by the velocities from the linearized momentum equation. In the momentum equation the linearization has been made only for the local momentum flow. For the upwind momentum flows the values of the previous iteration is used. In addition the phase densities are linearized with respect to pressure. The density is linearized as

$$
\rho_{k}^{n}=\rho_{k}+\frac{\partial \rho_{k}}{\partial p}\left(p^{n}-p\right),
$$

where the superscript $n$ refers to the value at the new time step. When this linearization is made together with eliminating phase velocities with the aid of the linearized momentum equation, a linear equation group, where the pressures are the only unknown variables is formed. Solution of this equation system requires that the derivatives of density are always positive, and also the phase densities obtained from the steam table are increasing with increasing pressure.

In the one-dimensional formulation, phenomena that depend on gradients transverse to the main flow direction, like friction and heat transfer between the gas and liquid phases, and between the wall and the fluid phases, have to be described through constitutive equations, which are normally expressed as empirical correlations. These additional equations are needed to close the system formed by six discretized partial differential equations.

\section{Application of the six-equation model to supercritical-pressure flow}

When the six-equation model is applied to supercritical-pressure calculation, the problems how the model behaves near and above the critical pressure, and how the phase transition needed in two-phase model is handled when the pressure exceeds the supercritical line, are inevitably encountered. Above the critical pressure the heat of evaporation disappears and the whole concept of phase change is no longer meaningful. The set of constitutive equations needed in the six-equation solution includes friction and heat transfer correlations that are developed separately for both phases. Also, many of the material properties exhibit sharp changes near the pseudo-critical line, and therefore correlations developed for subcritical pressures cannot give sensible values, and thus special correlations developed for supercritical pressure region have to be used instead. Then, how the transition from the subcritical to supercritical takes place, has to be taken into account in developing the correlation structure for friction and heat transfer. The capability of constitutive equations and the way how they are used has to be carefully examined (Hänninen \& Kurki, 2008). 
In system codes, the thermo-physical properties of the fluids are often given as tabulated values as a function of pressure and enthalpy. Especially for values near the critical point, a very dense network of the tabulated pressure and enthalpy points is needed in order to ensure the accuracy of the used property values. The heat capacities of the liquid and steam approach simultaneously infinity as the latent heat of evaporation approaches zero. In addition, the density and viscosity experience the sharp changes near the critical line (see Figure 1).
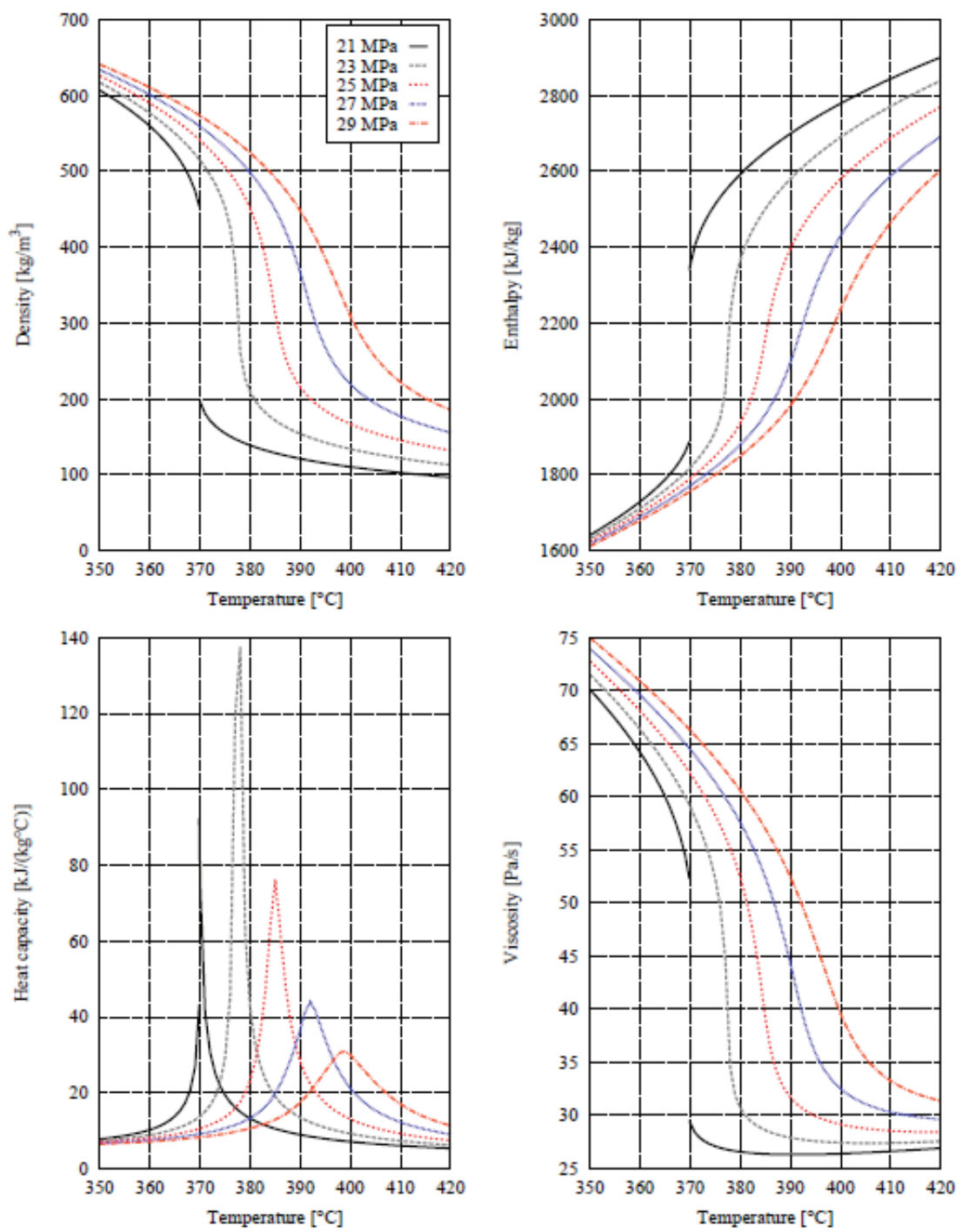

Fig. 1. Thermo-physical properties of water near the pseudo-critical line at various pressures 


\section{Treatment of phase change}

In order to keep the solution structure close to the original two-phase flow model at the supercritical pressure region, the mass transfer rate between the pseudo-liquid and the pseudo-gas is calculated and taken into account in the mass and energy equations of both phases. At subcritical pressures the mass transfer is calculated from the equation

$$
\Gamma=-\frac{q_{\mathrm{i} 1}+q_{\mathrm{ig},}-q_{\mathrm{wi}}}{h_{\mathrm{g}, \mathrm{sat}}-h_{1, \mathrm{sat}}}
$$

In Equation (5) the heat fluxes from liquid to interface and from gas to interface $q_{\mathrm{il}}$ and $q_{\mathrm{ig}}$ are calculated using the interface heat transfer coefficients based on experimental correlations. The term $q_{\mathrm{wi}}$ is the heat flux from wall directly to the interface used for evaporation and condensation. Because at supercritical pressures the mass transfer rate doesn't have any physical meaning it was found to be more practical instead of using the heat fluxes just to force the state of the fluid either to pseudo-liquid or to pseudo-gas. This was done by introducing model for forced mass transfer. In this model the heat flux from wall to interface $q_{\text {wi }}$ was omitted. The forced mass transfer is then calculated as

$$
\Gamma_{\mathrm{F}}= \begin{cases}\frac{\left(1-\alpha^{t-\Delta t}\right) \rho_{1}^{t-\Delta t}}{\Delta t}+\frac{\dot{m}_{i \mathrm{n}, \mathrm{l}}}{\Delta t}, & \text { if } h_{\mathrm{b}}>h_{\mathrm{sat}, \mathrm{l}} \\ \frac{\alpha_{\mathrm{g}}^{t-\Delta t}}{\Delta t}-\frac{\dot{m}_{\mathrm{in}, \mathrm{g}}}{\Delta t}, & \text { if } h_{\mathrm{b}}<h_{\mathrm{sat}, \mathrm{g}} \\ 0, & \text { if } h<h_{\mathrm{b}}<h_{\mathrm{sat}, \mathrm{g}}\end{cases}
$$

For the treatment of the pseudo evaporation/condensation the concept of the pseudo-latentheat is needed to separate the saturation enthalpies of liquid and gas. By using the pseudolatent heat the pseudo-saturation enthalpies of liquid and gas can be expressed as

$$
\begin{aligned}
& h_{\mathrm{sat}, \mathrm{l}}=h_{\mathrm{pc}}-\frac{h_{\mathrm{lg}}}{2} \\
& h_{\mathrm{sat}, \mathrm{g}}=h_{\mathrm{pc}}+\frac{h_{\mathrm{lg}}}{2}
\end{aligned}
$$

In Equations (7) and (8) the pseudo latent heat $h_{\mathrm{gg}}$ is chosen to be small in order to have fast enough transition from pseudo liquid to pseudo gas or vice versa. The use of very small value for pseudo latent heat $(<100 \mathrm{~J} / \mathrm{kg})$ may require small time steps to avoid numerical problems. However, it is also important that the transition does not happen too slowly, because a state with the presence of two separate phases with different temperatures at the same time is not physical.

The calculated mass transfer rate is taken into account in mass, momentum and energy equations of both phases. The pseudo saturation enthalpies are used when the interfacial heat transfer rates are calculated to fulfill the energy balances of the two fluid phases.

The presented model has been widely tested and it works well even for very fast transients (Kurki \& Hänninen, 2010, Kurki 2010). 


\section{Wall heat transfer}

Above the critical pressure the boiling and condensation phenomena cease to exist and only one-phase convection occurs. Due to the pseudo two-phase conditions convection has to be calculated for both the liquid and the gas phase. In the model the heat transfer coefficient is calculated by weighing the pseudo liquid and gas phase coefficients with the gas volumetric fraction, i.e. the supercritical coefficient is calculated as

$$
\mathrm{Nu}_{\mathrm{b}}=\alpha \mathrm{Nu}_{\mathrm{ps}, g}+(1-\alpha) \mathrm{Nu}_{\mathrm{ps}, 1}
$$

The subscript $b$ refers to bulk fluid, and the subscripts ps,g and ps,l to pseudo-gas and pseudo-liquid. In the model most of time void fraction $a$ is either 1 or 0 , i.e. the case where liquid and gas are at different state is temporary. The transition speed from pseudo liquid to pseudo gas depends on the size of the pseudo evaporation heat and on the model to calculate the mass and energy transfer between phases.

Above the critical pressure point the forced-convection heat transfer from wall to both pseudo liquid and pseudo gas is calculated with the correlation of Jackson and Hall (Jackson 2008). The exponent $n$ depends on the ratios of bulk-, wall- and pseudo-critical temperatures. The correlation gives good values for the supercritical pressure heat transfer, but it does not predict the deterioration of heat transfer.

$$
n= \begin{cases}\mathrm{Nu}_{b}=0.0183 \operatorname{Re}_{b}^{0.82} \operatorname{Pr}_{b}^{0.5}\left(\frac{\rho_{w}}{\rho_{b}}\right)^{0.3}\left(\frac{\overline{c_{p}}}{c_{p, b}}\right)^{n} \\ 0.4+0.2\left(\frac{T_{w}}{T_{\mathrm{pc}}}-1\right), & \text { if } T_{b}<T_{w}<T_{\mathrm{pc}} \quad \text { or } 1.2 \mathrm{~T}_{\mathrm{pc}}<T_{b}<T_{w} \\ 0.4+0.2\left(\frac{T_{w}}{T_{\mathrm{pc}}}-1\right)\left(1-5\left(\frac{T_{b}}{T_{\mathrm{pc}}}-1\right)\right), & \text { if } T_{b}<T_{\mathrm{pc}}<T_{w}\end{cases}
$$

The heat capacity $\bar{c}_{p}$ is calculated as an average of values in bulk flow and at wall conditions. In the six-equation model of APROS, also three other forced-convection heat transfer correlations are available as an option. The commonly used subcritical-pressure correlation of Dittus and Boelter gives reasonable values in some situations but in general it exaggerates the heat transfer near the critical point. This is due to extreme high heat capacity value near the critical pressure point (see Figure 1).

Two other forced-convection correlations implemented in APROS are those of Bishop and Watts and Chou (Watts and Chou, 1982). Again, the correlations give generally sensible prediction of the heat transfer coefficient, but only in the absence of the heat transfer deterioration phenomena caused by flow acceleration and buoyancy.

The Watts and Chou correlation uses a buoyancy parameter that tries to take into account the density difference between the fluid at wall temperature and the fluid at the bulk temperature. 
For vertical upward flow, the correlation takes the form

$$
\mathrm{Nu}_{\mathrm{b}}= \begin{cases}\mathrm{Nu}_{\mathrm{var}}(1-3000 \chi)^{0.295}, & \text { if } 10^{-5} \leq \chi \leq 10^{-4} \\ \mathrm{Nu}_{\mathrm{var}}(7000 \chi)^{0.295}, & \text { if } 10^{-4}<\chi\end{cases}
$$

For vertical downward flow the correlation gives the Nusselt number as

$$
\mathrm{Nu}_{\mathrm{b}}=\mathrm{Nu}_{\mathrm{var}}(1+30000 \chi)^{0.295},
$$

meaning that heat transfer is enhanced when the influence of buoyancy is increased. The term $\mathrm{Nu}_{\mathrm{var}}$ takes into account "normal" convection and material property terms

$$
\mathrm{Nu}_{\mathrm{var}}=0.021 \operatorname{Re}_{\mathrm{b}}^{0.8} \operatorname{Pr}^{0.55}\left(\rho_{\mathrm{w}} / \rho_{\mathrm{b}}\right)^{0.35},
$$

and the buoyancy parameter is defined as

$$
\chi=\overline{\mathrm{G}} \mathrm{r}_{\mathrm{b}} /\left(\operatorname{Re}_{\mathrm{b}}^{2.7} \overline{\mathrm{P}}_{\mathrm{b}}^{0.5}\right)
$$

It can be seen that with the buoyancy parameter from $10^{-5}$ to $10^{-4}$ the Nusselt number is decreasing with increasing values of the buoyancy parameter (about $10 \%$ ) and above $10^{-4}$ Nusselt number is increasing.

This correlation takes into account buoyancy-influenced heat transfer but it does not take into account the acceleration-influenced heat transfer impairment.
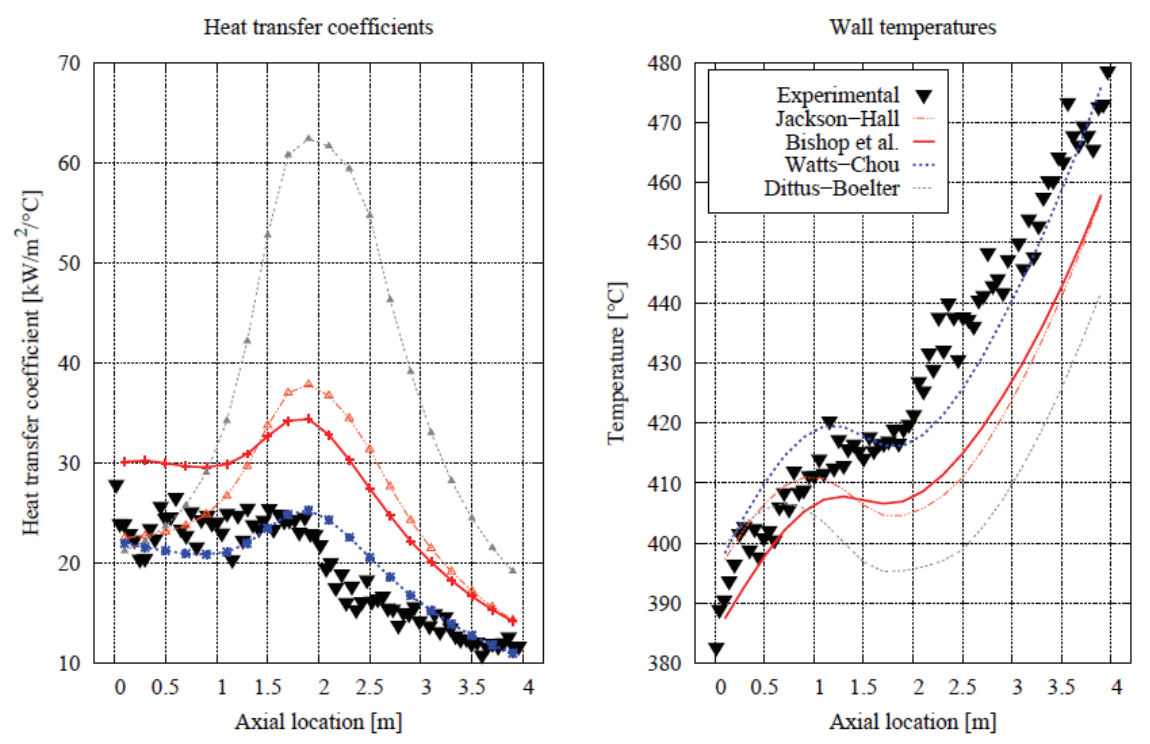

Fig. 2. Heated vertical pipe (IAEA benchmark 1). Effect of different heat transfer correlations, calculated with APROS (Kurki 2010). Experimental data are from (Kirillov et. al., 2005)

APROS includes the four heat transfer correlations which can be used at supercritical pressure flows - Dittus-Boelter, Bishop, Jackson-Hall and Watts-Chou. These correlations 
were used for simulating a test case (Kirillov et. al., 2005), in which the steady-state heat transfer behaviour in a heated vertical pipe was analysed for both upwards and downwards flows (see Figure 2). The length of pipe was $4 \mathrm{~m}$, the inner diameter was $1 \mathrm{~cm}$, pressure at outlet was $24.05 \mathrm{MPa}$, inlet temperature was $352{ }^{\circ} \mathrm{C}$ and mass flux was $0.1178 \mathrm{~kg} / \mathrm{m}^{2} \mathrm{~s}$. In case of upwards flow the weak impairment of heat transfer occurs. It can be seen that the Watts-Chou correlation is able to predict this quite well, while the Dittus-Boelter correlation gives much too high values. The reason for too high values is that the heat capacity used in Prandtl number increase strongly near the pseudo saturation state of $24 \mathrm{MPa}$. For downward flow all of the correlations give sensible values - again Watts-Chou giving the closest values. It should be kept in mind that this is only one example. It has been found that any of available heat transfer correlations cannot predict the heat transfer impairments at all conditions.

\section{Wall friction}

Estimation of two-phase flow wall friction in system codes is generally based on singlephase friction factors, which are then corrected for the presence of two separate phases using special two-phase multipliers. The wall friction factor for single phase flow is often calculated using the Colebrook equation, which takes into account also the roughness of the wall

$$
\frac{1}{\sqrt{f_{\mathrm{col}}}}=1.74-2 \log \left[\frac{1}{\sqrt{f_{\mathrm{col}}}} \frac{18.7}{\mathrm{Re}}+2 \frac{\varepsilon}{D_{H}}\right],
$$

where $\varepsilon$ is the relative roughness of the flow channel wall.

Because at supercritical pressures the variations of the thermophysical properties as a function of temperature may be very rapid, the properties near a heated wall - where the skin friction takes place - may differ considerably from the bulk properties. The friction factors can be corrected to take this into account by multiplying the factor by the ratio of a property calculated at the wall temperature to property calculated at the bulk fluid temperature. One of the friction correlations intended for supercritical pressure is the correlation of Kirillov

$$
f_{\text {kir }}=\left(1.82 \log _{10} \operatorname{Re}_{\mathrm{b}}-1.64\right)^{-2}\left(\frac{\rho_{\mathrm{w}}}{\rho_{\mathrm{b}}}\right)^{0.4}
$$

This correlation has been formed by multiplying the friction factor correlation of Filonenko (the first term) by a correction term based on the ratio of densities calculated at wall and bulk temperatures respectively. However, this correlation is valid only for flows in smooth pipes.

Since no wall friction correlation for supercritical-pressure flows in rough pipes is available in the open literature, a pragmatic approach was taken in APROS to make it possible to estimate the wall friction in such a situation: the friction factor obtained from the Colebrook equation is simply multiplied by the same correction term that was used by Kirillov to extend the applicability of the Filonenko correlation, thus the friction factor may be calculated as 


$$
f=f_{\mathrm{col}}\left(\frac{\rho_{\mathrm{w}}}{\rho_{\mathrm{b}}}\right)^{0.4}
$$

It is important to notice that this correlation is not based on any real data, and as such it must not be used for any real-life purposes before it has been carefully validated against experimental results. Thus, this form of the correlation serves only for preliminary estimation of the effect of friction and is mainly intended for reference purposes (Kurki 2010).

\section{Flow instabilities in heated channels}

In simulating flows and heat transfer at the supercritical pressure region the possibility of appearance of flow instabilities should be taken into account. Due to the rapid changes of density and viscosity with changing temperature near the pseudocritical line, different types of flow instabilities may occur. These instabilities are analogous to those related to boiling in vertical pipes, and may be of the Ledinegg or the density-wave-oscillation (DWO) type. Useful dimensionless parameters for defining the condition for stable or instable flows in heated pipes are the sub-pseudo-critical and trans-pseudo-critical numbers proposed in (Ambrosini \& Sharabi 2006 and 2008).

The sub-pseudo-critical number describes the sub-cooling at the inlet of the heated pipe section, and is calculated as

$$
\mathrm{N}_{\mathrm{spc}}=\frac{\beta_{\mathrm{pc}}}{c_{p, \mathrm{pc}}}\left(h_{\mathrm{pc}}-h_{\mathrm{in}}\right),
$$

while the trans-pseudo-critical number represents the proportion of heating power to mass flux, and is defined as

$$
\mathrm{N}_{\mathrm{tpc}}=\frac{P}{\dot{m}_{\mathrm{in}}} \frac{\beta_{\mathrm{pc}}}{c_{p, \mathrm{pc}}} .
$$

With these two parameters the threshold for instabilities and the instable and stable flow areas can be estimated.

In the reference (Ambrosini \& Sharabi, 2008) the stability boundaries of one particular geometry as function of $\mathrm{N}_{\mathrm{spc}}$ and $\mathrm{N}_{\text {tpc }}$ calculated with RELAP5 are shown. The calculated values in the charts have been obtained by simulating the case where the flow under supercritical pressure flows through a vertical uniformly heated circular pipe. At the inlet, the constant singular pressure loss coefficient of $K_{i n}=10.5$ and at the outlet the coefficients of $\mathrm{K}_{\text {out }}=0.0$ and 3.0 were applied. The pressure at inlet and at outlet is kept constant, but the heating rate is gradually increased, which results in a slowly increasing trans-pseudo-critical number. The calculation was repeated with different sub-pseudo-critical numbers corresponding to different inlet conditions. With a certain trans-pseudo-critical number, the flow changes from stable to oscillating or experiences the flow excursion. In the charts the instability threshold is presented when the amplifying parameter $\mathrm{Zr}$ has the value zero. The instability values above the position, where the second derivative changes strongly, represent the Ledinegg instabilities ( $\mathrm{N}_{\mathrm{spc}}$ about 3 ). The values below $\mathrm{N}_{\mathrm{spc}}$ about 3 stand for 
density instabilities. As an example, two calculation results obtained with APROS have been shown in Figures 3 and 4 . In Figure 3 the typical behavior of a DWO-type instability is shown. The result representing the Ledinegg instability is presented in Figure 4.

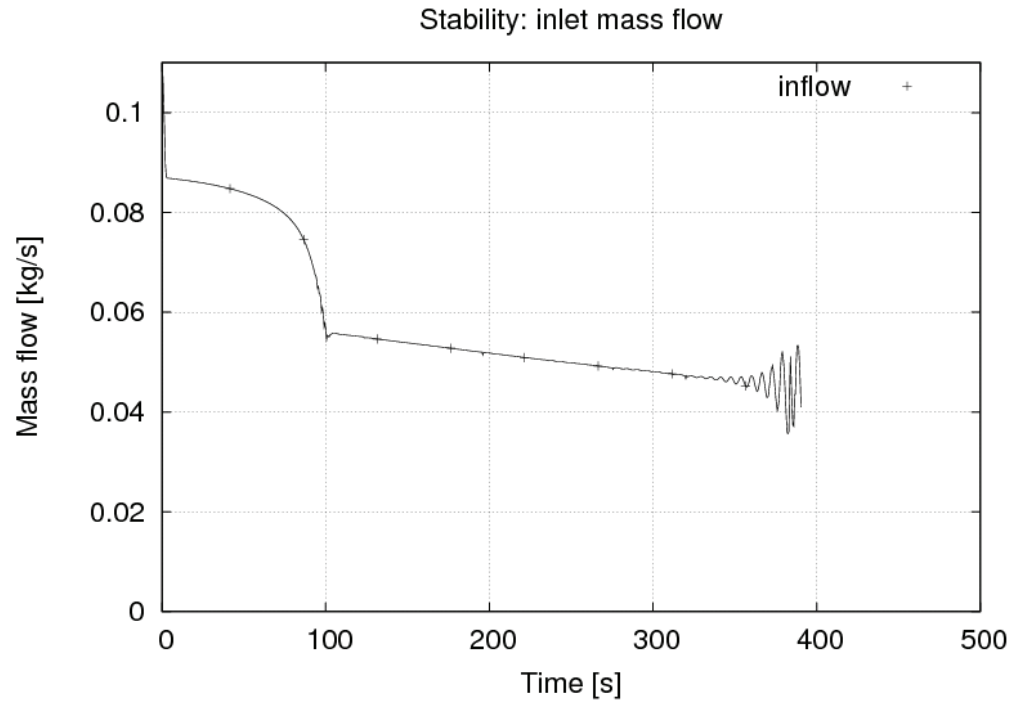

Fig. 3. Example of oscillating type (density) instability (IAEA benchmark 2, $\mathrm{N}_{\mathrm{spc}}=2.0, \mathrm{~K}_{\mathrm{in}}=20$, $\left.\mathrm{K}_{\text {out }}=20\right)$, Calculated with APROS

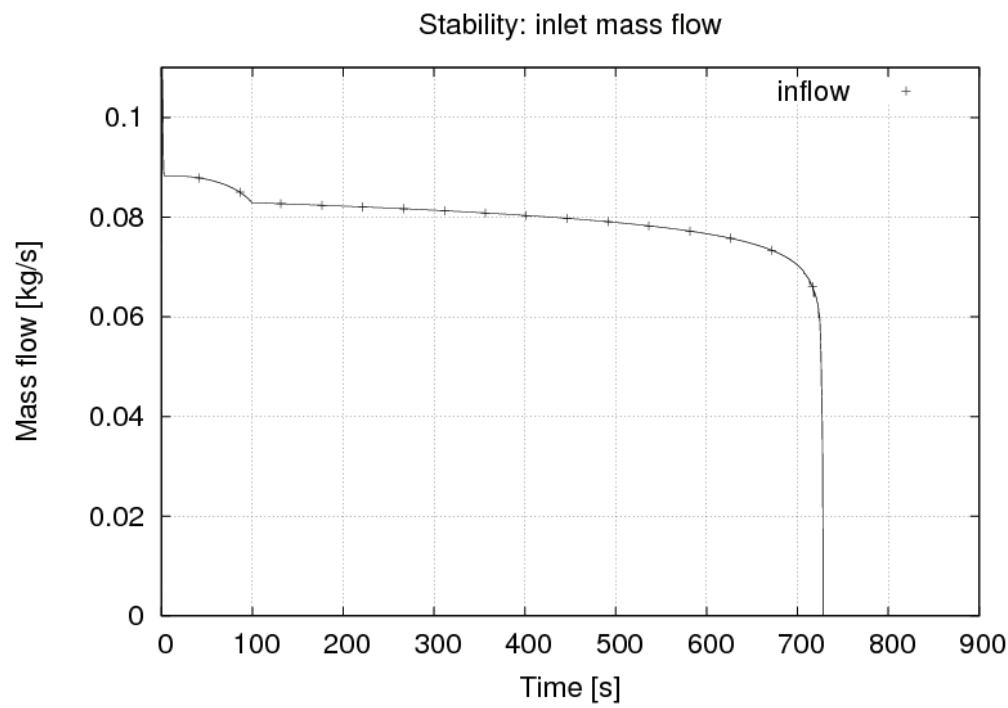

Fig. 4. Example of excursion type (Ledinegg) instability $\left(\mathrm{N}_{\mathrm{spc}}=3.0, \mathrm{~K}_{\mathrm{in}}=20, \mathrm{~K}_{\mathrm{out}}=20\right.$ ), Calculated with APROS 


\section{Simulation application - European concept of supercritical reactor (HPLWR)}

In an European collaboration project, the concept of a new reactor working under the supercritical pressure has been developed (Schulenberg \& al. 2008). This concept, called High Performance Light Water Reactor (HPLWR), has a three-pass core (Fischer \& al, 2009), which was introduced to the design for preventing the formation of hot spots in the reactor core. The coolant flows through the core three times in the radially separated evaporator, super-heater 1 and super-heater 2 sections. Half of the feed water coming into the reactor pressure vessel is directed upwards to the upper plenum in order to provide neutron moderation for the reactor core and the other half flows to the downcomer, from where it continues to the core coolant channels. Also for the moderator, a three-stage flow scheme is applied.

For some preliminary accident analyses of the HPLWR safety concept, the APROS system code was used (Kurki \& Hänninen, 2010). The intention of calculation was on the other hand make a typical large break LOCA analysis and also to verify the feasibility of the tentative protection system. The accident which was analysed was a guillotine break of one of the four steam lines. The accident was initiated by a $2 \times 100 \%$ break between the pressure vessel outlet and the main steam line isolation valve. Decreasing pressure at the pressure vessel outlet ( $p_{\text {out }}<225$ bar) initiates the reactor scram sequence and closure of the main steam line isolation valves (MSIV). The time taken for fully closing the isolation valves is assumed to be 3.5 seconds. After the MSIV has been shut, the effective break size is $1 \times 100 \%$.

The calculation results (see Figure 5) suggested that with the assumed protection strategy and with the used safety injection capacity the reactor core of the HPLWR can be kept sufficiently cooled-down in the case of a large break LOCA caused by rupture of one of the four main steam lines, using the designed safety systems.
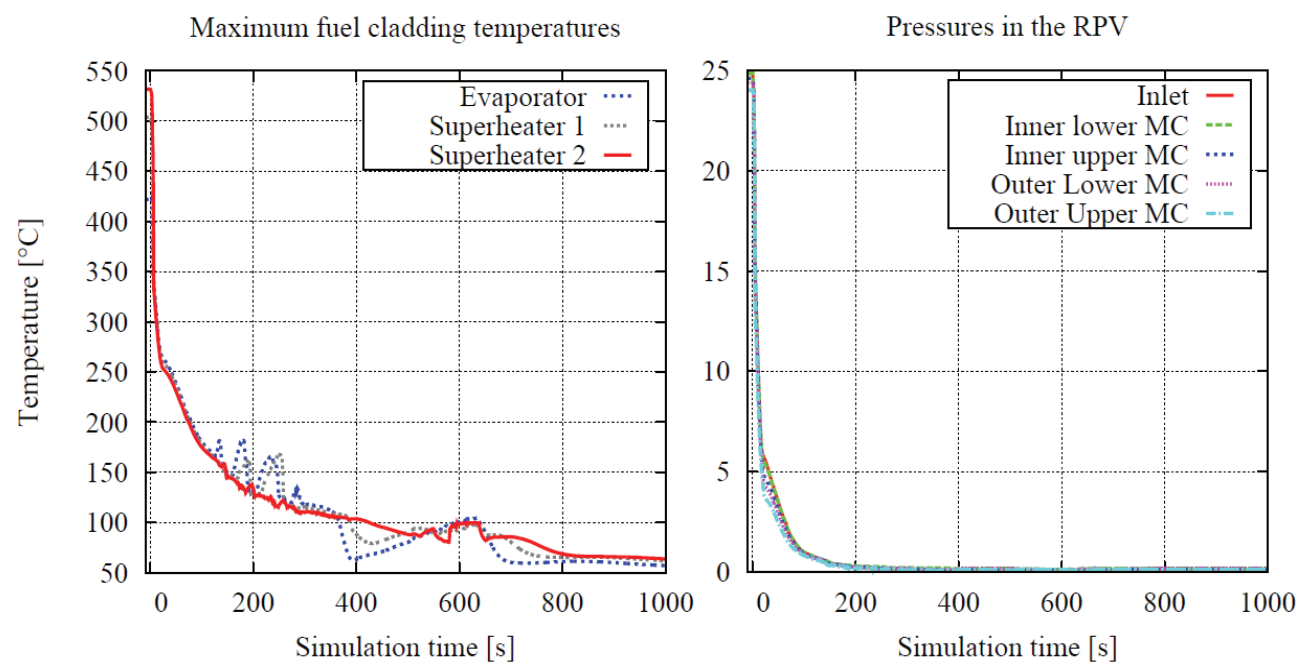

Fig. 5. Results from the HPLWR LB-LOCA simulations: maximum flue cladding temperatures (left) and pressures in different parts of the pressure vessel (right) 


\section{Conclusion}

With modifications described in this paper, the six-equation flow model of the system code APROS has been updated to work near and above the critical point. By using the concept of pseudo saturation enthalpy the two-phase structure can be maintained. The developed model for the forced mass transfer enables the numerically stable and fast transition from pseudo-liquid to pseudo-gas and vice versa. The model includes a selection of friction and heat transfer correlations that the user can choose to be used at supercritical pressures. One deficiency is that at the moment there is not any heat transfer correlation which is able to calculate the impairment of the heat transfer reliably. In simulating the supercritical flows in heated pipes the instabilities due to large density changes is possible. In making simulations with system codes or other simulation tools it is necessary to know the limits of these instabilities. As an example of the HPLWR LOCA simulation proves the developed model can be used for the safety analysis of supercritical-water-cooled reactors.

\section{Nomenclature}

A area, flow area $\left(\mathrm{m}^{2}\right)$

$\beta \quad$ volumetric coefficient of expansion

$f \quad$ friction factor

$F \quad$ friction $\left(\mathrm{N} / \mathrm{m}^{3}\right)$

$g$ acceleration of gravitation $\left(\mathrm{m} / \mathrm{s}^{2}\right)$

$h \quad$ enthalpy $(\mathrm{J} / \mathrm{kg})$

$\dot{m} \quad$ mass flow $(\mathrm{kg} / \mathrm{s})$

$\mathrm{Nu} \quad$ Nusselt number

$p \quad$ pressure $(\mathrm{Pa})$

Pr Prandtl number

$q \quad$ heat flow/volume

Re Reynolds number

$\mathrm{T}$ temperature $\left({ }^{\circ} \mathrm{C}\right.$ or $\left.\mathrm{K}\right)$

$t$ time (s)

$u \quad$ velocity $(\mathrm{m} / \mathrm{s})$

$V \quad$ volume $\left(\mathrm{m}^{3}\right)$

$x \quad$ mass fraction

$\alpha \quad$ gas volume fraction

$\rho$ density $\left(\mathrm{kg} / \mathrm{m}^{3}\right)$

$\Gamma \quad$ mass transfer $\left(\mathrm{kg} / \mathrm{s} \mathrm{m}^{3}\right)$

$\Delta t \quad$ time step (s)

$T$ temperature (C)

$\Delta z \quad$ mesh spacing in flow direction (m)

\section{Subscripts}

$b \quad$ bulk

col Colebrook

$g$ gas

$i \quad$ interface

$k \quad$ phase $\mathrm{k}$ (either gas or liquid) 


$\begin{array}{ll}\text { kir } & \text { Kirillov } \\ l & \text { liquid } \\ l g & \text { evaporation, liquid to gas } \\ p & \text { constant pressure } \\ p c & \text { pseudo critical } \\ \text { sat } & \text { saturation } \\ \text { w } & \text { wall }\end{array}$

\section{Superscript}

$n \quad$ new value

\section{References}

Ambrosini, W., and Sharabi, M., 2006, Dimensionless Parameters in Stability Analysis of Heated Channels with Fluids at Supercritical Pressures, Proceedings of ICONE 14, 14th International Conference on Nuclear Engineering, July 17-20, 2006, Miami, Florida USA.

Ambrosini, W., and Sharabi, M., 2008, Dimensionless Parameters in Stability Analysis of Heated Channels with Fluids at Supercritical Pressures, Nuclear Engineering and Design 238 (2008) 1917-1929.

Bishop, A. A., Sandberg, R.O., Tong, L.S., Forced convection heat transfer to water near critical temperatures and supercritical pressures, Report WCAP-5449, Westinghouse Electrical Corporation, Atomic Power Division (1965).

Fischer, K., Schulenberg, T., Laurien, E., Design of a supercritical water-cooled reactor with a three pass core arrangement. Nuclear Engineering and Design, 239 (2009), pp. 800812.

Hänninen, M. \& Kurki, J. Simulation of flows at supercritical pressures with a two-fluid code. NUTHOS-7: The 7th International Topical Meeting on Nuclear Reactor Thermal Hydraulics, Operation and Safety, Seoul, Korea, October 5-9, 2008

Hänninen, M. and Ylijoki, J. The on-dimensional separate two-phase flow model of APROS. Espoo 2008. VTT Tiedotteita - Research Notes.

Jackson, J.D., "A Semi-empirical model of turbulent convective heat transfer to fluids at supercritical pressure, Proceedings of the 16th International Conference on Nuclear Engineering, ICONE16 May 11-15, 2008, Orlando, Florida.

P. Kirillov and V. Grabezhnaya, Heat transfer at supercritical pressures and the onset of deterioration, in Proceedings of ICONE14 (Jul 2006)

P. Kirillov, R. Pomet'ko, A. Smirnov, V. Grabezhnaia, I. Pioro, R. Duffey and H. Khartabil, Experimental Study on Heat Transfer to Supercritical Water Flowing in 1- and 4-mLong Vertical Tubes, in Proceedings of GLOBAL 2005 (2005)

Kurki, J., Simulation of thermal hydraulics at supercritical pressures with APROS, Thesis for the degree of Master of Science in Technology, Helsinki University of Technology, Department of Engineering Physics and Mathematics, 2008.

Kurki, J. and Hänninen, M., Simulation of flows at supercritical pressures with a two-fluid code, in Proceedings of the 8th International Topical Meeting on Nuclear Reactor Thermal Hydraulics, Operation and Safety (NUTHOS-8) (October 2010

Kurki, J. and Seppälä M., Thermal Hydraulic Transient Analysis of the High Performance Light Water Reactor Using APROS and SMABRE, 20th International Conference 
on Structural Mechanics in Reactor Technology (SMiRT 20), Espoo, Finland, August 9 -14, 2009, SMiRT 20 - Division X, Paper 3164

J. Kurki and M. Hänninen, Simulation of a Large-Break Loss of Coolant Accident in the High Performance Light Water Reactor, in Proceedings of IAEA Technical Meeting on 'Heat Transfer, Thermal-Hydraulics and System Design for Supercritical Water Cooled Reactors' (July 2010)

Kurki, J., Computational modeling of a supercritical-Water-Cooled Reactor, Thesis for the degree of Licentiate of Science in Technology, Aalto University, School of Science and Technology, 2010.

Schulenberg, T., Starflinger, J., Heinecke, J., Three pass core design proposal for a high performance light water reactor. Progress in Nuclear Energy, 50 (2008), pp. 526-531.

Watts, M. J., and Chou, C. T., Mixed Convection Heat Transfer to Supercritical Pressure Water, Proceedings of the 7th International Heat Transfer Conference, vol. 3, 495 500 (1982) 


\title{
Non-Linear Design Evaluation of Class 1-3 Nuclear Power Piping
}

\author{
Lingfu Zeng, Lennart G. Jansson and Lars Dahlström \\ $\AA$ A-Industry $A B$ \\ Box 1551, SE-401 51 Göteborg, \\ Sweden
}

\section{Introduction}

A nuclear piping system which is found to be disqualified, i.e. overstressed, in design evaluation using linear analysis software in accordance with ASME Boiler \& Pressure Vessel Code, Section III (ASME, 2009a), denoted as ASME III below for convenience, can still be qualified if further design requirements can be satisfied in refined nonlinear finite element analyses in which material plasticity and other non-linear conditions are taken into account. For clarity, a design evaluation using such linear analysis software will throughout this chapter be called a linear design evaluation, and a design evaluation involving a non-linear finite element analysis a non-linear design evaluation.

The linear design evaluation according to ASME III is purely based on stress limits. Stresses in piping components are first divided into membrane, bending and localized stresses for formulation consistency with beam and/or shell structures. Thereafter, stresses are further categorized into primary, secondary and peak stresses. The primary stresses are the "not self-limiting" part of responses typically resulted from external forces such as dead-weight, internal pressure, earthquake and so on, and they are important to avoid catastrophic failure and to control plastic deformation. The secondary stresses refer to the "self-limiting" part of responses resulted typically from thermal effects and gross structural/material discontinuities, and they are responsible for eventually progressive/incremental deformation. The peak stresses are the combined "peak" responses which are used to control fatigue failure. In ASME III, design criteria are defined in terms of stress intensity or principal stresses. For Class 1 piping systems, the criteria are defined by the stress intensity which is the largest absolute value of the principal stress difference, or equivalently twice of the maximum shear stress, and for Class 2 and 3 piping systems by the largest absolute value of the principal stresses. In connection with the design-by-analysis approach, the linear design evaluation is performed through comparing stress intensities of above-mentioned stress categories with their allowable limits. Among software commercially available for performing such a linear design evaluation, PIPESTRESS from DST Computer Services S.A. (DST, 2005) is widely used in Sweden.

Furthermore, the linear design evaluation is conducted for each of the following load sets: Design Condition and 4 so-called Service Limits of Level $A, B, C$ and $D$. For different load sets, different design criteria and requirements are used. Through defining various loads 
into different load sets and using different design criteria and requirements, the safety degree consideration and the occurrence probability of a given load can be introduced in the design evaluation.

In accordance with ASME III, non-linear design evaluation is an alternative to the linear design evaluation. Depending on which stress intensity limit is violated in the linear design evaluation, there are two types of non-linear analysis required in ASME III for the alternative non-linear design evaluations: (1) collapse-load analysis and (2) non-linear transient analysis. For clarity, such alternative design criteria and requirements which are specified in connection with such non-linear analyses are termed hereby as the non-linear design criteria and requirements. Such non-linear finite element analyses can generally effectively be conducted using general-purpose finite element software, such as ANSYS (ANSYS, 2010) and most other well recognized software.

This Chapter is devoted to describe the general procedure for the alternative non-linear piping design and to clarify those relevant non-linear design criteria and requirements. Our emphasis will be placed on the later task as unclear and inconsistent issues have been observed in ASME III when non-linear design criteria and requirements applied. In recent years, quite many non-linear analyses and design evaluations have been conducted in Sweden for several power uprate projects. Unfortunately, most of such work has always ended with, or can never be ended without, long discussions on such unclear and inconsistent issues.

The Chapter is organized as follows: In Section 2, an overview on loading conditions is given. In Section 3, we review the linear design evaluation and discuss the non-linear design evaluation for Class 1 piping systems. In Section 4, the review and discussion are continued for Class 2 and 3 piping. In Section 5, we briefly address the computational procedures for collapse-load analysis and, in Section 6, we discuss the computational procedures for nonlinear transient analysis. Finally, in Section 7 concluding remarks are given. We note that the discussion given in this Chapter is mainly based on our experiences on the application of ASME III under Nordic conditions, see e.g. Zeng (2007), Zeng \& Jansson (2008), Zeng et al. $(2009,2010)$.

As this chapter covers a large amount of design rules and requirements of ASME III, an attempt has been made to keep the presentation brief and concise, yet still sufficiently clear. Unless otherwise stated, notations will be kept to be identical to those used in ASME III, equations specified in ASME III will not be repeated here unless necessary, and fundamental design requirements e.g. Pressure Design etc., will not be discussed here. In particular, the description of the linear design evaluation will be kept brief whenever possible and, for a more detailed description, we refer to ASME (2009), Slagis \& Kitz (1986), Slagis (1987) and references therein.

\section{Load conditions}

The design evaluation rules in ASME III are for Class 1, 2 and 3 piping specified in terms of 5 loading conditions: Design Condition, and Service limits of Level A, B, C and D.

Under each loading condition, loads are combined to one or several load set(s) according to Design Specifications. The rules for load-combinations are defined in accordance with probabilities in which corresponding loads (events) should occur and consequences that may result in. Thus, a given load set defines the following: 
1. Loads and their combinations to be considered in piping analysis.

2. Stress intensity limits to be used in the subsequent design evaluation.

In Tab. 1 we show an example of how these load sets are specified in Sweden. The design evaluation must be conducted in accordance with this table and the piping design is not qualified unless all evaluation rules specified for each load set are met.

We note that in Tab. 1 notations are of self-explaining, e.g. PD for Design Pressure and SSE for Safe-Shutdown Earthquake etc. Rather than explaining how load-combinations are defined in Tab. 1, which is not our purpose, we should observe the followings from this table:

1. Loads given under Design Condition are not only static loads of Design Pressure (PD) and Dead Weight (DW), but also dynamic loads (GV/SRV1) which represents here those generated by opening or closing one safety valve.

2. Loads in Service limit Level A include static loads (PO+DW) and dynamic loads GV/SRV1, where PO denotes the operating temperature. We note that GV/SRV1 are generally not included according to ASME III, and they appear here due to additional requirements specified in Swedish design specifications.

3. Loads in Service limit Level B include static loads $(\mathrm{PO}+\mathrm{DW})$, time-dependent loads generated by opening or closing of seven safety valves (GV/SRV7).

4. Loads in Service limits of Level C and D include static loads (PO+DW), dynamic loads generated by e.g. opening or closing of several safety valves, and Safe-Shutdown Earthquake (SSE) and so forth.

Tab. 1 is only an example for our discussion purpose. In practice, more load cases and combinations need to be considered, such as Water-Hammer loads (WH), local vibration due to safety relief of valves, Local vibration due to chugging, Pool swell drag due to internal pipe break, Pool swell impact due to internal pipe break and several others.

\begin{tabular}{|l|l|l|l|}
\hline Load-combinations & $\begin{array}{l}\text { Design and/or } \\
\text { Service limit Level }\end{array}$ & Pressure & Temp. \\
\hline PD + DW & Design & PD & TD \\
\hline PD + DW + GV/SRV1* & Design & PD & TD \\
\hline PO + DW + GV/SRV1* & Level A & PO & TO \\
\hline PO + DW + GV/SRV7 (E-3) & Level B & PO & TO \\
\hline PO + DW + SRSS(GV/SRV7(E-2), WH/VO1) & Level C & PO & TO \\
\hline PO + DW + SRSS(GV/SRV7(E-3), GV/SSE) & Level D & PO & TO \\
\hline
\end{tabular}

Table 1. Load-combinations and their evaluation specifications

It should be noticed that time-dependent loads can be either given in form of response spectra, which are the case when GV/SSE and GV/SRV or other global vibration (GV) related events considered, or in form of time-dependent "nodal forces" $F(t)$ which are in most cases generated in separate fluid dynamic analyses.

Time-dependent loads $F(t)$ can be reversing, non-reversing or non-reversing followed by reversing, see NB-3620, NC-3620 (ASME, 2009a). In Fig.1 we show an example of nonreversing followed by reversing $F(t)$ caused typically by an initial water slug followed by 
reflected pressure pulses. As we will see later, some design rules, in particular, those nonlinear ones are given in terms of the types of dynamic loads. When dealing with dynamic loads, it is therefore important to distinguish reversing and non-reversing types.

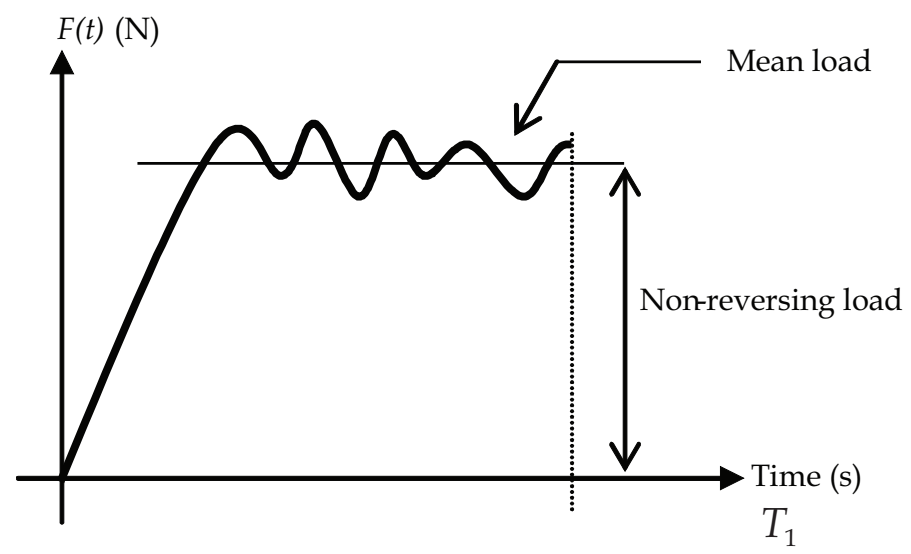

Fig. 1. Dynamic loading of a non-reversing type followed by a reversing type

\section{Class 1 piping}

The linear design rules for Class 1 piping are given in NB-3600 for general rules and in NB3650 - NB-3656 for specific rules. When the linear design rules unsatisfied, in other words, the piping design found to be disqualified, the piping can still be qualified if alternative nonlinear design requirements specified generally in NB-3200 Design by Analysis, where material plasticity are treated in NB-3228, can be met. In this section, we follow the rules specified in NB-3600 for each specific load set to clarify these non-linear design requirements. In ASME III, different design requirements are, in general, specified in terms of two types of loads: (1) Loads including non-reversing dynamic loads or non-reversing followed by reversing dynamic loads; (2) Loads including reversing dynamic loads. The definitions for reversing and non-reversing dynamic loads are given in NB-3622 and repeated below:

Reversing dynamic loads are those loads which cycle about a mean value and include building filtered loads, earthquake, and reflected waves in piping due to flow transients resulting from sudden opening/closure of valves. A reversing load shall be treated as nonreversing when the following condition is met: The number of reversing dynamic cycles, excluding earthquake, exceeds 20.

Non-reversing dynamic loads are those which do not cycle around a mean value, and include initial thrust forces due to sudden opening/closure of valves and water-hammer resulting from entrapped water in two-phase flow.

\subsection{Design condition}

The linear design evaluation for this load set is to evaluate Eq.(9) given in NB-3652 to ensure the primary (primary membrane plus primary bending) stress intensity is within its limit $1.5 S_{\mathrm{m}}$, where $S_{\mathrm{m}}$ is the allowable design stress intensity value. According to NB-3228.1 or NB-3228.3, the non-linear design requirements can be formulated as follows: If Eq.(9) 
unsatisfied, a non-linear analysis can be made to predict the collapse-load and the design can still be considered to be qualified if the applied loads do not exceed 2/3 of the collapseload. The collapse-load may be predicted either by a Limit Analysis procedure specified in NB-3228.1 or a Plastic Analysis procedure specified in NB-3228.3.

There is a fundamental difference between these two procedures. While the Limit Analysis procedure aims at predicting the lower bound of the collapse-load, the Plastic Analysis procedure implies a prediction of the whole load-displacement history until the structure reaches, or passes through, its collapse point. The prediction of the collapse-load will be elaborated in Sect. 5 .

In addition to this fundamental difference, NB-3228 requires the following:

For the Limit Analysis, the material is assumed to be perfectly elastic-plastic, and the yield stress is set to $1.5 S_{\mathrm{m}}$. The yield stress can be reduced for some materials, see NB-3228.1. A von Mises yield criterion is used. The lower bound of the collapse-load can be computed incrementally or by other available procedures. Here, the historic behavior in the piping during the loading process, such as plastic strains, is of no interest.

The Plastic Analysis requires that the true material stress-strain relation, including strain hardening behavior, should be used. A von Mises yield condition is still assumed and the initial yield stress must be set to the true yield stress $S_{\mathrm{y}}$. The collapse-load can only be computed by an incremental procedure and it can only be determined when (almost) the whole historic behavior in the piping during the whole loading process is computed. Moreover, the collapse-load in this context is a load-level that is determined using a specific procedure given in NB-3213.25, not the load-level corresponding to the collapse point predicted numerically, see Section 5 .

The Limit Analysis is simpler but predicts, however, the lower bound of the collapse-load. It implies generally an application of a stronger evaluation requirement. Nevertheless, it is reasonable to use the Limit Analysis as the first choice when Eq.(9) unsatisfied.

\subsection{Level A}

In the linear design evaluation for all load sets for which Service limit Level A is designated, two types of requirements are to be satisfied: (a) fatigue requirements and (b) thermal ratchet requirements, see NB-3653.

\subsubsection{Fatigue evaluation}

The fatigue requirements are specified in NB-3653.1 - 3653.6. In principle, the following two conditions are verified:

1. Primary plus secondary stress intensity range.

The evaluation is done by using Eq.(10), NB-3653.1, to ensure the stress intensity range $S_{\mathrm{n}} \leq 3 S_{\mathrm{m}}$. The evaluation must be made for all load sets in Level A.

2. Peak stress intensity range.

The evaluation is done by using Eq.(11), NB-3653.2, to determine a so-called alternating stress intensity $S_{\text {alt }}$ (NB-3653.3), which is in turn used to find the allowable number of load cycles $N$ in design fatigue curves (NB-3653.4). Thereafter, a procedure defined in NB-3222.4(e)(5) is applied to estimate the cumulate damage (NB-3653.5). The design is qualified if we find a so-called cumulative usage factor $U \leq 1.0$. This evaluation must be made for all load sets in Level A.

Remark: These fatigue requirements (1) and (2) must also be verified for all load sets which are designated in Service limit Level B, see Section 3.3. When computing the cumulative 
usage factor $U$, all load-sets in Level $A$ and all load sets in Level $B$ must together be taken into account.

Now we shall clarify what we can do if the fatigue requirements (1) and (2) cannot be fulfilled. NB-3653.6 states that if Eq.(10) unsatisfied, one may apply a so-called simplified elastic-plastic discontinuity analysis to evaluate Eqs. (12) and (13), and the cumulative damage factor using a slightly modified procedure, NB-3653.6 (a), (b) and (c). The design is qualified if Eqs.(12) and (13) satisfied, and $U \leq 1.0$.

At this point, one may ask: What can we do if Eq.(10) satisfied, but the cumulative damage factor in Condition (2) found to be $U>1.0$ ? ASME NB is unclear on this point. One may realize that, in the simplified elastic-plastic discontinuity analysis, the alternating stress intensity is increased by a factor $K_{\mathrm{e}} \geq 1.0$ through Eq.(14), which in turn reduces the limit of load-cycles, and consequently increases the cumulative damage factor $U$. In such cases, the simplified elastic-plastic discontinuity analysis will not help in one's attempt to further verify the piping design.

NB-3653.1(b) states that, as an alternative to the simplified elastic-plastic discontinuity analysis in NB-3653.6, one may apply a Simplified elastic-plastic analysis specified in NB3228.5. When discussing this issue, we must remark the following: NB-3600 provides design rules/criteria for only piping. Whereas NB-3200 provides design rules/criteria which are more general and detailed and applicable for all nuclear facility components including piping. In other words, NB-3600 states simplified methods of NB-3200 for performing design-by-analysis for piping. Hence, a piping component which fails to meet conditions in NB-3600 can still be qualified if it meet conditions given in NB-3200. As far as piping concerned, the design rules and requirements given in NB-3200 and NB-3600 should be the same.

We look now back to Eq.(10). Recall that Eq.(10) ensures the primary plus secondary stress intensity range being within its limit $3 S_{\mathrm{m}}$. By examining NB-3220 we find, however, that none of rules given in NB-3228 seems to be directly applicable for doing a further evaluation when $U>1$ found in a simplified elastic-plastic analysis. Furthermore, that NB-3200 does not state any further design requirement if the peak stress intensity range leads to a cumulative usage factor $U>1$.

Now, a question arises: Can we apply non-linear analyses to do a further design assessment when Eq.(10), or Eqs.(12) and (13), unsatisfied and/or the cumulative usage factor found to be $U>1$ ? In Section 3.2.3, we shall attempt to answer this question.

\subsubsection{Thermal stress ratchet evaluation}

The thermal stress ratchet evaluation is given in NB-3653.7 which ensures the range of temperature changes, $\Delta \mathrm{T}_{1}$ range, is within its limit. NB-3653.7 does not state any further assessment rule if the range of temperature changes overshoots its limit. However, in NB3228.4 Shakedown Analysis, it is stated that a refined non-linear analysis, which will be reviewed and discussed in detail in the next Section, can be used to further check if the piping components can still be qualified.

\subsubsection{Non-linear design evaluation}

In NB-3228.4 Shakedown Analysis, both Thermal Stress Ratchet in Shell (NB-3222.5) and Progressive Distortion of Non-integral Connections (NB-3227.3) are discussed. In NB3228.4(b), it is stated that the design can be considered to be acceptable provided that the following two conditions satisfied: 
1. The maximum accumulated local strain at any point, as a result of cyclic operation to which plastic analysis applied, does not exceed $5 \%$.

2. The deformations which occur are within specific limits.

These two conditions will, for convenience in the later discussion, be termed as the $5 \%$ strain limit rule.

The 5\% strain limit rule is according to NB-3228.4(b) a design requirement which replaces the following specific requirements: (1) NB-3221.2 Local membrane stress intensity being less than $1.5 S_{\mathrm{m}} ;(2)$ NB-3222.2 Primary plus secondary stress intensity range being less than $3 S_{\mathrm{m}}$ i.e. Eq.(10) in NB-3653; (3) NB-3222.5 Thermal stress ratchet, and (4) NB-3227.3 Progressive distortion (deformation) control. In other words, this rule sets a limit of progressive deformation in a shakedown process that may eventually take place. We note that this rule applies for both general piping components and non-integral connections (screwed on caps, screwed in plugs, closures etc).

By thermal stress ratchet it is meant in NB-3222.5 an action, more exactly speaking, a response, in that deformation caused during thermal cyclic loading increases by a nearly equal amount in each cycle. The danger does not lie in the response (deformation) caused in any particular load cycle, but the accumulated amount irreversible (plastic part) response, which may lead to uncontrollable progressive distortion. This may explain why ASME III limits the temperature range $\Delta \mathrm{T}_{1}$ range in the linear design evaluation, but imposes the $5 \%$ strain limit rule when plasticity considered. In all load sets of Service limit Level A, thermal transients (TT) are of main concern. This implies that a shakedown process is irremissible and the 5\% strain limit rule becomes the right choice.

Now, we consider again the fatigue control or evaluation. Does this $5 \%$ strain limit rule cover also the need for fatigue control? Generally speaking, it does not! Damage due to fatigue is a totally different damage phenomenon than that caused by material (plastic) yielding. While the former is mostly dominated by brittle failure in form of micro-fracture and cracking, the later is entirely a ductile failure process in which the dislocation of material crystalline grains is dominating. These two damage mechanisms must be dealt with separately.

To answer how a Class 1 piping under Service limit of Level A should be verified through a non-linear analysis when the linear design evaluation found unsatisfied, the author suggests the following:

1. If the thermal stress ratchet condition unsatisfied, the $5 \%$ strain limit rule can always be applied.

2. If Eq.(10) unsatisfied, the simplified elastic-plastic discontinuity analysis should be the first choice for further evaluation.

3. If Eqs.(12) and (13) unsatisfied, and $U>1$ (evaluated by the procedure given in NB3653.6), the 5\% strain limit rule will be applied first. If this rule unsatisfied, the design cannot be qualified (or must at least be further questioned)! If satisfied, we shall first notify the owner of the nuclear power plant. If the owner requests a further evaluation, a refined approach for calculating the cumulative factor $U$, which is based on the numerical results from non-linear analyses, should be suggested to the contractors (and the owner of the nuclear power). This should be handled on a base of individual cases. If such an approach agreed, the evaluation goes further. Otherwise, the design is declared to be disqualified.

One may argue that the simplified elastic-plastic analysis cannot help if $U>1$ predicted in Step (2) above. The point is, when the simplified elastic-plastic analysis requested in PIPESTRESS for fatigue evaluation, Eqs.(12), (13) and (14) will be evaluated together and, at 
the same time, a updated cumulative factor $U$ will be reported. We remind that, as discussed earlier in Section 3.2.1, if Eq.(10) satisfied but $U>1$, this simplified elastic-plastic analysis cannot alter the result $U>1$.

Furthermore, one may think that it may be possible that, one obtains the following results from a linear analysis using e.g. PIPESTRESS: Eqs.(12) and (13) unsatisfied, but $U \leq 1$. This situation should actually not happen as, according to NB-3653.6, Eqs.(12) and (13) should first be satisfied before computing $U$.

\subsection{Level B}

The linear design evaluation for all load sets for which Service limit Level B is designated, is the same as that for Service limit Level A, see NB-3654. The evaluation requirements are basically given in terms of loads including non-reversing and reversing load types. We notice that the formulation in NB-3654 is unclear with regard to fatigue requirements. More specifically, the first paragraph in NB-3654 contradicts with NB-3654.2, stating whether all load sets in Level A and B, or all load sets in Level A and (only) reversing loads in Level B, should all together be considered when computing the cumulative damage factor in fatigue evaluation. We agree the following:

a. To satisfy Eq.(9) in NB-3652 for non-reversing loads, or reversing loads combined with non-reversing loads (NB-3654.2(a)).

b. To satisfy the fatigue requirements specified in NB-3653.1 through NB-3653.6 for both reversing and non-reversing loads (NB-3654.2(b)).

c. To satisfy the thermal ratchet requirement given in NB-3653.7 for all load sets including thermal loads (NB-3654.2(b)).

\subsubsection{Non-reversing dynamic loads}

When Eq.(9) verified, the stress intensity limit is according to NB-3654.2 set to $1.8 S_{\mathrm{m}}$, but no greater than $1.5 S_{\mathrm{y}}$. Recall that it sets to $1.5 S_{\mathrm{m}}$ for Service limit Level A loads, implying a $20 \%$ relaxation of the stress intensity limit for Level B loads as compared to that for Level A loads.

Any direct instruction for further evaluation has not explicitly been given in NB-3654 and NB-3223 if Eq.(9) unsatisfied. We note that the first statement in NB-3654 is "The procedures for analysing Service Loadings for which Level B Service Limits are designated, are the same as those given in NB-3653 for Level A Service Limits". This should allow us, as we do for Level A loads, to apply NB-3200 to use a non-linear analysis to predict the collapse-load, or its lower bound, and the design can still be qualified if the applied loads are less than $2 / 3$ of the collapse-load. The remaining question is how various parameters, such as the yield stress and so on, should be set in a non-linear analysis.

If the collapse-load is predicted in accordance with the Plastic Analysis specified in NB3228.3, there will be no ambiguity as the true material yield stress and true stress-strain relation are used, see also Section 5.1. However, if a Limit Analysis is chosen, we may then ask: Should the yield stress be set to $1.5 S_{\mathrm{m}}$ as for Level A loads? Or should it be set to a value corresponding to the stress intensity limit $1.8 S_{\mathrm{m}}$ (but no greater than $1.5 S_{\mathrm{y}}$ ) that is used in connection with the linear design evaluation?

The authors favor to set the yield stress to $1.8 S_{\mathrm{m}}$ (but no greater than $1.5 S_{\mathrm{y}}$ ) based on the following "engineering" reasoning: (1) Setting $1.5 S_{\mathrm{m}}$ as the yield stress in a Limit Analysis for Level A loads is because the stress intensity limit for Level A loads sets to $1.5 S_{\mathrm{m}}$, which 
should be an important correlation between the linear and non-linear designs. (2)The linear and non-linear design principles can differ in many ways, but they are set in order to achieve, for an ideal design, the same safety margin. (3)The fact that the stress intensity limit for Level B loads is $20 \%$ relaxed as compared to that for Level A loads in a linear design should be "accounted or compensated" somewhere in its corresponding non-linear design, through e.g. raising the yield stress by $20 \%$ or, equivalently the factor $2 / 3$ to $1.2 \times 2 / 3=4 / 5$.

There are different views about the above choice in Sweden. Some colleagues advise that the yield stress must set to $1.5 S_{\mathrm{m}}$ in the Limit Analysis for all loads no matter which Service limits they are designated to. We will return to this issue in Section 5.2.

Remark: All load sets in Level A and B (both reversing and non-reversing) must be together taken into account when computing the cumulative usage factor $U$.

\subsubsection{Reversing dynamic loads}

The evaluation of the fatigue and thermal ratchet requirements are the same as those given in Section 3.2. Additionally, it is required (NB-3654.1(b)) that any deflection limit prescribed by the design specification must be met. Our suggestions for a non-linear evaluation are described in Section 3.2.3.

Remark: All load sets in Level A and $B$ (both reversing and non-reversing) must be together taken into account when computing the cumulative usage factor $U$.

\subsection{Level C}

The linear design evaluation for all load sets for which Service limit Level C is designated, is given in NB-3655. The evaluation rules are again given in terms of reversing and nonreversing loads.

We note in advance that for Service limit Level $C$ deformation limits prescribed by design specifications are explicitly required to be verified, see NB-3653.3. This is required for loads of both non-reversing and reversing types.

\subsubsection{Non-reversing dynamic loads}

For non-reversing loads, Eq.(9) in NB-3652 should be applied with a relaxed stress intensity limit $2.25 S_{\mathrm{m}}$, but no greater than $1.8 S_{\mathrm{y}}$, which is relaxed by $25 \%$ as compared to Service limit Level B.

If Eq.(9) unsatisfied, similarly to cases for Level B loads, any direct instruction for further evaluation has not explicitly been given in NB-3655 and NB-3224.

Referring to our discussion in Section 3.3.1 for Level B loads, it should be reasonable to use the same approach that handles Level B loads to do a further evaluation. That is, a nonlinear finite element analysis is used to predict the collapse-load or its lower bound. The design can still be qualified if the applied loads are less than $2 / 3$ of the collapse-load.

Again, if the collapse-load is predicted in accordance with the Plastic Analysis specified in NB-3228.3, there will be no ambiguity as the true material yield stress and true stress-strain relation are used. However, if a Limit Analysis is chosen, we may again ask: Should the yield stress be set to $1.5 S_{\mathrm{m}}$ as for Level A loads? Or should it be set to a value corresponding to the stress intensity limit $2.25 S_{\mathrm{m}}$ (but no greater than $1.8 S_{\mathrm{y}}$ ) that is used in connection with the linear design evaluation?

The author favor again, based on the same reasoning given in Section 3.3.1, the choice of setting the yield stress to $2.25 S_{\mathrm{m}}$ (but no greater than $1.8 S_{\mathrm{y}}$ ) or, equivalently setting the yield 
stress to $1.5 S_{\mathrm{m}}$ but increasing the factor $\frac{2}{3}$ to $\frac{2.25}{1.5} \times \frac{2}{3}=1.0$. There are different views on such a choice. A few co-workers believe that the yield stress should always be set to $1.5 S_{\mathrm{m}}$ in a Limit Analysis for all loads no matter which Service limits they are designated to, see a more in-depth discussion in Section 5.3.

\subsubsection{Reversing dynamic loads}

The evaluation rule for reversing loads is given in NB-3655.2(b). The evaluation is done by applying conditions given in NB-3656(b), which are given for loads in Service limit Level D. When applying these conditions, the stress intensity limit given in NB-3656(b)(2) remains the same, and those given in NB-3656(b) $(3,4)$ are reduced by $30 \%$. The fatigue evaluation is not required.

If the evaluation of NB-3656(b) disqualified, a further assessment can be done by applying the 5\% strain limit rule described in Section 3.2.3 without any modification. This follows from the following reasoning:

1. When NB-3656(b) cannot be fulfilled, one checks further the conditions in NB-3656(c). NB-3656(c) states that design rules in Appendix F can be used as an alternative to NB3656( $\mathrm{a}, \mathrm{b})$. One observes however that Appendix $\mathrm{F}$ is not specified for reversing loads.

2. Although no explicit rules found in Appendix F for reversing loads, one can fortunately find in NB-3228.6 the following statements: "As an alternative to meeting the requirements of Appendix F, for piping components subjected to reversing type dynamic loading ..., the requirements of (NB-3228.6) (a)(1) and (a)(2) below shall be satisfied".

3. NB-3228.6(a)(2) concerns the fatigue control which is not required for Level C loads. This means that only NB-3228.6(a)(1) needs to be followed.

4. NB-3228.6(a)(1) states that "The effective ratchet strain averaged through the wall thickness of the piping component due to the application of all simultaneously applied loading including pressure, the effect of gravity, thermal expansion ranges, earthquake inertia ranges, anchor motion ranges, (including thermal, earthquake etc.) and reversing dynamic loading ranges shall not exceed $5 \% "$. (Notice the badly formulated texts!)

Remark: There are different views on the above reasoning as Appendix $\mathrm{F}$ is not given for reversing loads. A few people argue that the only alternative to NB-3655.2 is the application of NB-3224.7, which requires fulfilling the requirements of through NB-3224.1 to NB-3224.6. It indicates in turn by NB-3224.7 that NB-3228 Plastic Analysis, with $70 \%$ of the specified allowable strain values, can be applied. Namely, we require (i) the maximum accumulated local strain being less than $0.7 \times 5 \%=3.5 \%$, and (ii) $\varepsilon_{a n} \leq 0.7 \cdot \frac{S_{a 10}}{E \sqrt{N}}$, see Section 3.5.2.

\subsection{Level D}

The linear design evaluation for all load sets for which Service limit Level D is designated, is done similarly to that specified for Service limit Level C, and the general evaluation rules are given in NB-3656. The evaluation rules are again specified in terms of the two types of loads as defined for Level B and C loads, i.e. non-reversing and reversing loads.

\subsubsection{Non-reversing loads}

For non-reversing loads, the linear evaluation rule is given in NB-3656 (a), which states that Eq.(9) in NB-3652 should be applied with a relaxed stress intensity limit $3.0 S_{\mathrm{m}}$, but no greater than $2.0 S_{\mathrm{y}}$. 
If Eq.(9) unsatisfied, NB-3656(c) can be applied, which in turn refers to Appendix F, indicating that a non-linear evaluation can be done through the prediction of the collapseload or its lower bound.

Appendix F states general rules and acceptance criteria for piping analyses when Service limit Level D considered. Roughly speaking, the requirements specified for Service limit Level D are relaxed as compared with Service limits of Level A, B and C. Below we shall have a close look at Appendix F.

The general acceptance criteria when material plasticity taken into account are given in F1340. It is stated (F-1341) that the acceptability may be demonstrated using one of the following methods: (a) Elastic analysis; (b) Plastic analysis; (c) Collapse-load analysis; (d) Plastic instability analysis; and (e) Interaction methods. This is, in our opinion, obviously not a consequent and clear statement.

First, the option (a) is no longer applicable when plasticity considered. Secondly, plasticity instability is a phenomenon that may for some cases, depending on both structure itself and applied load, not always occur and, for other cases, can definitively occur long before the applied load reaches its collapse point. Nevertheless, with reference to this statement and the evaluation rule for non-reversing loads in Service limit Level C, it should be a correct choice that we apply the option (c) Collapse-load analysis and, meanwhile, check if any plastic instability shall take place. We note these two options can be examined in one nonlinear analysis, see below.

F-1341.3 states in connection with the collapse-load analysis that: The applied static load, or its equivalent, should not exceed $100 \%$ of the collapse-load, or $90 \%$ of the lower bound of the collapse-load obtained in a limit analysis.

When the limit analysis used, the yield stress is according to F-1341.3 set to $\min \left(2.3 S_{\mathrm{m}}\right.$, $0.7 S_{\mathrm{u}}$ ), where $S_{\mathrm{u}}$ is the ultimate strength (A relaxation of about $2 \%$ as compared to Service limit Level C). Notice here that a different relaxation is used when setting the yield stress as compared to that used for the linear design evaluation, where the stress intensity limit is set to $3.0 S_{\mathrm{m}}$, that is, a relaxation of about $33 \%$ as compared to Service limit Level C. Apparently, the advice of setting the yield stress to $1.5 S_{\mathrm{m}}$ is not appropriate here.

F-1341.4 states that "the applied load should not exceed $70 \%$ of the so-called plastic instability load $P_{\mathrm{I}}{ }^{\prime \prime}$. Generally speaking, $P_{\mathrm{I}}$ can only be determined if an incremental solution, with both material plasticity (true stress-strain relationship) and large deformation taken into account, applied to numerically trace the response history. However, it is generally not an easy task from numerical point of view, and requires finite element software that are able to accurately handle various difficulties in so-called "path-searching", such as snap-back, snap-through and so forth, see Fig. 2, where local buckling or instability appears, resulting a temporally and partly lost of the load-carrying capacity. Notice that if thin-walled piping structures are under consideration, instability phenomena can in most cases occur before the collapse-load reached, and $P_{\text {I }}$ can then be much less than the collapseload if there exist any material or geometric imperfection. Hence, it is equally important to verify $P_{\mathrm{I}}$ and the collapse-load. Unfortunately, it is often the case that plastic instabilities cannot be accurately predicted and $P_{\mathrm{I}}$ cannot be observed in numerical results.

In Fig.2, two careless finite element (FE) solutions are shown. Both solutions fail to predict the plastic instability phenomena. While the solution which diverged early leads to a much conservative design, the other solution may result in a catastrophic design.

Fig. 2 also indicates that both the collapse-load and plastic instability load can be predicted in the same non-linear analysis through tracing the responses history. This implies that a 
collapse-load analysis should be the first choice. Whereas a limit analysis should be avoided when the non-reversing loading considered and Eq.(9) in NB-3652 unsatisfied. Otherwise, one cannot be sure if plastic instability is under control.

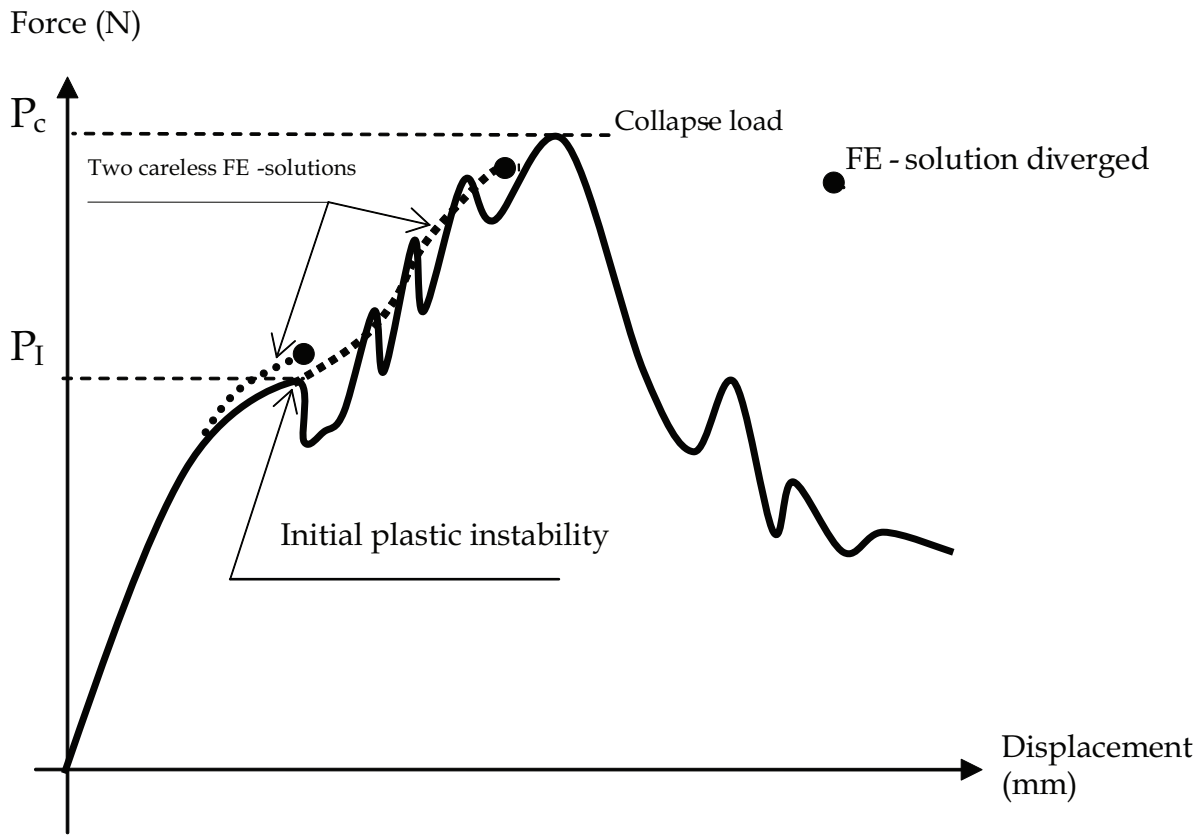

Fig. 2. Load-displacement relation including plastic instabilities typically observed in structures of thin-walled members and two careless FE-solutions

\subsubsection{Reversing dynamic loads}

For the reversing loading, the linear design evaluation is done by evaluating conditions given in NB-3656(b)(1)-(5). The fatigue evaluation is not required as for load sets in Service limit Level D.

If the linear design evaluation disqualified, a further assessment can be done according to NB3228.6 (a)(1)-(2). The design is qualified if

1. the $5 \%$ strain limit rule is satisfied, NB-3228.6(a)(1); and

2. a thermal ratchet limit is satisfied through NB-3228.6(a)(2)

$$
\varepsilon_{a n} \leq \frac{S_{a 10}}{E \sqrt{N}}
$$

Above, $\varepsilon_{a n}$ is an effective cyclic single-amplitude strain, $S_{a 10}$ is the allowable fatigue stress limit at 10 cycles according to the design fatigue curves given in ASME III Appendix I, and $E$ the Young's modulus, $N \geq 10$ the number of cycles for general reversing dynamic loads prescribed in design specifications, and $N=10$ for earthquake events.

For computing $\varepsilon_{a n}$ a procedure described in NB-3228.6(a)(2) should be applied. This procedure requires operating at material-points of interest, e.g. element's Gaussian points, 
where the results of strain components are available, over a typical load cycle which is considered to be of interest. Denote one chosen material-point by $p$ and the procedure can be summarized (in a standard tensor notation) as follows:

Step 1. Extract and record the strain results $\varepsilon_{i j}^{k}$ at all considered time-steps $k=1,2, \ldots, N$ in a complete load cycle of interest.

Step 2. Calculate the strain change $\Delta \varepsilon_{i j}^{k}$ between each time-step $k$ and a reference time-step $k_{0}$, e.g. $k_{0}=1$. That is, for each $k \neq k_{0}$, we calculate $\Delta \varepsilon_{i j}^{k}=\varepsilon_{i j}^{k}-\varepsilon_{i j}^{k_{0}}$.

Step 3. Calculate the (von Mises) equivalent strain change at time step $k \neq k_{0}$, i.e.

$$
\Delta \varepsilon_{e q}^{k}=\sqrt{\frac{2}{3} \Delta \varepsilon_{i j}^{k} \Delta \varepsilon_{i j}^{k}} \text {. }
$$

Step 4. Find the maximum equivalent strain range by

$$
\varepsilon_{\max }=\max \left(\Delta \varepsilon_{e q}^{k}\right), k=1,2, \ldots, N .
$$

Step 5. The effective cyclic single-amplitude strain is $\varepsilon_{a n}=\frac{1}{2} \varepsilon_{\max }$.

Notice that it is important to find the material-point at which the maximum equivalent strain range takes place. Notice that software e.g. ANSYS does not directly provide such output. Additional efforts must be made in order to evaluate this quantity.

\section{Class 2 and 3 piping}

The linear design evaluation rules for Class 2 are given in ASME III, NC-3652 - 3655 and relevant rules are given in other items in NC-3600. The following discussion will first be made by following the rules given in NC-3600. Thereafter, we describe alternative non-linear design evaluation rules for Class 2 piping.

The rules for Class 3 piping (ND-3600) are basically the same as those for Class 2 piping (NC-3600) and their difference is minor. They are, however, also applicable for Class 3 piping.

Similarly to Class 1 piping, different design requirements are, in general, specified for loads including non-reversing dynamic loads or those including reversing dynamic loads.

\subsection{Linear design evaluation}

The linear design evaluation rules for all load sets in Design Condition, Service Limits of Level A, B, C and D, are given in NC-3652 - 3655. These rules are summarized below. We remark in advance that, except for Service limit Level D, no further design assessment instruction has been provided if the linear design evaluation disqualified.

\section{Design condition}

For the Design Condition the effects of sustained loads should satisfy Eq.(8) in NC-3652 to ensure the primary stress intensity within its limit $1.5 S_{\mathrm{h}}$, where $S_{\mathrm{h}}$ is the basic material allowable stress at design temperature. In addition, the moment term $M_{\mathrm{A}}$ in Eq.(8) should be given based on conditions according to NCA-2142.1(c) Design Mechanical Loads.

We note that for Class 1 piping the stress intensity limits are always defined in term of $S_{\mathrm{m}}$. Notice the difference that for Class 2 the "hot" allowable stress $S_{h}$ is in use. This happens for all load conditions, see below. 


\section{Level A and B}

The design requirements for Levels $\mathrm{A}$ and $\mathrm{B}$ are given in a badly formulated text. In particular, requirements given in NC-3653.2 are confusing and can be interpreted in several ways. We agree the following interpretation:

For the Service limit Level B, the effects of sustained loads, occasional loads including nonreversing dynamic loads should satisfy Eq.(9) in NC-3653.1 to ensure the primary stress intensity within its limit $1.8 S_{\mathrm{h}}$.

For the Service limit of Levels A and B, the effects of thermal expansion should satisfy Eq.(10), and the effects of any single non-repeated anchor movement Eq.(10a), see NC3653.2. As an alternative to the fulfilment of, Eq.(10), Eq.(11) shall be satisfied.

For the Service limit of Levels A and B, the effect of reversing loads must always meet the condition given in Eq.(11a) in NC-3653.2(d).

\section{Level C}

For the Service limit Level C, the evaluation rules are also specified in terms the two types of loads as defined for Class 1 piping, i.e. non-reversing and reversing loads.

The effects of the non-reversing loads should satisfy Eq.(9) with a relaxed stress intensity limit $2.25 S_{\mathrm{h}}$ (but no greater than $1.8 S_{\mathrm{y}}$ ).

For the reversing loads, conditions given in NC-3655(b) should be satisfied, using the allowable stress in NC-3655(b)(2), and 70\% of the allowable stresses in NC-3655(b)(3-4). Furthermore, deformation limits given by design specifications should be verified.

\section{Level D}

For the Service limit Level D, the evaluation rules are again specified in terms of the two types of loads as defined for Class 1 piping, i.e. non-reversing and reversing loads.

NC-3655(a) requires that the effects of the non-reversing loads should satisfy Eq.(9) with a relaxed stress intensity limit $3.0 S_{\mathrm{h}}$ (but no greater than $2.0 S_{\mathrm{y}}$ ). For the reversing loads, conditions given in NC-3655(b) should be satisfied. NC-3655(c) states that "the rules given in Appendix F, where non-linear design requirements are given, can be used as an alternative to verify both non-reversing and reversing loads".

\subsection{Non-linear design evaluation}

The review that we made in Section 4.1 indicates that, except for Level $D$, no further evaluation instruction has been provided if the linear design evaluation disqualified. The question becomes: For other load sets, can we apply non-linear analyses to further assess the piping design as we do for Class 1 piping?

It has been discussed and argued that piping and vessels are similar, and one may apply NC-3200 Alternative Design Rules for Vessels to do such job. Hence, evaluation rules given in Appendix XIII, and in particular those given in Appendix XIII-1150 Plastic Analysis, Limit Analysis and Shakedown Analysis, can directly be used as advised in NC-3221.1.

We note that there is one major difference between design rules for Class 2 vessels (NC3300) and piping (NC-3600), see e.g. Slagis (1987). Vessels are required to meet stress limits on "primary" stresses only. Whereas for piping, thermal expansion stresses including concentration effects are explicitly evaluated against relevant limits through Eqs.(10-11), see NC-3653.2, which is a control on fatigue. From this point of view, it is not appropriate to apply NC-3200 Alternative Design Rules for Vessels for Class 2/3 piping. 


\subsubsection{A Class-upgrade alternative}

When Level D considered, the application of Appendix F to verify a Class 2 piping in cases when the linear design evaluation disqualified is, in fact, equivalent to consider the Class 2 piping as Class 1 . This can be straightforward realized by carefully examining how a Class 1 piping is verified for Level D, see Section 3.5. This observation is, to the authors' point of view, important as it implies two design principles for Level D when material plasticity taken into account:

1. The Class 2 piping is considered as a Class 1 piping.

2. The design requirements specified for Class 1 in accordance with the considered load set, without any modification/relaxation, are applied.

One may naturally ask why these principles are only applied to Level D, but not to all load sets. There are different guesses/explanations and attempts to justify them. To find the answer is not the scope of this report. We note only that the load sets in Level D includes loads resulted from the most extreme accidents e.g. the lost of coolant, leading generally to (large amount) plastic deformations, which implies consequently that the linear design evaluation that is purely based on stress limits becomes for some cases too easy to be violated, and can no longer play an appropriate role as a criterion to justify the acceptability of the piping design.

Our experiences have indicated that design evaluation for Class 2 piping with material plasticity taken into account for all other load sets has been of a great concern and become a natural request. Under the circumstances that no clear rules have been given for load sets of Design Condition and Levels A, B and C, we think it should be a reasonable alternative to apply the above two principles. One may argue that such an alternative is conservative and possible involves partly unnecessary work. To compromise these, we think it should be reasonable to partly introduce a "relaxation" in the second principle above.

\subsubsection{More on the Class-upgrade alternative}

The difference between the Class-upgraded alternative, discussed in the previous section, and the argued alternative discussed in Section 4.2.1, needs possibly to be further clarified. These two treatments are fundamentally different. To apply NC-3200 Alternative Design Rules for Vessels for Class 2/3 piping does not have a principal support. They are made for vessels and there are, as discussed earlier, differences between vessels and piping.

However, to raise a Class 2/3 piping to Class 1 does not fundamentally change the type of a structure, but only strengthens the design requirements or design safety considerations. The strengthened design safety will be loosen or, speaking in more appropriate words, balanced through relaxing those individual Class 1 design requirements. There may be many ways to relax those requirements and will, in some cases, have to find an "engineering" compromise between requirements for Class 1 and 2/3. The relaxation needs to be done on a base of concrete "individual case" and engineering judgments, which should be documented in detail. We believe that it coincides with the general design principles that ASME III has built.

\section{Collapse-load analysis}

As we discussed in Ch. 3 and 4, an alternative to the fulfilment of Eq.(9) in NB-3652 for Class 1 piping (Design, Level B, C and D), and of Eq.(9) in NC-3653 for Class 2/3 piping (Level D), is to apply a non-linear finite element analysis to predict the collapse-load, and to ensure 
that the applied load does not exceed a certain percentage of the collapse load. We shall here discuss such a non-linear evaluation in a more detailed setting.

One must first realize that a collapse-load analysis deals only with cases when applied loads are static, such as PD+DW and PO+DW+D/B shown in Tab. 1. We remember, however, that ASME III suggests that it may also be applied for cases where non-reversing dynamic loads are involved. We notice that a direct application of a collapse-load analysis when dynamic loads involved is not possible. In the following, we shall first focus us on cases with static loads.

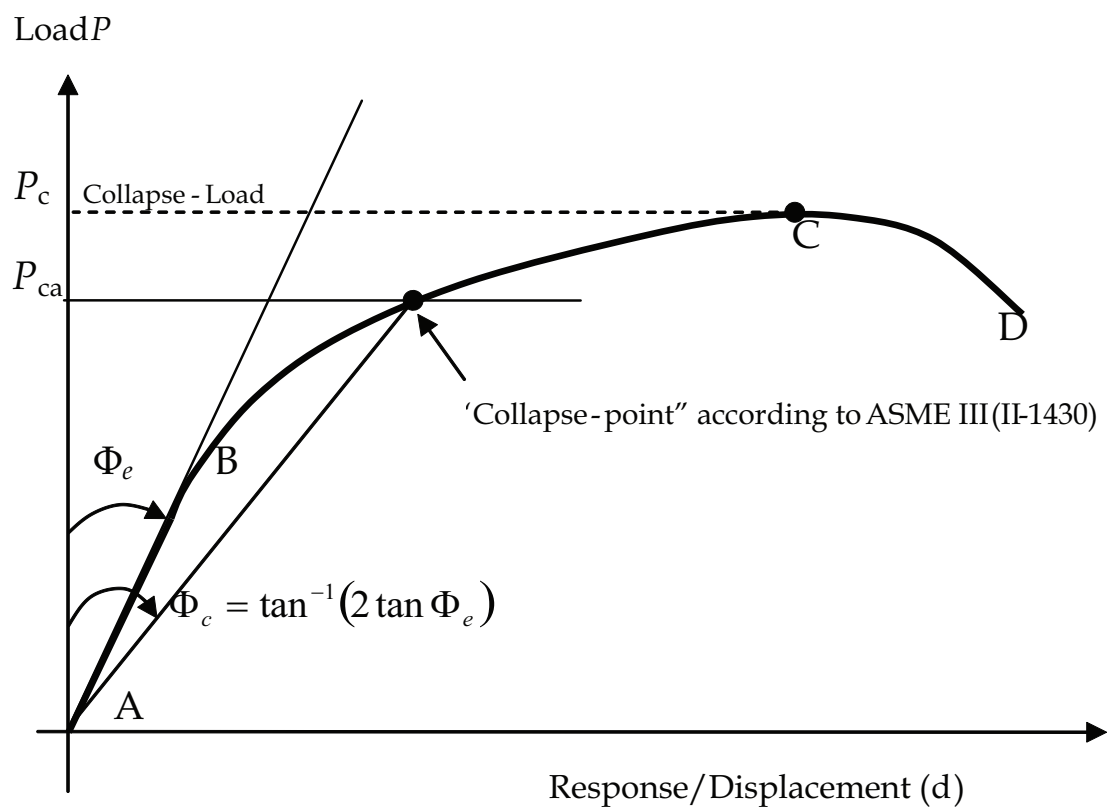

Fig. 3. Load-response history and the collapse-load

\subsection{General}

When plasticity and/or geometric non-linearities taken into account, a structure will loose its load-carrying capacity when the applied load $P$ reaches a critical level, or collapse-load, $P_{c}$. To determine the collapse-load, it is required to numerically predict the load-response history, see e.g. Fig. 3 for the simplest cases, from an early stage A until the collapse point C and, in many cases, until a stage $\mathrm{D}$ far after the collapse point. The numerical prediction of a load-response history in connection with finite element analysis is not a simple task as pointed out in Section 3.5.1 and relevant publications (Jansson, 1995). For a comprehensive discussion of corresponding computational procedures and numerical difficulties, we refer texts e.g. Bathe (1996) and Crisfield (1996). We remark the following:

1. At the collapse point the so-called tangent stiffness matrix is singular, implying usually a divergence of solution or computation. However, a diverged solution or computation does not necessarily imply that the collapse point has been reached or passed through. The divergence can be resulted by instability as mentioned in Section 3.5.1 or many other reasons. 
2. A load set includes generally several loads. When plasticity taken into account, the structural responses (deformation and stress state) depend on how and in what order these loads are applied.

3. The "collapse-load" defined in ASME III is generally less than the true collapse-load, ASME PVB Code, Section II-1430 (ASME, 2009b). This implies that one cannot determine the collapse-load by simply taking the load-level at which a computational divergence occurred, see also Fig. 2.

4. In practice, when a piping system found to be "overstressed" somewhere in the piping system, one attempts to avoid to analyze the whole piping system in a non-linear finite element analysis. (We do analyze the whole piping system in many cases.) Instead, a critical part, for example, a bend or a T-branch, where the maximum overstress taken place, is first identified, and "cut" out from the piping system. Thereafter, a refined finite element model using e.g. 3-dimensional or shell elements is built for this critical part. Finally, relevant displacement solutions on the "cut" faces from the linear analysis are used as boundary conditions for the refined finite element model. This means that, the collapse-load analysis is made on a component level.

\subsection{Plastic analysis according to ASME III}

The prediction of the collapse-load according to ASME III should be done in accordance with the Plastic Analysis specified in NB-3213.25, 3228.3 and Appendix II-1430. Below we first discuss the modeling issues and, thereafter, describe briefly how the "collapse" load according to NB-3213.25 can be determined.

NB-3228.3 states that the true material stress-strain relationship should be used. Explicitly, it means that the true yield stress and strain hardening rule should be used. It has been observed in earlier performed work that the material is modeled by specifying the following when using non-linear finite element software e.g. ANSYS: (1) the true yield stress in a vonMises material and, (2) a small plastic modulus (e.g. $10 \mathrm{MPa}$ ) in bilinear kinematical hardening. Strictly speaking, this is far away from what NB-3228.3 requests. In such a modeling, no hardening has been taken into account.

Notice that for some metals strain hardening is significant and, in addition, exhibits a strong Bauschinger's effect. In such cases, a correct prediction of the response history can most likely not be made without considering hardening effects. This will particularly be true if cyclic loading and shakedown process should be modeled, see Section 6. Intuitively, one may think that the prediction of the collapse-load is in nature static analysis, where external loads are increased incrementally and, hence, repeated unloading-loading processes are not involved. This leads, in turn, to a conclusion that hardening effects are not important. Such reasoning is fundamentally wrong. The following facts must be reminded: While increasing external loads, the development of plastic deformation somewhere in a structure, changes the way that the structure carries the external loads. Consequently, stresses in the structure must be redistributed. That is to say, stresses at some material-points will increase and at some other material-points decrease. In other words, some material-points undergo a loading process and some others an unloading process. The loading and unloading processes will, depending on the structure and applied loads themselves, repeatedly take place during the entire course of the development of plastic deformation.

NB-3228.3 suggests also taking large deformation into account in predicting the collapseload. This is explicitly required especially when Service limit Level D considered. For this case plastic instability should be examined, see Section 3.5.1. 
Again, we remind that the load-level, at which the computation diverges, cannot be considered as the collapse-load. Instead, a load-displacement curve should be plotted, see e.g. Figs. 2 and 3. Thereafter, the "collapse point" should be determined using a procedure described in NB-3213.25. In Fig. 3 this procedure is illustrated, where $P_{\text {ca }}$ and $P_{\mathrm{c}}$ stands for the "collapse" load according to ASME III and the true collapse-load, respectively. As illustrated, $P_{\mathrm{ca}}$ can be far less than the true collapse-load $P_{\mathrm{c}}$, which will definitively be the case if thin-walled structures dealt with.

\subsection{Limit analysis according to ASME}

The Limit Analysis described in ASME III differs from the Plastic Analysis discussed previously in two aspects: (1) In the Limit Analysis, an elastic-ideally-plastic material is assumed, and (2) the yield stress $\bar{\sigma}$ needs not necessarily be set to the true material yield stress $S_{\mathrm{y}}$, instead, to some allowable stress value which, for example, is $1.5 S_{\mathrm{m}}$ for Class 1 piping when Design Condition considered, and $\min \left(2.3 S_{\mathrm{m}}, 0.7 S_{\mathrm{u}}\right)$ for Class 1 piping when Level D loads considered.

In this sense, the limit analysis specified in ASME III provides only a useful estimation of the lower-bound of the collapse-load. Other related results, e.g. plastic strains at particular material points, are much less reliable and, thus, should not be used for decisive judgement purposes.

We have mentioned earlier that the setting of the yield stress in a Limit Analysis has only been explicitly stated in ASME III for two cases: Class 1 piping when loads of Design Condition considered, and Class 1 piping when Level D considered. We have suggested that, for other cases, the yield stress can be set to the stress limit value that is used in connection with the linear design evaluation. Namely, we suggest to set $\bar{\sigma}$ for Class 1 piping to $1.5 S_{\mathrm{m}}, \min \left(1.8 S_{\mathrm{m}}, 1.5 S_{\mathrm{y}}\right), \min \left(2.25 S_{\mathrm{m}}, 1.8 S_{\mathrm{y}}\right), \min \left(2.3 S_{\mathrm{m}}, 0.7 S_{\mathrm{u}}\right)$ for Design, Level $\mathrm{B}, \mathrm{C}$ and $\mathrm{D}$ loads, respectively. In such a way, the yield stress $\bar{\sigma}$ depends on the piping Class, the load set under consideration, and the design requirement (equation number) which is not satisfied in the linear design evaluation. And so will be the predicted collapse-load.

Suppose that a piping system is subjected to a non-reversing load $P$, which should be considered as a load in four different conditions: Design, Level B, C, and D conditions, respectively. The above suggestion can be more clearly illustrated in Fig. 4 , where $P_{A}, P_{B}, P_{C}$ och $P_{D}$ denotes collapse-loads are predicted in the Limit Analyses.

In Fig. 4 we also illustrate the consequence if the yield stress is always set to $1.5 S_{\mathrm{m}}$ in the Limit Analysis. That is, it always requires $P \leq \frac{2}{3} P_{A}$ no matter which Service limits a load $P$ is designated to.

Alternatively, as discussed in Sections 3.3.1 and 3.5.1, we may set the yield stress $\bar{\sigma}$ to $1.5 S_{\mathrm{m}}$ in the Limit Analysis and, instead of using the factor $\frac{2}{3}$ when determine the "collapse-load", we use a "relaxed" factor, $\frac{4}{5}$ (for Level B loads) and 1.0 (for Level C loads).

In a common engineering language, the design philosophy may be interpreted as below: Under a normal operating condition (Level A), stresses in piping components shall be kept low within elastic range. In connection with emergency events (Level C), various components can be subjected to so high stresses that those components, which undergo a sufficiently high deformation, may continue to be used if certain specific tests can be passed. 
In connection with faulted events (Level D), components which undergo a sufficiently high deformation should be replaced by new components. We consider that our suggestions coincide with the design philosophy upon which AMSE III has been built.

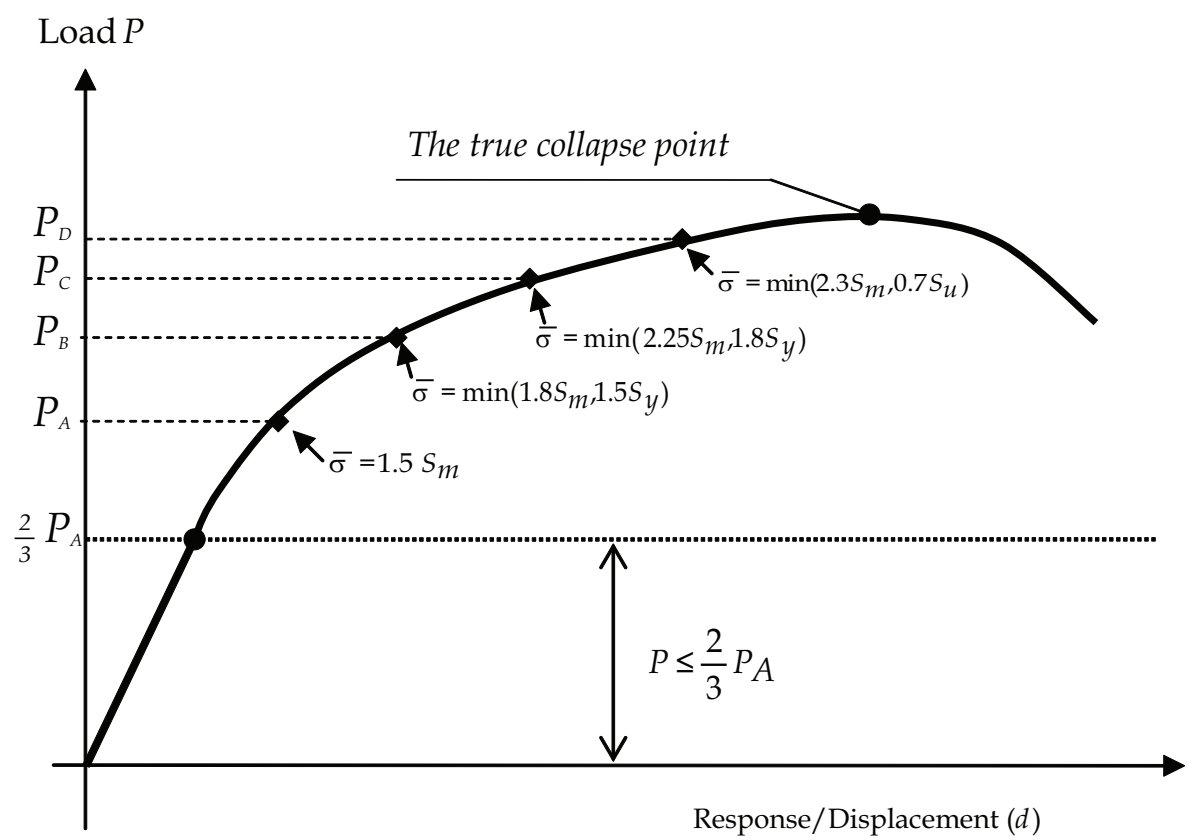

Fig. 4. Principal sketch of using a Limit Analysis to predict the collapse-loads for Design, Level $B, C$, and $D$, when yield stresses set to different $\bar{\sigma}$

\section{Non-linear transient analysis}

For reversing loads, a non-linear evaluation requires generally to use a non-linear finite element analysis to trace transient structural responses. This is directly applicable for all load cases which do not include any dynamic load defined by floor response spectra.

For such cases, the first essential goal of the evaluation is for most cases to examine if the $5 \%$ strain limit rule can be satisfied. When material plasticity involved, the non-linear transient analysis should be conducted with direct integration algorithms such as Newmark's integration, see e.g. Bathe (1996) and Crisfield (1996), as the tangent stiffness (matrix) has to be updated at each time-increment. Notice that it is the Plastic Analysis specified in NB3213.25 that we conduct in a non-linear transient analysis, which implies that the true material stress-strain relationship, i.e. the true yield stress and the true strain hardening behavior, should be used.

Unlike a collapse-load analysis which can be conducted on a component level, a non-linear transient analysis must always be conducted on the whole system level. Furthermore, when the non-linear analysis is made on the whole piping system, it is normally not possible to model all components with sufficient accuracy, as too simple element models may be used for certain components, for example, T-branches and bends. In such cases, in addition to the 
non-linear transient analysis, one needs possibly cut these components out from the whole piping system and try to find their equivalent "static problem" and to predict their "equivalent" collapse-loads.

In non-linear transient analysis, one focuses on historic transient responses, such as transient stresses and strains. Hence, the use of realistic non-linear material models is of vital importance. Among several important issues, the strain hardening behavior of piping materials have been intensively discussed in recent years.

The ultimate strength of the many materials that are listed in ASME is about twice as much as their initial yield strength and, for some exceptional cases, more significant hardening effects can be observed. For example, the yield stress is $35 \mathrm{ksi}$, whereas the ultimate strength reaches $90 \mathrm{ksi}$ for materials SB-581 through SB-626, see Tab.1B, Division II, Part D (ASME, $2009 \mathrm{~b}$ ). To predict a correct transient response, the strain hardening effect is an important part in a non-linear transient analysis as cyclic loading and possibly a shakedown process are of main concern.

The strain hardening behaviour is better illustrated in Fig. 5, where two typical hardening rules, i.e. isotropic and kinematic rules, associated with von Mises yield criteria are shown on a deviatoric plane. In isotropic hardening, the von Mises yield surface expands in the radial direction only during the development of the plastic deformation. (The "initial" cylinder expands and forms the "current" one.) In kinematic hardening, however, the size and shape of the yield surface remain unchanged, but the centre of the yield surface (the central axis of the cylinder) moves during the development of the plastic deformation. (The "initial" one moves and forms the "current" one.) In this way, the kinematic hardening rule allows to include the Bauschinger's effect. There is a third available rule which is a combination of the isotropic and kinematic rules, and requires a more elaborated material test-data when it should be used.

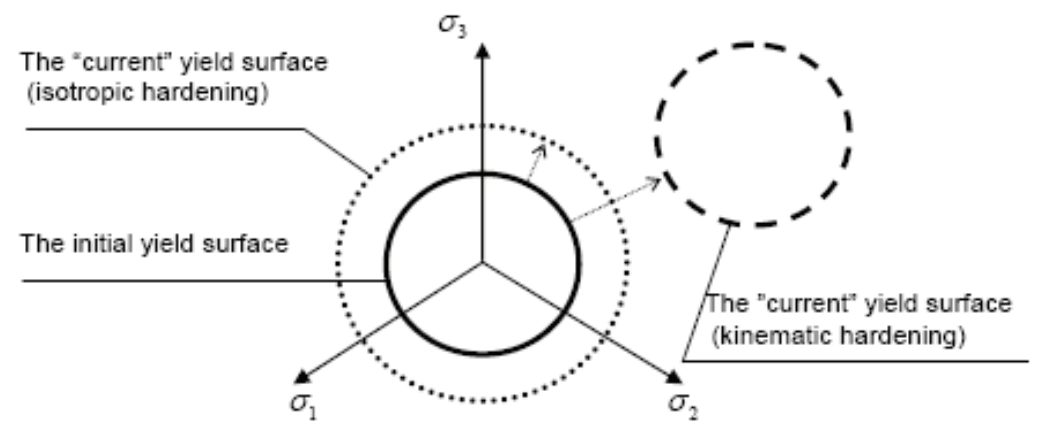

Fig. 5. Isotropic and kinematic hardening behavior on a deviatoric plane

Linear or multi-linear kinematic hardening models in commercial finite element software, e.g. ANSYS or others, are frequently found to be used for non-linear piping analysis. It has been, however, shown in recent reports by Rahman et al. (2008), Hassan et al. (2008) and Krishna et al. (2009) that such non-linear finite element analyses can only provide a reasonable modeling of plastic shakedown phenomena after a few initial load cycles. For continuous ratcheting responses, such analyses cannot provide reasonable results, neither for the accumulated local strain nor for the global dimension change. They showed through experiments on straight and elbow pipe components that several nonlinear constitutive 
models available in most general finite element software, such as Chaboche (1986), Ohno and Wang (1993), and other more recently developed models (Abdel Karim and Ohno, 2000; Bari and Hassan, 2002; Chen and Jiao, 2003) can provide a much improved prediction.

\section{Concluding remarks}

We have in this chapter categorized the design evaluation given in ASME III for nuclear piping of Class 1, 2 and 3 into the linear design and non-linear design evaluations. The corresponding design requirements, in particular, those non-linear design requirements, have in the report been reviewed, analyzed and clarified in association with every defined load set, through Design Condition to Service Limit Level D. Efforts have been made to formulate the non-linear design evaluation requirements in a format so that they are easy to be followed, understood and applied in connection with piping analysis.

The non-linear design evaluation requires in principle two types of non-linear finite element analyses: collapse-load analysis and non-linear transient analysis. We have in the chapter attempted to describe in detail their computational aspects in a close accordance with the requirements given in ASME III.

The design requirements given in ASME III for nuclear piping have been developed in more than several decades. However, it has been a known issue that its formulation and specification of design requirement items are far from fully clear, which are caused by endlessly nested references in multiple levels to a large amount of contents. This is, unfortunately, particularly true when design-by-analysis rules are considered. We hope this chapter should be able to serve as a constructive source for a better understanding of and a potential improvement for the design requirements for nuclear power piping.

\section{Acknowledgement}

This work is partially funded by ÅFORSK through Agreement Ref. No. 10-174, which is gratefully acknowledged.

\section{References}

Abel Karim, M. and Ohno, N. (2000). Kinematic hardening model suitable for ratcheting with steady state, Int. J. Plasticity, 16, 225-240.

ANSYS, Inc., (2010). ANSYS Mechanical - Users' Manual (Version 13), USA.

ASME (2009a). The American Society of Mechanical Engineers, ASME Boiler \& Pressure Vessel Code, Section III, Division 1 - Subsections NB, NC, ND, NCA and Appendices.

ASME (2009b). The American Society of Mechanical Engineers, ASME Boiler \& Pressure Vessel Code, Section II, Part D.

Bathe, K. J. (1996). Finite Element Procedures, Prentice Hall, Englewood Cliffs, NJ.

Crisfield, M. A. (1996). Non-Linear Finite Element Analysis of Solids and Structures. Vol. 1 Essentials. Wiley Professional, UK.

Bari, S. and Hassan, T., (2002). AN advancement in cyclic plasticity modeling for multiaxial ratcheting simulation, Int. J. Plasticity, 18, 873-894.

DST Computer Services S.A., (2005). PIPESTRESS User's Manual, Version 3.5.1, 2005.

Slagis G. S. \& Kitz, G. T. (1986). Commentary on Class 1 piping rules, PressureVessels, Piping and Components - Design and Analysis, ASME PVP, Vol. 107, 1986. 
Jansson, L. G. (1995). Non-linear analysis of a guide and its stitch welds for repeated loading, Computers \& Structures, Vol. 56, No. 2/3.

Krishna, S., Hassan, T., Naceur, I. B., Sai, K., and Cailletaud, G., (2009). Macro versus microscale constitute models in simulating proportaional and non-proportional cyclic and ratcheting responses of stainless steel 304. Int. J. Plasticity, 25, 1910-1949.

Ohno, N. and Wang, J. D. (1993). Kinematic hardening rules with critical state of dynamic recovery - Part I: formulation and basic features for ratcheting behavior. Int. J. Plasticity, 9, 375-390.

Rahman S. M., Hanssan, T and Corona, E. (2008). Evaluation of cyclic plasticity models in ratcheting simulation of straight pipes under cyclic bending and steady internal pressure", Int. J. Plasticity, 24, 1756-1791.

Slagis, G. S. (1987). Commentary on Class 2/3 piping rules, Design and Analysis of Piping, PressureVessels and Components (Eds: W. E. Short II, A.A: Dermenjian, R.J. McGrattan and S.K. BHandari), ASME PVP, Vol. 120.

Zeng, L., (2007). Design verification of nuclear piping according to ASME III and required nonlinear finite element analyses. (Internal report), ÅF-Engineering AB, Sweden.

Zeng, L., Horrigmoe, G. and Andersen, R., (1996). Numerical implementation of constitutive integration of rate-independent plasticity, Int. J. Comput. Mech., Vol. 18, No. 5.

Zeng, L. and Jansson, L. G., (2008). Non-linear design verification of nuclear power piping according to ASME III NB/NC, Proc. 16 th Int. Conf. Nuclear Eng. (ICONE16), Orlando, USA.

Zeng, L., Jansson, L. G. and Dahlström L. (2009). More on non-linear verification of nuclear power piping according to ASME III NB/NC, Proc. 17th Int. Conf. Nuclear Eng. (ICONE17), Brussels, Belgium.

Zeng, L., Jansson, L. G. and Dahlström L., (2010). On fatigue verification of Class 1 nuclear power piping according to ASME III NB-3600. Proc. 18th Int. Conf. Nuclear Eng. (ICONE18), Xi'an, China. 


\title{
The Text-Mining Approach Towards Risk Communication in Environmental Science
}

\author{
Akihide Kugo \\ Japan Atomic Energy Agency \\ Japan
}

\section{Introduction}

As the failure of waste management had endangered the public safety, public concerns and awareness regarding waste disposal facilities which may bring dioxin pollution risk, PCB risk and other toxic threat have grown so much. A long-life radioactive waste disposal facility also becomes one of the public concerns. As the high level radioactive waste is not so familiar with the public, it brings the sense of fear of unidentified materials among local. Therefore, the site selection of high level radioactive waste (HLW) final disposal facility faces much difficulty in the world except in Finland and Sweden.

If concerns of environmental topics of the daily life could be properly connected with nuclear power issues, people would certainly be easy to participate in the discussion about the necessity of such facilities.

Therefore, the author investigated the relationship between the nuclear power issues and environmental topics such as household waste management or the precautionary principle analyzed by text-mining method. In this method, the author conducted the investigation cooperated with university students as subjects. The elements of this experiment consist of lectures on environmental topics, keywords of each lecture submitted by the students, and questionnaire survey result on nuclear power generation answered by the students.

Many researches on the risk communication regarding nuclear power issues have been implemented. For example, Kugo analyzed the public comments and discussion by using a text mining method (Kugo, 2005, 2008). Yoshikawa also introduced the researches on the human interface of the computer-aided discussion board (Yoshikawa, 2007). These researches aimed to grasp the representativeness of the public opinion by analyzing majority of the subjects.

However, the problem that the research data were not necessary reliable in term of the representativeness of the public because of the fluctuations of subjects' opinion existed. For example, a person has the tendency to make a decision in a heuristic way in case of requiring a prompt answer. Therefore, the new point of the method of this analysis was that the author did not include the information of the majority of the subjects but the minority based on the assumption that the reliance of the information of minority subjects was higher than those of the majority since the minority submitted the keywords without heuristic decision making. 


\section{Method and result of analysis}

First, the author gave lectures on the risk perception and desirable autonomous ideas in the area of various environmental sciences including nuclear power generation issues at a university class. Students submitted a keyword that they considered as the best representative for each lecture. The keywords submitted were classified into two groups by cluster analysis and correspondence analysis on the keywords-subjects cross table. These analyses result to calculate the eigenvalue of the cross tabulation.

On this calculation process, every small part of the keywords-subjects cross table called a cluster. A relative relation of a cluster could be grasped, plotting two compounds of the eigenvalue of clusters on the $x-y$ axis position. Chi-square distance could easily be calculated by using these $x-y$ data. By chi-square distance from the centre, it could be majored of the representativeness of the students.

This result of the analyses indicated that the keywords of frequent occurrence locate near the centre of the chart and the keywords of less frequent occurrence locate at a circumference part. Based on the keyword cluster deployment on the chart and its characterization, the arrangement of the keyword cluster can be interpreted along with the assumed mental model.

Students whose consciousness level was low would choose keywords that were easy to find through the lectures (lecture titles, word appeared on the delivered documents, etc.). In that case, the frequency of chosen keywords would be high because those keywords were limited to in the documents. On the other hand, students whose consciousness level was a little higher would choose keywords that were emotional or used in the discussion during the lectures. If these keywords depended on the students internal idea, not limited to in the documents, the frequency of these keywords occurrence would be less than that of keywords chosen by low-consciousness level students. Thus, the author paid more attention to the less frequency keywords and students who submitted these keywords.

Second, the author conducted the questionnaire research pertaining nuclear power generation and high level radioactive waste (HLW) disposal management at the end of all lectures. The concepts of the questionnaire consisted of necessity, approval for facility installation, and acceptance of adjoining facility. The students selected number of answer from "yes" to "no" by seven grades. Consequently, two groups of the students above described were characterized by ANOVA (Analysis of Variance) respectively. One was passive, and the other was active toward the attitude of acceptance of a nuclear facility.

Third, by using keyword cross table, the author analyzed the correlation between the keyword groups of the lecture at each theme. Thus, the communication points could be extracted by paying attention to the correspondence of the pair of keywords chosen at two themes of lectures. In this paper, the author shows the results of two cases such as keywords group of the theme of nuclear power generation and household waste management, and the theme of nuclear power generation and the precautionary principle as examples. The concept of this correlation analysis shows in Figure 1.

\subsection{Lectures on environmental science and keywords and assumed mental model}

The students received the series of fifteen lectures (ninety minutes per a lecture) on environmental science. In these lectures, they discussed various themes such as global warming, waste problem, ozone hole, dioxin poison, radioactivity, precautionary principle, 
and some other themes. The basic concept of these discussions was that we should have objective viewpoint not to avert the risk but to face it. After every lecture, students submitted the most impressive keyword in the theme with a message of the reason. The number of keywords was one hundred and sixty seven in total. The effective number of students who attended the whole lecture was fifty.

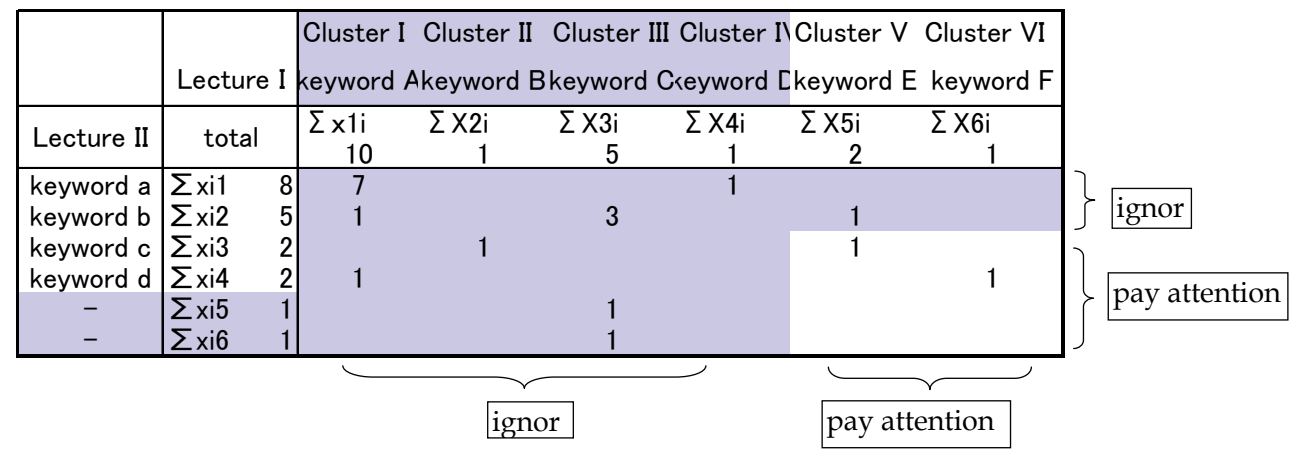

Fig. 1. Concept of the keyword cross table analysis by the keywords of two lectures

Table 1 gives the themes of fifteen lectures and the number of the submitted keywords at every lecture. In this research of the relationship between the theme of "nuclear power generation" and "household waste management" and the relationship between the theme of "nuclear power generation" and "the precautionary principle", the author tried to find the students' common value in their internal mind. Table 2 shows the submitted keywords at above designated three lectures.

\section{Theme of Lecture}

\begin{tabular}{|c|c|c|}
\hline & Theme of Lecture & Number of submitted keyworc \\
\hline \# 1 & System of global environment & 21 / 54 students \\
\hline$\# 2$ & Global warming & / 55 \\
\hline \# 3 & Precautionary principle & / 57 \\
\hline \# 4 & Dioxin & / 55 \\
\hline \# 5 & Household Waste management & / 55 \\
\hline \# 6 & Ecological footprint & $/ 56$ \\
\hline \# 7 & Ozone hole & / 53 \\
\hline \# 8 & Energy & / 53 \\
\hline \# 9 & Radioactivity & / 53 \\
\hline \# 10 & Nuclear power generation & / 50 \\
\hline \# 11 & Earthquake & / 49 \\
\hline \#12 & Environmental Sociology & / 46 \\
\hline \# 13 & Safety and Relief & 10 / 49 \\
\hline \# 14 & Others & - \\
\hline \#15 & Questionnaires survey & - \\
\hline
\end{tabular}

Table 1. Theme of lectures and the number of submitted keywords at every lecture 


\begin{tabular}{lll}
$\begin{array}{l}\text { lecture on } \\
\text { Nuclear Power generation }\end{array}$ & $\begin{array}{l}\text { lecture on } \\
\text { Househould Waste management }\end{array}$ & $\begin{array}{l}\text { lecture on } \\
\text { the Precautionary principle }\end{array}$ \\
\hline Friburg (the name of city) & 3R(Reduce, Reuse, Recycle) & Zero risk \\
MOX Fuel utilization in LWRs & Quantity of disposal waste & Dioxin \\
Nuclear fuel cycle & Incentive & Dioxin news report \\
Nuclear Power generation & Globalization & Risk \\
Nuclear energy revolution & Discharge of the waste & Problem of risk \\
Insecurity or understanding among citizen & Plastics & Risk communication \\
Renewable energy & Recycle & Risk management \\
Public opinion poll & Circulative society & Risk information \\
Radioactive waste & Disposal cost & Risk cognition \\
& Thermal supply system & Risk analysis \\
& Waste & Environmental hormone \\
& Responsibility for disposal & Dioxin concentration \\
& Illegal disposal & Precautionary principle \\
\hline
\end{tabular}

Table 2. The keywords at the designated lecture

The assumed basic mental model that consists of "instinct (inner part of mind)", "emotion (middle part of mind)", and "reason (outer part of mind) shows in Figure 2.

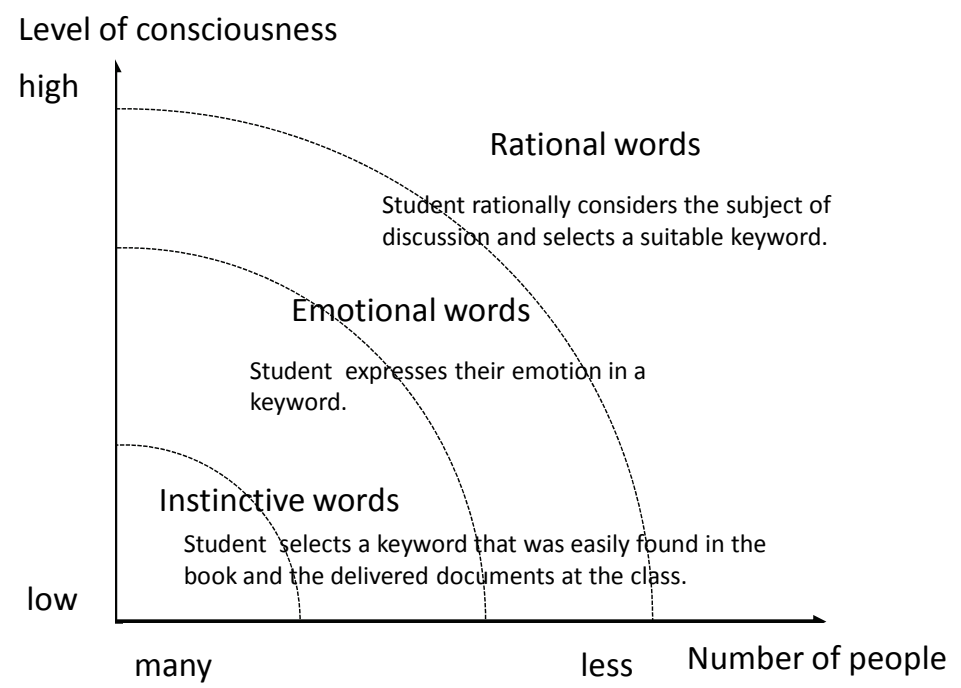

Fig. 2. The mental model of keywords chosen at the lecture (assumption)

If a student whose consciousness level was low submitted a keyword by request, he would try to choose a keyword that was easy to find through the lectures (lecture titles, words appeared in the book or the delivered documents, etc.). This action should be the appearance of representative heuristic decision making, in other words. Consequently, the frequency of occurrence of the keywords would be high.

On the other hand, students whose consciousness level was higher than the former would choose keywords that were emotional or used in the discussion time. The frequency of occurrence of these keywords would be less than that of keywords of low-consciousness level students. These words were not limited to in the documents but depended on the 
students' internal idea. If a student's consciousness level were much higher than the other levels, the frequency of their keywords would be less than those of keywords of students whose consciousness levels were low or middle, since their keywords choice based on their own opinion.

After the lecture on nuclear power generation, the author implemented correspondence analysis and cluster analysis on the basis of "keywords - subjects cross table" in order to apply the assumption of the above described mental model.

If the mental model were well, the words chosen by many students would be the title of the lecture (i.e. nuclear power generation). The number of students who chose the keyword of "nuclear power generation" was twenty-seven, which was the most. The number of students who chose the keyword of "MOX Fuel utilization in LWRs" was eleven, which was the second. The numerical information about the number of keywords submitted in the lecture titled "nuclear power generation" shows in table 3.

In accordance with the assumed mental model, the keyword of "Insecurity or understanding among citizen", "Renewable energy", Public opinion poll", and "Nuclear energy revolution" might carry the subjective image or the meaning of something emotional. Conversely, "Nuclear power generation" and MOX fuel cycle", "radioactive waste" and "Friburg" might carry the objective image or neutral meaning. However, this understanding remains vague for the student classification. Therefore, in order to classify these keywords along with above described mental model, the author implemented text-mining analysis described next section.

Keywords of the lecture titled by "nuclear power generation" Number of subjects

\begin{tabular}{lc}
\hline Nuclear power generation & 27 \\
MOX Fuel utilization in LWRs & 11 \\
Nuclear fuel cycle & 3 \\
Radioactive waste & 3 \\
Friburg (name of the city) & 2 \\
Insecurity or understanding among citizen & 1 \\
Natural renewable energy & 1 \\
Public opinion poll & 1 \\
Nuclear energy revolution & 1 \\
\hline
\end{tabular}

Table 3. Numerical information of keywords of the lecture on nuclear power generation

\subsection{Text mining for keywords}

The method of textual data mining was useful for analyzing public opinion. Ohsumi and Levert reported the results of textual data mining method (Ohsumi and Levert, 2000). The summary of the text mining method that consists of cluster analysis and correspondence analysis shows below.

Every lecture gave the information of keyword list and their occurrences. This frequency of occurrence data calls a contingency table. This " $\mathrm{m} \times \mathrm{n}$ " contingency table indicates frequencies of the appearances of " $n$ " different keywords of " $m$ " different students in the class. 
In other words, the co-occurrence data represent a matrix $\mathrm{X}$ which has $m$ rows and $n$ columns, or " $m \times n$-dimensional vector $\mathbf{F}$ ".

$$
\underset{m \times n}{\mathbf{F}}=\left(f_{i j}\right) \quad\left(f_{i j} \geq 0, i \in I, j \in J\right)
$$

$I=\{1,2, \ldots, m\}, J=\{1,2, \ldots, n\}$

By using Chi-square statistics, the dimension of deviations from the expected values can be identified.

Profiles:

diag : diagonal matrix

$$
\begin{aligned}
& P_{I J}=\left(P_{i j}\right) \quad(i \in I, j \in J) \\
& P_{I}=\operatorname{diag}\left(P_{i+}\right) \quad(i \in I) \\
& P_{J}=\operatorname{diag}\left(P_{+j}\right) \quad(j \in J)
\end{aligned}
$$

$$
\left(\begin{array}{l}
P_{i j}=\frac{f_{i j}}{N}, N=\sum_{i=1}^{m} \sum_{j=1}^{n} f_{i j}\left(\equiv f_{++}\right) \\
P_{i+}=\frac{f_{i+}}{N}=\frac{\sum_{j=1}^{n} f_{i j}}{N}, P_{+j}=\frac{f_{+j}}{N}=\frac{\sum_{i=1}^{m} f_{i j}}{N}
\end{array}\right)
$$

The data matrix (Matrix $\mathbf{X}(\mathbf{m}, \mathbf{n})$ ) shows below based on above matrix or two-way table.

$$
\underset{m \times n}{\mathbf{X}}=\left(x_{i j}\right) \quad(i \in I, j \in J) \quad x_{i j}=\frac{p_{i j}}{p_{i+} \sqrt{p_{+j}}}-\sqrt{p_{+j}}=\frac{q_{i j}}{\sqrt{p_{+j}}}-\sqrt{p_{+j}}
$$

Matrix $\mathbf{X}(\mathrm{m}, \mathrm{n})$ is the same for the equation below.

$$
\underset{m \times n}{\mathbf{Q}}=\left(y_{i j}\right) \quad(i \in I, j \in J) \quad y_{i j}=\frac{p_{i j}}{\sqrt{p_{i+} p_{+j}}}=\frac{f_{i j}}{\sqrt{f_{i+} f_{+j}}} \quad\left(p_{i+} \neq 0, p_{+j} \neq 0 ; f_{i+} \neq 0, f_{+j} \neq 0\right)
$$

Then, Matrix $\mathbf{Q}(\mathbf{m}, \mathbf{n})$ replaced like below.

$$
\underset{m \times n}{\mathbf{Q}}=\mathbf{P}_{I}^{1 / 2} \mathbf{P}_{I J} \mathbf{P}_{J}^{1 / 2}
$$

Consequently, Matrix V $(\mathbf{m}, \mathbf{n})$ attributes to extracting an eigenvalue.

$$
\underset{m \times n}{\mathbf{V}}=\mathbf{Q}^{\prime} \mathbf{Q}=\mathbf{P}_{J}^{-1 / 2} \mathbf{P}_{J I} \mathbf{P}_{I}^{-1} \mathbf{P}_{I J} \mathbf{P}_{J}^{1 / 2} \quad\left(\begin{array}{c}
\mathrm{Q}^{t}=\text { transposed matrix } \mathrm{Q} \\
\mathrm{P}_{J I}=\text { transposed matrix } \mathrm{P}_{I J}
\end{array}\right)
$$

Row and column coordinates can be plotted on the single screen by using component scores such as $\left(Z_{i k}, Z_{i k^{\prime}}\right)$ and $\left(Z_{i k}^{*}, Z_{i k^{\prime}}^{*}\right)$.

$$
\begin{aligned}
& z_{i k}=\frac{1}{\sqrt{\lambda_{k}}} \sum_{j=1}^{n}\left(\frac{P_{i j}}{P_{i+}}\right) z_{j k}^{*} \quad(i \in I, k=1,2, \ldots, K) \\
& z_{j k}^{*}=\frac{1}{\sqrt{\lambda_{k}}} \sum_{i=1}^{m}\left(\frac{P_{i j}}{P_{+j}}\right) z_{i k}(j \in J, k=1,2, \ldots, K) \quad \lambda \text { : eigenvalue }
\end{aligned}
$$

The graphic presentation based on above calculation clearly shows the relationships between the keywords and students, with distance on the map being a representation of correspondence. 
Such plotting does not indicate the relationship between row points and column points but only the distances between row and column points.

The result of analysis shows Table 4 that indicates the numerical information of the clusters and Table 5 that indicates the numerical information of the keywords. It also illustrates on the graphs shown in Figure3 and Figure 4. As shown in Table 4 and Table 5, fifty students were divided into five groups; the nine keywords were divided into five clusters in other words. Namely, The largest cluster of "I" (Cluster I) contains two keywords such as "the nuclear power generation" (theme of the lecture) and "MOX Fuel utilization in LWRs (Pluthermal)" chosen by thirty-eight students. It should be safe to say that Cluster I represented the group of students who had chosen instinctive keywords. Therefore, Cluster I located in the vicinity of the centre by Chi-Square Distance (0.20).

The second cluster of "II" (Cluster II) contained only a keyword such as "insecurity or understanding among the citizen" that was chosen by a student. This cluster located far from the centre by Chi-Square Distance (3.68).

The third cluster of "III" (Cluster III) contained two keywords such as "nuclear energy revolution" and "Freiburg" that were chosen by three students. This cluster located more distant from the centre by Chi-Square Distance (5.57).

The fourth cluster of "IV" (Cluster IV) that contained three keywords such as "renewable energy", "radioactive waste" and "public opinion poll" that were chosen by five students. This cluster located far from the centre by Chi-Square Distance (7.92).

The fifth cluster of "V" (Cluster V) that contained only a keyword such as "nuclear fuel cycle" that was chosen by three students. This cluster located in the longest distance from the centre by Chi-Square Distance (10.07).

The relation between the Chi-Square Distance of each cluster and the number of student that belonged to the cluster shows in Figure 5.

Then, author interpreted the meaning of cluster deployment on the screen as follows.

The keyword of the title of the lecture ("nuclear power generation") was chosen heuristically by most of the students. Therefore, the students who belong to the Cluster I did not have considered the theme so seriously. The author concluded Cluster I as an instinctive group. The keywords of "insecurity/understanding among citizen" (Cluster II) and "nuclear energy revolution" (Cluster III) could be holding the connotation of unstable condition. Students who belonged to these clusters must have expressed their emotion towards the subject of discussion. Thus, Cluster II and Cluster III that contained the emotional keywords were categorized into non rational groups. On the other hand, Cluster IV that contained the keywords of "renewable energy", "radioactive waste", "public opinion poll", and Cluster V that contained "nuclear fuel cycle" hold no subjective message. Students who belonged to these clusters must have grasped the topic of discussion and have expressed their result of consideration. Thus, the author concluded these Cluster IV and Cluster $\mathrm{V}$ as rational groups.

The curve of Number of Subjects - Chi square Distance relationship shown in Figure 5 had the consistency with the assumption of the mental model shown in Figure 2.

As the author considered the assumed mental model fit well, the author could classify the students into two groups along with the cluster deployment to investigate the attractive discussion points. The students who belonged to "Cluster I", "Cluster II", and "Cluster III" named the Passive group. The students who belonged to "Cluster IV" and "Cluster V" named the Active group. The concept of this classification shows in Figure 6. 
The author investigated the difference in an attitude between Active group and Passive group by using questionnaire survey, which referred nuclear power generation and radioactive waste management, as described in the next section.

\begin{tabular}{lcccccc}
\hline & $\begin{array}{c}\text { Number of } \\
\text { subjects }\end{array}$ & $\begin{array}{c}\text { Chi-squqre } \\
\text { distance }\end{array}$ & x-axis & y-axis & $\begin{array}{c}\text { Variation } \\
\text { within a } \\
\text { cluster }\end{array}$ & $\begin{array}{c}\text { Portion of } \\
\text { cluster } \\
\text { size }\end{array}$ \\
\hline Cluster I & 38 & 0.20 & -0.11 & 0.44 & 0.011 & 0.22 \\
Cluster II & 1 & 3.68 & 1.47 & -1.24 & 0.000 & 0.11 \\
Cluster III & 3 & 5.57 & -2.31 & -0.46 & 0.015 & 0.22 \\
Cluster IV & 5 & 7.92 & 0.02 & -2.81 & 0.017 & 0.33 \\
Cluster V & 3 & 10.07 & 3.17 & 0.04 & 0.000 & 0.11 \\
\hline total & 50 & - & - & - & 0.032 & 1.00 \\
\hline
\end{tabular}

Table 4. Numerical information of the clusters of the keywords at the lecture on "nuclear power generation", based on the cluster analysis

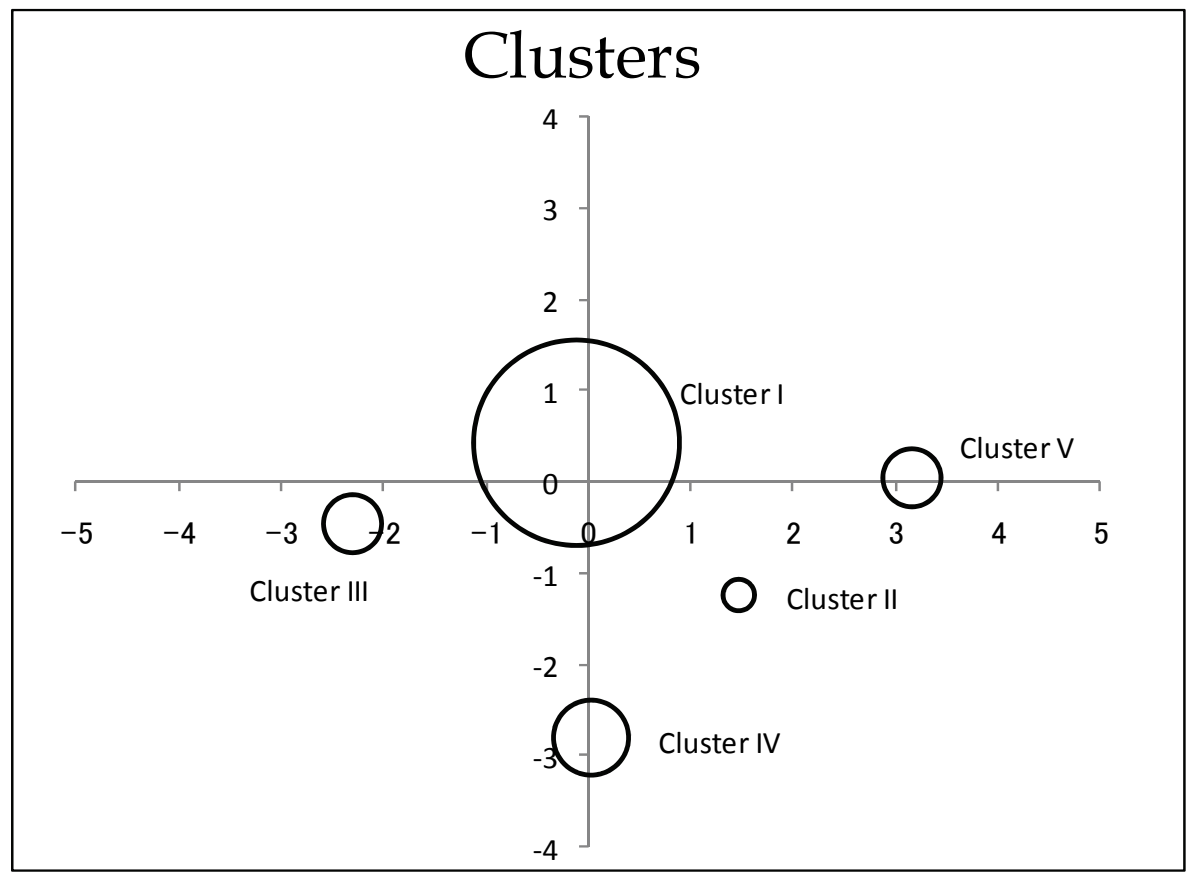

Fig. 3. Cluster deployment based on the cluster analysis of the keywords submitted at the lecture on "nuclear power generation". The area of the circle shows the number of students 


\begin{tabular}{lcrrrrrl}
\hline \multicolumn{1}{c}{ Keyword } & $\begin{array}{c}\text { Portion of Number } \\
\text { keyword } \\
\text { size }\end{array}$ & $\begin{array}{c}\text { Chi- } \\
\text { subjects }\end{array}$ & $\begin{array}{c}\text { square } \\
\text { distance }\end{array}$ & x-axis & y-axis & Cluster \\
\hline Nuclear Power generation & 0.54 & 27 & 0.9 & -0.08 & 0.51 & \\
$\begin{array}{l}\text { MOX Fuel utilization in } \\
\text { LWRs (Plu-thermal) }\end{array}$ & 0.22 & 11 & 3.6 & -0.18 & 0.27 & Cluster I \\
\hline Insecurity or understanding & 0.02 & 1 & 49.0 & 1.47 & -1.24 & Cluster II \\
\hline Nuclear energy revolution & 0.02 & 1 & 49.0 & -1.74 & -0.04 & Cluster III \\
Friburg (name of city) & 0.04 & 2 & 24.0 & -2.60 & -0.67 & \\
\hline Renewable energy & 0.02 & 1 & 49.0 & -0.13 & -2.39 & \\
Public opinion poll & 0.02 & 1 & 49.0 & 0.68 & -3.12 & Cluster IV \\
Radioactive waste & 0.06 & 3 & 15.7 & -0.15 & -2.85 & \\
\hline Nuclear fuel cycle & 0.06 & 3 & 15.7 & 3.17 & 0.04 & Cluster V \\
\hline \multicolumn{1}{c}{ total: 9} & 1.00 & 50 & - & - & - & \\
\hline
\end{tabular}

Table 5. Numerical information based on the cluster analysis of the keywords submitted at the lecture on "nuclear power generation"

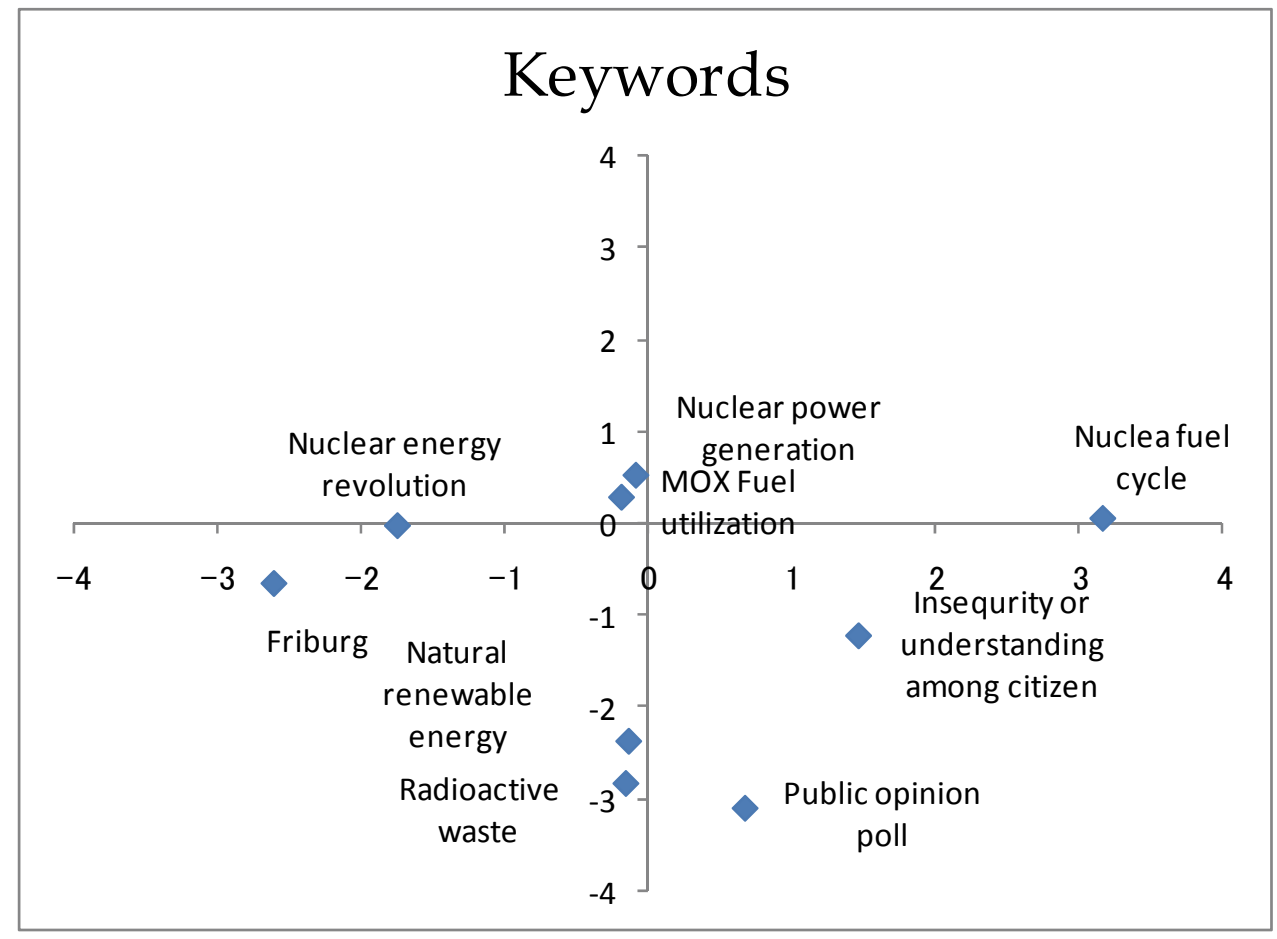

Fig. 4. Keywords deployment based on the cluster analysis of the keywords submitted at the lecture of "nuclear power generation" 


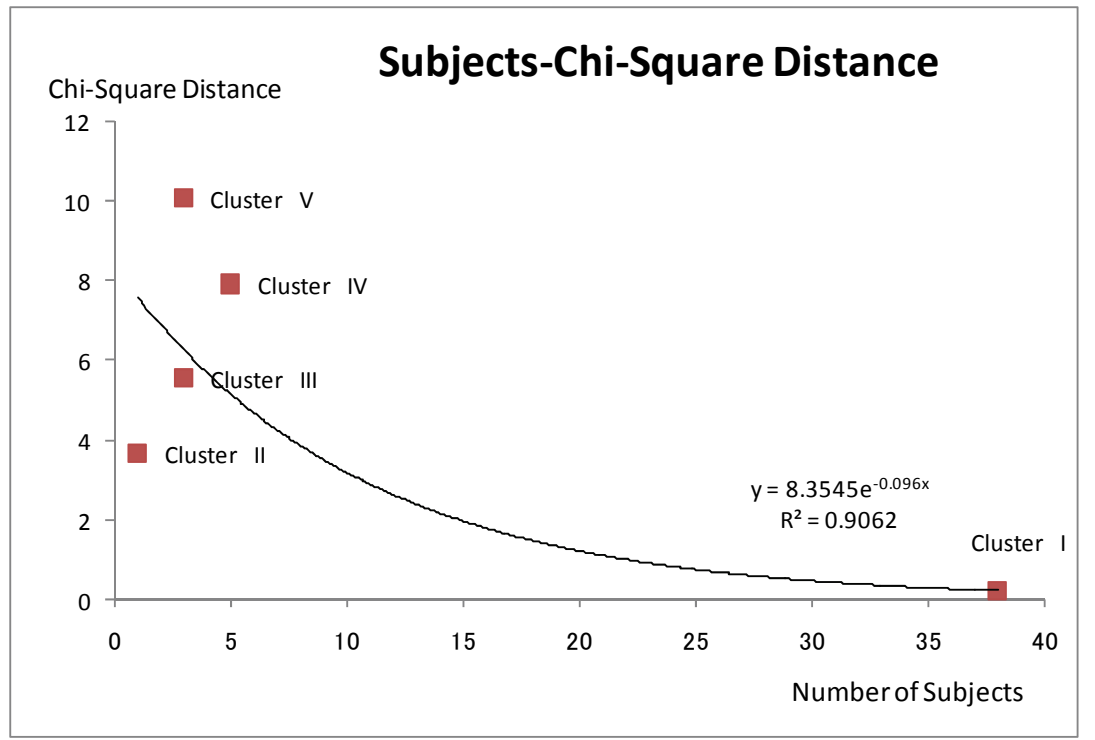

Fig. 5. Cluster deployment on the screen of number of Subjects vs. Chi Square Distance

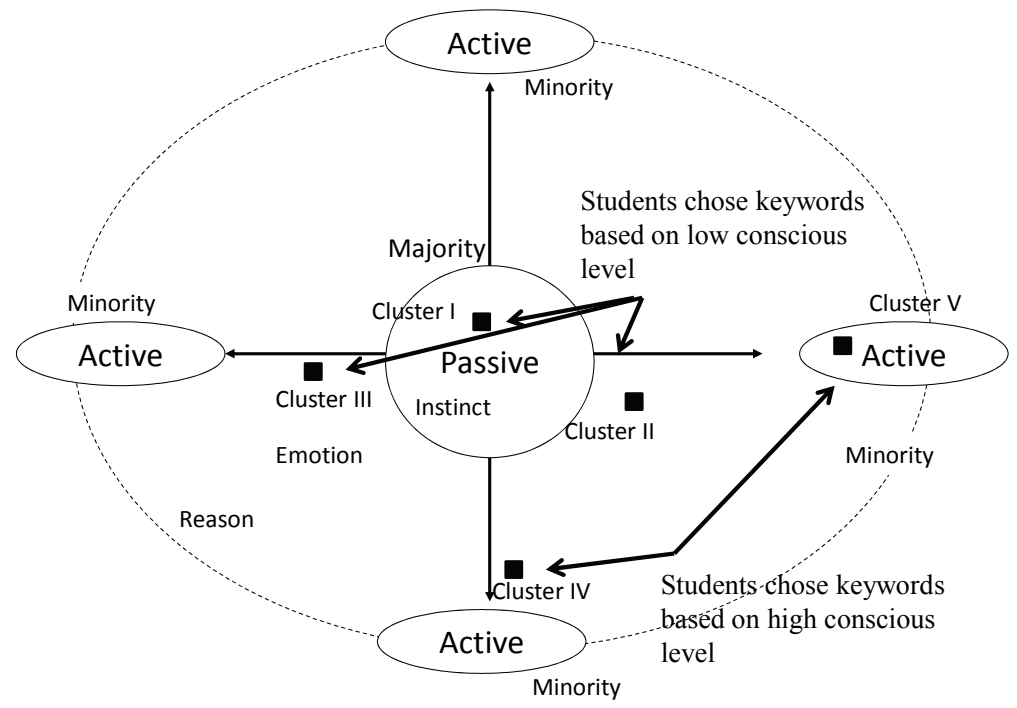

Fig. 6. The concept of the student classification

\subsection{Questionnaire survey on nuclear power generation and HLW}

At the last lecture, the students answered the questionnaire survey pertaining to the nuclear power generation and HLW (high level radioactive waste) disposal site selection in order to characterize the passive and the active group. 
The questionnaire composed of these questions from A to $G$ as below. The students selected the number from 7 (yes, I guess so very much) to 1 (no, I do not guess so at all) at every question.

Questionnaire A: necessity of nuclear power generation;

"Do you think nuclear power generation is necessary?"

Questionnaire B: approval for facility installation of nuclear power generation;

"Do you think it is no problem for the installation of nuclear power generation plant?

Questionnaire C: accepttance of adjoining nuclear power generation facility;

"Would you say "yes" if the local authority proposed you the construction of a nuclear power generation plant adjacent tot your place of residence?"

Questionnaire D: cognition of high level radioactive waste;

"Do you recognize HLW generated in the nuclear power plant?"

Questionnaire E: necessity of HLW disposal facility;

"Do you think the HLW disposal facility is necessary?"

Questionnaire F: approval for facility installation of HLW;

"Do you think it is no problem for the HLW disposal facility?"

Questionnaire G: acceptance of adjoining HLW disposal facility;

"Would you say "yes" if the local authority proposed you the construction of a geological disposal site adjacent to your place of residence?"

The author implemented ANOVA to find the significant difference between above two groups by using numerical answer of the questionnaires.

The result showed in Table 6, which revealed that there were no significant difference between the two groups in the consciousness toward nuclear power generation and necessity of HLW disposal facility and approval for the facility installation. However, there was a significant difference in the reluctant consciousness against adjoining facility installation.

This result suggested that the Active group in the theme of nuclear power generation had the positive stance toward the waste management of nuclear power generation. They felt the responsibility for the back-end procedure, in other words.

The students who belonged to the Active group believed that they had to face both side of science and technology, such as a benefit and disadvantage of nuclear power generation. They thought that nuclear power generation had the merit for energy security and environmental preservation. Conversely, they thought that it had the demerit of the requirement for long-life management of the high level radioactive waste.

On the other hand, the students who belonged to the Passive group did not have such a subjective attitude.

It would be the first to make an effort to share the feelings between a speaker and a listener (students of the passive group) by beginning the topics about their interest. If a speaker succeeded in getting the listeners' trust, a speaker would be easy to discuss the point of that theme. However, as the students of Passive group chose the keywords heuristically, it was difficult to get their interests. Therefore, the author paid attention to the keywords of other themes chosen by the Active group toward the HLW site selection. 


\begin{tabular}{cccc}
\hline \multicolumn{4}{c}{ Nuclear power generation } \\
\hline $\begin{array}{c}\text { Items of } \\
\text { questionaire } \\
\text { survey/group }\end{array}$ & $\begin{array}{c}\text { Necessity } \\
\text { of } \\
\text { Nuclear power } \\
\text { generation }\end{array}$ & $\begin{array}{c}\text { Approval } \\
\text { for } \\
\text { facility } \\
\text { installation }\end{array}$ & $\begin{array}{c}\text { Accept } \\
\text { of } \\
\text { adjoining facility }\end{array}$ \\
\hline Passive group & $5.5(1.20)$ & $3.9(1.25)$ & $2.8(1.67)$ \\
Active group & $5.8(0.87)$ & $4.3(0.99)$ & $3.3(1.31)$ \\
\hline significance & n.s. & n.s. & n.s. \\
\hline
\end{tabular}

The numerical value in each group indicate the average of ordinal scale of approval, such as " I guess so very much (7 point)", "neutral (4 point)", "I don't guess so at all (1point)" respectivly. The numerical value in a parenthesis shows standard deviation.

* : significant, $p<0.05$

\begin{tabular}{lcccc}
\hline $\begin{array}{l}\text { Items of } \\
\text { questionaire } \\
\text { survey/group }\end{array}$ & $\begin{array}{c}\text { Cognition } \\
\text { of } \\
\text { HLW }\end{array}$ & $\begin{array}{c}\text { Necessity } \\
\text { of } \\
\text { disposal facility }\end{array}$ & $\begin{array}{c}\text { Approval } \\
\text { for } \\
\text { facility } \\
\text { installation }\end{array}$ & $\begin{array}{c}\text { Accept } \\
\text { of } \\
\text { adjoining } \\
\text { facility }\end{array}$ \\
\hline Passive group & $3.9(0.64)$ & $4.3(0.71)$ & $3.4(0.92)$ & $1.9(1.13)$ \\
\hline \multicolumn{1}{c}{ significance } & $3.5(0.88)$ & $4.4(0.65)$ & $3.9(0.68)$ & $3.1(1.25)$ \\
\hline
\end{tabular}

The numerical value in each group indicate the average of ordinal scale of approval, such as "I guess so very much (7 point)", "neutral (4 point)", "I don't guess so at all (1point)" respectivly. The numerical value in a parenthesis shows standard deviation.

* : significant, $p<0.05$

Table 6. Result of ANOVA on the questionnaire survey pertaining to the nuclear power generation and HLW

The fifth theme of the lecture, household waste management, was a suitable issue to find the common element between the nuclear power generation and household waste management. The third theme of the lecture, precautionary principle, was also taken to find the common element. Because the characteristics to avert the risk at first would bring the chaos into the discussion of the site selection of HLW disposal, it would be difficult to achieve the social consensus.

\section{Discussion}

\subsection{The attitude for the environmental scientific-related theme}

In order to grasp the communication viewpoints, the author investigated the interest of the Active group in the field of environmental science such as the household waste management and the precautionary principle by using keyword cross table and correlation analysis.

\subsubsection{Correlation with the keywords at the lecture on the household waste management and those on nuclear power generation}

As shown in the Table 7 of the cross table which shows the keyword group obtained in the lecture of nuclear power generation and the lecture of household waste management, 
twenty six of the students (the largest number of the students) submitted the keyword of "nuclear power generation". The eleven students (the second largest number of the students) submitted the keyword of "MOX fuel (plutonium-uranium mixed fuel utilization) "at the lecture of nuclear power generation.

At the fifth lecture of household waste management, seventeen of above mentioned students who had selected "nuclear power generation" and "MOX fuel (plutonium-uranium mixed fuel utilization) " submitted the keyword of "recycle" and four of above mentioned submitted the keyword of "waste". These two keywords of "recycle" and "waste" were also top two of the submitted keywords at the lecture of general waste management.

This indicated that the students who had chosen the most and the second most keywords of both lectures did not consider these topics rationally. Therefore, it can be safe to say that the students who selected these top two of the keywords tended to make a decision in a heuristic way. They can be subordinate to the theme of the lecture in other words.

On the other hand, eight students identified as the member of Active group by the analysis of questionnaire survey chose the keywords such as "fuel cycle", "radioactive waste", "public poll", and "renewable energy" at the lecture of nuclear power generation. They chose seven keywords such as "3R (Reduce, Reuse, Reduction)", "globalization"," costs of waste management", "disposal" and other keywords. Two students, who belonged to the Active group, did not express the positive attitude toward the problem solution in the field of the household waste management, since they chose the keyword of "recycle" in the heuristic way.

Therefore, the author investigated the keywords of the students who belonged to the Active group of Cluster IV and Cluster V by considering the connotation of the six keywords of the lecture on the household waste management.

The students who had an interest in "nuclear fuel cycle" had paid attention to "3R (Reduce, Recycle, Reuse)," and "expense of waste management" in the area of household waste management. The students who had an interest in "radioactive waste" had paid attention to "globalization" and "waste disposal".

"Nuclear fuel cycle" and "3R" implied the common image of the recycling process, and "Disposal cost (expense of waste management)" implied the economic viewpoint. Therefore, this suggested that the students had a deep interest in the economic issue when they considered the flow of household waste, or radioactive waste.

The concept of the global relationships between the waste discharging country and the waste reprocessing country could be extracted from three keywords ("radioactive waste", "globalization" and "discharge of the waste"). This suggested that the students had a deep interest in the international relationships when they considered the process of the waste management.

The other students who had an interest in "renewable energy" paid attention to "Calculative society (the society which put emphasis on recycling)", and those who had an interest in "public opinion poll" paid attention to "rubbish discharge quantity."

From the keyword of "renewable energy" and "calculative society (the society which put emphasis on recycling)", the concept of the sustainable society could be extracted. The concept that the public had an interest in the process of decision making could be extracted from the keyword of "public opinion poll" and "quantity of disposal waste", considering the current social trend that public require the residential opinion poll for unpleasant facilities site selection. 
This suggested that special emphasis would be placed on the economical viewpoint, global relationships, sustainability, and the way of expression of individual opinion, when the students consider the problem solving toward HLW site selection. The correspondence of keywords between the area of "household waste management" and "nuclear power management" shows Figure 7.

\begin{tabular}{|c|c|c|c|c|c|c|c|c|c|c|}
\hline & & $\begin{array}{c}\text { Nuclear } \\
\text { Power } \\
\text { generation }\end{array}$ & $\begin{array}{l}\text { MOX Fuel } \\
\text { utilization } \\
\text { in LWRs }\end{array}$ & $\begin{array}{c}\text { Insecurity or } \\
\text { understanding } \\
\text { among citizen }\end{array}$ & $\begin{array}{c}\begin{array}{c}\text { Nuclear } \\
\text { energy } \\
\text { revolution }\end{array} \\
\end{array}$ & Friburg & $\begin{array}{c}\text { Renewable } \\
\text { energy }\end{array}$ & $\begin{array}{c}\text { Public } \\
\text { opinion } \\
\text { poll } \\
\end{array}$ & $\begin{array}{c}\text { Radioactive } \\
\text { waste }\end{array}$ & $\begin{array}{l}\text { Nuclear } \\
\text { fuel cycle }\end{array}$ \\
\hline Cluster & & $\mathrm{I}$ & $\mathrm{I}$ & II & III & III & IV & IV & IV & $\mathrm{V}$ \\
\hline total & $49^{* 1}$ & $26^{* 1}$ & 11 & 1 & 1 & 2 & 1 & 1 & 3 & 3 \\
\hline Recycle & 21 & 12 & 5 & & 1 & 1 & & & $1^{* 2}$ & $1^{* 2}$ \\
\hline Waste & 4 & 3 & 1 & & & & & & & \\
\hline Plastics & 4 & 2 & 2 & & & & & & & \\
\hline Circulative society & 4 & 3 & & & & & 1 & & & \\
\hline Disposal cost & 3 & 1 & & & & 1 & & & & 1 \\
\hline 3R(Reduce,Reuse,Recycle) & 2 & & 1 & & & & & & & 1 \\
\hline Globalization & 2 & & 1 & & & & & & 1 & \\
\hline Discharge of the waste & 2 & 1 & & & & & & & 1 & \\
\hline Responsibility for disposal & 2 & 1 & & 1 & & & & & & \\
\hline Illegal disposal & 2 & 1 & 1 & & & & & & & \\
\hline Quantity of disposal waste & 1 & & & & & & & 1 & & \\
\hline $\begin{array}{l}\text { Incentive } \\
\text { Thermal supply system }\end{array}$ & $\begin{array}{l}1 \\
1\end{array}$ & $\begin{array}{l}1 \\
1\end{array}$ & & & & & & & & \\
\hline
\end{tabular}

*1 A student, who was present at the lecture on nuclear power generation, was absent at the lecture of household waste management.

*2 omitted from the objects of analysis

Table 7. Cross table of the keywords at the lecture on "nuclear power generation" and "household waste management"

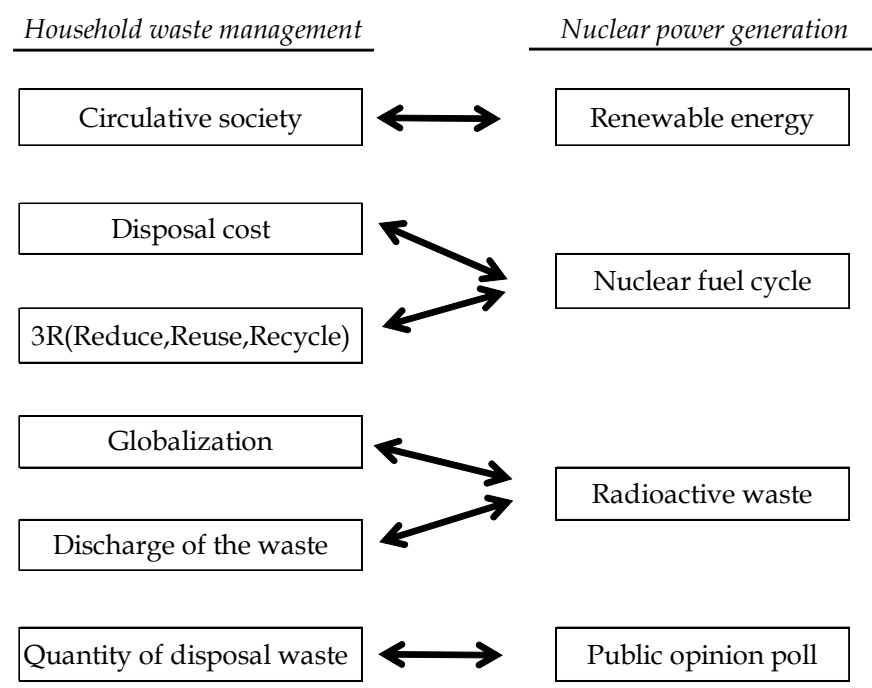

Fig. 7. Correspondence of the keyword (household waste management vs. nuclear power generation) 


\subsubsection{Correlation with the keywords at the lecture on the precautionary principle and those on nuclear power generation}

As shown in the Table 8 of the cross table which shows the keyword group obtained at the lecture of nuclear power generation and the lecture of the precautionary principle, eight students out of twenty seven students who had chosen "nuclear power generation" chose the keyword of "risk" at the lecture of the precautionary principle. Two students out of eleven students who had chosen "MOX fuel (plutonium-uranium mixed fuel utilization)" also chose the keyword of "risk". Six students out of twenty seven students who had chosen the "nuclear power generation" chose the keyword of "risk information" at the lecture of the precautionary principle. One of eleven students who had chosen "MOX fuel (plutoniumuranium mixed fuel utilization)" also chose the keyword of "risk information".

According to the above mentioned mental model, the students who chose these keywords of "risk" and "risk information", which were the top two of the selected keywords at the lecture of the precautionary principle, had the characteristics of decision making in the heuristic way. "The precautionary principle", which ranked the fourth place of the number of chosen keywords, was the title of the lecture". Consequently, the author decided to exclude the students who chose this keyword from the objects of analysis.

Therefore, the students, who chose the most and the second most keywords of lectures, and the student, who chose "the precautionary principle", tended to make a decision in a heuristic way. They could be subordinate to the theme of the lecture in other words.

On the other hand, eight students identified as members of Active group, who belonged to the Cluster IV and V by the analysis of questionnaire survey, had chosen the keywords of "fuel cycle", "radioactive waste", "public poll", and "renewable energy" at the lecture of nuclear power generation.

The Active group chose six keywords of "zero risk", "dioxin", "risk", "risk information" "dioxin concentration (in foods)" and "the precautionary principle" without their biasing on a particular keyword. Five out of eight students, who had been categorized as the Active member toward the HLW site selection, did not express the positive attitude pertaining to the problem solution in the field of the precautionary principle. They had just chosen the keyword of "risk", "risk information" and "the precautionary principle", which were chosen in a heuristic way by the large number of students.

On the other hand, three students who had chosen "public poll" and "radioactive waste" chose the keywords of "zero risk", "dioxin", and "dioxin concentration (in foods)" which were minor selections at all. They did not make a decision in a heuristic way but made rational consideration on the theme of nuclear power generation and the precautionary principle respectively.

When extracting the common underlying meaning from these non heuristic keywords such as "radioactive waste", "public poll", anti-centred policy style could be seen in the concept of demerit of the burden of nuclear power generation and the concept of individual opinion expression. When extracting the common underlying meaning from these non heuristic keywords such as "zero risk", "dioxin" and "dioxin concentration in foods", analytical or scientific attitude could be seen in the concept of quantitative thinking based on the numerical keyword such as the word of "zero" and "concentration".

This suggested that the students would have the antipathy toward logical thinking with an upper class viewpoint, the scientific and analytical viewpoint, when they considered the problem solving toward the HLW disposal site selection. The correspondence of keywords 
between the area of "the precautionary principle" and "nuclear power management" shows Figure 8.

\begin{tabular}{|c|c|c|c|c|c|c|c|c|c|c|}
\hline & & $\begin{array}{c}\text { Nuclear } \\
\text { Power } \\
\text { generation }\end{array}$ & $\begin{array}{c}\text { MOX Fuel } \\
\text { utilization } \\
\text { in LWRs }\end{array}$ & $\begin{array}{c}\text { Insecurity or } \\
\text { understandin } \\
\mathrm{g} \\
\text { among citizen }\end{array}$ & $\begin{array}{l}\text { Nuclear } \\
\text { energy } \\
\text { revolution }\end{array}$ & Friburg & $\begin{array}{c}\text { Renewable } \\
\text { energy }\end{array}$ & $\begin{array}{c}\text { Public } \\
\text { opinion poll }\end{array}$ & $\begin{array}{c}\text { Radioactiv } \\
\text { e } \\
\text { waste }\end{array}$ & $\begin{array}{c}\text { Nuclear fuel } \\
\text { cycle }\end{array}$ \\
\hline Cluster & & I & I & II & III & III & IV & IV & IV & $\mathrm{V}$ \\
\hline total & 50 & 27 & 11 & 1 & 1 & 2 & 1 & 1 & 3 & 3 \\
\hline Risk & 14 & 8 & 2 & 1 & & 1 & $1^{* 1}$ & & $1^{* 1}$ & \\
\hline Risk information & 9 & 6 & 1 & & & & & & & $2^{* 1}$ \\
\hline Dioxin news report & 6 & 3 & 3 & & & & & & & \\
\hline Precautionary principle & 5 & 1 & 3 & & & & & & & $1^{*} 1$ \\
\hline Risk management & 4 & 3 & 1 & & 1 & & & & & \\
\hline Risk cognition & 3 & 2 & & & & 1 & & & & \\
\hline Risk communication & 2 & 1 & & & & & & & & \\
\hline Environmental hormon & 2 & 1 & 1 & & & & & & & \\
\hline Dioxin concentration & 2 & 1 & & & & & & 1 & & \\
\hline Problem of Risk & 1 & 1 & & & & & & & & \\
\hline Zero Risk & 1 & & & & & & & & 1 & \\
\hline Dioxin & 1 & & & & & & & & 1 & \\
\hline Risk analysis & 0 & & & & & & & & & \\
\hline
\end{tabular}

*1 omitted from the objects of analysis

Table 8. Cross table of the keywords at the lecture on the precautionary principle and household waste management

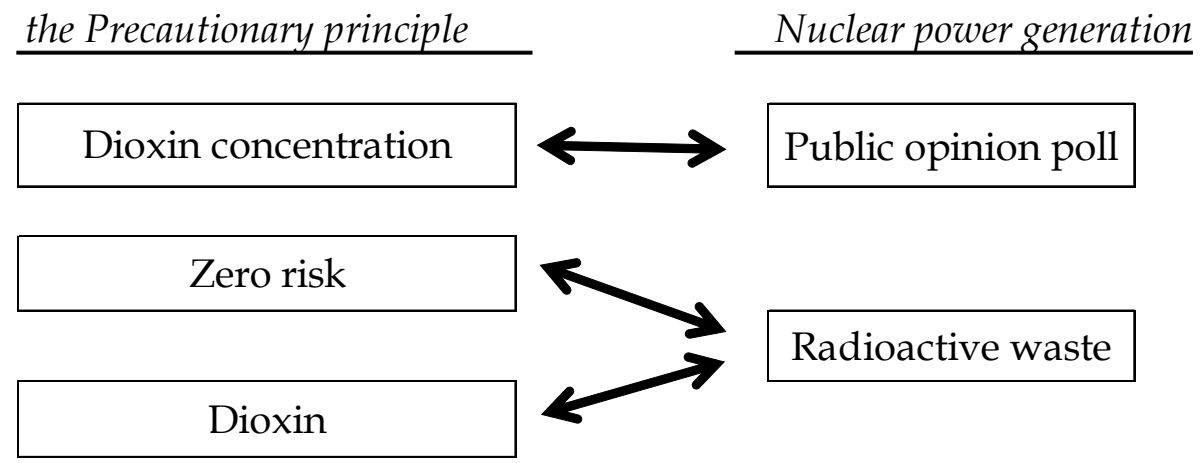

Fig. 8. Correspondence of the keyword (the Precautionary principle vs. nuclear power generation)

\section{Conclusion}

To find the communication point to promote the positive attitude toward the HLW disposal site, the author proposed the new approach of analyzing the consciousness of the students who stud rationally on the active position for constructive problem solution.

The previous analysis on the public risk communication had targeted on the majority of the subjects based on the assumption that the majority would represent the public so far. However, this new approach targeted on the minority of the subjects based on the 
assumption that a rational answer would not be made by the majority that was easy to make a heuristic decision but by the minority.

In this research, the author gave the lectures on the risk and desirable autonomous attitude in the some areas of environmental science. The students submitted the most impressive keyword after each lecture. The keywords were categorized by correspondence analysis and cluster analysis into two groups based on the assumed mental model. The two groups were characterized by the analysis of ANOVA on the result of questionnaire survey on nuclear power generation and high level radioactive waste disposal. One group consisted of the students who made a decision positively considering they were responsible for high level radioactive site selection. The other group consisted of the students who made a decision negatively considering they would not like to be involved in this issue.

The author paid attention to the former group and succeeded in deriving the common consciousness from the keywords of lectures on nuclear power generation, household waste management, and the precautionary principle.

It was observed from the keywords of nuclear power generation and household waste management, that economic efficiency, global relationship, sustainability and respect of individual opinion were common value among the active group, whose consciousness were positive towards HLW disposal site selection.

It was observed from the keywords of nuclear power generation and the precautionary principle, that antipathy for the seeing from up to down were common. Scientific or analytical viewpoints were also common among the active group.

In order to alleviate the reluctance for uncertainty of those who show resistance of being in the contiguity of HLW disposal facility, it should be significant for utilizing those common values interpreted along with this research for risk communication between citizen and governmental authorities. However, since this research has been mainly focusing on the area of risk communication between nuclear power generation and household waste management, and between nuclear power generation and the precautionary principle, there should be further researches conducted in the remaining areas such as global warming and other themes.

\section{Acknowledgment}

The author gratefully acknowledges the support of Tetsuya Kawamura, Prof. of Ochanomizu Women University, who gave the chance to lecture, and students of Ochanomizu Women University. The author gratefully acknowledges the advice from Hidekazu Yoshikawa, Prof. emeritus of Kyoto University.

\section{References}

Kugo, A., et al. (2005) Text Mining Analysis of Public Comments Regarding High-level Radioactive Waste Disposal, Journal of Nuclear Science and Technology, Vol. 42, No. 9, pp. 755-767, Atomic Energy Society of Japan.

Kugo, A., et al. (2008) Study on risk communication by using Web system for the social consensus towards HLW final disposal, International Review Journal of Progress in Nuclear Energy, Elsevier Ltd. Vol.50. pp. 700-708. 
Ohsumi, N. \& Levert, L. (2000) Analyzing Open-ended Questions: Some Experimental Results for Textual Data Analysis Based on InfoMiner, Proceedings of the Institute of Statistical Mathematics Vol.42. No.2, pp. 339-376.

Yoshikawa, H., et al. (2007) Human Interface for Symbiosis, Proceedings of the 2nd Conference of Trans-disciplinary Federation of Science and Technology, Kyoto, Japan. 